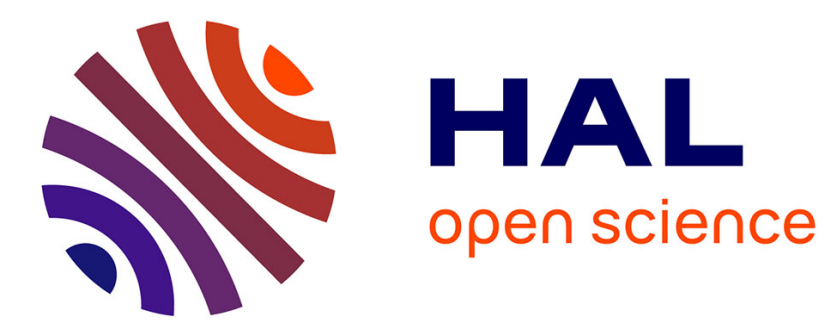

\title{
Physics of Substellar Objects: Interiors, Atmospheres, Evolution
}

\author{
Tristan Guillot
}

\section{To cite this version:}

Tristan Guillot. Physics of Substellar Objects: Interiors, Atmospheres, Evolution. Astrophysics [astroph]. Université Nice Sophia Antipolis, 2003. tel-00549887

\section{HAL Id: tel-00549887 \\ https://theses.hal.science/tel-00549887}

Submitted on 22 Dec 2010

HAL is a multi-disciplinary open access archive for the deposit and dissemination of scientific research documents, whether they are published or not. The documents may come from teaching and research institutions in France or abroad, or from public or private research centers.
L'archive ouverte pluridisciplinaire HAL, est destinée au dépôt et à la diffusion de documents scientifiques de niveau recherche, publiés ou non, émanant des établissements d'enseignement et de recherche français ou étrangers, des laboratoires publics ou privés. 


\section{Physique des Objets Substellaires \\ Intérieurs, Atmosphères, Evolution}

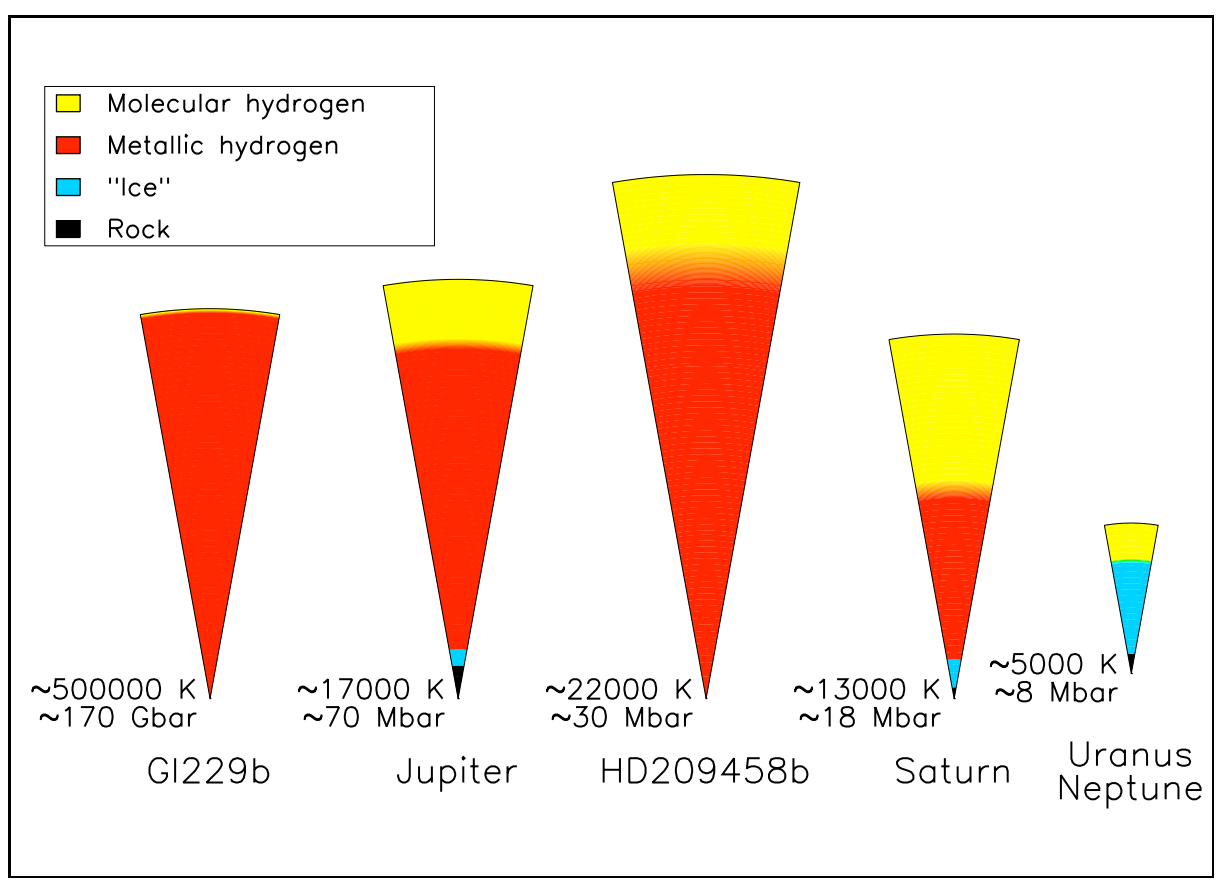

Présentée par

\section{Tristan Guillot}

Soutenue le 4 juin 2003 à l'Observatoire de la Côte d'Azur devant la commission d'examen :

Gilles Chabrier

Marianne Faurobert

Hal Levison

Michel Mayor

Pierre Morel
Rapporteur

Présidente

Rapporteur 
Toutes les étoiles visibles à

l'œil nu doivent leur brillance momentanée aux réactions nucléaires qui ont

lieu dans leur intérieur. Ceci en fait des joyaux

dans notre ciel nocturne, mais les amènera à une fin tragique, dans laquelle elles exploseront pour devenir soit des naines blanches dégénérées, des étoiles à neutrons ou des trous noirs. Une autre population, plus nombreuse, mais à peine visible, a choisi de vivre une vie morne mais tranquille et quasiment éternelle: ses individus font attention à ne pas devenir dépendant de l'hydrogène pour briller. Certains, dans leur jeunesse, consumment des substances moins énergétiques telles que le deutérium et

le lithium, mais épuisent rapidement leur stock. En conséquence, ils se refoidissent et se contractent progressivement, gardant intacts la plupart des éléments qui les ont formés.

Ces naines brunes et planètes géantes forment une nouvelle classe d'objets astronomiques. Ils comblent un fossé entre les étoiles et les planètes de notre Système Solaire. Leur étude nous informe sur nos origines, sur la formation des étoiles et des planètes. Elle nous aide aussi à comprendre et/ou tester des théories allant de la physique à haute pression, à la dynamique atmosphérique, en passant par

les effets de marées, la chimie, la formation de nuages...etc. Ce cours est focalisé sur quelques aspects physiques liés à l'étude théorique de ces objets substellaires: Je détaille leur évolution hydrostatique et sa modélisation, ce que nous savons de Jupiter, Saturne, Uranus et Neptune, de leur struture interne, comment les nuages façonnent leur apparence et contrôlent leur refroidissement, ce que nous pouvons apprendre des observations des naines brunes et planètes extrasolaires, et les conséquences des découvertes récentes sur notre vision de la formation planétaire. 


\section{Prélude}

Ce manuscrit est basé sur un cours préparé pour l'école de Saas-Fee 2001 (sous presse actuellement). Il est malheureusement en anglais, le travail de traduction qui avait été entrepris ayant, faute de temps, été abandonné après une trentaine de page. Mes propres travaux de recherches sont intimement mélés au cours. C'est le cas des publications suivantes (qui peuvent être aisément récupérées sur le web):

\section{Chapitre 4}

Guillot T., Gautier D., Chabrier G. \& Mosser B. 1994. Are the giant planets fully convective? Icarus 112, 337-353.

\section{Chapitre 5}

Guillot T., Chabrier G., Morel P. \& Gautier D. 1994. Non-adiabatic models of Jupiter and Saturn. Icarus 112, 354-367.

Guillot T. \& Morel P. 1995. CEPAM: a code for modeling the interiors of giant planets. Astron. E Astrophys. Suppl. 109, 109-123.

Guillot T., Gautier D. \& Hubbard W.B. 1997. New constraints on the composition of Jupiter from Galileo measurements and interior models. Icarus 130, 534-539.

Guillot T. 1999. A comparison of the interiors of Jupiter and Saturn. Plan. Space Sci. 47, 1183-1200.

Wuchterl G., Guillot T. \& Lissauer J.J. 2000. The formation of the giant planets. In Protostars 8 Planets IV (Eds. V. Mannings et al.), Univ. Arizona Press, pp.1081-1109.

Guillot T., Stevenson D.J., Hubbard W.B. \& Saumon D. 2003. The interior of Jupiter. In Jupiter (Eds. F. Bagenal et al.), Cambridge Univ. Press, sous presse.

\section{Chapitre 6}

Guillot T., Chabrier G., Gautier D, \& Morel P. 1995. Radiative transport and the evolution of Jupiter and Saturn. Astrophys. J. 450, 463-472.

Saumon D., Hubbard W.B., Burrows A., Guillot T., Lunine J.I. \& Chabrier G. 1996. A theory of extra-solar giant planets. Astrophys. J., 460, 993-1018.

Burrows A., Marley M.S., Hubbard W.B., Lunine J.I., Guillot T., Saumon D., Freedman R., Sudarsky D. \& Sharp C. 1997. A nongray theory of extrasolar giant planets and brown dwarfs. Astrophys. J. 491, 856-875.

Hubbard W.B., Guillot T., Marley M.S., Burrows A., Lunine J.I. \& Saumon D. 1999. Comparative evolution of Jupiter and Saturn. Plan. Space Sci. 47, 1175-1182.

\section{Chapitre 7}

Burrows A., Saumon D., Guillot T., Hubbard W.B. \& Lunine J.I. 1995. Prospects for detection of extra-solar giant planets by next-generation telescopes. Nature, 375, 299-301.

Guillot T. 1995. Condensation of methane, ammonia and water and the inhibition of convection in giant planets. Science 269, 1697-1699.

Marley M.S., Saumon D., Guillot T., Freedman R.S., Hubbard W.B., Burrows A. \& Lunine J.I. 1996. Atmospheric, evolutionary and spectral models of the brown dwarf Gliese 229 B. Science 272, 1919-1921.

Hueso R., Sanchez-Lavega A. \& Guillot T. 2002. A model for large-scale convective storms in Jupiter. JGR Planets, 107. 


\section{Chapitre 8}

Guillot T., Burrows A., Hubbard W.B., Lunine J.I., Saumon D. 1996. Giant planets at small orbital distances. Astrophys. J. Letters 459, L35-38.

Trilling D., Benz W., Guillot T., Lunine J.I., Hubbard W.B. \& Burrows A. 1998. Orbital evolution and migration of giant planets: modeling extrasolar planets. Astrophys. J. 500, 428-439.

Guillot T. 1999. Review: Interiors of giant planets inside and outside the solar system. Science 286, $72-77$.

Rauer H., Bockelée-Morvan D., Coustenis A., Guillot T. \& Schneider J. 2000. Search for an exosphere around 51 Peg B with ISO. Astron. Astrophys. 355, 573-580.

Burrows A., Guillot T., Hubbard W.B., Marley M.S., Saumon D., Lunine J.I. \& Sudarsky D. 2000. On the radii of close-in giant planets. Astrophys. J. Lett. 534, L97-L100.

Guillot T. \& Showman A.P. 2002. Evolution of "51 Peg b-like" planets. Astron. Astrophys. 385, $156-165$.

Showman A.P. \& Guillot T. 2002. Atmospheric circulation and tides of "51 Peg b-like" planets. Astron. Astrophys. 385, 166-180.

La page de garde illustre les structures internes respectives d'une naine brune (Gl229B), de trois

planètes géantes (Jupiter, Saturne et la "Pgaside" HD209458b) et de deux géantes de glace (Uranus et Neptune). (Merci à Bill Hubbard). 


\section{Physics of Substellar Objects Interiors, Atmospheres, Evolution}

All stars visible to the naked eye owe their momentary brightness to nuclear reactions occurring in their interior. While this certainly makes them jewels of the night skies, it will eventually lead them to a tragic end, in which they will explode to become either degenerate white dwarfs, neutron stars or black holes. Another, more numerous but barely visible population has chosen to lead a dull but quiet and almost eternal life: these are careful not to ever become dependent on hydrogen to shine. Some, in their youth, do burn less energetic substances as deuterium and lithium, but they rapidly get short of supply. As a consequence, they steadily cool and contract, retaining intact most of the elements that made them.

These brown dwarfs and giant planets form an entirely new class of astronomical objects. They fill a gap between stars and the planets of our Solar System. Their study informs us on our origins, the formation of stars and planets. It can also help us to understand or test theories from high pressure physics, to atmospheric dynamics, tides, condensation and cloud formation...etc.

The course focuses on some physical aspects related to the theoretical study of these substellar objects: I detail their hydrostatic evolution and how it is modeled, what we can learn from Jupiter, Saturn, Uranus and Neptune, how the atmospheres of brown dwarfs and giant planets are key to their appearance and cooling, what we can learn from the recent observations of brown dwarfs and extrasolar planets, and how this affects our view of planet formation. 


\section{Contents}

1 "Our" giant planets as a basis for the study of substellar objects 1

1.1 Origins: role of the giant planets for planet formation . . . . . . . . . . . . 1

1.2 Gravity field and global properties . . . . . . . . . . . . . . . . . 2

1.3 Magnetic fields . . . . . . . . . . . . . . . . . . . . . . 3

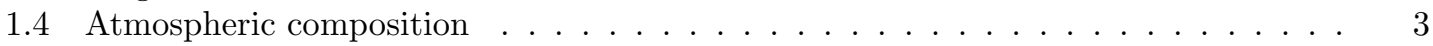

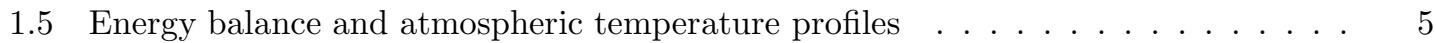

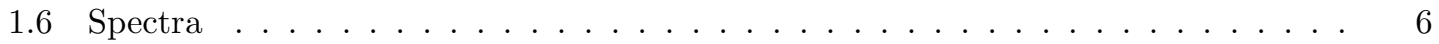

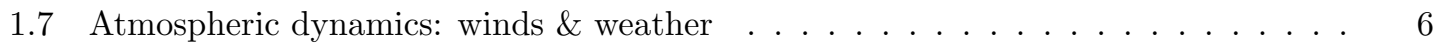

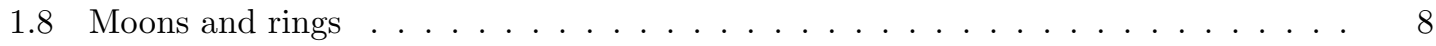

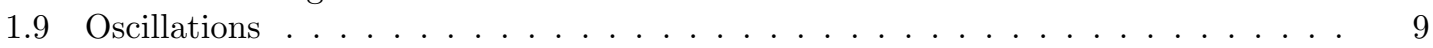

2 Basic equations, gravitational moments \& interior structures $\quad 11$

2.1 Hydrostatic equilibrium . . . . . . . . . . . . . . . . . . . . . 11

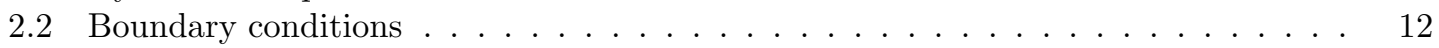

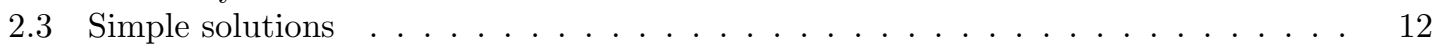

2.3 .1 Central pressure . . . . . . . . . . . . . . . . . . . . . . . 12

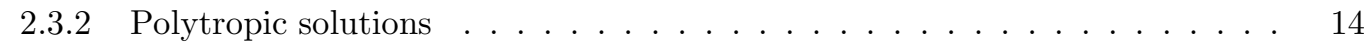

2.4 Mass-radius relation . . . . . . . . . . . . . . . . . . . 15

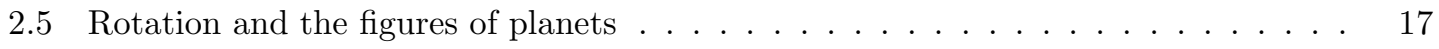

2.5.1 Hydrostatic equilibrium and symmetry breaking . . . . . . . . . . . 17

2.5.2 Equations for the level surfaces: principles . . . . . . . . . . . . . . . . . 19

2.5.3 The external potential: constraints from observations . . . . . . . . . 20

2.5.4 Effect of differential rotation . . . . . . . . . . . . . . . . . 21

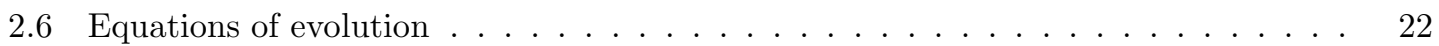

3 Equations of state $\quad 23$

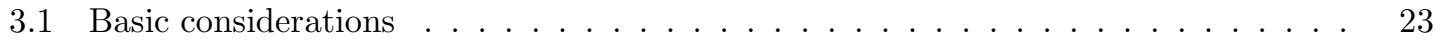

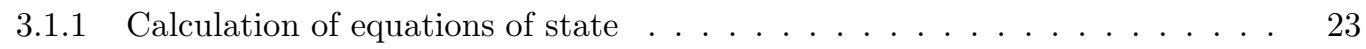

3.1 .2 The phase diagram . . . . . . . . . . . . . . . . . . 24

3.1 .3 The degenerate electron gas . . . . . . . . . . . . . . . . 26

3.1 .4 Pressure ionization . . . . . . . . . . . . . . . . . . . 27

3.2 Experiments and theoretical hydrogen EOSs . . . . . . . . . . . . . . . . 28

3.2.1 Reaching ultrahigh pressures: experimental results . . . . . . . . . . . 28

3.2 .2 Hydrogen: EOS calculations . . . . . . . . . . . . . . . . . . 30

3.3 Other elements . . . . . . . . . . . . . . . . . . . . . . . 32

3.3 .1 Approximate equations of state . . . . . . . . . . . . . 32 
3.3 .2 Miscibility of elements in hydrogen . . . . . . . . . . . . . . 34

4 Opacities \& heat transport $\quad 37$

4.1 Radiation absorption - basic considerations . . . . . . . . . . . . 37

4.2 Rosseland opacities . . . . . . . . . . . . . . . . . . . . . . . . 38

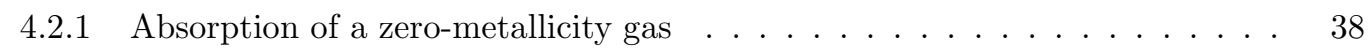

4.2 .2 Molecular line opacities . . . . . . . . . . . . . . . . . . . . . . . . . . . . . . . . . . . . . . . . . . .

4.2 .3 Line profiles . . . . . . . . . . . . . . . . . . . . 40

4.2 .4 Radiative Rosseland mean opacities . . . . . . . . . . . . . . . . 41

4.2 .5 Clouds and dust . . . . . . . . . . . . . . . . . . . . . 43

4.2 .6 Conductive opacities . . . . . . . . . . . . . . . . . . . 44

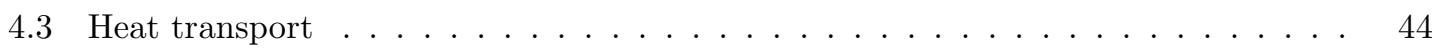

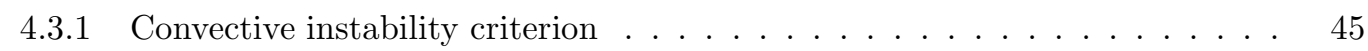

4.3 .2 Mixing length theory . . . . . . . . . . . . . . . . . 46

4.3.3 Properties of convection in substellar objects . . . . . . . . . . . . . . . 47

4.3 .4 Possible inhibitions of convection . . . . . . . . . . . . . . . 49

5 Interior structures of our giant planets: numerical integrations \& results $\quad 51$

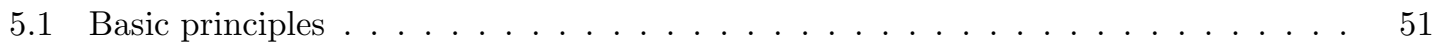

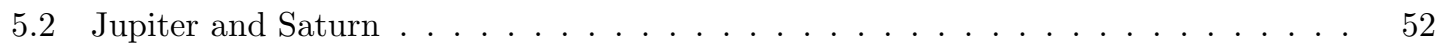

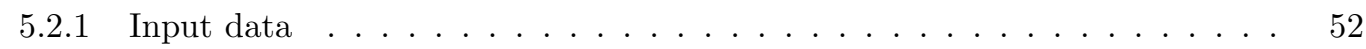

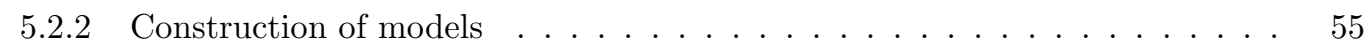

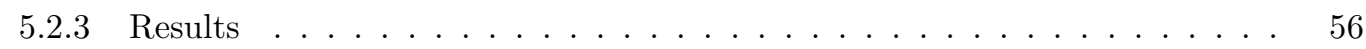

5.3 Uranus and Neptune . . . . . . . . . . . . . . . . . . . . . 59

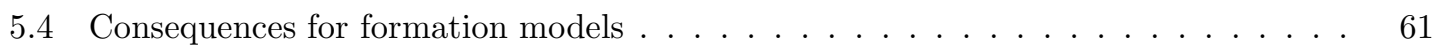

5.4 .1 The minimum mass solar nebula $\ldots \ldots \ldots \ldots$. . . . . . . . . 61

5.4 .2 Delivering planetesimals to the giant planets . . . . . . . . . . 62

5.4 .3 Possible formation scenarii . . . . . . . . . . . . . . . 63

6 Evolution of giant planets \& brown dwarfs $\quad 65$

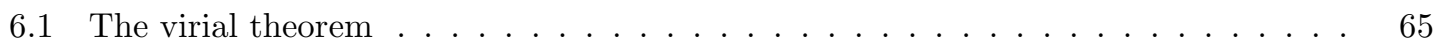

6.2 A semi-analytical model . . . . . . . . . . . . . . . . . . . 67

6.2 .1 Solution for isolated objects . . . . . . . . . . . . . . . . . . . . . . . . . . . . . 67

6.2 .2 Correction due to irradiation $\ldots \ldots \ldots \ldots \ldots \ldots$

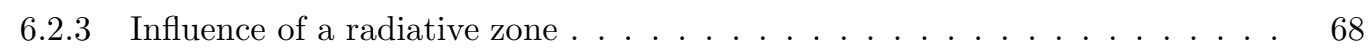

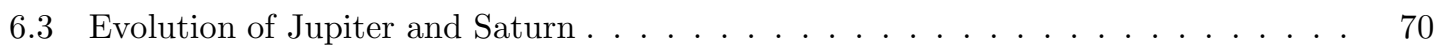

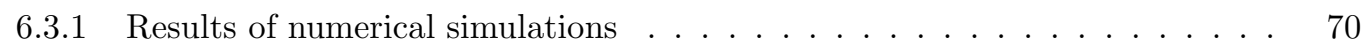

6.3.2 Including a hydrogen/helium phase separation . . . . . . . . . . . . 72

6.4 From giant planets to brown dwarfs . . . . . . . . . . . . . . . . . . 74

6.4.1 Nuclear reactions . . . . . . . . . . . . . . . . . . . . . . . 74

6.4.2 Brown dwarf models and results . . . . . . . . . . . . . . . . 75

6.4.3 Deuterium, lithium and hydrogen burning . . . . . . . . . . . . . . 80

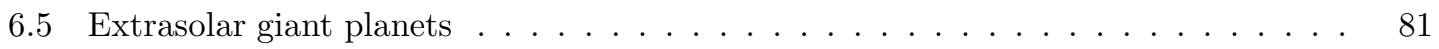


7 Spectra \& Atmospheres

7.1 Direct Observations of Substellar Objects . . . . . . . . . . . . . . . . . 83

7.1 .1 Gliese 229 B . . . . . . . . . . . . . . . . . . . 83

7.1.2 The new spectral classes . . . . . . . . . . . . . . . . . . 85

7.1.3 The colors of brown dwarfs . . . . . . . . . . . . . . . . . 85

7.1.4 Detection of very young substellar objects . . . . . . . . . . . . . . . 86

7.1.5 Rotation, magnetic activity and variability . . . . . . . . . . . . 88

7.2 Atmospheric models: importance of condensation . . . . . . . . . . . . . . . . 88

7.2.1 Basics of condensation . . . . . . . . . . . . . . . . . 89

7.2.2 Abundance of condensing species in an atmosphere . . . . . . . . . . . . . 90

7.2.3 Temperature profiles . . . . . . . . . . . . . . . . . . 92

7.2.4 Dust and clouds: relevant physical processes . . . . . . . . . . . . . . . 94

7.2.5 A transition from dusty to clear atmospheres . . . . . . . . . . . . 95

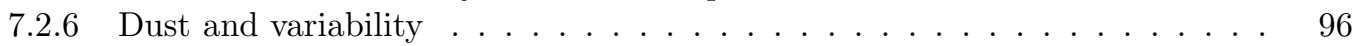

8 Pegasi Planets ("51 Peg b-like" Planets) 97

8.1 Introduction . . . . . . . . . . . . . . . . . . . . . . . . . . . . . . . . . . . . . . 97

8.2 Evolution of strongly irradiated giant planets . . . . . . . . . . . . . . . . . . . . . . . . . . . . . . . . . . . . . .

8.2.1 Including stellar heating: definitions . . . . . . . . . . . . . . . . . . . . . . . . . . . . . . . . . . 98

8.2.2 Temperature of irradiated atmospheres . . . . . . . . . . . . . . . . . . . 99

8.2.3 Hertzprung-Russell diagram . . . . . . . . . . . . . . . . . . 100

8.2.4 Growth of a radiative zone . . . . . . . . . . . . . . . . . . 102

8.2.5 Reproducing the radius of HD209458b . . . . . . . . . . . . . . . . 103

8.2.6 Evaporation/survival of Pegasi planets . . . . . . . . . . . . . . . . . . 104

8.3 Tidal effects . . . . . . . . . . . . . . . . . . . . . . . 105

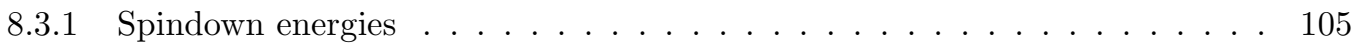

8.3.2 The equilibrium state . . . . . . . . . . . . . . . 106

8.4 Atmospheric dynamics . . . . . . . . . . . . . . . . . . . . . . . 108

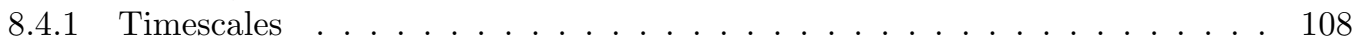

8.4.2 Possible circulation and atmospheric chemistry . . . . . . . . . . . . 109

8.4.3 Observational consequences . . . . . . . . . . . . . . 111 


\section{Chapter 1}

\section{"Our" giant planets as a basis for the study of substellar objects}

\subsection{Origins: role of the giant planets for planet formation}

The Solar System contains our Sun, which possesses more than $98 \%$ of the mass of the system, and eight planets orbiting around it in the same plane and same direction with quasi-circular orbits. The planets contain $99.5 \%$ of the angular momentum of the system. The four inner planets, Mercury, Venus, Earth and Mars have the highest densities, but more than $99.5 \%$ of the mass of the planetary system is in its four outer planets, Jupiter, Saturn, Uranus and Neptune. Most of the planets have moons, or natural satellites. Orbiting around the Sun, one also finds asteroids, Kuiper belt objects (including Pluto) and comets.

A picture emerges naturally from these observations: the formation of the planets in a circumstellar disk: the protosolar nebula. Planets formed close to the Sun naturally contain less volatiles and ices, while the outer planets were favored by the abundant presence of ices and could therefore grow fast enough to get hold of the surrounding hydrogen and helium of the nebula before its dissipation. In this picture, asteroids, Kuiper belt objects and comets all represent leftovers from an inefficient planet formation mechanism.

By their masses, the giant planets Jupiter, Saturn, Uranus and Neptune played a key role in this story. While the inner, terrestrial planets took tens of millions of years to reach their present masses, the giant planets had to form rapidly, before the gas of the protosolar nebula disappeared onto the star or was swept away from the system. They led to the ejection of numerous material, preventing the formation of a planet between Mars and Jupiter, and sending planetesimals into the Oort cloud, from where these remains of planetary formation come back once in a while as comets.

Their study therefore informs us on our origins. It also allows us to extend our knowledge beyond the frontiers of the Solar System and to model with confidence the other giant planets that have been found orbiting other stars. Before presenting the theoretical aspects of that understanding, I will detail here a few of the observations and measurements of significance for our purposes.

Most of the measurements at the basis of our understanding of the structure of our giant planets have been acquired by spacecraft missions: Pioneer 10 \& 11, Voyager 1 \& 2, Ulysses, Galileo, Cassini-Huygens. 


\subsection{Gravity field and global properties}

The mass of the giant planets can be obtained with great accuracy from the observation of the motions of their natural satellites: $317.834,95.161,14.538$ and 17.148 times the mass of the Earth $\left(1 \mathrm{M}_{\oplus}=5.97369 \times 10^{27} \mathrm{~g}\right)$ for Jupiter, Saturn, Uranus and Neptune, respectively. More precise measurements of their gravity field can be obtained through the analysis of the trajectories of spacecrafts during flyby, especially when they come close to the planet and preferably in a near-polar orbit. The gravitational field thus measured departs from a purely spherical function due to the planets' rapid rotation. The measurements are generally expressed by expanding the components of the gravity field on Legendre polynomials $P_{i}$ of progressively higher orders:

$$
V_{\text {ext }}(r, \theta)=-\frac{G M}{r}\left\{1-\sum_{i=1}^{\infty}\left(\frac{R_{\mathrm{eq}}}{r}\right)^{i} J_{i} P_{i}(\cos \theta)\right\},
$$

where $R_{\text {eq }}$ is the equatorial radius, and $J_{i}$ are the gravitational moments. Because the giant planets are very close to hydrostatic equilibrium the coefficients of even order are the only ones that are not negligible. We will see how these gravitational moments help us constrain the planets' interior density profiles.

Table 1.1: Characteristics of the gravity fields and radii

\begin{tabular}{lcccc}
\hline \hline & Jupiter & \multicolumn{1}{c}{ Saturn } & \multicolumn{1}{c}{ Uranus } & Neptune \\
\hline$M \times 10^{-29}[\mathrm{~g}]$ & $18.986112(15)^{\mathrm{a}}$ & $5.684640(30)^{\mathrm{b}}$ & $0.8683205(34)^{\mathrm{c}}$ & $1.0243542(31)^{\mathrm{d}}$ \\
$R_{\text {eq }} \times 10^{-9}[\mathrm{~cm}]$ & $7.1492(4)^{\mathrm{e}}$ & $6.0268(4)^{\mathrm{f}}$ & $2.5559(4)^{\mathrm{g}}$ & $2.4766(15)^{\mathrm{g}}$ \\
$R_{\text {pol }} \times 10^{-9}[\mathrm{~cm}]$ & $6.6854(10)^{\mathrm{e}}$ & $5.4364(10)^{\mathrm{f}}$ & $2.4973(20)^{\mathrm{g}}$ & $2.4342(30)^{\mathrm{g}}$ \\
$J_{2} \times 10^{2}$ & $1.4697(1)^{\mathrm{a}}$ & $1.6332(10)^{\mathrm{b}}$ & $0.35160(32)^{\mathrm{c}}$ & $0.3539(10)^{\mathrm{d}}$ \\
$J_{4} \times 10^{4}$ & $-5.84(5)^{\mathrm{a}}$ & $-9.19(40)^{\mathrm{b}}$ & $-0.354(41)^{\mathrm{c}}$ & $-0.28(22)^{\mathrm{d}}$ \\
$J_{6} \times 10^{4}$ & $0.31(20)^{\mathrm{a}}$ & $1.04(50)^{\mathrm{b}}$ & $\cdots$ & $\cdots$ \\
$P_{\omega} \times 10^{4}[\mathrm{~s}]$ & $3.57297(41)^{\mathrm{h}}$ & $3.83577(47)^{\mathrm{h}}$ & $6.2064^{\mathrm{i}}$ & $5.7996^{\mathrm{j}}$ \\
\hline \hline
\end{tabular}

The numbers in parentheses are the uncertainty in the last digits of the given value. The value of the gravitational constant used to calculate the masses of Jupiter and Saturn is $G=6.67259 \times 10^{-8}$ dyn.cm ${ }^{2} \cdot \mathrm{g}^{-1}$ (Cohen \& Taylor, 1987).

${ }^{a}$ Campbell \& Synott (1985)

b Campbell \& Anderson (1989)

c Anderson et al. (1987)

d Tyler et al. (1989)

${ }^{\mathrm{e}}$ Lindal et al. (1981)

${ }^{\mathrm{f}}$ Lindal et al. (1985)

${ }^{g}$ Lindal (1992)

${ }^{\mathrm{h}}$ Davies et al. (1986)

${ }^{\mathrm{i}}$ Warwick et al. (1986)

j Warwick et al. (1989)

Table 1.1 also indicates the radii obtained with the greatest accuracy by radio-occultation experiments. By convention, these radii and gravitational moments correspond to the 1 bar pressure level. The rotation periods show the relatively fast revolution of these planets: about 10 hours for Jupiter and Saturn, about 17 hours for Uranus and Neptune. The fact that this fast rotation visibly affects the figure (shape) of these planets is seen by the significant difference between the polar and equatorial radii. 
A first result obtained from the masses and radii indicated in Table 1.1 is the fact that these planets have low densities: $1.33,0.688,1.27$, and $1.64 \mathrm{~g} \mathrm{~cm}^{-3}$ for Jupiter, Saturn, Uranus and Neptune, respectively (these values are calculated using the planets' mean radii, as defined in section 2.5). Considering the compression that strongly increases with mass, one is led to a subclassification between the hydrogen-helium giant planets Jupiter and Saturn, and the "ice giants" Uranus and Neptune.

\subsection{Magnetic fields}

As the Earth, the Sun and Mercury, our four giant planets possess their own magnetic fields, as shown by the Voyager 2 measurements. The structures of these magnetic fields are very different from one planet to another and the dynamo mechanism that generates them is believed to be related to convection in their interior but is otherwise essentially unknown (see Stevenson 1983 for a review).

The magnetic field $\mathbf{B}$ is generally expressed in form of a development in spherical harmonics of the scalar potential $W$, such that $\mathbf{B}=-\nabla W$ :

$$
W=a \sum_{n=1}^{\infty}\left(\frac{a}{r}\right)^{n+1} \sum_{m=0}^{n}\left\{g_{n}^{m} \cos (m \phi)+h_{n}^{m} \sin (m \phi)\right\} P_{n}^{m}(\cos \theta) .
$$

$r$ is the distance to the planet's center, $a$ its radius, $\theta$ the colatitude, $\phi$ the longitude and $P_{n}^{m}$ the associated Legendre polynomials. The coefficients $g_{n}^{m}$ and $h_{n}^{m}$ are the magnetic moments that characterize the field. They are expressed in magnetic field units (i.e. the Gauss in c.g.s. units).

One can show that the first coefficients of relation (1.2) (for $n=0$ and $n=1$ ) correspond to the potential of a magnetic dipole such that $W=\mathbf{M} \cdot \mathbf{r} / r^{3}$ of moment:

$$
M=a^{3}\left\{\left(g_{1}^{0}\right)^{2}+\left(g_{1}^{1}\right)^{2}+\left(h_{1}^{1}\right)^{2}\right\}^{1 / 2} .
$$

Jupiter and Saturn have magnetic fields of essentially dipolar nature, of axis close to the rotation axis ( $g_{1}^{0}$ is much larger than the other harmonics); Uranus and Neptune have magnetic fields that are intrinsically much more complex. To provide an idea of the intensity of the magnetic fields, the value of the dipolar moments for the four planets are 4.27 Gauss $R_{\mathrm{J}}^{3}, 0.21$ Gauss $\mathrm{R}_{\mathrm{S}}^{3}, 0.23$ Gauss $\mathrm{R}_{\mathrm{U}}^{3}$, 0.133 Gauss $\mathrm{R}_{\mathrm{N}}^{3}$, respectively (Connerney et al. 1982; Acuña et al. 1983; Ness et al. 1986, 1989).

\subsection{Atmospheric composition}

The most important components of the atmospheres of our giant planets are also among the most difficult to detect: $\mathrm{H}_{2}$ and $\mathrm{He}$ have a zero dipolar moment. Also their rotational lines are either weak or broad. On the other hand, lines due to electronic transitions correspond to very high altitudes in the atmosphere, and bear little information on the structure of the deeper levels. The only robust result concerning the abundance of helium in a giant planet is by in situ measurement by the Galileo probe in the atmosphere of Jupiter (von Zahn et al. 1998). The helium mole fraction (i.e. number of helium atoms over the total number of species in a given volume) is $q_{\mathrm{He}}=0.1359 \pm 0.0027$. The helium mass mixing ratio $Y$ (i.e. mass of helium atoms over total mass) is constrained by its ratio over hydrogen, $X: Y /(X+Y)=0.238 \pm 0.05$. This ratio is by coincidence that found in the Sun's atmosphere, but because of helium sedimentation in the Sun's 
radiative zone, it was larger in the protosolar nebula: $Y_{\text {proto }}=0.275 \pm 0.01$ and $(X+Y)_{\text {proto }} \approx 0.98$. Less helium is therefore found in the atmosphere of Jupiter than inferred to be present when the planet formed. We will discuss the consequences of this measurement later: let us mention that the explanation invokes helium settling due to a phase separation in the interiors of massive and cold giant planets.

Helium is also found to be depleted compared to the protosolar value in Saturn's atmosphere. However, in this case the analysis is complicated by the fact that Voyager radio occultations apparently led to a wrong value. The current adopted value is now $Y=0.18-0.25$ (Conrath \& Gautier 2000), in agreement with values predicted by interior and evolution models (Guillot 1999; Hubbard et al. 1999). Finally, Uranus and Neptune are found to have near-protosolar helium mixing ratios, but with considerable uncertainty.

Table 1.2: Chemical species detected in the atmospheres of giant planets (courtesy of B. Bézard)

\begin{tabular}{|c|c|c|c|c|}
\hline & Jupiter & Saturn & $\overline{\text { Uranus }}$ & Neptune \\
\hline $\mathrm{H}_{2}$ & 0.864 & $0.86-0.90$ & $0.81-0.86$ & $0.77-0.82$ \\
\hline $\mathrm{He}$ & 0.134 & $0.10-0.14$ & $0.12-0.17$ & $0.16-0.22$ \\
\hline Rare Gases & $\mathrm{Ne}, \mathrm{Ar}, \mathrm{Kr}, \mathrm{Xe}$ & & & \\
\hline \multirow{4}{*}{$\begin{array}{l}\text { Species in } \\
\text { thermochemical } \\
\text { equilibrium }\end{array}$} & $\mathrm{CH}_{4}: 2 \times 10^{-3}$ & $\mathrm{CH}_{4}: 3-6 \times 10^{-3}$ & $\mathrm{CH}_{4}: \sim 2 \times 10^{-2}$ & $\mathrm{CH}_{4}: \sim 2 \times 10^{-2}$ \\
\hline & $\mathrm{NH}_{3}: 5 \times 10^{-3}$ & $\mathrm{NH}_{3}$ & & \\
\hline & $\mathrm{H}_{2} \mathrm{O}:>10^{-3}$ & $\mathrm{H}_{2} \mathrm{O}$ & & \\
\hline & $\mathrm{H}_{2} \mathrm{~S}: 8 \times 10^{-5}$ & & $\mathrm{H}_{2} \mathrm{~S} ?$ & $\mathrm{H}_{2} \mathrm{~S} ?$ \\
\hline Species in & $\mathrm{PH}_{3}$ & $\mathrm{PH}_{3}$ & & \multirow{4}{*}{$\mathrm{CO}$} \\
\hline \multirow{3}{*}{$\begin{array}{l}\text { thermochemical } \\
\text { disequilibrium }\end{array}$} & $\mathrm{CO}$ & $\mathrm{CO}$ & & \\
\hline & $\mathrm{GeH}_{4}$ & $\mathrm{GeH}_{4}$ & & \\
\hline & $\mathrm{AsH}_{3}$ & $\mathrm{AsH}_{3}$ & & \\
\hline $\begin{array}{l}\text { Photochemical } \\
\text { products }\end{array}$ & $\begin{array}{l}\mathrm{C}_{2} \mathrm{H}_{6}, \mathrm{C}_{2} \mathrm{H}_{2}, \\
\mathrm{C}_{2} \mathrm{H}_{4}, \mathrm{CH}_{3} \mathrm{C}_{2} \mathrm{H}, \\
\mathrm{C}_{6} \mathrm{H}_{6}\end{array}$ & $\begin{array}{l}\mathrm{C}_{2} \mathrm{H}_{6}, \mathrm{C}_{2} \mathrm{H}_{2} \\
\mathrm{CH}_{3} \mathrm{C}_{2} \mathrm{H}, \mathrm{C}_{4} \mathrm{H}_{2}, \\
\mathrm{C}_{6} \mathrm{H}_{6}, \mathrm{CH}_{3}\end{array}$ & $\mathrm{C}_{2} \mathrm{H}_{2}$ & $\begin{array}{l}\mathrm{C}_{2} \mathrm{H}_{6}, \mathrm{C}_{2} \mathrm{H}_{2}, \\
\mathrm{C}_{2} \mathrm{H}_{4}, \mathrm{CH}_{3}, \\
\mathrm{HCN}\end{array}$ \\
\hline Meteoritic flux & $\mathrm{H}_{2} \mathrm{O}, \mathrm{CO}$ & $\mathrm{H}_{2} \mathrm{O}$ & $\mathrm{H}_{2} \mathrm{O}$ & $\begin{array}{l}\mathrm{H}_{2} \mathrm{O} \\
\mathrm{CO}_{2}\left(\text { from } \mathrm{H}_{2} \mathrm{O}\right)\end{array}$ \\
\hline SL9 residuals & $\begin{array}{l}\mathrm{CO}, \mathrm{CO}_{2} \\
\mathrm{CS}, \mathrm{HCN}\end{array}$ & & & \\
\hline
\end{tabular}

The abundance of other elements (that I will call hereafter "heavy elements") bears crucial information for the understanding of the processes that led to the formation of these planets. Again, the most precise measurements are for Jupiter, thanks to the Galileo probe. Most of the heavy elements are enriched by a factor 2 to 4 compared to the solar abundance (Niemann et al. 1998; Owen et al. 1999). One exception is neon, but an explanation is its capture by the falling helium droplets (Roustlon \& Stevenson 1995). Another exception is water, but this molecule is affected by meteorological processes, and the probe was shown to have fallen into a dry region of Jupiter's atmosphere. There are strong indications that its abundance is at least solar. Possible very high interior abundances ( $\sim 10$ times the solar value) have also been suggested, either to explain waves propagation after the Shoemaker-Levy 9 impacts (Ingersoll et al. 1994) or as a scenario to explain the delivery of heavy elements to the planet (Gautier et al. 2001).

Assuming that all elements are enriched by a factor $\sim 3$ in Jupiter's interior, the total mass of heavy elements in the planet would be $\sim 18 \mathrm{M}_{\oplus}$. In the other planets, the case is considerably less clear as only the abundance of $\mathrm{CH}_{4}$ can be measured with confidence. As shown in Table 1.2 this ratio is consistent with an increased proportion of heavy elements when moving from Jupiter to Neptune. The problem of how these elements were delivered to these planets will be discussed later. 


\subsection{Energy balance and atmospheric temperature profiles}

Jupiter, Saturn and Neptune are observed to emit significantly more energy than they receive from the Sun (see Table 1.3). The case of Uranus is less clear. Its intrinsic heat flux $F_{\text {int }}$ is significantly smaller than that of the other giant planets. Detailed modeling of its atmosphere however indicate that $F_{\text {int }} \gtrsim 60 \mathrm{erg} \mathrm{cm}^{-2} \mathrm{~s}^{-1}$ (Marley \& McKay 1999). With this caveat, all four giant planets can be said to emit more energy than they receive from the Sun. Hubbard (1968) showed in the case of Jupiter that this can be explained simply by the progressive contraction and cooling of the planets.

Table 1.3: Energy balance as determined from Voyager IRIS data ${ }^{\mathrm{a}}$.

\begin{tabular}{lcccc}
\hline \hline & Jupiter & Saturn & Uranus & Neptune \\
\hline Absorbed power $\left[10^{23} \mathrm{erg} \cdot \mathrm{s}^{-1}\right]$ & $50.14 \pm 2.48$ & $11.14 \pm 0.50$ & $0.526 \pm 0.037$ & $0.204 \pm 0.019$ \\
Emitted power $\left[10^{23} \mathrm{erg} \cdot \mathrm{s}^{-1}\right]$ & $83.65 \pm 0.84$ & $19.77 \pm 0.32$ & $0.560 \pm 0.011$ & $0.534 \pm 0.029$ \\
Intrinsic power $\left[10^{23} \mathrm{erg} \cdot \mathrm{s}^{-1}\right]$ & $33.5 \pm 2.6$ & $8.63 \pm 0.60$ & $0.0344_{-0.034}^{+0.038}$ & $0.330 \pm 0.035$ \\
Intrinsic flux [erg.s $\left.{ }^{-1} . \mathrm{cm}^{-2}\right]$ & $5440 \pm 430$ & $2010 \pm 140$ & $42_{-42}^{+47}$ & $433 \pm 46$ \\
Bond albedo [] & $0.343 \pm 0.032$ & $0.342 \pm 0.030$ & $0.300 \pm 0.049$ & $0.290 \pm 0.067$ \\
Effective temperature [K] & $124.4 \pm 0.3$ & $95.0 \pm 0.4$ & $59.1 \pm 0.3$ & $59.3 \pm 0.8$ \\
1-bar temperature ${ }^{\mathrm{b}}[\mathrm{K}]$ & $165 \pm 5$ & $135 \pm 5$ & $76 \pm 2$ & $72 \pm 2$ \\
\hline \hline a After Pearl \& Conrath (1991) & & & & \\
b Lindal (1992) & & & &
\end{tabular}

A crucial consequence of the presence of an intrinsic heat flux is that it requires high internal temperatures $(\sim 10,000 \mathrm{~K}$ or more), and that consequently the giant planets are fluid (not solid) (Hubbard 1968; see also Hubbard et al. 1995). Another consequence is that they are essentially convective, and that their interior temperature profile are close to adiabats. We will come back to this in more details.

The deep atmospheres (more accurately tropospheres) of the four giant planets are indeed observed to be close to adiabats, a result first obtained by Trafton (1967), but verified by radiooccultation experiments by the Voyager spacecrafts, and by the in situ measurement from the Galileo probe (fig. 1.1). The temperature profiles show a temperature minimum, in a region near 0.2 barcalled the tropopause. At higher altitudes, in the stratosphere, the temperature gradient is negative (increasing with decreasing pressure). In the regions that we will be mostly concerned with, in the troposphere and in the deeper interior, the temperature always increases with depth. It can be noticed that the slope of the temperature profile in fig 1.1 becomes almost constant when the atmosphere becomes convective, at pressures of a few tens of bars, in the four giant planets.

It should be noted that the 1 bar temperatures listed in table 1.3 and the profiles shown in Fig. 1.1 are retrieved from radio-occultation measurements using a helium to hydrogen ratio which, at least in the case of Jupiter and Saturn, was shown to be incorrect. The new values of $Y$ are found to lead to increased temperatures by $\sim 5 \mathrm{~K}$ in Jupiter and $\sim 10 \mathrm{~K}$ in Saturn (see Guillot 1999). However, to make things simple (!), the Galileo probe found a 1 bar temperature of $166 \mathrm{~K}$ (Seiff et al. 1998), and generally a good agreement with the Voyager radio-occultation profile with the wrong $\mathrm{He} / \mathrm{H}_{2}$ value.

When studied at low spatial resolution, it is found that all four giant planets, in spite of their inhomogeneous appearances, have a rather uniform brightness temperature, with pole-to-equator latitudinal variations limited to a few kelvins (e.g. Ingersoll et al. 1995). However, in the case of Jupiter, some small regions are known to be very different from the average of the planet. This 


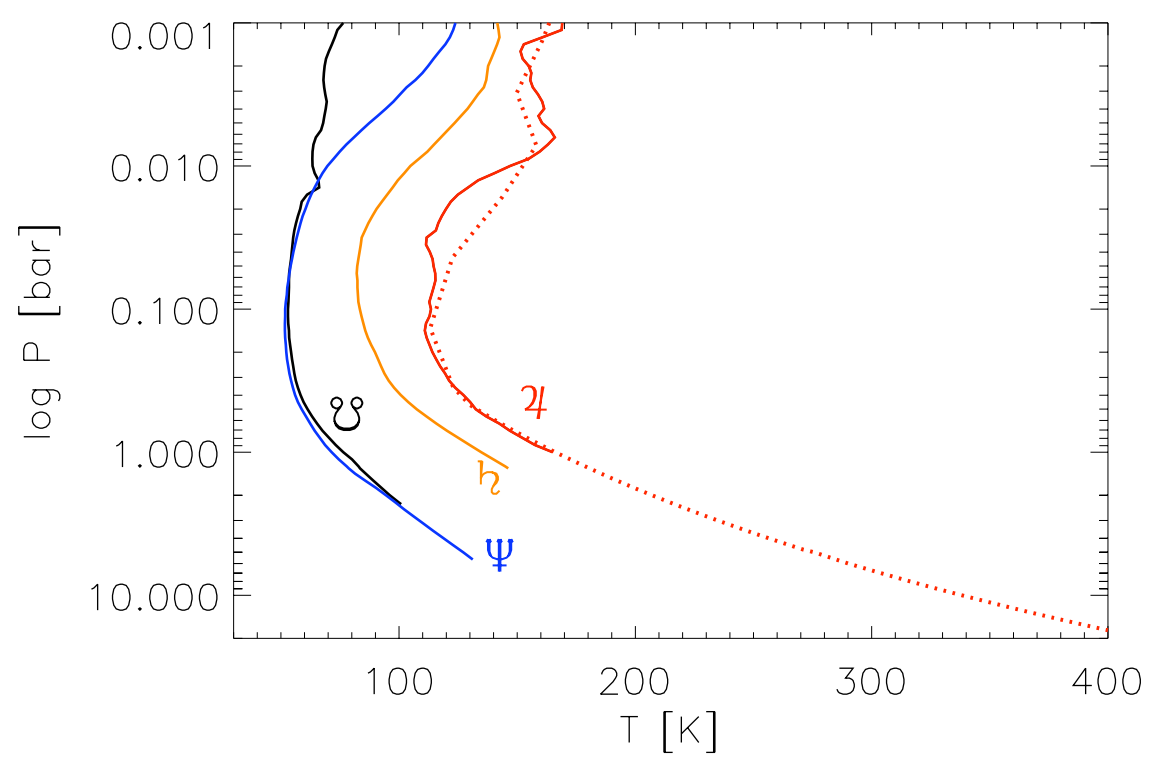

Figure 1.1: Atmospheric temperatures as a function of pressure for Jupiter, Saturn, Uranus and Neptune, as obtained from Voyager radio-occultation experiments (see Lindal 1992). The dotted line corresponds to the temperature profile retrieved by the Galileo probe, down to 22 barand a temperature of $428 \mathrm{~K}$ (Seiff et al. 1998).

is the case of hot spots, which cover about $1 \%$ of the surface of the planet at any given time, but contribute to most of the emitted flux at 5 microns, due to their dryness (absence of water vapor) and their temperature brightness which can, at this wavelength, peak to $260 \mathrm{~K}$. This fact is to be remembered when analyzing e.g. brown dwarfs spectra.

\subsection{Spectra}

A spectrum of a jovian hot spot obtained from the Galileo orbiter is shown in fig. 1.2. It demonstrates the complex structure of a planet, and the significant departures from a black-body radiation. At short wavelengths $(\lambda \lesssim 3 \mu \mathrm{m}$, the spectrum is dominated by the directly reflected solar light. At longer wavelengths, the thermal radiation dominates. The spectrum is dominated by the absorption bands of methane with some absorption by ammonia; water lines are seen around $5 \mu \mathrm{m}$, and a number of less abundant chemical species (e.g. phosphine) contribute to this spectrum.

\subsection{Atmospheric dynamics: winds \& weather}

The atmospheres of all giant planets are evidently complex and turbulent in nature. This can for example be seen from the mean zonal winds (inferred from cloud tracking), which are very rapidly varying functions of the latitude (see e.g. Ingersoll et al. 1995): while some of the regions 


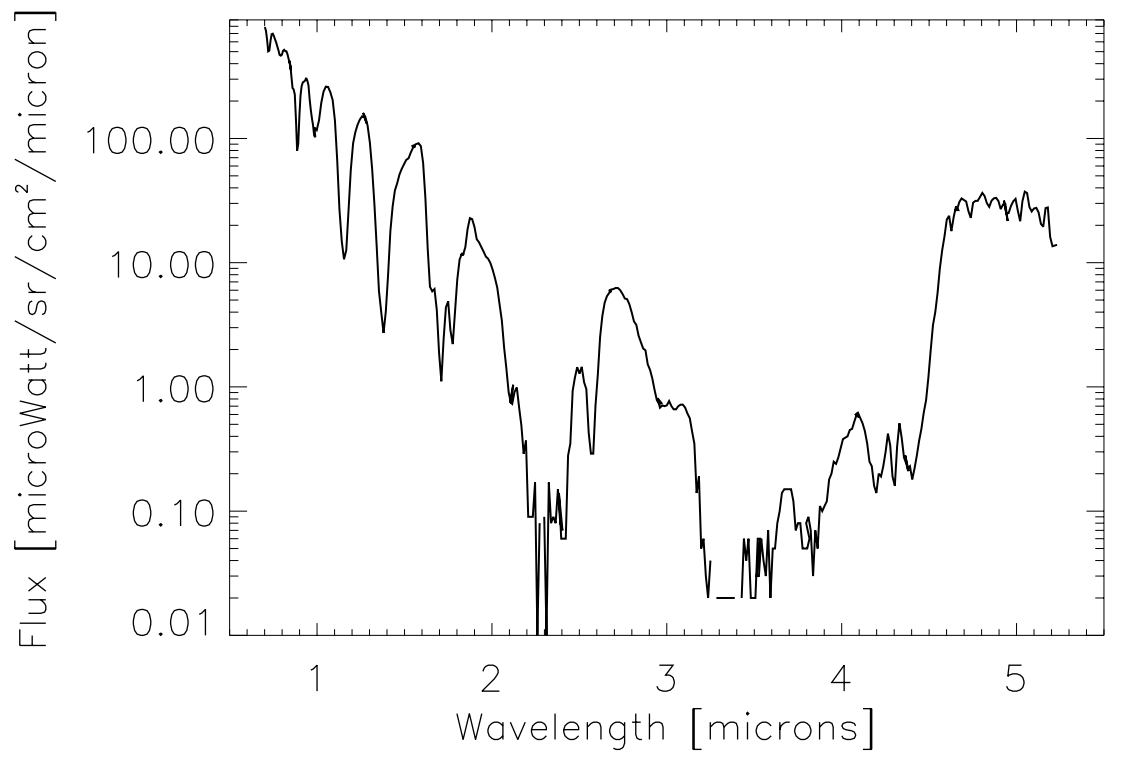

Figure 1.2: Flux emitted by a Jupiter hot spot as seen by the Galileo orbiter with NIMS. [From Carlson et al. 1996; Courtesy of P. Drossart].

rotate at the same speed as the interior magnetic fields ("system III"), most of the atmospheres do not. Jupiter and Saturn both have superrotating equators $\left(+100\right.$ and $+400 \mathrm{~m} \mathrm{~s}^{-1}$ in system III, for Jupiter and Saturn, respectively), Uranus and Neptune have subrotating equators, and superrotating high latitude jets. Neptune, which receives the smallest amount of energy from the Sun has the largest peak-to-peak latitudinal variations in wind velocity: about $600 \mathrm{~m} \mathrm{~s}^{-1}$. It can be noted that, contrary to the case of the strongly irradiated planets to be discussed later, the winds of Jupiter, Saturn, Uranus and Neptune, are significantly smaller than the surface speed due to the revolution of the planet on itself (from $12.2 \mathrm{~km} \mathrm{~s}^{-1}$ for Jupiter to $2.6 \mathrm{~km} \mathrm{~s}^{-1}$ for Neptune).

The observed surface winds are believed to be related to motions in the planets' interiors, which, according to the Taylor-Proudman theorem, should be confined by the rapid rotation to the plane perpendicular to the axis of rotation (e.g. Busse 1978). Unfortunately, no convincing model is yet capable of modeling with sufficient accuracy both the interior and the surface layers.

Our giant planets also exhibit planetary-scale to small-scale storms with very different temporal variations. For example, Jupiter's great red spot is a $12000 \mathrm{~km}$-diameter anticyclone found to have lasted for at least 300 years. Storms developing over the entire planet have even been observed on Saturn (Sanchez-Lavega et al. 1991). Neptune's storm system has been shown to have been significantly altered since the Voyager era. On Jupiter, small-scale storms related to cumulus-type cloud systems has been observed by Galileo, and lightning strikes can be monitored.

It is tempting to extrapolate these observations to the objects outside our Solar System as well. However, it is important to stress that an important component of the variability in the atmospheres of our giant planets is the presence of relatively abundant condensing chemical species: ammonia and water in the case of Jupiter and Saturn, and methane for Uranus and Neptune. These species 
can only condense (and thus provide the necessary latent heat) in very cold atmospheres. Other phenomena are however possible.

\subsection{Moons and rings}

A discussion of our giant planets motivated by the opportunity to extrapolate the results to objects outside our solar system would be incomplete without mentioning the moons and rings that these planets all possess. First, the satellites/moons can be distinguished from their orbital characteristics as regular or irregular. The first ones have generally circular, prograde orbits. The latter tend to have eccentric, extended, and/or retrograde orbits.

These satellites are numerous: After the Voyager era, Jupiter was known to possess 16 satellites, Saturn to have 18, Uranus 20 and Neptune 8. Recent extensive observation programs have seen the number of satellites increase considerably (see Gladman et al. 2001). At this date, $\sim 12$ have been detected around Jupiter, $\sim 12$ around Saturn, and 5 around Uranus. All of these new satellites are classified as irregular.

The presence of regular and irregular satellites is due in part to the history of planet formation. It is believed that the regular satellites have mostly been formed in the protoplanetary subnebulae that surrounded the giant planets (at least Jupiter and Saturn) at the time when they accreted their envelopes. On the other hand, the irregular satellites are thought to have been captured by the planet. This is for example believed to be the case of Neptune's largest moon, Triton, which has a retrograde orbit.

A few satellites stand out by having relatively large masses: it is the case of Jupiter's Io, Europa, Ganymede and Callisto, of Saturn's Titan, and of Neptune's Triton. Ganymede is the most massive of them, being about twice the mass of our Moon. However, compared to the mass of the central planet, these moons and satellites have very small weights: $10^{-4}$ and less for Jupiter, 1/4000 for Saturn, 1/25000 for Uranus and 1/4500 for Neptune. All these satellites orbit relatively closely to their giant planets. The furthest one, Callisto rotates around Jupiter in about 16 Earth days.

The four giant planets also have rings, whose material is probably constantly resupplied from their satellites. The ring of Saturn stands out as the only one directly visible with only binocular. In this particular case, its enormous area allows it to reflect a sizable fraction of the stellar flux arriving at Saturn, and makes this particular ring as bright as the planet itself. The occurrence of such rings would make the detection of extrasolar planets slightly easier, but it is yet unclear how frequent they can be, and how close to the stars rings can survive both the increased radiation and tidal forces. 


\subsection{Oscillations}

Last but not least, the case for the existence of free oscillations of the giant planets is still unresolved. Such a discovery would lead to great leaps in our knowledge of the interior of these planets, as can be seen from the level of accuracy reached by solar interior models since the discovery of its oscillations. Observations aimed at detecting modes of Jupiter have shown promising results (Schmider et al. 1991), but have thus far been limited by instrumental and windowing effects. A recent work by Mosser et al. (2000) puts an upper limit to the amplitude of the modes at $0.6 \mathrm{~m} \mathrm{~s}^{-1}$, and shows an increased energy of the Fourier spectrum in the expected range of frequencies. Observations from space of through an Earth-based network should be pursued in order to verify these results. 


\section{Chapter 2}

\section{Basic equations, gravitational moments \& interior structures}

\subsection{Hydrostatic equilibrium}

A very pleasing property of giant planets and brown dwarfs is that in spite of more than two decades of variation in mass, these objects basically obey the same physics: for most of their life, their interior is fluid and they are governed by the equilibrium between their internal pressure and their gravity. Unlike terrestrial planets, the characteristic viscosities are extremely small and can be neglected. The standard hydrostatic equation is thus:

$$
\frac{\partial P}{\partial r}=-\rho g
$$

where $P$ is the pressure, $\rho$ the density, and $g=G m / r^{2}$ the gravity ( $m$ is the mass, $r$ the radius and $G$ the gravitational constant).

Another equation is necessary to obtain the temperature as a function of pressure:

$$
\frac{\partial T}{\partial r}=\frac{\partial P}{\partial r} \frac{T}{P} \nabla_{T}
$$

While the equation itself is trivial, the calculation of the temperature gradient $\nabla_{T} \equiv(d \ln T / d \ln P)$ is not, and depends on the process by which the internal heat is transported. This term will be analyzed in a following section.

Thirdly, a special case of the mass conservation with zero velocity is:

$$
\frac{\partial M}{\partial r}=4 \pi r^{2} \rho
$$

Again, the physics of this equation is hidden in the dependency of the density $\rho$ with the pressure, temperature and composition, something given by the equation of state (see next section).

Finally, a crucial equation is derived from energy conservation considerations:

$$
\frac{\partial L}{\partial r}=4 \pi r^{2} \rho\left(\dot{\epsilon}-T \frac{\partial S}{\partial t}\right)
$$


where $L$ is the intrinsic luminosity, $t$ the time, $S$ the specific entropy (per unit mass), and $\dot{\epsilon}$ accounts for the sources of energy due e.g. to radioactivity or more importantly nuclear reactions. Generally it is a good approximation to assume $\dot{\epsilon} \sim 0$ for objects less massive than $\sim 13 \mathrm{M}_{\mathrm{J}}$, i.e. too cold to even burn deuterium (but we will see that in certain conditions this term may be useful, even for low mass planets).

\subsection{Boundary conditions}

At the center, $r=0 ; m=0, L=0$. The external boundary conditions are more complex to obtain because they depend on how energy is transported in the atmosphere. One possibility is to use the Eddington approximation, and to write (e.g. Chandrasekhar 1960):

$$
r=R: \quad \begin{aligned}
& T_{0}=T_{\text {eff }}, \\
& P_{0}=\frac{2}{3} \frac{g}{\kappa},
\end{aligned}
$$

where $\kappa$ is the opacity in $\mathrm{cm}^{2} \mathrm{~g}^{-1}$ (see section ). Note for example that in the case of Jupiter $T_{\text {eff }}=124 \mathrm{~K}, g=2600 \mathrm{~cm} \mathrm{~s}^{-2}$ and $\kappa \approx 5 \times 10^{-2}(P / 1 \mathrm{bar}) \mathrm{cm}^{2} \mathrm{~g}^{-1}$. This implies $P \approx 0.2 \mathrm{bar}$, which is actually close to Jupiter's tropopause, where $T \approx 110 \mathrm{~K}$.

Another possibility is to use an atmospheric model and to relate the temperature and pressure at a given level to the gravity and effective temperature of the object (or equivalently luminosity and radius):

$$
T_{0}=T_{0}\left(T_{\text {eff }}, g\right) ; \quad P_{0}=P_{0}\left(T_{\text {eff }}, g\right) .
$$

In the case of Jupiter and Saturn, an approximation often used is based on old calculations by Graboske et al. (1975). It takes the form

$$
T_{1 \mathrm{bar}}=K T_{\mathrm{eff}}^{a} g^{-b},
$$

where $K=1.5, a=1.243$ and $b=0.167$, all the quantities being expressed in cgs units. As shown by Fig. 2.1, this approximation is relatively good for effective temperatures lower than $200 \mathrm{~K}$, but it degrades substantially above that value (see also discussion in Saumon et al. 1996).

Note that these boundary conditions assume that the object is isolated. This is not the case of the giant planets of the solar system and for extrasolar planets for which the insolation can play an important role. We leave that problem for a further discussion.

\subsection{Simple solutions}

\subsubsection{Central pressure}

In order to estimate the central pressure, it is useful to write the hydrostatic equilibrium in a form which is independent on density:

$$
\frac{\partial P}{\partial m}=-\frac{G m}{4 \pi r^{4}} .
$$

Approximating by $m \approx M / 2, r \approx R / 2$ ( $M$ and $R$ being the total mass and radius, respectively) yields

$$
P_{\mathrm{c}} \approx \frac{2}{\pi} \frac{G M^{2}}{R^{4}}
$$




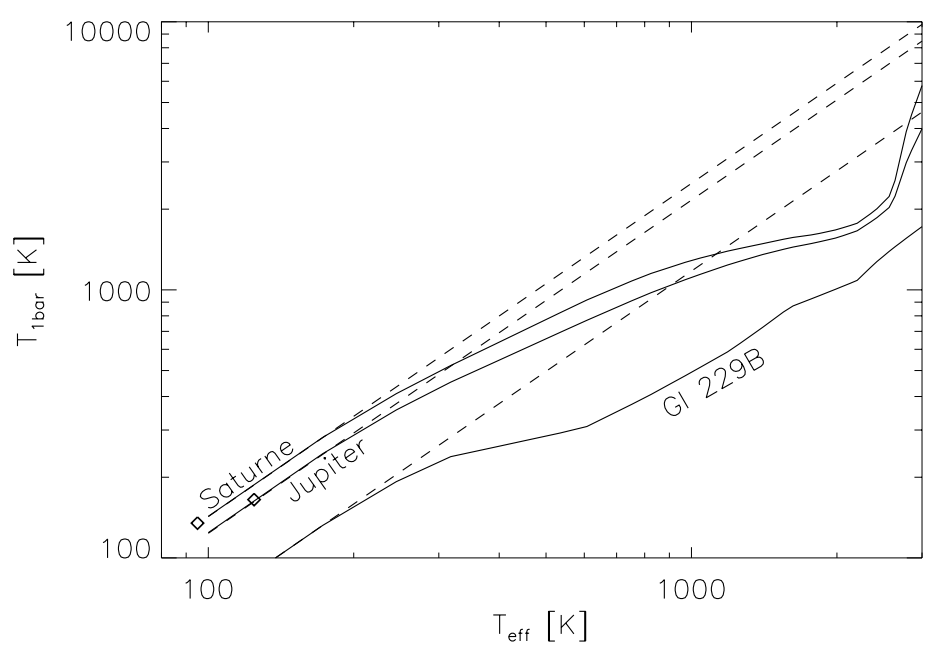

Figure 2.1: Comparison of the boundary condition obtained from Eq.(2.7) (dashed) to a grey atmosphere from Saumon et al. (1996) (plain), in the case of Saturn $\left(g \approx 1100 \mathrm{~cm} \mathrm{~s}^{-2}\right)$, Jupiter $\left(g \approx 2600 \mathrm{~cm} \mathrm{~s}^{-2}\right)$ and Gl229B $\left(g \approx 10^{5} \mathrm{~cm} \mathrm{~s}^{-2}\right)$.

Another simple solution is obtained by assuming uniform density $\bar{\rho}=3 M / 4 \pi R^{3}$. Equation 2.1 can then be integrated to obtain

$$
P_{\mathrm{c}} \approx \frac{3}{8 \pi} \frac{G M^{2}}{R^{4}}
$$

Knowing the mass and radius of a moon, planet or star, its central pressure can therefore be approximated within a factor of a few.

Using Eqs. $(2.9,2.10)$ the central pressure of the moon is found to be $17-91 \mathrm{kbar}, 1.7-9.1 \mathrm{Mbar}$ for the Earth, $12-64 \mathrm{Mbar}$ for Jupiter and 1.3-7.2 Gbar for the Sun. For comparison, the corresponding values given by more elaborate models are $\sim 40 \mathrm{kbar}, 3.6 \mathrm{Mbar}, 40$ to $70 \mathrm{Mbar}$ and 230 Gbar, respectively. The approximation is least successful in the case of the Sun, mostly because of the increase in density of the central regions $\left(\rho_{\mathrm{c}} \approx 150 \mathrm{~g} \mathrm{~cm}^{-3}\right)$.

When dealing with objects of small masses like planetary moons, the uniform density model is in fact a good approximation to the internal pressure, which can be shown to be:

$$
P(\xi) \approx \frac{4 \pi}{6} G R^{2} \bar{\rho}^{2}\left[1-\left(\frac{r}{R}\right)^{2}\right] .
$$

The central temperatures are more difficult to obtain a priori because contrary to main-sequence stars the interiors strongly depart from ideality. An a posteriori estimate uses the fact that these objects are mostly convective and that their temperature gradient $\nabla_{T} \equiv(d \ln T / d \ln P) \approx 0.3$. One then finds that $T \approx T_{\text {eff }}\left(P / P_{0}\right)^{\nabla_{T}}$, with $T_{\text {eff }}$ and $P_{0}$ being defined by the boundary conditions discussed in section 2.2. In the case of Jupiter, starting from $T(1 \mathrm{bar})=165 \mathrm{~K}$ and $P_{\mathrm{c}} \approx 12 \mathrm{Mbar}$, one gets $T_{\mathrm{c}} \approx 22000 \mathrm{~K}$, a relatively accurate estimate of the temperature at the bottom of the hydrogen-helium envelope. 


\subsubsection{Polytropic solutions}

A full integration of the set of differential equations is of course necessary to obtain the necessary precision on quantities such as pressure, temperature and density. However, it is sometime useful to use approximate analytical solutions to understand the underlying physics. One of these approximations, of considerable importance for stellar physics, is to assume a polytropic relation between pressure and density:

$$
P=K \rho^{1+1 / n},
$$

where $K$ is supposed constant, and $n$ is the polytropic index. Of course, this relation implicitly assumes that either density only depends on pressure not on temperature, or that the temperature profile is well-behaved and yields $K$ and $n$ constants.

This property is indeed verified in the limit when the pressure is due to non-relativistic fully degenerate electrons (e.g. Chandrasekhar 1939). In that case, a pure hydrogen plasma obeys the polytropic relation (2.12) with $n=3 / 2$ and a constant $K$ defined by fundamental physics (i.e. independent of $\left.M, T_{\mathrm{eff}} \ldots e t c.\right)$.

On the other hand, a perfect gas with a constant temperature gradient can be shown to obey a polytropic relation of index $n=1 /\left(1+1 / \nabla_{T}\right)$. In the case of a monoatomic perfect gas, $n=3 / 2$. It is important to notice that in that case $K$ is set by the atmospheric boundary condition: it depends on parameters such as the mass and effective temperature of the object considered.

A solution of the polytropic problem is obtained from the integration of the hydrostatic and Poisson equations:

$$
\left\{\begin{array}{l}
\frac{d P}{d r}=-\frac{d \Phi}{d r} \rho, \\
\frac{1}{r^{2}} \frac{d}{d r}\left(r^{2} \frac{d \Phi}{d r}\right)=4 \pi G \rho,
\end{array}\right.
$$

where $\Phi$ is the gravitational potential. The problem can be solved with some algebra. With the following change of variables,

$$
\begin{aligned}
z & =A r, \quad A^{2}=\frac{4 \pi G}{(n+1) K} \rho_{\mathrm{c}}^{\frac{n-1}{n}} \\
w & =\frac{\Phi}{\Phi_{\mathrm{c}}}=\left(\frac{\rho}{\rho_{\mathrm{c}}}\right)^{1 / n},
\end{aligned}
$$

where $\rho_{\mathrm{c}}$ and $\Phi_{\mathrm{c}}$ are the central density and gravitational potential, respectively, one is led to the famous Lane-Emden equation (see Chandrasekhar 1939, Kippenhahn \& Weigert 1991 for a demonstration):

$$
\frac{1}{z^{2}} \frac{d}{d z}\left(z^{2} \frac{d w}{d z}\right)+w^{n}=0 .
$$

This equation possesses analytical solutions for $\mathrm{n}=0,1$ and 5 . For our purpose, it is sufficient to say that the solutions are characterized by the surface condition: $z_{n}$ such that $w\left(z_{n}\right)=0$ and by the derivative of the function $w$ at that point: $(d w / d z)_{z_{n}}$. It can be shown that the total mass and surface radius of a polytrope are such that:

$$
\begin{aligned}
M & =4 \pi \rho_{\mathrm{c}} R^{3}\left(-\frac{1}{z} \frac{d w}{d z}\right)_{z=z_{n}}, \\
R & =z_{n}\left[\frac{1}{4 \pi G}(n+1) K\right]^{1 / 2} \rho_{\mathrm{c}}^{\frac{1-n}{2 n}} .
\end{aligned}
$$


If we assume that $K$ and $n$ are independent of the mass and surface conditions of the object considered, it is easy to show that the mass-radius relation is such that

$$
R \propto M^{\frac{1-n}{3-n}} .
$$

First, one can notice that the exponent diverges for $n=3$. In this case, the Lane-Emden equation has only one solution: this leads to the Chandrasekhar limit for the mass of white dwarfs. Second, for uncompressible materials, $n=0$ and we can verify that $R \propto M^{1 / 3}$. Third, objects whose internal pressure is dominated by non-relativistic degenerate electrons (this is formally valid only in the white dwarfs regime) are such that $n=3 / 2$ (see section 3.1.3) and $R \propto M^{-1 / 3}$.

\subsection{Mass-radius relation}

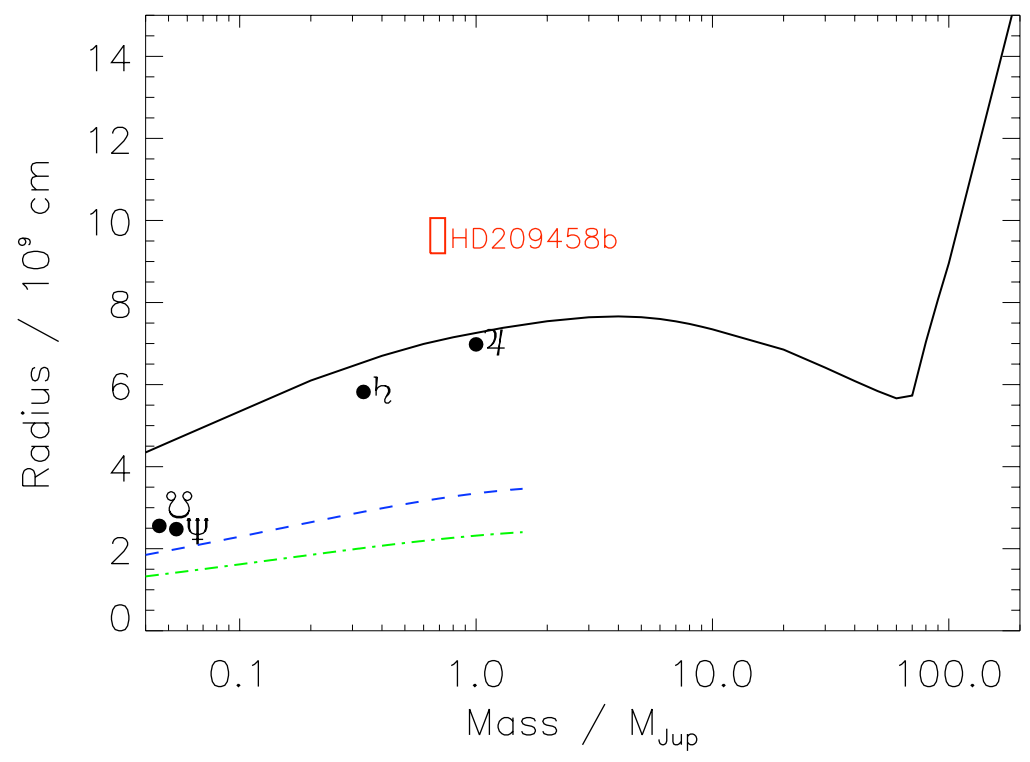

Figure 2.2: Radius versus mass for hydrogen-helium planets $(\mathrm{Y}=0.25)$ after $10 \mathrm{Ga}$ of evolution (plain line). An approximate mass-radius relation for zero-temperature water and olivine planets is shown as dashed and dash-dotted lines, respectively (Courtesy of W.B. Hubbard). The observed values for Uranus, Neptune, Saturn and Jupiter, as well as that for the Pegasi planet HD209458b are indicated.

The relation between mass and radius has very fundamental astrophysical applications. Most importantly is allows one to infer the gross composition of an object from a measurement of its mass and radius. This is especially relevant in the context of the discovery of extrasolar planets with both radial velocimetry and the transit method, as the two techniques yield relatively accurate determination of $M$ and $R$.

Figure 2.2 shows as a plain line the mass-radius relation of isolated hydrogen-helium objects (of approximate solar composition) after $10 \mathrm{Ga}$ of evolution. As could have been inferred from the 
polytropic solutions, this curve has a local maximum: at small masses, the compression is rather small so that the radius increases with mass (corresponding to a low polytropic index). (Note for example that in the case of the Earth, the central density is $\sim 13 \mathrm{~g} \mathrm{~cm}^{-3}$, to be compared with a mean density of $5.52 \mathrm{~g} \mathrm{~cm}^{-3}$ ). At large masses, degeneracy sets in and the radius decreases with mass (note from fig. 2.2 that it never quite reaches the white dwarf limit $R \propto M^{-1 / 3}$ ). At still larger masses (more than $70 \mathrm{M}_{\mathrm{J}}$ ), we get in the stellar regime, which is dominated by thermonuclear reactions, and thermal effects have to be taken into account.

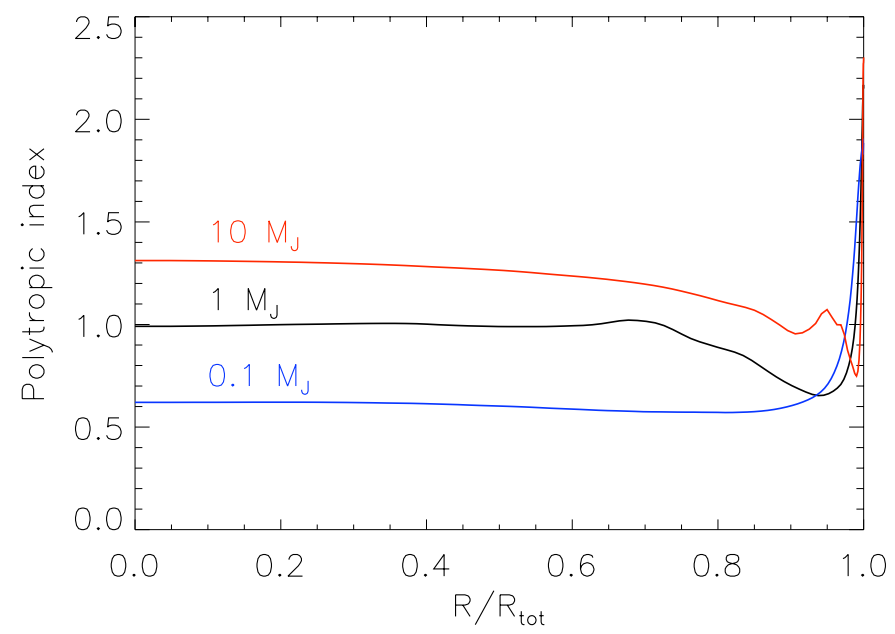

Figure 2.3: Polytropic index $n$ (such that $P \propto \rho^{1+1 / n}$ ) as a function of internal radius, for $0.1,1$ and $10 \mathrm{M}_{\mathrm{J}}$ isolated planets of solar composition after $10 \mathrm{Ga}$ of evolution.

The polytropic indexes of the isolated $0.1,1$ and $10 \mathrm{M}_{\mathrm{J}}$ are shown in Fig. 2.3. At small masses, $n$ is effectively rather small and the tends toward a uniform density solution. At around the mass of Jupiter, we get $n \sim 1$, which effectively corresponds to a maximum in the polytropic mass-radius relation (2.18). Above a mass of $\sim 4 \mathrm{M}_{\mathrm{J}}$, the radius starts decreasing with increasing mass, and effectively, the $10 \mathrm{M}_{\mathrm{J}}$ object has $n \approx 1.3$ in most of its interior. Equation (2.18) would imply $R \propto M^{-0.18}$, which is steeper than obtained on fig. 2.2. This is due to the fact that even after $10^{10}$ years, a $10 \mathrm{M}_{\mathrm{J}}$ object still retains part of its primordial heat and that $K$ can not be considered as independent of effective temperature and mass, as assumed in Eq. (2.18).

Another conclusion that can be derived from Fig. 2.2 is that the planets in our Solar System are not of solar composition: their radii lie below that predicted for $Y=0.25$ objects. Indeed, it can already be inferred that Jupiter, Saturn, and the two ice-giants Uranus and Neptune contain a growing proportion of heavy elements. The theoretical curves for olivine and ice planets predict even smaller radii however: even Uranus and Neptune contain 10 to $20 \%$ of their mass as hydrogen and helium.

An object is found above the hydrogen-helium curve: HD209458b. In this case, we will see that the planet has its evolution dominated by the intense stellar irradiation it receives. Thermal effects are no longer negligible: One cannot neglect the variations of the polytropic constant $K$ 
with mass. Instead of Eq. (2.18), one is led to:

$$
R \propto K^{\frac{n}{3-n}} M^{\frac{1-n}{3-n}} .
$$

The constant $K$ can be estimated through the surface boundary condition, assuming that the planetary interior is tied to the surface with an approximately constant polytropic index $n$ (a condition which is generally verified). Thus, using a perfect gas relation

$$
K=P_{0}^{-1 / n}\left(\frac{\mathcal{R} T_{0}}{\mu}\right)^{1+1 / n} .
$$

Let us assume that $T_{0}$ is, in the case of irradiated planets, set by the stellar insolation (and therefore independent of $M$ ). Using the Eddington boundary condition $P_{0} \propto g / \kappa$. The relation for the opacity $\kappa \propto P$ is generally valid for hot atmospheres not dominated by hydrogen-helium collision-induced absorption (see section 4). Therefore, a constant insolation and constant interior $n$ implies

$$
K \propto\left(\frac{M}{R^{2}}\right)^{-1 / 2 n} .
$$

It is then easy to show that the mass-radius relation for strongly irradiated planets becomes

$$
R \propto M^{\frac{1 / 2-n}{2-n}}
$$

Thus, for $n=3 / 2$, a relation valid for an adiabatic, ideal monoatomic gas, one finds $R \propto M^{-2}$. For $n=1$, one finds $R \propto M^{-1 / 2}$. Strongly irradiated hydrogen-helium planets of small masses are hence expected to have the largest radii. Note that this estimate implicitly assumes that $n$ is constant throughout the planet. The real situation is more complex because of the growth of a deep radiative region in most irradiated planets, and because of structural changes between the degenerate interior and the perfect gas atmosphere.

\subsection{Rotation and the figures of planets}

\subsubsection{Hydrostatic equilibrium and symmetry breaking}

We have thus seen that the knowledge of the mass and radius of a planet could inform us on its global composition. Fortunately, the giant planets in the Solar System are also fast rotators and their figure can also inform us more precisely on their internal composition. In the case of an

inviscid fluid rotating with an angular velocity $\boldsymbol{\Omega}(\mathbf{r})$, the hydrostatic equilibrium has to be written in the frame of rest of the system (see e.g. Pedlosky 1979):

$$
\frac{\nabla P}{\rho}=\nabla V-\boldsymbol{\Omega} \times(\boldsymbol{\Omega} \times \mathbf{r}),
$$

where the gravitational potential is defined as

$$
V(\mathbf{r})=G \int \frac{\rho\left(\mathbf{r}^{\prime}\right)}{\left|\mathbf{r}-\mathbf{r}^{\prime}\right|} d^{3} \mathbf{r}^{\prime}
$$


The resolution of eq. (2.23) is generally a complex problem. It can however be somewhat simplified by assuming that $|\boldsymbol{\Omega}| \equiv \omega$ is such that the centrifugal force can be derived from a potential:

$$
W(\mathbf{r})=\frac{1}{2} \omega^{2} r^{2} \sin ^{2} \theta,
$$

where $\theta$ is the angle from the rotation axis (colatitude). This implies that $\omega$ is either constant, or a function of the distance to the axis of rotation (rotation on cylinders).

The total potential is $U=V+W$ and the hydrostatic equilibrium can be written as

$$
\nabla P=\rho \nabla U .
$$

The figure of a fluid planet in hydrostatic equilibrium is then defined by the $U=$ cte level surface. The expression of $W$ shows that the centrifugal acceleration will be maximal at the equator. Since it tends to oppose gravity, it can be intuited that the planet's figure will depart from a sphere and become oblate, with a smaller polar radius than its equatorial radius. This was first demonstrated by Newton in 1687, but is in no way straightforward, and was contested by contemporaries, some advocating that the Earth's dimension should be larger at the poles!

Most of the problem lies in the breaking of the symmetry by rotation: the gravitational potential can no longer be integrated simply. We will summarize here one method, worked out by Lagrange, Clairaut, Darwin and Poincaré and detailed by Zarkhov \& Trubitsyn (1978). At its basis is a projection of the integrand of eq. (2.24) onto a basis of Legendre polynomials $P_{n}(\cos \psi)$ :

$$
\frac{1}{\left|\mathbf{r}-\mathbf{r}^{\prime}\right|}= \begin{cases}\frac{1}{r} \sum_{n=0}^{\infty}\left(\frac{r^{\prime}}{r}\right)^{n} P_{n}(\cos \psi) & \text { if } r \geq r^{\prime}, \\ \frac{1}{r} \sum_{n=0}^{\infty}\left(\frac{r^{\prime}}{r}\right)^{-n-1} P_{n}(\cos \psi) & \text { if } r<r^{\prime},\end{cases}
$$

where $\psi$ is the angle between the radius vectors $\mathbf{r}$ and $\mathbf{r}^{\prime}$. The Legendre polynomials are determined from the formula

$$
P_{n}(x)=\frac{1}{2^{n} n !} \frac{d^{n}}{d x^{n}}\left[\left(x^{2}-1\right)^{n}\right] .
$$

In particular, $P_{0}=1$ and $P_{2}(x)=\left(3 x^{2}-1\right) / 2$. These polynomials also have very important orthogonal properties that will not be detailed here.

Some geometry, the properties of Legendre polynomials and the assumption of hydrostatic equilibrium (azimuthal symmetry) allows one to write the gravitational potential in the form

$$
\begin{aligned}
V & =\frac{G}{r} \sum_{n=0}^{\infty}\left(r^{-2 n} D_{2 n}+r^{2 n+1} D_{2 n}^{\prime}\right) P_{2 n}(\cos \theta), \\
D_{2 n} & =\int_{r^{\prime} \leq r} \rho\left(r^{\prime}, \cos \theta^{\prime}\right) r^{2 n} P_{2 n}\left(\cos \theta^{\prime}\right) d^{3} \mathbf{r}^{\prime}, \\
D_{2 n}^{\prime} & =\int_{r^{\prime}>r} \rho\left(r^{\prime}, \cos \theta^{\prime}\right) r^{\prime-2 n-1} P_{2 n}\left(\cos \theta^{\prime}\right) d^{3} \mathbf{r}^{\prime} .
\end{aligned}
$$

The potential $V$ is thus projected on the basis of Legendre polynomials $P(\cos \theta)$. The $D_{2 n}$ and $D_{2 n}^{\prime}$ coefficients are complex functions. It is to be noted that this projection, as proposed by Lagrange poses a mathematical problem of divergence of the Legendre series between the sphere and level 
surface. Using a method initially proposed by Lyapunov, Trubitsyn showed that this expression is however valid because of the exact cancellation of the divergent terms (see Zharkov \& Trubitsyn 1978).

On the other hand, the centrifugal potential can be written on the same basis:

$$
W=\frac{1}{3} \omega^{2} r^{2}\left[1-P_{2}(\cos \theta)\right] .
$$

The total potential $U$ thus appears as a weighted sum (however complex) of Legendre polynomials.

\subsubsection{Equations for the level surfaces: principles}

The figure of a planet is determined by the level surfaces on which the total potential is constant. As shown by eq. (2.26), in hydrostatic equilibrium $\nabla P$ and $\nabla U$ are in the same direction. Taking the curl of that equation, one finds that $\nabla \rho \times \nabla U=0$. The surfaces of constant potential are also surfaces of constant pressure, density, and hence temperature. Hydrostatic equilibrium therefore also corresponds to barotropic equilibrium. (But remember our hypothesis that the centrifugal acceleration derives from a potential). These surfaces of constant $U$ are sought in the form:

$$
r(s, \cos \theta)=s\left[1+\sum_{n=0}^{\infty} s_{2 n}(s) P_{2 n}(\cos \theta)\right],
$$

where $s_{2 n}(s)$ are coefficients to be determined, and $s$ is chosen to be the radius of a sphere of equal volume (and hence, equal mass):

$$
\frac{4 \pi}{3} s^{3}=\frac{4 \pi}{3} \int_{0}^{1} r^{3}(s, \cos \theta) d \cos \theta .
$$

This allows one to integrate the angular part entering the calculation of the coefficients $D_{2 n}$ and $D_{2 n}^{\prime}$ in eq. (2.29). The solution of the problem is found by noticing that the total potential can now be written

$$
U(s, \cos \theta)=\frac{4 \pi}{3} G \bar{\rho} s^{2} \sum_{n=0}^{\infty} A_{2 n}(s) P_{2 n}(\cos \theta),
$$

where $\bar{\rho}$ is the planet's mean density. Since by definition the gravitational potential is constant on a level surface (fixed $s$ ), all coefficients $A_{2 n}(s)$ must be zero for $n \neq 0$. With eq. (2.32), we thus have $n+1$ equations for the $n+1$ variables $s_{0}, \ldots, s_{2 n}$. The problem can thus be solved for weak rotation rates $\omega$ by introducing a small parameter, $q$, the ratio of the centrifugal acceleration at the equator to the leading term in the gravitational acceleration:

$$
q=\frac{\omega^{2} R_{\mathrm{eq}}^{3}}{G M}
$$

$R_{\text {eq }}$ being the equatorial radius. One can show that $s_{0} \propto q$ and $s_{2 n} \propto q^{n}$ for $n \neq 0$. This system of integro-differential equations is rather complex and will not be given here (see Zharkov \& Trubitsyn 1978 for equations to third order).

With our choice of coordinates, the hydrostatic equation retains a simple form:

$$
\frac{\partial P}{\partial s}=\rho \frac{\partial U}{\partial s}
$$


i.e. the equation is now integrated with respect to the mean planetary radius. Furthermore, because of our assumption that the fluid remains barotropic, the other equations are unchanged. A detailed calculation of $U$ shows that

$$
\frac{1}{\rho} \frac{\partial P}{\partial s}=-\frac{G m}{s^{2}}+\frac{2}{3} \omega^{2} s+\frac{G M}{R^{3}} s \varphi_{\omega},
$$

where $\varphi_{\omega}$ is a slowly varying function of $s$ which, in the case of Jupiter varies from about $2 \times 10^{-3}$ at the center to $4 \times 10^{-3}$ at the surface.

\subsubsection{The external potential: constraints from observations}

As suggested previously, the effect of rotation is not only to complexify the equation for hydrostatic equilibrium. It also provide ones with the only way (yet) to probe the interiors of the giant planets of the solar system. This was first recognized by Sir H. Jeffreys (1923), but has seen significant progresses due to the flybys of the giant planets by the Pioneer and Voyager spacecrafts that allowed for a direct measurement of the planets' gravitational potentials.

The thus measured gravitational potentials are generally written in the form

$$
V_{\mathrm{ext}}(r, \cos \theta)=\frac{G M}{r}\left[1-\sum_{n=1}^{\infty}\left(\frac{a}{r}\right)^{2 n} J_{2 n} P_{2 n}(\cos \theta)\right],
$$

and the coefficients $J_{2 n}$ are the planet's gravitational moments. These are hence directly related to the coefficients $D_{2 n}$ defined by eq. (2.29), from which it can be shown that

$$
J_{2 n}=-\frac{1}{M a^{n}} D_{2 n} .
$$

(Note that because we are always outside the planet $r>r^{\prime}$ and the centrifugal potential does not appear since we are in an inertial coordinate system).

For example, the first gravitational moment can be calculated as

$$
\begin{aligned}
-M a^{2} J_{2} & =\int \rho\left(r^{\prime}\right) r^{2}\left(\frac{3}{2} \cos ^{2} \theta^{\prime}-\frac{1}{2}\right) d^{3} \mathbf{r}^{\prime} \\
& =\int \rho\left(r^{\prime}\right) \frac{1}{2}\left(2 r^{\prime 2} \cos ^{2} \theta^{\prime}-r^{\prime 2} \sin ^{2} \theta^{\prime}\right) d^{3} \mathbf{r}^{\prime} \\
& =\int \rho\left(r^{\prime}\right) \frac{1}{2}\left[\left(y^{2}+z^{2}\right)+\left(x^{2}+z^{2}\right)-2\left(x^{2}+y^{2}\right)\right] d^{3} \mathbf{r}^{\prime} \\
& =\frac{A+B-2 C}{2},
\end{aligned}
$$

where $A, B$ and $C$ are the principal moments of inertia of the planet with respect to axes $x, y$ and $z$, respectively.

The measured gravitational moments can thus be compared to the theoretically measured ones. For a planet in hydrostatic equilibrium, the odd moments $J_{2 n+1}$ are all zero while the even moments have a magnitude $J_{2 n} \propto q^{n}$. The high order gravitational moments also correspond to integrals with weighting functions peaking closer to the external layers of the planet. The information contained by the $\left\{J_{2 n}\right\}$ is therefore limited: without other information from e.g. global oscillations of the planet, it is impossible to accurately constrain the structure of the inner regions. 
Table 2.1: Parameters constraining interior structure

\begin{tabular}{llll}
\hline \hline & $q$ & $\Lambda_{2}$ & $C / M R_{\mathrm{eq}}^{2}$ \\
\hline Jupiter & 0.08923 & 0.165 & 0.26 \\
Saturn & 0.15491 & 0.105 & 0.22 \\
Uranus & 0.02951 & 0.119 & 0.23 \\
Neptune & 0.0261 & 0.136 & 0.24 \\
\hline \hline
\end{tabular}

Table 2.1 shows the values of the parameter $q$ and of the axial moment of inertia of the giant planets calculated from $J_{2}$ using the Radau-Darwin approximation (Zharkov \& Trubitsyn 1978):

$$
\frac{C}{M R_{\mathrm{eq}}^{2}} \approx \frac{2}{3}\left[1-\frac{2}{5}\left(\frac{5}{3 \Lambda_{2}+1}-1\right)^{1 / 2}\right],
$$

where we have introduced the linear response coefficient $\Lambda_{2} \equiv J_{2} / q$, and we have neglected second order terms proportional to the planets' flattening. Our four giant planets all have an axial moment of inertia substantially lower than the value for a sphere of uniform density, i.e. $2 / 5 M R^{2}$, indicating that they have dense central regions.

An analytical solution of the figure equation can be found for a polytropic equation of state of index $n=1\left(P \propto \rho^{2}\right)$, which is, as we have seen relevant for most of Jupiter's interior. In that case, one finds that (see Zharkov \& Trubitsyn 1978; Hubbard 1989), $\Lambda_{2}=0.173$ and thus $C / M R^{2}=0.263$, indeed very close to the value found for Jupiter. This shows already that Jupiter's core is small, relatively to the planet's total mass. It also indicates that Saturn, Uranus and Neptune have dense central regions and hence depart substantially from solar composition.

\subsubsection{Effect of differential rotation}

In order to be able to integrate the system of integro-differential equations, we have implicitly assumed a solid body rotation. The atmospheres of all giant planets is seen to rotate with a speed which is latitudinally dependent. These latitudinal variations amount to about $1 \%$ for Jupiter to more than $15 \%$ in the case of Neptune, from peak to peak.

A first consequence is that the gravitational potential calculated assuming solid body rotation will be different than if the interior rotation is, say, on cylinders. For a given structure, differential rotation such as imposed by the surface winds of Jupiter and Saturn increases the absolute values of the planets' gravitational moments. In order to account for that effect using solid body rotation, one has to use effective gravitational moments that are smaller in absolute value than those directly measured (Hubbard 1982).

Another interesting consequence concerns the high order gravitational moments, $J_{10}$ and above. Hubbard (1999) has shown that if the observed atmospheric rotation pattern persists deep enough into the interior (say to within a few $\%$ of the total radius beneath the atmospheric layer), then the gravitational moments will stop decreasing and reach a plateau at a value $\left|J_{n}\right| \approx 10^{-8}$ with $n \gtrsim 10$. This lends support to space missions that would enable a detailed mapping of the gravitational fields of the giant planets. This would require both a polar-like orbit and one (or better several) very close flybys. 


\subsection{Equations of evolution}

We have so far expressed the differential equations in terms of the radius $r$. This Eulerian approach has the inconvenience that the spatial variable can be a rapidly varying function of time (when, during the evolution, the contraction is fast). It is therefore generally more convenient to use a Lagrangian approach, in which the new independent coordinates are the mass $m$ and time $t$. This has the advantage that except in the case of mass loss/gain, the outer boundary condition is defined at a fixed $m=M$, the total mass of the object. Note that because of our definition of the radius as the mean radius, the effect of rotation is just to add two terms to the hydrostatic equation. Hereafter, we will use $r$ instead of $s$ as the mean radius (see e.g. Guillot \& Morel 1995 for a possible method to numerically resolve the equations). The system of differential equations becomes:

$$
\left\{\begin{aligned}
\frac{\partial P}{\partial m} & =-\frac{G m}{4 \pi r^{4}}+\frac{\omega^{2}}{6 \pi r}+\frac{G M}{4 \pi R^{3} r} \varphi_{\omega}, \\
\frac{\partial T}{\partial m} & =\left(\frac{\partial P}{\partial m}\right) \frac{T}{P} \nabla_{T}, \\
\frac{\partial r}{\partial m} & =\frac{1}{4 \pi r^{2} \rho}, \\
\frac{\partial L}{\partial m} & =\dot{\epsilon}-T \frac{\partial S}{\partial t},
\end{aligned}\right.
$$

The boundary conditions are as discussed in section 2.2, except that the variable is now $m$ instead of $r$. Note however that when studying the present-day interiors of Jupiter, Saturn, Uranus or Neptune, the most logical surface boundary condition is at a fixed temperature $T=T_{\text {surf }}$ and pressure $P_{\text {surf }}$, for $m=M$. Note that in that case, there is no time dependency, and the energy conservation equation cannot be integrated. This requires a priori setting the luminosity (usually by assuming that it is uniformly equal to the measured intrinsic luminosity of the planet). In all other cases, i.e. when considering the evolution of substellar objects, the outer boundary condition must depend on $L$ and $R$.

Most of the important physics in the system of equations (2.41) is hidden in several quantities: $\varphi_{\omega}$ contains the physics related to rotation discussed previously, but is generally a small perturbation. The term $\nabla_{T}$ depends on the process which transports the energy inside the planet and will be discussed in section 4 The density $\rho$ and specific entropy $S$ are functions of the temperature, pressure and composition. They have to be calculated independently using an appropriate equation of state, the subject of the next section. Finally, $\dot{\epsilon}$ accounts for any source of energy, e.g. thermonuclear reactions, radioactivity or heat dissipation. This term is generally neglected, but will be discussed for brown dwarfs, and also in the case of Pegasi planets. 


\section{Chapter 3}

\section{Equations of state}

\subsection{Basic considerations}

\subsubsection{Calculation of equations of state}

The knowledge of appropriate equations of state is at the basis of any modeling of substellar objects. Basically, for a given atomic composition, and two macroscopic thermodynamic variables, say temperature and volume, an equation of state is to provide all the other thermodynamic variables and their derivatives (pressure, internal energy, entropy, specific heat...etc.). As discussed by Fontaine, Graboske \& Van Horn (1977), the thermodynamic constraints that have to be satisfied for any equilibrium thermodynamic description of a single-phase material are:

$$
\begin{array}{ll}
\text { I. Accuracy } & P^{\operatorname{approx}}(T, V)=P^{\text {exact }}(T, V) . \\
\text { II. Stability } & U^{\text {approx }}(T, V)=U^{\text {exact }}(T, V) . \\
\text { III. Consistency } & \left(\frac{\partial P}{\partial V}\right)_{T}<0, \quad\left(\frac{\partial P}{\partial T}\right)_{V}=\left(\frac{\partial S}{\partial V}\right)_{T}=0 . \\
\text { IV. "Normality" } & \left(P+\left(\frac{\partial P}{\partial T}\right)_{V}>0, \quad\left(\frac{\partial^{2} P}{\partial V^{2}}\right)_{T}\right) .
\end{array}
$$

As noted by the authors, condition II is generally trivial to achieve; condition III is straightforward but often grossly violated; condition IV is not thermodynamically demanded, but holds for most $\rho, T$ values. Indeed, we will see one possible equation of state for which condition IV is violated.

The calculation of equations of state itself can become extremely complex. For our purposes, it will suffice to say that it can be split into two main groups: the "chemical" and "physical" pictures. In the chemical picture, one assumes that bound configurations (e.g. atoms, molecules) retain a definite identity and interact through pair potentials. The system of particles of species $\alpha$ confined to a volume $\mathrm{V}$ at temperature $\mathrm{T}$ is conveniently described by the Helmoltz free energy $F$, which is itself obtained from microscopic physics through

$$
F\left(\left\{N_{\alpha}\right\}, V, T\right)=-k T \ln Z\left(\left\{N_{\alpha}\right\}, V, T\right),
$$

where $N_{\alpha}$ denotes the number of particles and $Z$ is the canonical partition function of the system. Other thermodynamical quantities are then obtained from derivatives of $F$. For example,

$$
P=-\left(\frac{\partial F}{\partial V}\right)_{\left\{N_{\alpha}\right\}, T} .
$$


When confronted to ionization and/or dissociation, the actual composition of the system (i.e. abundances of electrons, ions, atoms and molecules) is obtained through a minimization of the free energy of the system. As discussed by Fontaine et al., the calculation of the free energy requires several assumptions that necessarily limit its accuracy. Its main drawback is the apriori definition of certain classes of particles, i.e. ions, atoms and molecules which necessitates the use of effective interaction potentials. The calculation can thus fail in states where more complex systems are formed and the distinction between bound and free states is not easily made.

Another method consists in directly computing the n-body Schrödinger equation of the quantumstatistical system. This approach is generally exact in the limit set by the computationally intensive method that has to be used to solve the problem. Within this physical picture, two main approaches have been used: restricted path integral Monte Carlo simulations, and density functional theory molecular dynamics. The first approach consists in solving the full problem for a limited number of protons and electrons in a box (64 of each at the most, with today's computers). The second approach involves local solutions to the problem and fails when both short range and long range interactions have to be taken into account.

\subsubsection{The phase diagram}

In terms of pressures and temperatures, the interiors of giant planets and brown dwarfs lie in a region for which accurate equations of state are extremely difficult to calculate. Some of the important phenomena that occur in these objects are illustrated by the phase diagram of hydrogen (figure 3.1).

The photospheres of these objects is generally relatively cold and at low pressure, so that hydrogen is in molecular form and the perfect gas conditions apply:

$$
P=\frac{\rho \mathcal{R} T}{\mu} ; \quad U=C_{V} T
$$

with $\mu \approx 2$ (neglecting helium atoms and heavy elements) and $C_{V} \approx 5 / 2 k$, due to the vibration of the hydrogen molecule.

As one goes deeper into the interior however, the molecules become closer to one another. The system progressively becomes a liquid, in which the interactions between molecules play an important role. This occurs when the intermolecular distance becomes of the same order as the size of a hydrogen molecule. Using real equations of state, it can be estimated that the perfect gas relation tends to underestimate the pressure by $10 \%$ or more when the density becomes larger than about $0.02 \mathrm{~g} \mathrm{~cm}^{-3}$ (pressures above $1 \mathrm{kbar}$ in the case of Jupiter).

At higher densities (or pressures) and relatively low temperatures, the electrons can become degenerate: in that limit, their momentum is not determined by the temperature of the mixture, but by the fact that, as fermions of spin $-1 / 2$ or $+1 / 2$, only two of them can be stacked in a cell $\Delta p \Delta V=h^{3}$. (Pauli's exclusion principle). The significance of this phenomenon can be measured through a degeneracy temperature parameter

$$
\theta=\frac{T}{T_{\mathrm{F}}}=\frac{2 m_{\mathrm{e}} k}{h^{2}}\left(\frac{8 \pi}{3} \mu_{\mathrm{e}} m_{\mathrm{u}}\right)^{2 / 3} \frac{T}{\rho^{2 / 3}},
$$

where $T_{\mathrm{F}}$ is the Fermi temperature, and the number density of electrons is $n_{\mathrm{e}}=\rho / \mu_{\mathrm{e}} m_{\mathrm{u}}$. The quantity $\mu_{\mathrm{e}}$ is the mean molecular weight per electron $\left(\mu_{\mathrm{e}} \approx 2 /(1+X)\right)$. The parameter $\theta$ can be defined regardless of the presence of bound states. However, in the presence of atoms and 


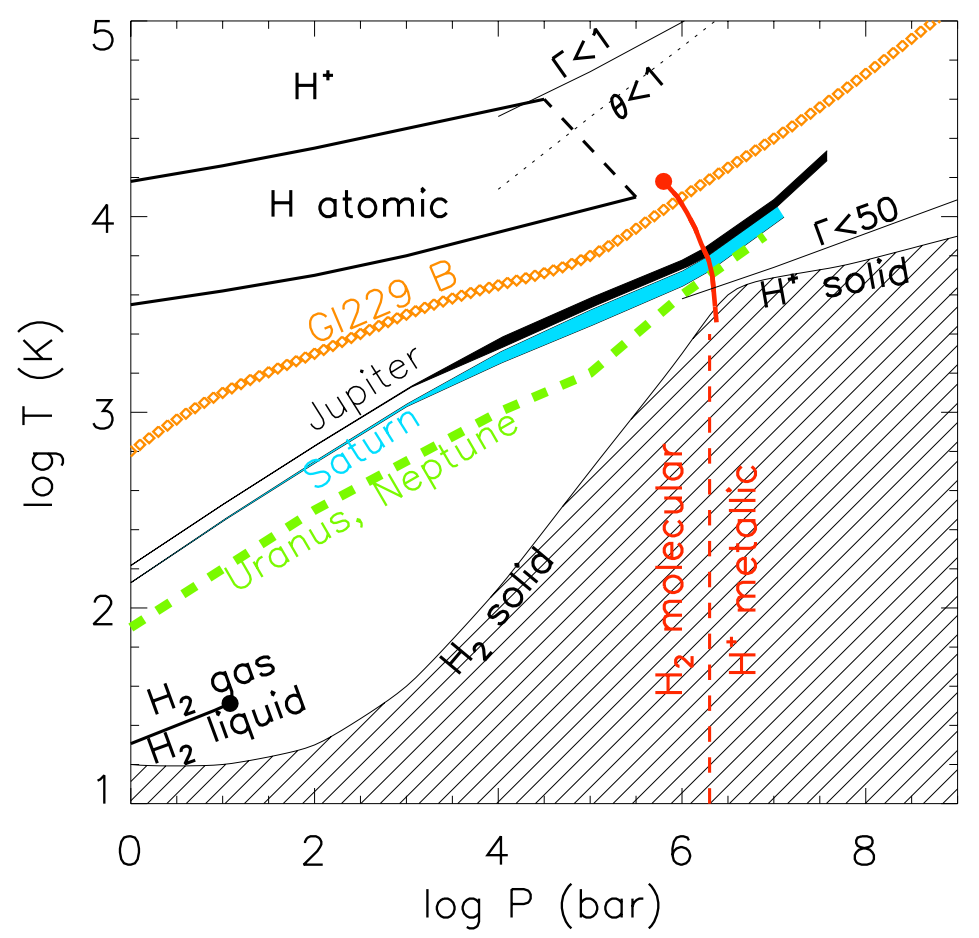

Figure 3.1: Phase diagram for hydrogen with the main phase transitions occurring in the fluid or gas phase. The temperature-pressure profiles for Jupiter, Saturn, Uranus, Neptune, and Gl229B (assumed to be a $30 \mathrm{M}_{\mathrm{J}}$ brown dwarf) are shown. The plain, almost vertical line near $1 \mathrm{Mbar}$ represents the Plasma Phase Transition (PPT) supposed to separate molecular from metallic hydrogen as computed by Saumon et al. (1995). The region in which hydrogen is predicted to be solid is represented as a dashed area. Lines showing the values of the parameters $\theta$ and $\Gamma$ (see text) are also shown. 
molecules, the energy of most of the electrons is not $k T$ nor $k T_{\mathrm{F}}$ so that its usefulness in that regime is limited. It can be seen from fig. 3.1 that the interiors of substellar objects are always characterized by $\theta<1$. It is never possible to assume that free electrons behave like a perfect gas.

Another important quantity is the coupling parameter, defined as the ratio of the Coulomb potential to the thermal energy:

$$
\Gamma=\frac{e^{2}}{a k T}=\frac{e^{2}}{k}\left(\frac{4 \pi}{3 \mu m_{\mathrm{u}}}\right)^{1 / 3} \frac{\rho^{1 / 3}}{T},
$$

where $a$ is the mean distance between nuclei. As $\Gamma$ increases due either to an increase of the density or to a decrease of the temperature, the Coulomb forces becomes more effective. With increasing densities, the system of ions eventually favors a non-random organization and becomes bound into a lattice system. This occurs for large values of $\Gamma(\sim 100)$. Figure 3.1 shows that substellar objects always have $\Gamma>1$ : the system is dominated by the repulsive coulombian potential between nuclei. However, we will be concerned with values of $\Gamma<50$, i.e. unlike white dwarfs, substellar objects are not expected to crystallize (this occurs for $\Gamma \gtrsim 180$ ). Hubbard (1968) was the first to show that Jupiter's interior should be hot enough for its interior to be fluid. It can also be seen in the phase diagram that it is the case of Saturn. For Uranus and Neptune, the situation is actually more complex because at large pressures they are not expected to contain hydrogen, but several studies show that ices in their interior should be fluid as well (e.g. Cavazzoni et al. 1999).

The largest fraction of the interior of brown dwarfs and giant planets is in a region in which hydrogen is metallic: the hydrogen molecules have been dissociated and ionized. The pressure inside this region can be expressed in the following form (e.g. Stevenson 1991):

$$
P=P_{\mathrm{e}}+P_{\mathrm{th}, \text { ion }}+P_{\text {coul }}+P_{\mathrm{ex}},
$$

where $P_{\mathrm{e}}$ represents the contribution from the electron gas, $P_{\text {th,ion }}$ the contribution from the thermallized ions, and $P_{\text {coul }}$ and $P_{\mathrm{ex}}$ are negative terms due to the Coulombian interactions of nuclei in the sea of electrons, and the reduction in electron-electron repulsion due to the exclusion principle, respectively. $P_{\text {coul }}$ is significant when $\Gamma$ becomes large. The exchange pressure $P_{\text {ex }}$ has to be taken into account for small values of $\theta$. Although quantitatively, the terms due to ions are important, most of the important physics and in particular the molecular/metallic transition is due to a difference in behavior of the electrons when the density rises.

\subsubsection{The degenerate electron gas}

In stars with masses larger than about $0.3 \mathrm{M}_{\odot}$ the electrons always behave with a near-maxwellian distribution of the momenta. However, for objects of lower interior temperatures, the Pauli exclusion principle yields a distribution which is determined by Fermi-Dirac statistics. The number of electrons in a volume $\mathrm{dV}$ and with an absolute of the momentum in $[p, p+d p]$ is:

$$
f(p) d p d V=\frac{8 \pi p^{2} d p d V}{h^{3}} \frac{1}{1+e^{E / k T-\psi}},
$$

where in the non-relativistic case $E=p^{2} /\left(2 m_{\mathrm{e}}\right)$ and $\psi$ is the degeneracy parameter. For $\psi \rightarrow-\infty$ the distribution is identical to the Maxwell-Boltzmann one. In the limit $\psi \rightarrow+\infty$ the electrons are said to be fully degenerate. 
The density of electrons, electronic pressure and internal energy can be obtained through integrations of that distribution:

$$
\begin{aligned}
n_{\mathrm{e}} & =\frac{8 \pi}{h^{3}} \int_{0}^{\infty} \frac{p^{2} d p}{1+e^{E / k T-\psi}} \\
P_{\mathrm{e}} & =\frac{8 \pi}{3 h^{3}} \int_{0}^{\infty} \frac{v p^{3} d p}{1+e^{E / k T-\psi}} \\
U_{\mathrm{e}} & =\frac{8 \pi}{h^{3}} \int_{0}^{\infty} \frac{E p^{2} d p}{1+e^{E / k T-\psi}}
\end{aligned}
$$

The degeneracy parameters $\psi$ obtained in the central region of substellar objects is relatively independent of the mass and age (to a factor $\sim 3$ ) and is of the order of $\psi \approx-30$ (e.g. Chabrier $\&$ Baraffe 2000). The combination of these low values of $\theta$ and $\psi$ thus implies that a significant fraction of the electrons are indeed degenerate.

Although this is not true of regions at lower pressures, we will find it instructive to use the relations for a fully degenerate electron gas for qualitative estimates. In the limit $\psi \rightarrow \infty$, one finds that the completely degenerate non-relativistic electron gas is such that (e.g. Kippenhahn \& Weigert 1991):

$$
\begin{aligned}
P_{\mathrm{e}} & =\frac{1}{20}\left(\frac{3}{\pi}\right)^{2 / 3} \frac{h^{2}}{m_{\mathrm{e}}} n_{\mathrm{e}}^{5 / 3} \\
& =1.0036 \times 10^{13}\left(\frac{\rho}{\mu_{\mathrm{e}}}\right)^{5 / 3}(\mathrm{cgs}) \\
U_{\mathrm{e}} & =\frac{3}{2} P_{\mathrm{e}}
\end{aligned}
$$

\subsubsection{Pressure ionization}

As seen in the phase diagram, hydrogen can become ionized due to increasing pressure instead of standard ionization at increasing temperature. Basically, this occurs when the degenerate electrons get a Fermi energy which is larger than that necessary to ionize hydrogen atoms. The approximate level at which this occurs can be estimated as follows.

First, it can be noted that both free and bound electrons have to obey the Pauli principle.

The energy of each electron is hence of the order $U_{\mathrm{e}} / n_{\mathrm{e}}$. For a set value of $n_{\mathrm{e}}$ a lower bound on $U_{\mathrm{e}}$ can be obtained by assuming full degeneracy (Eq. (3.11)). In order to become ionized this value has to become larger than the ionization potential of hydrogen, $u_{0}=13.6 \mathrm{eV}$. This occurs when

$$
n_{\mathrm{e}} \gtrsim\left(\frac{8 \pi}{3}\right)^{5 / 2}\left(\frac{5 m_{\mathrm{e}}}{4 \pi h^{2}}\right)^{3 / 2} u_{0}^{3 / 2},
$$

corresponding to an electronic pressure

$$
P_{\mathrm{e}} \gtrsim \frac{2}{3}\left(\frac{8 \pi}{3}\right)^{5 / 2}\left(\frac{5 m_{\mathrm{e}}}{4 \pi h^{2}}\right)^{3 / 2} u_{0}^{5 / 2} .
$$

Quantitatively, hydrogen metallization is then found to occur around $n_{\mathrm{e}} \sim 5 \times 10^{23} \mathrm{~cm}^{-3}, \rho \sim$ $0.8 \mathrm{~g} \mathrm{~cm}^{-3}$ and $P_{\mathrm{e}} \sim 7 \mathrm{Mbar}$. Even though crude assumptions were made, this is relatively close to more elaborate calculations. 
The same estimates can be used for helium ionization, assuming helium atoms are immersed in a sea of protons and electrons. Because $u_{0}=54.4 \mathrm{eV}$, the density and electronic pressure for helium ionization rise to $6.5 \mathrm{~g} \mathrm{~cm}^{-3}$ and $230 \mathrm{Mbar}$, respectively. However, at those very high densities, the distance between nuclei has become much smaller than the Bohr radius $\left(a_{0}=5.3 \times 10^{-9} \mathrm{~cm}\right)$. A very crude solution is to use an effective potential $u_{\text {eff }}=u_{0}\left(1-\left(a_{0} / d\right)^{2}\right)$ to account for the fact that the ionization energy is reduced due to the proximity to the other nuclei. The mean distance between hydrogen nuclei is $d \sim\left(3 / 4 \pi n_{\mathrm{e}}\right)^{1 / 3}$. Including that correction and solving iteratively Eq.(3.12), one finds that helium could ionize at a pressure as low as $P_{\mathrm{e}} \sim 17 \mathrm{Mbar}$. Applied to hydrogen, this procedure also leads to a reduced ionization pressure $P_{\mathrm{e}} \sim 2 \mathrm{Mbar}$.

The total pressure cannot be obtained through that method because one then needs to describe the system of ions. In the metallic regions of substellar objects, an order of magnitude estimate is that ions and electrons have similar contributions to the total pressure. Our assumption of full degeneracy in fact tends to overestimate the pressures at which the transition occurs. This can be understood by the fact that the Pauli distribution corresponds to the minimum energy state for a fixed density $n_{\mathrm{e}}$. Thermal effects have the tendency to move some of the electrons to higher energies, thereby favoring ionization. The transition from molecular to metallic hydrogen is therefore expected to occur at lower pressures and densities when the temperature is increased. Of course, these crude estimates are given for didactic purposes, but cannot replace a full treatment of this complex problem.

\subsection{Experiments and theoretical hydrogen EOSs}

\subsubsection{Reaching ultrahigh pressures: experimental results}

The high pressures and high temperatures typical of the interiors of giant planets can be achieved in the laboratory by shock-compression of a small sample of material. The shock is typically generated by a hypervelocity impactor or by a powerful laser. Measuring the thermodynamic properties of the compressed sample is quite difficult since such dynamical experiments last only 5 - $100 \mathrm{~ns}$ and the sample can be very small $\left(0.4-500 \mathrm{~mm}^{3}\right)$. For a given initial state of the sample, the family of shocked states that can be achieved follows a curve in the $(P, \rho, T)$ phase diagram known as a Hugoniot. The Hugoniot is one of the Rankine-Hugoniot relations that result from the conservation of energy, momentum, and matter flux across the shock front. Nearly all dynamical experiments on hydrogen and deuterium performed share the same cryogenic initial state and therefore measurements from different experiments can be directly compared. By reflection of the shock wave on a back plate made of a material stiffer than the sample, a double-shocked state can be achieved that reaches even higher pressures with a modest increase in temperature. Multiple shock reflections, known as shock reverberation, lead to a succession of compressed states that approach adiabatic compression.

Since 1995, deuterium has been the subject of intense experimental study using several independent techniques. ${ }^{1}$ Measurements of the pressure, density, temperature, reflectivity, electrical conductivity, and sound speed have been performed along the single-shock Hugoniot and, in some cases, along double-shock Hugoniots.

The most reliable experimental results come from experiments where the impactor is accelerated with a gas gun. This technique allows for larger samples $\left(\sim 500 \mathrm{~mm}^{3}\right)$ and longer lasting $(\sim 100 \mathrm{~ns})$ experiments but is generally limited to pressures below 1 Mbar. Pressures and densities have been

\footnotetext{
${ }^{1}$ Due to its higher density, deuterium is experimentally more advantageous than hydrogen because higher shock pressures can be achieved for a given impactor speed.
} 


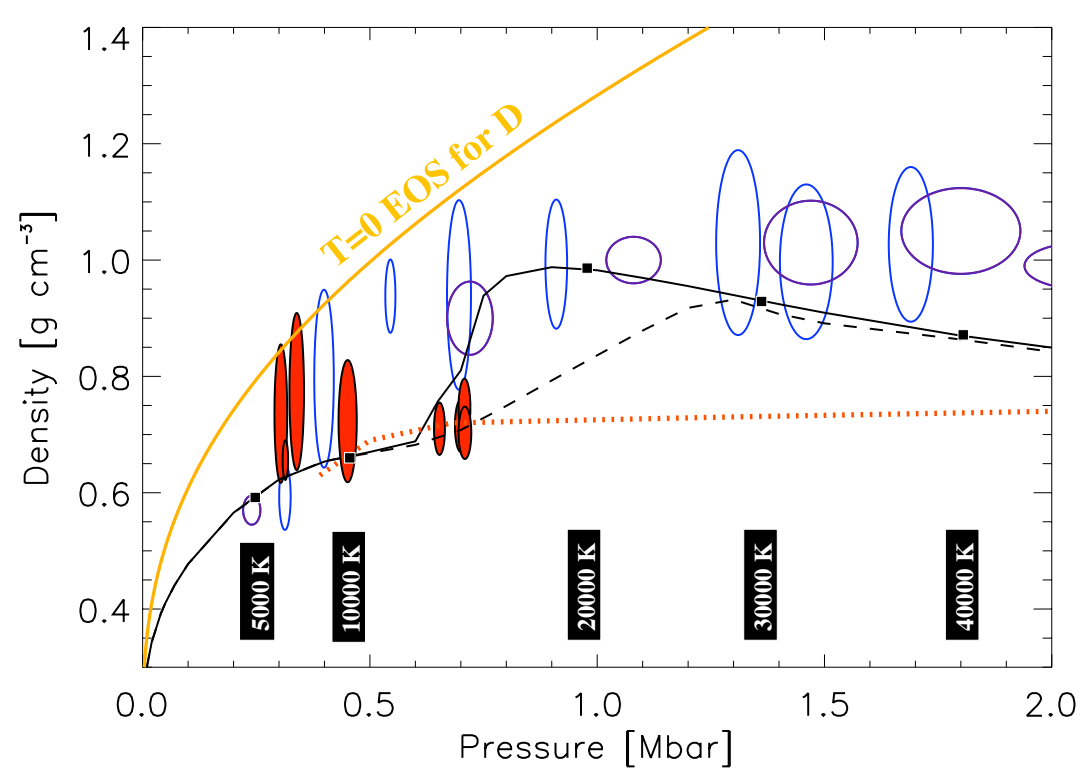

Figure 3.2: Comparison of experimental data and theoretical Hugoniot for deuterium (densities are twice larger than expected for hydrogen at any given pressure). Empty ellipses correspond to data points obtained from laser compression (Collins et al. 1998). Filled ellipses were obtained by magnetic compression (Knudson et al. 2001). Theoretical calculations are represented by lines. They are respectively: the "PPT" (solid) and "interpolated" (dashed) Saumon-Chabrier equations of state (Saumon, Chabrier \& Van Horn 1995), and a Path Integral Monte Carlo EOS (Militzer \& Ceperley 2000). The solid line to the left shows the $\mathrm{T}=0$ equation of state for $\mathrm{D}_{2}$ as determined by an exp-6 potential fit to diamond-anvil cell measurements (Hemley et al. 1990). The temperatures along the Hugoniot have been calculated using the PPT-EOS. [From Guillot et al. 2003].

measured along the single-shock Hugoniot up to $0.2 \mathrm{Mbar}$ and along the double-shock Hugoniot up to $0.8 \mathrm{Mbar}$ (Nellis et al. 1983). The reshocked states reproduce the $(P, T)$ conditions of the molecular hydrogen envelope of Jupiter and provide a direct probe of the thermodynamics of hydrogen.

Under conditions where the dissociation of molecules becomes significant, the temperature becomes a sensitive test of the EOS. Processes that can absorb substantial amounts of energy like dissociation and ionization result in relatively cool temperatures and higher degrees of compression for a given pressure along the Hugoniot. In the absence of such processes, the energy of the shock is expended mostly in the kinetic degrees of freedom with a corresponding increase in temperature. The temperature of double-shocked deuterium (Holmes, Ross \& Nellis 1995) was found to be lower than all EOS predictions by about 30-40\%, indicating that dissociation plays a more important role than predicted by contemporaneous models.

Finally, the sound speed has been measured along the Hugoniot in gas gun experiments up to $0.28 \mathrm{Mbar}$ (N. C. Holmes, priv. comm.). Since it is a derivative of the pressure, the sound speed is a sensitive test of EOS models with the advantage of being measurable very reliably.

With powerful lasers, deuterium can be shocked to much higher pressures than with gas guns but the small sample size and the very short duration of the experiments make accurate diagnostics very challenging. The $(P, \rho, T)$ single shock Hugoniot has been measured recently up to 3.5 Mbar with 
the NOVA Laser Facility (Da Silva et al. 1997; Collins et al. 1998; Collins et al. 2001), reaching a maximum density of $\sim 1 \mathrm{~g} \mathrm{~cm}^{-3}$ at $\sim 1 \mathrm{Mbar}$ (fig. 3.2). Such a high compressibility was not anticipated by most EOS models and this work sparked the current interest in the thermodynamics of warm dense hydrogen as well as controversy, both on the theoretical and experimental fronts. The reflectivity of shocked deuterium reaches about $60 \%$ for pressures above $0.5 \mathrm{Mbar}$ along the Hugoniot (Celliers et al. 2000), a value indicative of a large density of free electrons and of a high electric conductivity characteristic of fluid metallic hydrogen. Second-shock compression up to $6 \mathrm{Mbar}$ with the Nike laser give results in agreement with the NOVA $(P, \rho)$ data (Mostovych et al. 2000). On the other hand, Knudson et al. (2001) used a magnetic Z-accelerator to accelerate impactors to very high velocities. Their single-shock Hugoniot agrees well with the NOVA data for $P \lesssim 0.4 \mathrm{Mbar}$ but it is not as compressible at higher pressures, reaching a density of only $\sim 0.7 \mathrm{~g} \mathrm{~cm}^{-3}$ at $0.7 \mathrm{Mbar}$ (fig. 3.2).

\subsubsection{Hydrogen: EOS calculations}

While the temperatures obtained along the single-shock Hugoniot rapidly become much higher than those inside Jupiter at the same pressure (fig. 3.3), these measurements provide very important, and heretofore unavailable tests of equations of state in the 0.5 to 5 Mbar range where pressure ionization of hydrogen occurs. Conversely, EOS models can be used to compute the various physical quantities measured in the lab and to interpret the experimental results.

Theoretical single-shock Hugoniots computed from a wide variety of EOS models basically fall into two groups. First principle calculations (e.g. Militzer \& Ceperley 2000; Lenosky et al. 1997, 2000; Galli et al. 2000) all predict a rather stiff Hugoniot that is in general agreement with the Z-accelerator data of Knudson et al. (2001). This is illustrated in fig. 3.2 by the Path Integral Monte Carlo calculation of Militzer \& Ceperley (2000). On the other hand, models that are partly calibrated with experimental data (Saumon, Chabrier \& Van Horn 1995; Ross 1998; Saumon et al. 2000, Ross \& Yang 2001), obtain a generally good agreement with the NOVA data (fig. 3.2; Collins et al. 1998). Interestingly, the standard SESAME EOS of deuterium (Kerley 1972) predicts a Hugoniot that generally agrees with the much more sophisticated $a b$ initio calculations.

Our study of a number of theoretical Hugoniots shows that EOS that have been fitted to the gas gun single- and double-shock $(P, \rho, T)$ data of Nellis et al. (1983) and Holmes et al. (1995) all taken below $0.8 \mathrm{Mbar}$ and $5300 \mathrm{~K}$ - reproduce the high compression of the NOVA data (Collins et al. 1998) and the sound speed data along the single shock (N. C. Holmes, priv. comm.). On the other hand, the first-principle calculations generally agree with the stiffer Hugoniot of Knudson et al. (2001) and cannot reproduce the high compression of the NOVA data. They also fail to reproduce the double-shock temperatures and the sound speed measurements. Some of the $a b$ initio calculations disagree with the low-pressure gas gun data (e.g. Lenosky et al. 2000). On the one hand, the Knudson et al. (2001) data and nearly all first-principle EOS calculations are in good agreement with each other. On the other hand, more heuristic EOS models clearly show that four independent EOS experiments (second-shock temperature, sound speed, the NOVA single shock and the Nike double shock) are fully consistent with each other but neither with first principle calculations nor the Knudson et al. (2001) data. Both the high compressibility of the NOVA Hugoniot and the low gas-gun reshock temperatures can be explained by the absorption of the shock energy resulting from molecular dissociation.

This polarization of EOS calculations along different data sets has created a lively debate and is stimulating much additional (and challenging) experimental and theoretical work. The EOS of hydrogen in the 0.5 to $5 \mathrm{Mbar}$ regime, where it is transformed from an insulating molecular fluid to 


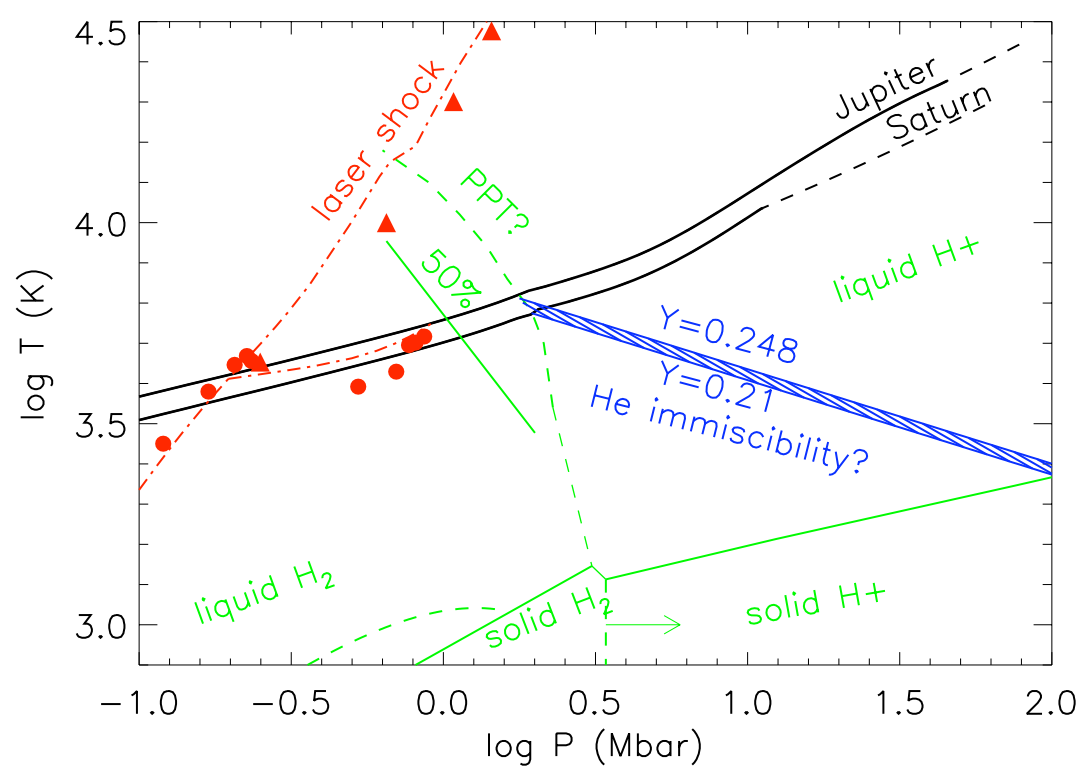

Figure 3.3: Hydrogen phase diagram, with interior profiles of present-day Jupiter and Saturn overlaid, and with some experimental data shown. The boundary between liquid $\mathrm{H}_{2}$ and solid $\mathrm{H}_{2}$ is somewhat uncertain in the Mbar pressure range (2 estimates are shown), but is not relevant to Jupiter. The laser shock measurements of Collins et al. (2001) and the gas-gun measurements of Holmes et al. (1995) are shown as triangles and filled circles in the upper left-hand corner, respectively. Single- and double-shock hydrogen Hugoniots calculated by Saumon et al. (2000) are shown as dot-dashed lines in the same region of the plot. The solid line labeled "50\%" shows where $50 \%$ of molecular dissociation is obtained in the model of Ross (1998).

a conducting liquid metal remains uncertain to a level that is significant for modeling the interior of Jupiter. The recent progress in this area as been very beneficial, however, as it appears that the current data and models bracket the actual EOS of hydrogen.

In order to model Jupiter's interior with confidence, a careful study of the uncertainties arising from the EOS would be required. This is not presently available, but fig. 3.2 shows that this can be crudely approximated by using the "interpolated" and the "PPT" equations of state of Saumon, Chabrier \& Van Horn (1995) even though they do not fit the experimental data well. However, large uncertainties in density along the Hugoniot at 1 Mbar $(\sim 30 \%)$ result in much smaller differences along the Jupiter adiabat $(\sim 8 \%)$. The effects on the inferred core mass and the mass of heavy elements in Jupiter and Saturn are discussed later on.

\section{A Plasma Phase Transition?}

We have seen that hydrogen undergoes a transition from a low-pressure molecular insulating fluid to a high-pressure conductive fluid. Is the transition continuous, as is the case for temperature ionization, or rather a first order phase transition (the so-called Plasma Phase Transition, or PPT) with discontinuities in density and entropy across the coexistence curve? Such a first-order 
transition was first suggested by Wigner \& Huntington (1935) on the basis of the different nature of the interaction potentials in metals (a weakly repulsive, screened Coulomb potential) and in insulators (a strongly repulsive "hard-sphere" potential).

The PPT has not been observed experimentally in hydrogen (i.e. there is no evidence for the expected discontinuities), but it can be argued that the gas-gun experiments have not reached high enough pressures, and that laser-shocks may be supercritical. Note for example that using the new data, the critical point for the PPT computed by Saumon et al. (2000a) is lower ( $T \approx 14600 \mathrm{~K}$; $P \approx 0.73 \mathrm{Mbar})$ than shown in fig. 3.3. The PPT is predicted by some of the more heuristic "chemical picture" EOS models (Saumon et al. 1995 and references therein) and Beule et al. (1999). On the other hand, none of the first-principle EOS calculations show evidence for a first order phase transition in warm dense hydrogen. This can be seen in fig. 3.4 which shows a continuous variation of the proton-proton pair correlation function as a function of density and temperature obtained by Militzer \& Ceperley (2001). The figure indicates that $\mathrm{H}_{2}$ molecules are present at low temperatures and densities, as seen by the peak at $\sim 0.8 \AA$, and the fact that the correlation function goes to zero at larger distances. As one increases the density, the correlation function becomes non-zero everywhere except close to a proton, indicating that hydrogen has been dissociated.

If present, the PPT would have significant consequences for the structure of Jupiter, Saturn, and low-entropy extrasolar giant planets. Its main effect would be to create an impenetrable barrier for convection between the molecular and metallic hydrogen parts of the envelope, affecting the mixing of chemical species (Stevenson \& Salpeter 1977b). The thermodynamic conditions of phase equilibrium imply that the chemical composition across the PPT must be discontinuous (Landau \& Lifschitz 1969), with the consequence that atmospheric abundances of all elements would no longer be indicative of their bulk abundance in the planet. In addition, as the planet cools, a fraction of the mass of the envelope is converted from one phase to the other with an associated latent heat release (or absorption). The effect on the evolution is not very pronounced for a latent heat of $\sim 0.5 k_{B}$ per proton (Saumon et al. 1992).

\subsection{Other elements}

\subsubsection{Approximate equations of state}

An equation of state has been computed for helium by Saumon et al. (1995), but it is less sophisticated (realistic?) than the hydrogen EOS. This shouldn't affect the results too much because in a solar composition mixture, hydrogen represents about $90 \%$ of the atoms, and helium only about $10 \%$. The consequent EOS for the hydrogen-helium mixture is then calculated using the additive volume rule:

$$
\begin{aligned}
& \rho^{-1}=(1-Y) \rho_{\mathrm{H}}^{-1}+Y \rho_{\mathrm{He}}^{-1} \\
& U=(1-Y) U_{\mathrm{H}}+Y U_{\mathrm{He}} \\
& S=(1-Y) S_{\mathrm{H}}+Y S_{\mathrm{He}}+S_{\mathrm{mix}}(Y),
\end{aligned}
$$

where $S_{\text {mix }}$ is the entropy of mixing, and all quantities depend on $P$ and $T$. This method implicitly neglects any interactions between hydrogen and helium.

For other elements, the treatment is even more approximate. Zharkov (1984) suggests a fit in the form of a zero temperature pressure plus a thermal component:

$$
P(\rho, T)=P(\rho, 0)+\frac{3 \mathcal{R} T}{\bar{A}} \rho^{\gamma},
$$




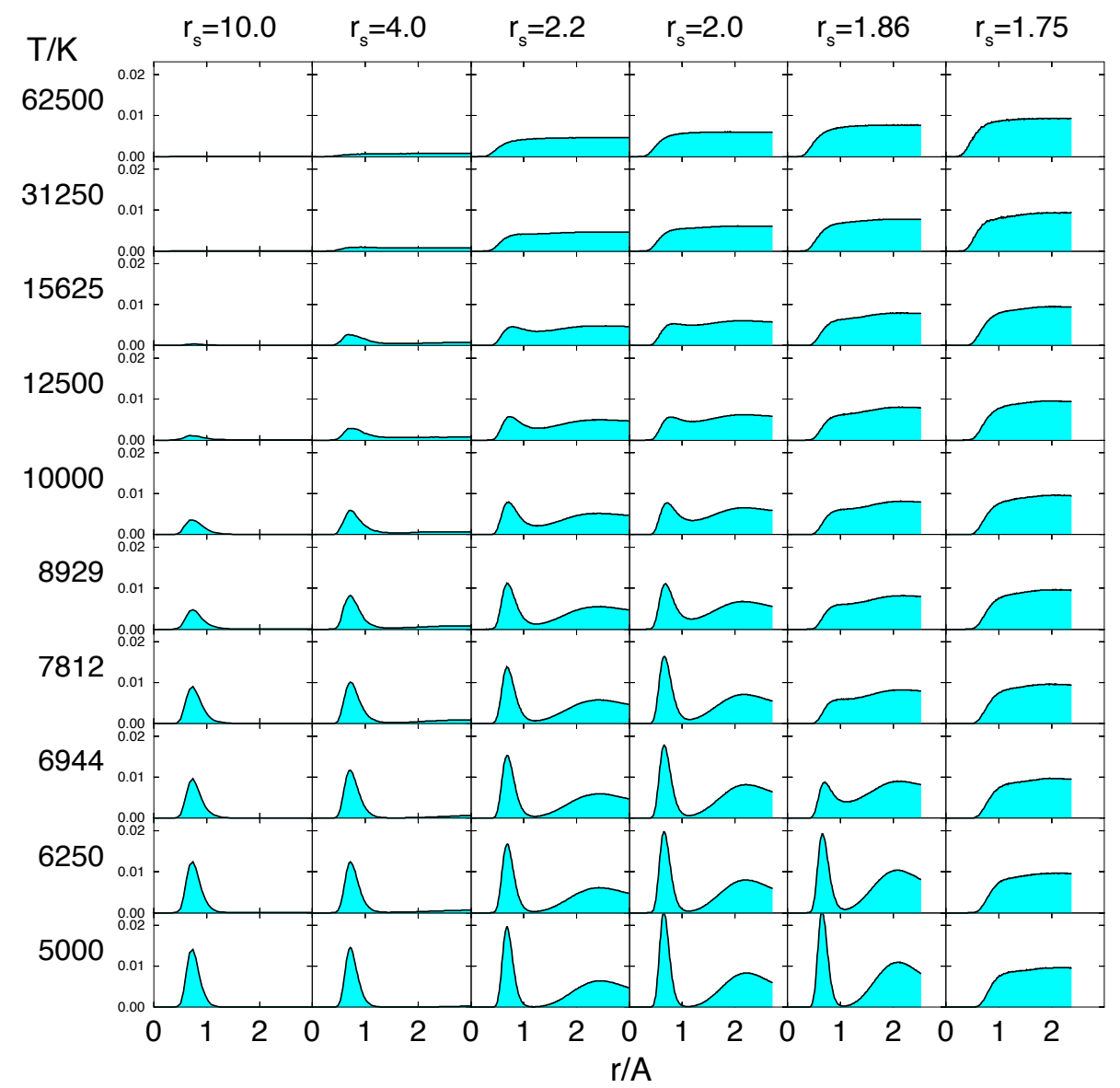

Figure 3.4: Proton-proton pair correlation function multiplied by the density $n$ as a function of interparticle distance $r$ (in Angstrom). The columns correspond, from left to right, to decreasing values of the density parameter $r_{s}=a_{0} / a_{e}$ (increasing density $\rho ; a_{0}$ is Bohr's radius, $a_{e}$ is the mean electronic distance). The rows correspond to temperatures increasing from $5000 \mathrm{~K}$ (bottom) to $62500 \mathrm{~K}$ (top). [Courtesy of B. Militzer; see also Militzer \& Ceperley 2001]. 
where $\gamma$ is the Grüneisen parameter (generally of order $\sim 1$ ). Fits for various elements are given by Zharkov (1984). Similar approximate relations are also provided by Hubbard et al. (1995).

A fit to the densities of "ices" (initially a mixture of water, methane and ammonia) and "rocks" at high pressures and planetary temperatures is provided by Hubbard \& Marley (1989) based on experimental Hugoniot data:

$$
\begin{aligned}
\text { "ices" } & P=\rho^{3.719} \exp \left(-2.756-0.271 \rho+0.00701 \rho^{2}\right), \\
\text { "rocks" } & P=\rho^{4.406} \exp \left(-6.579-0.176 \rho+0.00202 \rho^{2}\right),
\end{aligned}
$$

where $P$ is the pressure in megabars and $\rho$ is the density in $\mathrm{g} \cdot \mathrm{cm}^{-3}$. This fit is valid in the pressure range $0.1 \mathrm{Mbar}<P<8 \mathrm{Mbar}$.

\subsubsection{Miscibility of elements in hydrogen}

As first proposed for Jupiter and Saturn by Smoluchowski (1967) and Salpeter (1973), helium can undergo a phase separation from hydrogen: at low temperatures, helium (or other elements) can become insoluble and form droplets. Under the action of gravity, these droplets will tend to fall toward the central regions of the planet.

Physically, a phase separation arises in a binary mixture of concentration $c$ when the second derivative of the Gibbs free energy $\partial^{2} G / \partial c^{2}<0$. The two concentrations $c_{1}$ and $c_{2}$ of equal chemical potentials $(\partial G / \partial c)\left(c_{1}\right)=(\partial G / \partial c)\left(c_{2}\right)$ correspond to the concentration of the droplets and the environment which are in equilibrium. The lower the temperature, the closer $c_{1}$ and $c_{2}$ are to 0 and 1 , respectively.

Of course, when calculating the miscibility of hydrogen-helium mixture, both hydrogen, helium and their interactions should be accounted for. Given the difficulty in modeling the EOS for hydrogen alone, it may not be so surprising that the question of the helium phase separation in the giant planets is still unsolved.

One approach has been to calculate the hydrogen-helium phase diagram assuming complete ionization. In that case, critical temperatures of order $8000 \mathrm{~K}$ at $2 \mathrm{Mbar}$ can be calculated (see Stevenson 1982). Even more importantly, this leads to a critical temperature that decreases with increasing pressure. The consequence is that (i) this would imply that a phase separation has occurred in Jupiter, and earlier in Saturn, as suggested by the abundance of helium measured in the atmosphere (see section 1); (ii) helium would be most insoluble near the molecular/metallic transition.

Other calculations have been attempted in the local density approximation (physical picture). Earlier work (Klepeis et al. 1992) suggested a unrealistically high critical temperature (40000 K at $10.5 \mathrm{Mbar}$ ). However, a more careful study by Pfaffenzeller et al. (1995) with the same basic technique led to a lower critical temperature (less than $5000 \mathrm{~K}$ at $4 \mathrm{Mbar}$ ). This value would imply no demixing of helium in Jupiter and Saturn. More importantly, the work of Pfaffenzeller et al. implies a critical temperature that increases with pressure. This can be explained if hydrogen is still not fully ionized at the pressures considered (4 to $24 \mathrm{Mbar}$ ), which seems difficult to reconcile with the more standard hydrogen EOSs. Another problem of the work of Pfaffenzeller et al. is that it does not recover the fully ionized limit. If the critical temperature increases with pressure, this would open the possibility that helium separates from hydrogen over an extended fraction of the planetary radius, with significant consequences for the interior and evolution models.

Other elements are also expected to separate from hydrogen if the temperature is low enough. However, the only estimates are for fully ionized mixtures. Table 3.1 shows critical temperatures and concentrations for the separation of various mixtures, as estimated by Stevenson (1976b). The 
Table 3.1: Separation of fully ionized mixtures

\begin{tabular}{ccc}
\hline \hline Mixture & $T_{\mathrm{c}}[K]$ & $c_{\mathrm{c}}$ \\
\hline $\mathrm{H}-\mathrm{Li}$ & $1.4 \times 10^{4}$ & 0.18 \\
$\mathrm{H}-\mathrm{C}$ & $1.1 \times 10^{5}$ & 0.086 \\
$\mathrm{H}-\mathrm{O}$ & $2.6 \times 10^{5}$ & 0.064 \\
$\mathrm{H}-\mathrm{Fe}$ & $5.5 \times 10^{6}$ & 0.019 \\
\hline
\end{tabular}

low temperatures for demixing are due to the different coulombian potential for hydrogen and ions of progressively larger charges. As for helium however, these elements are not expected to be fully ionized which severely limits the applicability of these estimates to substellar objects. 


\section{Chapter 4}

\section{Opacities \& heat transport}

We have seen that modeling the interiors of substellar objects requires to be able to calculate the temperature gradient $\nabla_{T}$ at each level. This necessitates to know how energy is transported. Three processes can contribute to this transport: radiation, conduction and convection.

\subsection{Radiation absorption - basic considerations}

Let us consider a ray of radiation whose initial intensity is $I_{0 \nu}$ as a function of frequency $\nu$ passing through a medium of density $\rho$ on a distance $l$. The final intensity is then

$$
I_{\nu}=I_{0 \nu} e^{-\kappa_{\nu} \rho l},
$$

where $1 / \kappa_{\nu} \rho$ corresponds to the mean free path of photons of frequency $\nu$, and $\kappa_{\nu}$ is the monochromatic opacity. As example of possible values in the interiors of giant planets and brown dwarfs are $\kappa \sim 1 \mathrm{~cm}^{2} \mathrm{~g}^{-1}, \rho \sim 10^{-2}$ leads to a photon mean free path of $l_{\mathrm{ph}} \sim 1$ meter.

As can be intuited from this very small mean free path, radiation in the interior is almost isotropic. In order to show that, let us consider the radial temperature difference between two levels separated by the photon mean free path:

$$
\Delta T=l_{\mathrm{ph}} \frac{d T}{d r} .
$$

The temperature lapse rate $d T / d r$ cannot be calculated a priori. However, typical values for the Jupiter's interior are $d T / d r \approx 10^{4} / 10^{9} \mathrm{~K} \mathrm{~cm}^{-1}$, and $l_{\mathrm{ph}} \approx 10^{2} \mathrm{~cm}$ implying $\Delta T \approx 10^{-3} \mathrm{~K}$. Since the energy density is proportional to $T^{4}$, the anisotropy has to be of the order $4 \Delta T / T$. Using the previous estimate and $T \approx 10^{4} \mathrm{~K}$, one can see that it is of the order of $4 \times 10^{-7}$, i.e. most of the interiors of giant planets and brown dwarfs can be considered as isotropic when radiation is concerned. Note that this is not the case near the photospheres of these objects, where photons can escape to space and $l_{\mathrm{ph}}$ becomes large. In that case, the full radiative transfer equation has to be solved. We refer the reader to available textbooks on the subject for further information on that problem (e.g. Goody \& Yung 1989).

For modeling the interior, it is therefore justified to use the diffusion approximation, : radiation then obeys a standard diffusion equation:

$$
\mathbf{j}=-D \nabla n,
$$


where $\mathbf{j}$ is the radiation flux, $D$ is the diffusion coefficient, which can be shown to be equal to $c l_{\mathrm{ph}} / 3$ (e.g. Clayton 1968) and $n$ represent the energy density $U_{\nu}$. Because all the variables only vary radially, we can rewrite the diffusion equation as:

$$
F_{\nu}=-\frac{c}{3 \kappa_{\nu} \rho} \frac{\partial U_{\nu}}{\partial r}
$$

where $F_{\nu}$ is the net radial flux per unit wavelength ${ }^{1}$.

In this approximation, the energy density at each level of temperature $T$ is proportional to the black body function $B_{\nu}(T)$ :

$$
U_{\nu}(T)=\frac{4 \pi}{c} B_{\nu}(T)=\frac{8 \pi h}{c^{3}} \frac{\nu^{3}}{h \nu / k T-1}
$$

The total radial flux can then be obtained by integrating over all frequencies:

$$
F=-\left[\frac{4 \pi}{3 \rho} \int_{0}^{\infty} \frac{1}{\kappa_{\nu}} \frac{\partial B_{\nu}}{\partial T} d \nu\right] \frac{\partial T}{\partial r} .
$$

It is thus convenient to define the Rosseland mean opacity as

$$
\kappa_{\mathrm{R}}=\left[\frac{\pi}{a c T^{3}} \int_{0}^{\infty} \frac{1}{\kappa_{\nu}} \frac{\partial B_{\nu}}{\partial T} d \nu\right]^{-1} .
$$

Note that $\kappa_{\mathrm{R}}$ is a harmonic average of the opacity, weighted by a function which is close to a blackbody function and peaks at $\nu=4 k T / h$ (or equivalently $\sigma=2.78 T$ where $\sigma$ is expressed in $\mathrm{cm}^{-1}$ and $T$ in Kelvins). This has crucial consequences for its calculation, as spectral regions for which the monochromatic opacity is the smallest will tend to have the most important contribution to the mean. Physically, this can be interpreted by the fact that the cooling of any given layer in the star/planet will be governed by the photons which have the longest mean free path. Numerically, this implies that regions where the opacities are least known will have potentially very important contributions and that the final accuracy is extremely hard to estimate.

On the other hand, in a radiative or conductive environment, the temperature gradient will be directly given by the intrinsic luminosity, as can be seen from eqs. (4.3) and (4.4):

$$
\frac{\partial T}{\partial r}=-\frac{3}{16 \pi a c} \frac{\kappa_{\mathrm{R}} \rho L}{r^{2} T^{3}} .
$$

In a radiative/conductive region, the temperature profile is hence steeper when the luminosity to be transported is larger. In the limit of a zero luminosity, it becomes isothermal as can be expected from thermodynamic principles.

\subsection{Rosseland opacities}

\subsubsection{Absorption of a zero-metallicity gas}

The contribution of hydrogen and helium to the overall opacities is often relatively small but fundamental, due to the nature of the Rosseland mean. At the pressures (bars or more) and

\footnotetext{
${ }^{1}$ Note that when including rotation, this equation is not strictly valid any more: the surfaces of constant intrinsic flux then tend to become more spherical than those of constant pressure. In a radiative environment, this gives rise to a slow meridional circulation also known as the Eddington-Sweet circulation.
} 
temperatures (100s to $1000 \mathrm{~s} \mathrm{~K}$ ) of interest, these elements mostly have continuum opacity and therefore avoid any divergence of eq. (4.4).

One of the most complete and useful work on the subject so far is certainly that of Lenzuni et al. (1991). I refer the reader to that paper for details on this problem. In this course, the materials that will be considered is relatively cool and at high density, implying that the main absorption sources are:

$\mathrm{H}_{2}-\mathrm{H}_{2}$ and $\mathrm{H}_{2}-\mathrm{He}$ collision-induced absorption (CIA): $\mathrm{H}_{2}$ and He in their ground state have no electric dipole and mainly absorb during collisions. The $\mathrm{H}_{2}$ molecule has three degrees of freedom: translation, rotation ${ }^{2}$ and vibration ${ }^{3}$. The largest energy transitions are between the vibrational bands, while the rotational bands imply a finer structure whose main consequence is to broaden these bands. The detailed calculation and structure is complex, especially in the case of the $\mathrm{H}_{2}-$ $\mathrm{H}_{2}$ collision (4 other quantum numbers are then required to describe the state of the supermolecule), but to simplify it is dominated by 4 almost evenly spaced absorption bands (transitions $v: 0 \rightarrow 0$ to $v: 0 \rightarrow 3$ ) between 0 and $14000 \mathrm{~cm}^{-1}$. (See Borysow et al. 2000; Borysow 1992 and references therein).

$H^{-}$bound-free absorption: At high enough densities, the abundance of the $\mathrm{H}^{-}$ion can become non-negligible. In this case, photons of sufficiently high energy can dissociate the ion into a hydrogen atom and a free electron. The absorption rapidly rise with increasing wavenumbers to reach a maximum at 1 micron. At higher wavenumbers (energies) it slowly decreases.

$\mathrm{H}_{2}^{-}$free-free absorption: At very high densities, free electrons can "feel" the potential of the neutral $\mathrm{H}_{2}$ molecule and therefore act as a superparticule which can absorb radiation. The crosssection for this reaction is a rapidly decreasing function of wavenumber.

Rayleigh scattering by $\mathrm{H}_{2}$ : Although this is not real absorption, Rayleigh scattering is very important for limiting the propagation of high energy radiation due to its $1 / \lambda^{4}$ dependency.

\subsubsection{Molecular line opacities}

Due to the relatively low temperatures and high pressures encountered in regions where radiative heat transport matters, the opacity is dominated by molecular absorption. At low temperatures, the dominant molecules are $\mathrm{H}_{2} \mathrm{O}, \mathrm{CH}_{4}$ and $\mathrm{NH}_{3}$. For hotter objects $\mathrm{CH}_{4}$ transforms into $\mathrm{CO}$, and then $\mathrm{TiO}$ and $\mathrm{VO}$, two important absorbers in the stellar regime appear (see Fegley \& Lodders 1994, 1996; Lodders 1999).

Due to the complexity of the rotation and vibration modes of these molecules, one often has to rely on experimental measurements. Those can consist of measurements of mean absorptions in frequency intervals. These are however limited to a fixed number of pressures and temperature at which the measurements have been done. Another approach chosen for example for the GEISA and HITRAN data base is to measure the intensity of the largest possible number of lines. The absorption at any temperature and pressure of a given compound can then theoretically be calculated from the following relation:

$$
\kappa_{\nu}(T, P)=\sum_{i} I_{i}\left(T_{0}, P_{0}\right)\left[\frac{1-e^{h \nu / k T}}{1-e^{h \nu / k T_{0}}}\right]\left[\frac{Q(T)}{Q\left(T_{0}\right)} e^{-\frac{E_{i}}{k}\left(\frac{1}{T}-\frac{1}{T_{0}}\right)}\right] L_{\nu}\left(T, P, \nu_{i}\right)
$$

\footnotetext{
${ }^{2}$ Approximately, $E_{\text {rot }} \sim \hbar^{2} / 2 \operatorname{Ij}(j+1)$ where $I$ is the molecule's moment of inertia and $j$ the rotational quantum number.

${ }^{3} E_{\mathrm{vib}} \sim \hbar \omega_{\mathrm{osc}}(v+1 / 2)$ where $\omega_{\mathrm{osc}}$ is the vibration frequency of the equivalent harmonic oscillator and $v$ the vibrational quantum number.
} 
where the monochromatic opacity $\kappa_{\nu}$ and the observed intensity of the line $i$ are generally given in $\mathrm{cm}^{2}$ molec $^{-1}$ and the measured quantities have been obtained at temperature $T_{0}$ and pressure $P_{0}$. The ratio of exponential corresponds to induced emission. $Q(T)$ is the partition function at temperature $T, E_{i}$ the energy of the level from which the observed line $i$ comes from, and therefore the second term in square brackets is the ratio of the population of the initial energy level between temperature $T$ and $T_{0}$. The line profile is $L_{\nu}$ and this function is such that $\int_{0}^{\infty} L_{\nu} d \nu=1$.

Although theoretically reasonable, one of the main drawback of this approach is the fact that the extrapolation to high temperatures involves excited energy level transitions which are extremely difficult to detect at room temperatures. The problem of formula (4.6) is therefore that the population of energy levels corresponding to known lines decreases whereas the population of unknown excited levels increases. This problem, known as the "hot band" problem eventually leads to a strong (and false) decrease of the absorption with increasing temperature.

In recent years, progresses in computational power have lead to very interesting advances in $a b$ initio calculations. These calculation predict the entire energy levels of a given molecule and can therefore yield the absorption spectrum at all temperatures and pressures. These kind of calculations have been successfully applied to diatomic molecules such as $\mathrm{TiO}, \mathrm{CO}, \mathrm{VO}$...etc for quite a few years, using the principles of the harmonic oscillator. The case of the linear molecules as HCN has also been solved after that. However it is only relatively recently that convincing calculations have been performed for more complex molecules such as $\mathrm{H}_{2} \mathrm{O}$. Other important molecules in the context of cool objects $\left(T_{\text {eff }} \lesssim 1200 \mathrm{~K}\right)$ that still resist are $\mathrm{CH}_{4}$ and $\mathrm{NH}_{3}$. In the absence of crucial data for these molecules, one has to rely on hazardous extrapolation of experimental data.

\subsubsection{Line profiles}

In the case of stellar atmospheres, the problem of the profile of absorption lines is relatively straightforward. Because the medium is at relatively high temperatures and low densities the absorption of a molecule away from the center of a line is due to the Doppler shift of the radiation as seen by the absorber. The Doppler line profile is written as a function of wavenumber $(\sigma=\nu / c)$ :

$$
L_{\sigma}\left(T, \sigma_{0}\right)=\frac{e^{-\left(\sigma-\sigma_{0}\right)^{2} / \Delta \sigma_{\mathrm{D}}^{2}}}{\Delta \sigma_{\mathrm{D}} \sqrt{\pi}},
$$

and the line halfwidth is

$$
\Delta \sigma_{\mathrm{D}}=\frac{\sigma}{c} \sqrt{\frac{2 k T}{m}}
$$

where $m$ is the mean molecular mass.

In the case of most substellar objects, the cooler and denser conditions which prevail imply a different kind of broadening which is dominated by the effect of collisions: because the energy levels are populated only for finite periods of time (due to excitations/deexcitations caused by collisions), the transition cannot have a unique frequency. This gives rise to the so-called Lorentzian line profile, which is

$$
L_{\sigma}\left(T, P, \sigma_{0}\right)=\frac{\Delta \sigma_{\mathrm{L}}}{\left(\Delta \sigma_{\mathrm{L}}\right)^{2}+\left(\sigma-\sigma_{0}\right)^{2}},
$$

and the Lorentzian half-width then depends on details of the physics of microscopic collisions. In general, it can be approximated by the following perfect gas approximation

$$
\Delta \sigma_{\mathrm{L}} \approx \sigma_{0} \frac{P}{\sqrt{T}}
$$


and $\sigma_{0}$ depends on the line considered. This value can be experimentally determined at room temperature, but when using ab initio calculation it is generally set to a fixed value corresponding to the mean of the observed ones.

The use of the Doppler line broadening can be justified when its halfwidth value is larger than that of a Lorenztian profile, i.e. when

$$
T \gtrsim 7000 \mathrm{~K}\left(\frac{\rho}{10^{-4} \mathrm{~g} \mathrm{~cm}^{-3}}\right) \text {. }
$$

At significantly lower temperature and/or larger densities, one is justified to use a pure Lorentzian profile. In between, the Voigt profile is a combination of the two:

$$
L_{\sigma}\left(T, P, \sigma_{0}\right)=\int_{-\infty}^{+\infty} \frac{\Delta \sigma_{\mathrm{L}}}{\left(\Delta \sigma_{\mathrm{L}}\right)^{2}+\left(\sigma-\sigma_{0}-u \frac{\sigma_{0}}{c}\right)^{2}}\left(\frac{m}{2 \pi k T}\right) e^{-m u^{2} / 2 k T} d u .
$$

The Lorentzian (or Voigt) broadening is intrinsically more complicated than the Doppler one due to the additional pressure dependency and the a priori unknown halfwidth. It is also more complicated due to its slow decay compared to the Doppler profile.

A cutoff to the Lorentzian profile is generally used first because it is computationally much less intensive. It has also been empirically verified that synthetic spectra of the giant planets generally fit the observations better when using a cutoff. This is for example the case of the $5 \mu \mathrm{m}$ spectrum of Jupiter, modeled by Kunde et al. (1982) using a cutoff of $\Delta \sigma_{\text {cutoff }} \sim 120 \mathrm{~cm}^{-1}$. Last but not least, there are theoretical grounds for which the Lorentzian profile should fail far from the line center.

The Lorentzian "core" is indeed a result of the impact approximation: it is valid when the collision time is large compared to the characteristic time of the transition:

$$
\frac{r_{\mathrm{c}}}{v_{\mathrm{c}}}=\tau_{\mathrm{col}} \gtrsim \tau_{\omega}=\frac{1}{2 \pi c\left|\omega-\omega_{0}\right|},
$$

where $r_{\mathrm{c}}$ and $v_{\mathrm{c}}$ are the mean radius and velocity at closest approach during collisions. Further from the line center, the impact approximation fails and a faster exponential decay should prevail (Birnbaum 1979). This simplification was indeed used by Guillot et al. (1994a) to predict a line cutoff proportional to $\sqrt{T}$ around $100 \mathrm{~cm}^{-1}$ at $T=200 \mathrm{~K}$, consistent with spectroscopic models of Jupiter's atmosphere.

More generally, the Lorentzian profile is known to fail in a variety of conditions. Both superlorentzian and sublorentzian profiles can be observed, and spectral lines can even be shifted due to microscopic interactions and line mixing. However, a surprising result of the recent years is that, at least in the case of alkali metals, far wings can still be of significance much beyond expectation. In the case of $\mathrm{Na}$ lines in the visible spectral region, Nefedov et al. (1999) find that the expected exponential cutoff occurs for $\Delta \sigma \gg 1000 \mathrm{~cm}^{-1}$. Using a Lorentzian profile convoluted with an exponentially-decaying function, Burrows et al. (2000a) find that alkali metals, and especially the potassium doublet at $0.77 \mu \mathrm{m}$ can explain the absence of flux emitted by brown dwarfs in the visible and the slope of the spectrum for wavelengths shorter than $1 \mu \mathrm{m}$.

The consequences of these results is still to be investigated, as are many microscopic problems of line mixing and departure from ideality.

\subsubsection{Radiative Rosseland mean opacities}

The calculation of a Rosseland opacity table for substellar objects is a difficult task, and indeed no such table spanning the range of giant planets to M-dwarfs is yet available. An opacity table 

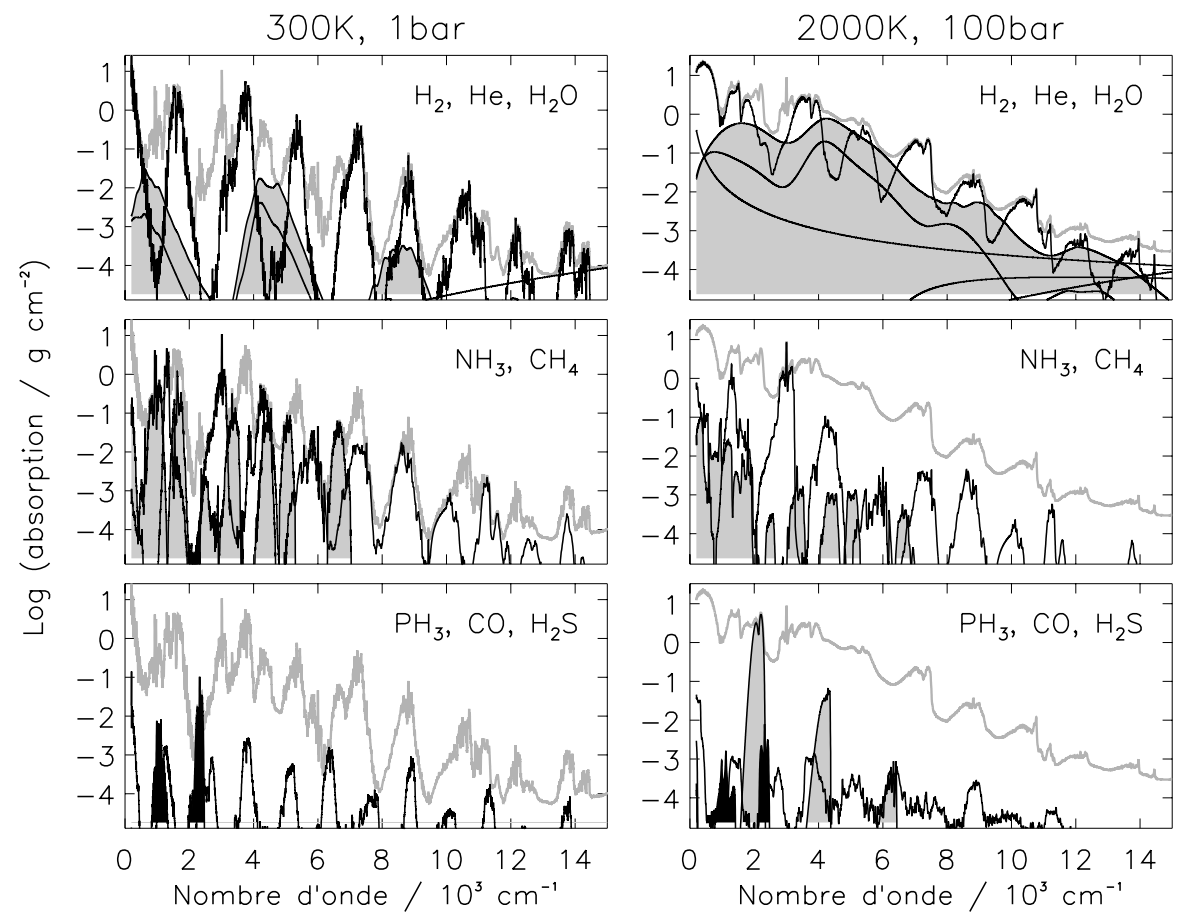

Figure 4.1: Absorption of a solar-composition mixture, at $300 \mathrm{~K}$ and 1 bar (left), and at $2000 \mathrm{~K}$ and 100 bar. Various contributions are shown. Top diagrams: $\mathrm{H}_{2}-\mathrm{H}_{2} \mathrm{CIA}$ (shaded) and $\mathrm{H}_{2} \mathrm{O}$ (grey lines); Middle: $\mathrm{NH}_{3}$ (shaded) and $\mathrm{CH}_{4}$ (grey lines); Bottom: $\mathrm{PH}_{3}$ (shaded, left diagram), $\mathrm{CO}$ (shaded, right diagram) and $\mathrm{H}_{2} \mathrm{~S}$ (grey).

for stars have been calculated by Alexander \& Ferguson (1994), but at low temperature it is dominated by the presence of interstellar-sized grains. It is then essentially designed to the study of circumstellar disks. Another effort was led by Lenzuni et al. (1991) with their zero-metallicity opacity table. As suggested by the name, the calculations includes only hydrogen and helium and its applicability to real giant planets and brown dwarfs is thus limited. The theoretical spectra of M-dwarfs and cooler objects are now relatively good but unfortunately no Rosseland opacity table has been published by modelers. Finally, a limited Rosseland table was computed for Jupiter and Saturn by Guillot et al. (1994a) and Guillot (1999) but it does not include high-temperature species (such as $\mathrm{TiO}$ ), and, as other tables calculated so far, it does not account for the absorption of alkali metals.

I won't attempt to discuss any specifics of these calculations. However the broad features can be understood by looking at the monochromatic absorptions shown in Fig. 4.1. At low temperatures and pressure, the spectrum is dominated by water and methane with a small contribution of $\mathrm{H}_{2}-\mathrm{H}_{2}$ collision-induced absorption, ammonia and phosphine $\left(\mathrm{PH}_{3}\right)$. The molecular bands are relatively narrow. At higher temperatures and pressures, the absorption bands become much broader. The $\mathrm{H}_{2}-\mathrm{H}_{2}$ CIA becomes more important but water still dominates the absorption spectrum. However, methane has almost disappeared in the favor of carbon monoxide, which peaks at $5 \mu \mathrm{m}$. The 
behavior of the Rosseland opacity over this range of conditions evolves mostly because of the displacement of the weighting function $d B_{\nu} / d T$ in eq. (4.4). At $300 \mathrm{~K}$, it is maximum at $830 \mathrm{~cm}^{-1}$, in a spectral region where the absorption is large. At $2000 \mathrm{~K}$, its peak is around $5600 \mathrm{~cm}^{-1}$, and the contribution of the low-absorption region around $1 \mu \mathrm{m}\left(10,000 \mathrm{~cm}^{-1}\right)$ becomes important. At still higher temperatures, the increased abundance of electrons imply a very rapidly increasing $\mathrm{H}^{-}$ and $\mathrm{H}_{2}^{-}$continuous absorption: the Rosseland mean opacity then rises so much that any radiative process becomes very inefficient until eventually conduction dominates.

Two important points are to be mentioned: first, this local minimum of the Rosseland opacity at temperatures of the order of 1500 to $2000 \mathrm{~K}$ which prevails for a zero-metallicity gas is conserved in the presence of water, methane, ammonia, silane and a variety of other species observed in the atmospheres of Jupiter and Saturn. This is due to the fact that these elements all have low absorptions around $1 \mu \mathrm{m}$. The presence of other chemical elements can alter this conclusion. First, those that have low ionization potentials can increase the number of electrons. This is the case of $\mathrm{Al}, \mathrm{Na}$...etc., but has been shown not to be sufficient to erase this minimum (Guillot et al. 1994a). Second, alkali metals have been shown to absorb precisely at these wavelengths (Burrows et al. 2000a) and can therefore greatly affect this conclusion. Finally, the presence of grains/dust/cloud particles can have a very significant effect.

\subsubsection{Clouds and dust}

A great variety of chemical species in condensed form have been identified in the interstellar medium, and their contribution to the energy balance of interstellar clouds and circumstellar disks has been shown to be absolutely essential. Solid grains have also been shown to affect the structure and evolution of red giant stars, and in particular to be determinant for understanding the violent mass loss processes that these objects undergo. Finally, we owe our very existence to the presence of condensed species in our own atmosphere: the presence of clouds, of rain, proves that these phenomena greatly affect the energy balance in Earth atmosphere. This is also the case in the giant planets, and the presence of big particles (i.e. clouds) (generally of unknown composition) is required for a proper fit of the observed spectra.

Condensed grains have such a fundamental importance because of their ability to absorb light: Taken alone, the grains that can potentially condense out of a solar-composition mixture are capable of providing a Rosseland opacity up to $\sim 10 \mathrm{~cm}^{2} \mathrm{~g}^{-1}$ (e.g. Pollack et al. 1985, 1994). This value depends on the abundance and composition of the condensed material, and hence mostly on the temperature of the mixture, but also on the size distribution.

One can define three regimes, depending on the ratio of the wavelength to the size of the grains. For grains much smaller than the wavelength of the incoming light, the opacity is essentially due to Rayleigh scattering and the Rosseland mean is independent of the size. For large grains, the cross section decreases as the grains are bigger (the total mass of condensed material being held constant), and the Rosseland opacity is consequently inversely proportional to the size of the grains. The opacity is maximal at wavelengths of the order of the size of the grain.

Astrophysical opacity tables have generally been calculated assuming a full chemical equilibrium in which the condensing species have been retained. Furthermore, their size distribution has generally been taken to be that of the interstellar medium. A good example is the widely used table provided by Alexander \& Ferguson (1994), which shows several absorption jumps due to the condensation of various species, in particular silicates at temperatures lower than $\sim 2000 \mathrm{~K}$.

However, it is not clear that this approach is even useful in the case of giant planet and brown dwarfs atmospheres. The size distribution then obtained strongly depends on complex advective 
processes and has in most cases nothing to do with that of interstellar clouds. Gravity is indeed a very important factor in planetary atmospheres: it will generally lead to the removal of grains, but several complications can occur due to convection and more generally advection of material. Heterogeneity is also likely to occur, and instabilities can be generated by the presence of clouds. Finally, latent heat release can also be an important factor, as in the case of the Earth, for which cumulus clouds can penetrate the upper atmosphere because of the significant release of energy occurring during the condensation of water vapor to a liquid or solid phase.

The calculation of a mean opacity table for substellar atmospheres that would include condensed species is hence at the least impractical and limited to very special conditions. However, it may be possible to use this simplified treatment by noting that grains often dominate the absorption when they occur. A combination of two tables, one without grains, and one with grains only might be a possibility.

\subsubsection{Conductive opacities}

We have seen that at high temperatures, the number of electrons present yields a very rapid increase of the opacity. Because we are considering environments in which the electrons become partially degenerate, conduction by these electrons can become, at high pressures, an efficient way to transport the internal heat.

In environments in which heat is entirely transported by conduction, the heat flux obeys a standard diffusion equation:

$$
\mathbf{Q}=-K_{\mathrm{c}} \nabla T
$$

where $K_{\mathrm{c}}$ is the thermometric conductivity, expressed in units of $\mathrm{erg} \mathrm{s}^{-1} \mathrm{~cm}^{-1} \mathrm{~K}^{-1}$. An order of magnitude estimate of this quantity for the jovian interior is provided by Stevenson \& Salpeter (1977a):

$$
K_{\mathrm{c}} \approx 10^{8} \rho^{4 / 3} \mathrm{erg} \mathrm{s}^{-1} \mathrm{~cm}^{-1} \mathrm{~K}^{-1}
$$

and $\rho$ is expressed in $\mathrm{g} \mathrm{cm}^{-3}$.

The relationship between the conductive opacity and the thermometric conductivity is

$$
\kappa_{\mathrm{c}}=\frac{4 a c T^{3}}{3 K_{\mathrm{c}} \rho} .
$$

The diffusion equations for radiation and conduction being additive, one can define a conductive + radiative opacity $\kappa$ as

$$
\kappa^{-1}=\kappa_{\mathrm{R}}^{-1}+\kappa_{\mathrm{c}}^{-1}
$$

Tables of either the thermometric conductivity or the conductive opacity have been calculated by Hubbard and Lampe (1969) and more recently by Potekhin et al. (1999). The results by Potekhin et al. indicate slightly smaller opacities by $\approx 10 \%$. Typically, the conductive opacity of the hydrogen gas at $10^{7} \mathrm{~K}$ decreases from about $10^{5} \mathrm{~cm}^{2} \mathrm{~g}^{-1}$ at $\rho=1 \mathrm{~g} \mathrm{~cm}^{-3}$ to $10^{3} \mathrm{~cm}^{2} \mathrm{~g}^{-1}$ for $\rho=100 \mathrm{~g} \mathrm{~cm}^{-3}$.

\subsection{Heat transport}

We have described two ways of transporting heat: radiation and conduction. In the diffusion approximation, i.e. at levels where the medium can be considered isotropic, these fundamental physical processes can be described by relatively simple equations. However, another extremely 
important mechanism has been left out so far: the advection of heat by macroscopic motions. There are many ways to generate heat advection, or convection, and it can take many forms. We will only mention the most simple one: when convection is generated by a destabilizing temperature gradient, and the medium can be considered barotropic (surfaces of constant $P$ and $\rho$ coincide). The method pioneered by Prandtl, Schwarzschild and Ledoux and widely used in stellar physics is explained thoroughly in many textbooks. We will therefore only sketch it.

\subsubsection{Convective instability criterion}

In stellar (or in this case substellar) physics, viscosity is considered negligible and convection is predicted to occur whenever it is energetically favorable. This is unlike e.g. the Rayleigh-Bénard instability for which the system has to overcome a barrier of potential to occur. In stars and giant planets, the barrier is so small that it can be neglected (see later 4.3.3).

In our case, convection is supposed to occur whenever the medium is locally unstable to convection, i.e. when a parcel of fluid displaced upward (resp. downward) is lighter (resp. heavier) than its surrounding. Let us consider this parcel of fluid versus its environment. When it is arbitrarily displaced radially by $\Delta r$, its density changes by $\Delta \rho^{\star}$ and has changed by $\Delta \rho$ in the unperturbed environment. A convective instability then develops if:

$$
\frac{\Delta \rho^{\star}}{\Delta r}<\frac{\Delta \rho}{\Delta r}
$$

Because pressure variations are equilibriated much faster (at the speed of sound) than temperature variations in the interior, this is equivalent to ${ }^{4}$ :

$$
\left(\frac{\partial \rho}{\partial T}\right)_{P, \mu}\left(\frac{d T}{d P}\right)^{\star} \frac{d P}{d r}<\left(\frac{\partial \rho}{\partial T}\right)_{P, \mu}\left(\frac{d T}{d P}\right) \frac{d P}{d r}+\left(\frac{\partial \rho}{\partial \mu}\right)_{T, P}\left(\frac{d \mu}{d P}\right) \frac{d P}{d r},
$$

where $(d T / d P)^{\star}$ corresponds to the temperature variation in the perturbed fluid parcel. We have implicitly considered that the molecular diffusivity is slower than the thermal one so that the mean molecular weight $\mu$ is held constant in the parcel and varies in the environment. Clearly this is not the case for fast chemical reactions as ionization. In that case, convection can be thought to occur in a homogeneous medium.

This implies that convection should develop whenever

$$
\nabla_{T}>\nabla_{T}^{\star}+\frac{\varphi}{\delta} \nabla_{\mu}
$$

where $\varphi$ and $\delta$ are thermodynamical derivatives of the density (equal to 1 for a perfect gas):

$$
\delta=-\left(\frac{\partial \ln \rho}{\partial \ln T}\right)_{P, \mu} ; \quad \varphi=-\left(\frac{\partial \ln \rho}{\partial \ln \mu}\right)_{P, T}
$$

This criterion is not yet in a useful form because neither $\nabla_{T}^{\star}$ nor $\nabla_{T}$ are known a priori. However, one should note that the following inequalities should be satisfied in a convective zone:

$$
\nabla_{\mathrm{rad}}>\nabla_{T}>\nabla_{T}^{\star}>\nabla_{\mathrm{ad}}
$$

\footnotetext{
${ }^{4}$ For simplicity, we forget the time derivative and use $d P / d r$ instead of $\partial P / \partial r$.
} 
where $\nabla_{\text {rad }}$ is the radiative (+conductive) gradient

$$
\nabla_{\mathrm{rad}}=\frac{3}{64 \pi \sigma G} \frac{\kappa P L}{m T^{4}},
$$

$\nabla_{\text {ad }} \equiv(\partial \ln T / \partial \ln P)_{S}$ is the adiabatic gradient, $\nabla_{T} \equiv \partial \ln T / \partial \ln P$ is the real temperature gradient and $\nabla_{T}^{\star}$ is that gradient in the parcel of fluid. The first inequality to the left is due the fact that given a set luminosity, the radiative gradient is a strict maximum to the temperature gradient. The second inequality is a consequence of the convection criterion. The last one is due to the fact that heat can be transported by the parcel only if its motion is slightly superadiabatic i.e. if it looses some of its heat during its ascent.

It is then easy to derive the so-called Schwarzschild-Ledoux criterion for convective instability (Note that $\varphi$ and $\delta$ are positive quantities):

$$
\nabla_{\mathrm{rad}}>\nabla_{\mathrm{ad}}+\frac{\varphi}{\delta} \nabla_{\mu} .
$$

This criterion is fundamental for the evolution of planets to stars. It is important to notice that $\nabla_{\text {rad }}$ is proportional to the luminosity $L$ and to the mean opacity $\kappa$. Convection will occur if $L$ and $\kappa$ are too large so that radiation can transport the heat flux only with a steep, unstable temperature gradient.

\subsubsection{Mixing length theory}

While we now know when convection should occur, we haven't derived an expression for the temperature gradient $\nabla_{T}$. In stellar modeling, this is generally done using the approach due to Prandtl (1925): the Mixing length theory. This is in fact a phenomenological approach and as such it has been widely criticized. In the case of substellar objects, a detailed treatment of convection will generally not be necessary because, as we will see, convection is almost adiabatic.

The main hypothesis of the mixing length theory is that convective elements should dissolve after a "mixing length" $l \equiv \alpha H_{P}$, where $H_{P}$ is the pressure scale height and $\alpha$ is a free parameter of order unity.

We will first assume a homogeneous medium, i.e. $\nabla_{\mu}=0$. By definition, the total flux $F$, radiative flux $F_{\text {rad }}$ and convective flux $F_{\text {conv }}$ obey the following relations:

$$
\left\{\begin{aligned}
F & =F_{\mathrm{rad}}+F_{\mathrm{conv}} \\
F_{\mathrm{rad}} & =\frac{4 a c T^{3}}{\kappa \rho} \frac{T}{H_{P}} \nabla_{T} \\
F & =\frac{4 a c T^{3}}{\kappa \rho} \frac{T}{H_{P}} \nabla_{\mathrm{rad}}
\end{aligned}\right.
$$

Prandtl's approach allows to estimate the convective flux and the convective velocity by integrating the acceleration by the buoyancy force over the mixing length. This is done in several textbooks (e.g. Kippenhahn \& Weigert 1991) and I will not rederive these expressions. After some algebra, one derives the cubical equation of the mixing length:

$$
\left\{\begin{array}{l}
\frac{9}{4} \Gamma^{3}+\Gamma^{2}+\Gamma=A^{2}\left(\nabla_{\mathrm{rad}}-\nabla_{\mathrm{ad}}\right), \\
A=\frac{c_{P} \kappa \rho^{2} \alpha^{2}}{12 \sqrt{2} a c T^{3}}(g \delta)^{1 / 2} H_{P}^{3 / 2},
\end{array}\right.
$$


where $0 \leq \Gamma \leq \infty$ is a parameter characterizing the efficiency of convection, and I used as second parameter of the mixing length a ratio of the volume $V$ of convective elements over their surface $S, V / S=l / 6$. The cubical equation has only one real and positive root: it defines a unique value of $\Gamma$.

The temperature gradient and convective velocity are given by

$$
\begin{aligned}
\nabla_{T} & =\nabla_{\mathrm{ad}}+\frac{\Gamma(\Gamma+1)}{A^{2}}, \\
v & =\frac{\left(g \delta H_{P}\right)^{1 / 2}}{2 \sqrt{2}} \alpha \frac{\Gamma}{A} .
\end{aligned}
$$

The cubical mixing length equation should be consistently solved when calculating the evolution of substellar objects. However, in most cases, convection is found to be very efficient and $\Gamma$ is large. In that case, simpler expressions for the convective velocity and temperature gradient can be obtained:

$$
\begin{aligned}
\nabla_{T}-\nabla_{\mathrm{ad}} & \sim\left[\frac{4 \sqrt{2}}{\alpha^{2} \delta^{1 / 2}} \frac{F_{\mathrm{conv}}}{c_{P} T(\rho P)^{1 / 2}}\right]^{2 / 3}, \\
v & \sim\left[\frac{\alpha \delta}{4} \frac{P}{\rho c_{P} T} \frac{F_{\mathrm{conv}}}{\rho}\right]^{1 / 3} .
\end{aligned}
$$

According to eq. (4.24), the convective flux is $F_{\text {conv }}=F\left(1-\nabla_{T} / \nabla_{\text {rad }}\right)$. When the opacities become large, $\nabla_{\text {rad }} \gg \nabla_{T} \approx \nabla_{\text {ad }}$ and $F_{\text {conv }} \approx F$. Physically, the superadiabatic gradient is an adimensional quantity involving the ratio of the energy per unit mass $F / \sqrt{\rho P}$ to be transported to that of a given layer, $c_{P} T$. The convective velocity is essentially proportional to $(F / \rho)^{1 / 3}$ : since $F$ is a slowly varying function, $v$ should be expected to be larger near the surface, where $\rho$ is smaller. This corresponds to the fact that in a low density material, transporting the same energy requires higher velocities.

Since we consider objects that are almost fully convective, and for which our assumption that $\Gamma \gg 1$ is verified in most of the interior, the value of $\alpha$ can affect the results through the change in the superadiabatic gradient. As we will see next, this value is generally extremely small. The structure of substellar objects is thus weakly dependent on the treatment of convection, except possibly for the largest masses and early in the evolution.

\subsubsection{Properties of convection in substellar objects}

First, let us determine the physical reasons for which the interiors of substellar objects are found to be essentially convective (for direct applications to our giant planets, see Hubbard 1968; Stevenson \& Salpeter 1977b; Guillot et al. 1994b, 1997; Guillot 1999). At any given level, we define a critical opacity $\kappa_{\text {crit }}$ as the Rosseland mean opacity for which $\nabla_{\text {rad }}=\nabla_{\text {ad }}$. It can be seen from the definition of $\nabla_{\text {rad }}$ (Eq. (4.22)) that

$$
\kappa_{\text {crit }}=\frac{16 \nabla_{\mathrm{ad}}}{3} \frac{g}{P}\left(\frac{T}{T_{\mathrm{eff}}}\right)^{4} .
$$

We now assume that $\nabla_{T}$ is approximately constant so that $T \propto P^{\nabla_{T}}$, and that the flux $\sigma T_{\text {eff }}^{4}$ and gravity $g$ are also constant. This yields

$$
\kappa_{\text {crit }} \approx \frac{16 \nabla_{\mathrm{ad}}}{3} \frac{g}{P_{0}}\left(\frac{P}{P_{0}}\right)^{4 \nabla_{T}-1} .
$$


The critical opacity is thus only weakly dependent on the pressure level. Using the definition of the photospheric pressure, and introducing the photospheric opacity $\kappa_{0} \equiv \kappa\left(P_{0}, T_{\text {eff }}\right)$, one finds that

$$
\kappa_{\text {crit }} \approx 8 \nabla_{\mathrm{ad}} \kappa_{0}\left(\frac{P}{P_{0}}\right)^{4 \nabla_{T}-1} .
$$

Note that this expression is relatively simple, but caution should be made regarding to its applicability. We have assumed $g$ and $T_{\text {eff }}$ to be constant, an assumption which generally verified, except in the central regions. The hypothesis $\nabla_{T}=$ cte is more ad hoc. However, because of the small superadiabaticities in substellar objects, $\nabla_{T} \leq \nabla_{\text {ad }}+\epsilon$ where $\epsilon$ is a small quantity (see Eq. (4.33) hereafter). With $\nabla_{\mathrm{ad}} \sim 0.3$, regardless of variations of $\nabla_{T}$, one finds that in most cases $\kappa_{\text {crit }}$ is a function that weakly depends on $P$. We are thus led to the conclusion that substellar objects are mostly convective because of the strong increase of their Rosseland opacities with increasing pressure and increasing temperature.

It should be stressed however that Eq. (4.32) is not valid near the photosphere because it is calculated in the diffusion approximation. One can notice for example that $\kappa_{\text {crit }}$ is a Rosseland mean opacity, whereas $\kappa_{0}$ would correspond more or less to a Planck mean opacity (straight mean weighted by the Planck function).

In the case of isolated substellar objects, we can distinguish two regimes:

1. Cool objects $\left(T_{\text {eff }} \lesssim 1500 \mathrm{~K}\right)$ : The opacity $\kappa$ is essentially due to molecular absorption which is weakly dependent on pressure and temperature. However, an important contribution is due to the $\mathrm{H}_{2}-\mathrm{H}_{2}$ collision-induced absorption which is proportional to $P$. The increase in this opacity guaranties convection.

2. Hot objects $\left(T_{\text {eff }} \gtrsim 1500 \mathrm{~K}\right)$ : The increase in temperature provides a growing number of electrons which greatly contribute to the total opacity via $\mathrm{H}_{2}^{-}$and $\mathrm{H}^{-}$absorption. Convection is then also guaranteed in this regime.

Note that this is the case only for isolated, or weakly irradiated planets or brown dwarfs. The case of strongly irradiated objects will be discussed afterward. Furthermore, any decrease of the Rosseland opacity, such as that due to minimum 1 micron absorption in the absence of alkali metals can yield a small but important radiative region. Finally, the presence of moist convection or of gradients of composition can alter this conclusion. However, this only affects limited regions, and one can consider that substellar objects are mostly convective (however see Chabrier et al. 2000b).

Let us characterize convection in these objects. Under typical conditions, Eq. (4.28) can be shown to yield:

$$
\nabla_{T}-\nabla_{\text {ad }} \approx 10^{-3}\left(\frac{T_{\text {eff }}}{1000 \mathrm{~K}}\right)^{7 / 3}\left(\frac{P}{1 \text { bar }}\right)^{-2 / 3} .
$$

Convection can thus be considered adiabatic in most cases. This is due to the fact that the energy to be transported is relatively small when compared to that available from thermonuclear reactions in stars. Because of this property, the structure and evolution is found to be relatively insensitive to the treatment of convection (and e.g. to the choice of the mixing length parameter $\alpha$ ).

The convective velocity is estimated from Eq. (4.29):

$$
v \approx 150\left(\frac{T_{\text {eff }}}{1000 \mathrm{~K}}\right)^{4 / 3}\left(\frac{\rho}{1 \mathrm{gcm}^{-3}}\right)^{-1 / 3} \mathrm{~cm} \mathrm{~s}^{-1} .
$$

The velocities thus derived are relatively small for giant planets such as Jupiter $\left(T_{\text {eff }} \sim 100 \mathrm{~K}\right)$ and in the interior. They can however reach $100 \mathrm{~m} \mathrm{~s}^{-1}$ at the top of the convective region of the 
hottest brown dwarfs $\left(T_{\text {eff }} \sim 2000 \mathrm{~K}\right)$. Note that in the case of Jupiter, the condensation of water provides another source of energy which is not considered here. Due to that effect, updrafts can reach several 10's of $\mathrm{ms}^{-1}$. (The same principle holds for the Earth's cumulus clouds).

Table 4.1: Properties of convection in Jupiter

\begin{tabular}{rccccc}
\hline \hline & $\begin{array}{c}H_{P} \\
{[\mathrm{~km}]}\end{array}$ & $\begin{array}{c}v_{\text {conv }} \\
{[\mathrm{m} / \mathrm{s}]}\end{array}$ & $\begin{array}{c}\operatorname{Pr}=\nu / \kappa \\
{[]}\end{array}$ & $\begin{array}{c}R e=v d / \nu \\
{[]}\end{array}$ & $\begin{array}{c}R o=v / \omega d \\
{[]}\end{array}$ \\
\hline $\begin{array}{r}\text { Surface } \\
\text { [ }\end{array}$ & 1 & $10^{-4}$ & $10^{9}$ & 1 \\
$\mathrm{PPT} /$ molecular & & & 1 & & \\
Center & 13000 & 0.03 & $10^{-3}$ & $10^{11}$ & $10^{-4}$ \\
\hline
\end{tabular}

Table 4.1 illustrates the properties of convection in Jupiter, based on estimates from Stevenson \& Salpeter (1977a). The pressure scale height varies from a few tens of kilometers near the photosphere to a fraction of the planetary level near the center. The convective velocity is very small deep in the interior, but as discussed, it decreases significantly at smaller densities.

A few adimensional numbers characterize convection itself: the Prandtl number is the ratio between the opacity and the viscosity. It is small in regions where either radiation or convection are relatively efficient at transporting heat (independently of the presence of convection), i.e. near the surface and in the metallic interior where conduction becomes dominant. The Reynolds number compares macroscopic diffusion to the viscosity. It is very large, indicating that convection is turbulent. Finally, the Rossby number is a measure of the importance of rotation on convective motions. It is low, due to the rapid rotation of the planet in $\sim 10$ hours. This indicates that rotation will significantly affect convective motions, implying that convective motions will be mostly confined to a plane perpendicular to the axis of rotation. This gives rise to the so-called Taylor columns (e.g. Busse 1978).

\subsubsection{Possible inhibitions of convection}

A few phenomena are susceptible of inhibiting convection. I will enumerate a few of them:

1. Rotation: In the limit of very low Rossby numbers, convective motions are confined to a plane perpendicular to the axis of rotation (see e.g. Pedlosky 1979). In the case of Jupiter, it has been estimated that this yields a limited increase of the superadiabaticity and is hence negligible to first order (Stevenson 1976a).

2. Magnetic field: In a ionized medium, a strong magnetic field can force motions to follow the magnetic lines. All giant planets in our Solar System possess such a magnetic field. Some low-mass stars have been observed to show variability presumably related to the presence of star spots. This is a strong indication that these stars are magnetic. One can therefore surmise that magnetic dynamos occur in most or all substellar objects. The mechanisms that generate these dynamos have not been fully elucidated, and consequences for convection and heat transport remain unclear.

3. Compositional gradients: The presence of compositional gradients $\left(\nabla_{\mu}>0\right)$ can lead to the inhibition of convection. The problem becomes complex because this gradient is a priori unknown: on one hand, convection tends to homogenize layers, leading to $\nabla_{\mu} \rightarrow 0$; on the 
other hand, sharp interfaces can form for which $\nabla_{\mu} \rightarrow \infty$, yielding sharp, diffusive interfaces. This is indeed observed in the Earth oceans, where salt and heat have opposite effects.

4. Condensation: Phase changes of minor species, such as water can strongly modify convection. First, the latent heat released favors updrafts, as observed in Earth's cumulus clouds. In Jupiter, this leads to convective updrafts of tens of $\mathrm{m} / \mathrm{s}$. However, in hydrogen-helium atmospheres, another effect can be potentially important: in this case, because any condensing species is heavier than the surrounding air, condensation tends to yield a stable compositional gradient $\left(\nabla_{\mu}>0\right)^{5}$. Guillot (1995) shows that convection is locally inhibited when the abundance of the condensing species is larger than a certain critical value. This value is of the order of 5, 15 and 40 times the solar values for $\mathrm{H}_{2} \mathrm{O}, \mathrm{CH}_{4}$ and $\mathrm{NH}_{3}$, respectively. The temperature profiles of Uranus and Neptune retrieved from radio occultation of Voyager 2 indeed show a strong superadiabaticity in the region of methane condensation, implying that convection is probably inhibited by this mechanism (Guillot 1995).

\footnotetext{
${ }^{5}$ This is unlike the Earth's atmosphere in which the condensing molecule, water $(\mu=18)$, is lighter than air $(\mu=29)$.
} 


\section{Chapter 5}

\section{Interior structures of our giant planets: numerical integrations \& results}

\subsection{Basic principles}

Constraints on the interior structure of the giant planets of our Solar System are derived from knowledge of their mass, $M$, equatorial radius $a$, and gravitational moments $J_{2}$ and $J_{4}$. Measurements of these quantities still go back to the Pioneer and Voyager missions.

Basically, the procedure is to integrate the hydrostatic equations including rotation using appropriate equations of state, opacities and a set of observed parameters (mass, surface temperature...etc.). The a priori unknown composition is constrained by the gravitational moments. In the case of Uranus and Neptune, we will see that another approach has been proposed which simply relies on the computation of random density profiles. It is to be stressed that in the absence of other information such as a vibration spectrum, only a few moments of the interior density can be constrained. Most of the knowledge concerning the interiors of these planets is indirect: it heavily relies on the input physics.

All four giant planets appear to emit more energy than they receive from the Sun (see Table 1.3 and section 1). As first proposed by Hubbard (1968) for Jupiter, this implies that their interior is hot, fluid and because of the large opacities (see previous section), mainly convective. This is an essential property which allow to model these objects with the same underlying physics.

I will first discuss the case of Jupiter and Saturn, which are mostly formed with hydrogen and helium. These two planets have been extensively modeled (see Hubbard \& Marley 1989; Zharkov \& Gudkova 1992; Chabrier et al. 1992; Guillot et al. 1994b; Gudkova \& Zharkov 1999), but these works generally aimed at finding a limited sample of models matching the observational constraints. I choose to present models calculated in the purpose of extensively exploring the set of parameters (Guillot 1999a). 
I will then present briefly the cases of Uranus and Neptune. These planets are mostly made of ices, and their interior structure is consequently more difficult to grasp. They are also distantly connected to the much more massive brown dwarfs and extrasolar planets that have been detected thus far. However, they are also a crucial piece in the puzzle to understand how the Solar System was formed.

\subsection{Jupiter and Saturn}

\subsubsection{Input data}

\section{Gravitational field}

The characteristics of Jupiter and Saturn's gravity fields as obtained from spacecrafts measurements are listed in Table 5.1. Note that the rotation rate $\omega$ is that of the planets' magnetic field, assumed to be tied to the rotation of the deep interior.

Table 5.1: Characteristics of the gravitational fields

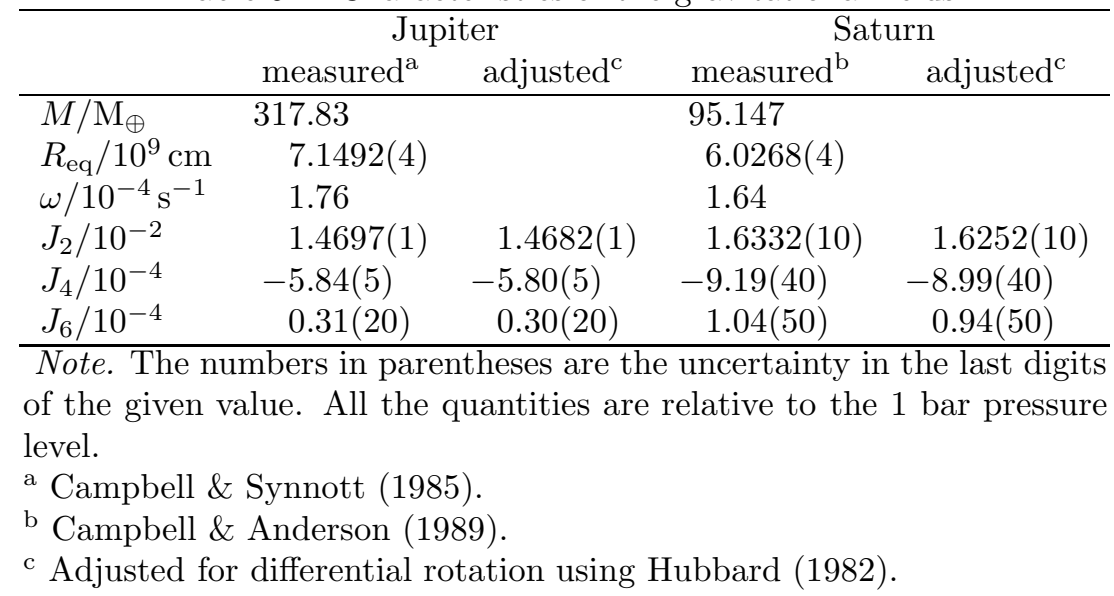

A complication arises from the fact that the equations derived from that theory generally assume the planet to be rotating as a solid body. Observations of the atmospheric winds show significant variations with latitude, however (e.g., Gierasch \& Conrath 1993). The question of the depth to which these differential rotation patterns extend is still open. Hubbard (1982) has proposed a solution to the planetary figure problem in the case of a deep rotation field that possesses cylindrical symmetry. It is thus possible to derive, from interior models assuming solid rotation, the value of the gravitational moments that the planet would have if its surface rotation pattern extended deep into its interior. It is a priori impossible to prefer one model to the other, and I will therefore present calculations assuming both solid and differential rotation. Table 5.1 gives both the measured gravitational moments, and those corrected for differential rotation. 


\section{Atmospheric temperatures}

The temperatures at the tropopause (at pressures of about 0.3 bar) are relatively well constrained by direct inversions of infrared spectra. These predict relatively large latitudinal temperature changes of the order of $10 \mathrm{~K}$ (Conrath et al. 1989). The temperature gradients decrease with tropospheric depth, as interior convection presumably becomes more efficient in redistributing the heat. However, the accuracy of this method drops rapidly with increasing pressure and does not reach levels deep enough to be used as surface condition for interior models. So far, the only reliable measurement of the deep tropospheric temperature of a giant planet is that from the Galileo probe in Jupiter: $166 \mathrm{~K}$ at 1 bar (Seiff et al. 1998). It is not clear however how representative of the whole planet this measurement is. Previous analyses have relied upon (local) radio occultation data acquired with the Pioneer and Voyager spacecrafts (Lindal et al. 1981, 1985) that predicted 1 bar temperatures of $165 \pm 5 \mathrm{~K}$ in Jupiter and $134.8 \pm 5 \mathrm{~K}$ in Saturn. The temperatures inferred from these data are however dependent on the assumed mean molecular weight $\bar{m}$. The Galileo helium mixing ratio, applied to the Voyager data would yield a temperature of $170.4 \mathrm{~K}$ at 1 bar in Jupiter. It is therefore reasonable to assume that the uncertainty on these temperatures if of the order of $\sim 5 \mathrm{~K}$.

\section{Atmospheric abundances}

Because Jupiter and Saturn are believed to be relatively well-mixed, precise measurements of atmospheric abundances is crucial for modeling the interior. First, helium is found in relatively small abundance: Solar evolution models indicate that the protostellar helium mass mixing ratio relative to hydrogen was $Y /(X+Y)=0.270 \pm 0.005$ (Bahcall \& Pinsonneault 1995). In situ measurements of that quantity in Jupiter yield $Y /(X+Y)=0.238 \pm 0.007$ (von Zahn et al. 1998). Combined radio occultation measurements and spectra analysis from Voyager 2 indicate that, in Saturn, $Y /(X+Y)=0.06 \pm 0.05$ (Conrath et al. 1984). This last value has been challenged by several approaches (Guillot 1999; Hubbard et al. 1999; Conrath \& Gautier 2000) and could be significantly larger. However, it still appears to be smaller than the protosolar value.

The conclusion that more helium was present in the protosolar nebula gas from which Jupiter and Saturn formed than is observed today in their atmospheres seems inescapable. As discussed in section 3, this implies the existence of a hydrogen/helium phase separation, in which helium droplets can grow sufficiently fast to be dragged down by gravity despite convection (Salpeter 1973; Stevenson \& Salpeter 1977b). The fact that the Galileo probe measured a depleted abundance of neon is also indicative of such a phase separation, as neon tends to dissolve into the helium-rich drops (Roulston \& Stevenson 1995).

The measured abundances of other elements also provide important clues to the composition of the planets. Both Jupiter and Saturn are globally enriched in heavy elements compared to the Sun. In Jupiter, the in situ measurements of the Galileo probe are compatible with a $\sim 3$ times solar enrichment of carbon, sulfur, argon, xenon and krypton (Niemann et al. 1998; Owen et al. 1999). It is still unclear as to whether nitrogen is close to solar (by a factor 1 to 1.5; de Pater and Massie 1985), moderately (2.2 to 2.4; Carlson et al. 1992) or strongly enriched (3.5 to 4.5 times solar; Folkner et al. 1998). Water is still a problem because of its condensation at deep levels, and only a lower limit of $\sim 0.1$ times solar can be inferred from the measurements. The enrichment in noble gases (except neon) is problematic and bears directly on formation issues. It has been proposed that these elements are brought to the planet in the form of clathrates (Gautier et al. 2001).

Unfortunately, the uncertainties for Saturn are still relatively large. Its atmosphere is enhanced 
in carbon by a factor of 2 to 7 , and in nitrogen by a factor 2 or more (Gautier \& Owen 1989). Observationally, it could therefore be more rich in heavy elements than the jovian atmosphere. This will be tested by the Cassini-Huygens mission.

\section{Equations of state}

Ideally, one should use an equation of state valid for any chemical composition. This is of course unrealistic. The most recent astrophysical equation of state for hydrogen and helium provided by Saumon, Chabrier \& Van Horn (1995) does not account for interactions between the two species. The presence of other species can be added using generally less reliable equations of state.

Figure 5.1 compares different pressure-temperature profiles for Jupiter and Saturn, using the various equations of state described here. The figure is intended to provide an estimate of the uncertainties on the various equations of state (hydrogen-helium, heavy elements). It is important to notice at this point that Saturn's interior lies mostly in a relatively well-known region of the hydrogen-helium EOS, i.e. in which hydrogen is molecular, whereas a significant fraction of Jupiter's interior is at intermediate pressures (one to a few Mbar) for which the EOS is most uncertain.

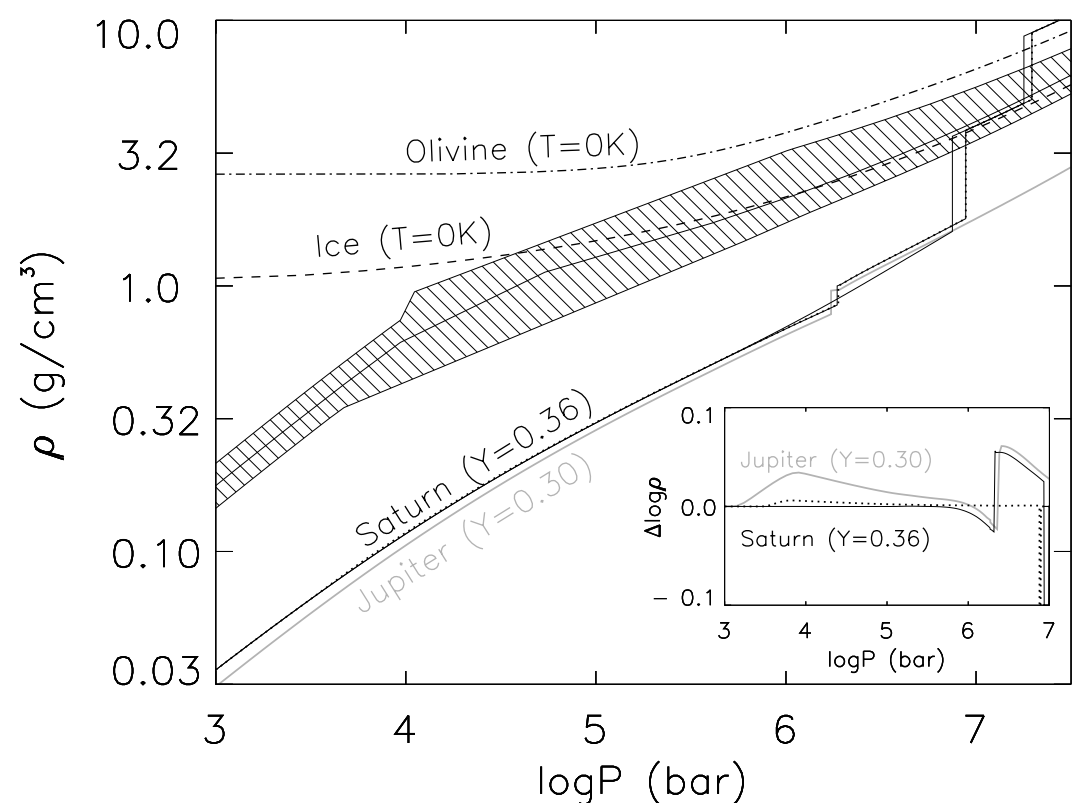

Figure 5.1: Density profiles in models of Jupiter (gray line) and Saturn (continuous lines: adiabatic i-EOS and PPT-EOS models; dashed: non-adiabatic i-EOS model). Upper curves (dashed and dotdashed) are $T=0 \mathrm{~K}$ density profiles for water ice and olivine (from Thompson 1990). The dashed region represents the assumed uncertainty on the EOS for heavy elements $\left(\rho_{Z}(P, T)\right)$. Within this region, the continuous line corresponds to our "preferred" profile for $\rho_{Z}$. Inset: Differences of the decimal logarithm of the Saturn density profiles with the same profile using the $i$-EOS and an adiabatic structure (plain and dotted lines). The gray line corresponds to the same difference but for a PPT-EOS non-adiabatic Jupiter model. (From Guillot 1999a). 


\section{Opacities}

As discussed in section 4 the Rosseland opacities available for models of Jupiter and Saturn are still uncertain. A small table is provided by Guillot (1999a) but does not include the absorption due to alkali metals. It therefore predicts the existence of a radiative region in both Jupiter and Saturn at temperatures around $1500 \mathrm{~K}$ and pressures of $1-10 \mathrm{kbar}$. However, including the absorption of sodium and potassium, as observed in brown dwarfs (Burrows et al. 2000a) provides the required opacity source and the radiative regions then disappear for both planets (Freedman et al. in preparation). The models presented hereafter include uncertainties on the opacities as follow: a minimum value is set by the calculation of a Rosseland opacity table assuming no alkali metals, and thus include the presence of a radiative zone. Other models simply assume that the planets are fully convective. The differences between these models are in fact, in term of interior structure, relatively limited. However, we will see that the presence of a radiative zone affects the evolution more significantly.

\subsubsection{Construction of models}

Most models of Jupiter and Saturn assume a three-layer structure: a helium-poor molecular region, a helium-rich metallic region and a central dense core. The fact that the molecular/metallic transition coincides with a jump in the abundance of helium is related to the idea that helium is most insoluble in low-pressure metallic hydrogen, as obtained from calculations assuming full ionization (see section 3). The consequences of a different phase diagram have not been calculated so far.

The three regions are linked to three parameters: $M_{\text {core }}$ the mass of the core, $Z_{\text {mol }}$ and $Z_{\text {met }}$ the mass mixing ratio of heavy elements in the molecular and metallic envelopes, respectively. (The helium mixing ratio in the molecular envelope is set equal to the atmospheric value; That in the metallic region is constrained by the fact that the total helium/hydrogen ratio should be equal to the protosolar value).

The total mass of the planet being fixed, the observational constraints are the equatorial radius $R_{\text {eq }}$ and gravitational moments $J_{2}, J_{4}$ and $J_{6}$, measured with respective observational uncertainties $\sigma_{R_{\text {eq }}}, \sigma_{J_{2}}, \sigma_{J_{4}}$ and $\sigma_{J_{6}}$. In the framework of the three-layer models, the adjustable parameters are $Z_{\text {mol }}, Z_{\text {met }}$ and $M_{\text {core }}$. A way of finding models matching the observational constraints is therefore to minimize the following function:

$$
\chi^{2}\left(Z_{\mathrm{mol}}, Z_{\mathrm{met}}, M_{\text {core }}\right)=\frac{1}{4}\left[\left(\frac{\Delta R_{\mathrm{eq}}}{\sigma_{R_{\mathrm{eq}}}}\right)^{2}+\left(\frac{\Delta J_{2}}{\sigma_{J_{2}}}\right)^{2}+\left(\frac{\Delta J_{4}}{\sigma_{J_{4}}}\right)^{2}+\left(\frac{\Delta J_{6}}{\sigma_{J_{6}}}\right)^{2}\right],
$$

where $\Delta R_{\text {eq }}, \Delta J_{2}, \Delta J_{4}, \Delta J_{6}$ are the differences between observed and theoretical $R_{\text {eq }}, J_{2}, J_{4}$ and $J_{6}$. The non-uniqueness of solutions matching the observed gravitational fields is found to be mostly due the uncertainty on $J_{4}$. So far, no useful constraint can be derived from the values of $J_{6}$, owing to their large observational uncertainties (see Table 5.1). 


\subsubsection{Results}

The resulting interior models of Jupiter matching all available observations are shown in fig. 5.2. Hundreds of models have been calculated, but the solution is represented as a filled area instead as dots for an easier interpretation of the figure. A striking result obtained from Jupiter's modeling is the large uncertainty due to our relatively poor knowledge of the behavior of hydrogen at Mbar pressures. As a consequence, two kind of solutions are found depending on one using the SaumonChabrier PPT EOS, or the one that is smoothly interpolated between the molecular and the metallic fluids. The uncertainties in the solutions are not due to the qualitative difference at the molecular/metallic transition but instead by the quantitatively different density profiles, as seen in fig. 5.1. Any solution between the two regions in fig. 5.2 would be valid, provided the "true" EOS for hydrogen lies between the PPT and interpolated EOSs.

More quantitatively, fig. 5.2 shows that an upper limit to Jupiter's core mass is rather small, i.e. about $10 \mathrm{M}_{\oplus}$ only. This is significantly smaller than found $\sim 20$ years ago, the main difference being due to the improved EOSs. The lower limit on the core mass is found to be zero: in this case, Jupiter could have no core, or a very small one. This corresponds however to rather extreme models, assuming a hydrogen EOS close to the interpolated one, and a large $J_{4}$ value. The lower panel of fig. 5.2 also indicates that this corresponds to a planet that is enhanced in heavy elements by 4 to 6 times over the solar value (assuming $Z_{\text {mol }}=Z_{\text {met }}$, a consequence of the presence of no physical discontinuity of the EOS). Generally, it is found that Jupiter's molecular region is enriched in heavy elements by 1.5 to 6.5 times the solar value, in agreement with the observations that indicate $\mathrm{a} \sim 3$ times solar enrichment for $\mathrm{C}, \mathrm{N}, \mathrm{S}$.

In the case of Saturn (fig. 5.3), the solutions depend less on the hydrogen EOS because the Mbar pressure region is comparatively smaller. The total amount of heavy elements present in the planet can therefore be estimated with a better accuracy than for Jupiter. It is interesting to see that presently, we do not know which of Jupiter and Saturn contain more heavy elements in absolute value! However, because Saturn's metallic region is deeper into the planet, it mimics the effect that a central core would have on $J_{2}$. The uncertainty on $M_{\text {core }}$ is therefore large. In fig. 5.3 constraints obtained from the evolutionary models have been used to eliminate models that otherwise satisfied the static constraints (see Guillot 1999a for details). Saturn's core is therefore found to be between 6 and $17 \mathrm{M}_{\oplus}$. Saturn's enrichment in heavy elements is found to be generally larger than in the case of Jupiter, but with a considerable uncertainty in the metallic region.

Figures 5.2 and 5.3 also show as arrows the significance of various sources of uncertainties for estimating precisely the parameters of the interior structure. The uncertainty on the measured value of $J_{4}$ is shown to significantly affect the results: in the case of Saturn in particular, a more accurate measurement (that the Cassini-Huygens mission will probably provide) is expected to substantially narrow the ensemble of viable models. Note that in the case of Saturn, a more accurate determination of the surface temperature and of the helium to hydrogen ratio would also be invaluable for better constraining the interior models. 

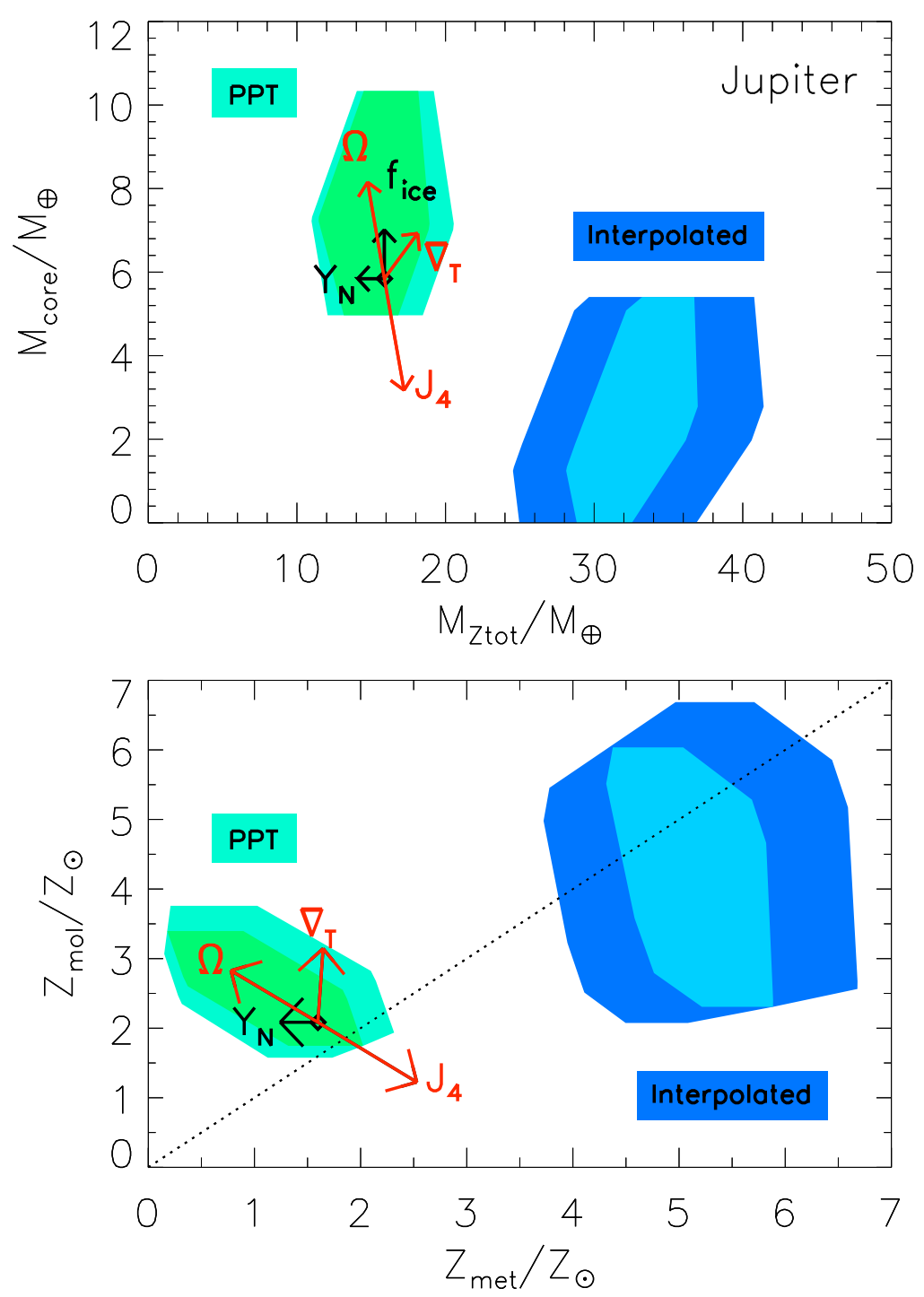

Figure 5.2: Constraints on Jupiter's interior structure. The upper panel shows values of the core mass $\left(M_{\text {core }}\right)$ and total mass of heavy elements $\left(M_{Z \text { tot }}\right)$ of models matching all available observational constraints. The lower panel shows the mass mixing ratio of heavy elements of the molecular $\left(Z_{\mathrm{mol}}\right)$ and metallic $\left(Z_{\mathrm{met}}\right)$ regions, in solar units $\left(Z_{\odot}=0.0192\right)$. The two different regions correspond to different EOSs for hydrogen (see text). Arrows indicate the direction and magnitude of the assumed uncertainties, if $J_{4}$ or $Y_{\text {proto }}$ are increased by $1 \sigma$, rotation is assumed to be solid (" $\Omega$ "), the core is assumed to be composed of ices only (" $f_{\text {ice" }}$ ) and if Jupiter's interior becomes fully adiabatic ( $\nabla_{T}$ "). The dashed line in the lower panel indicates a homogeneous abundance of heavy elements $\left(Z_{\mathrm{mol}}=Z_{\mathrm{met}}\right)$. [Adapted from Guillot 1999a]. 

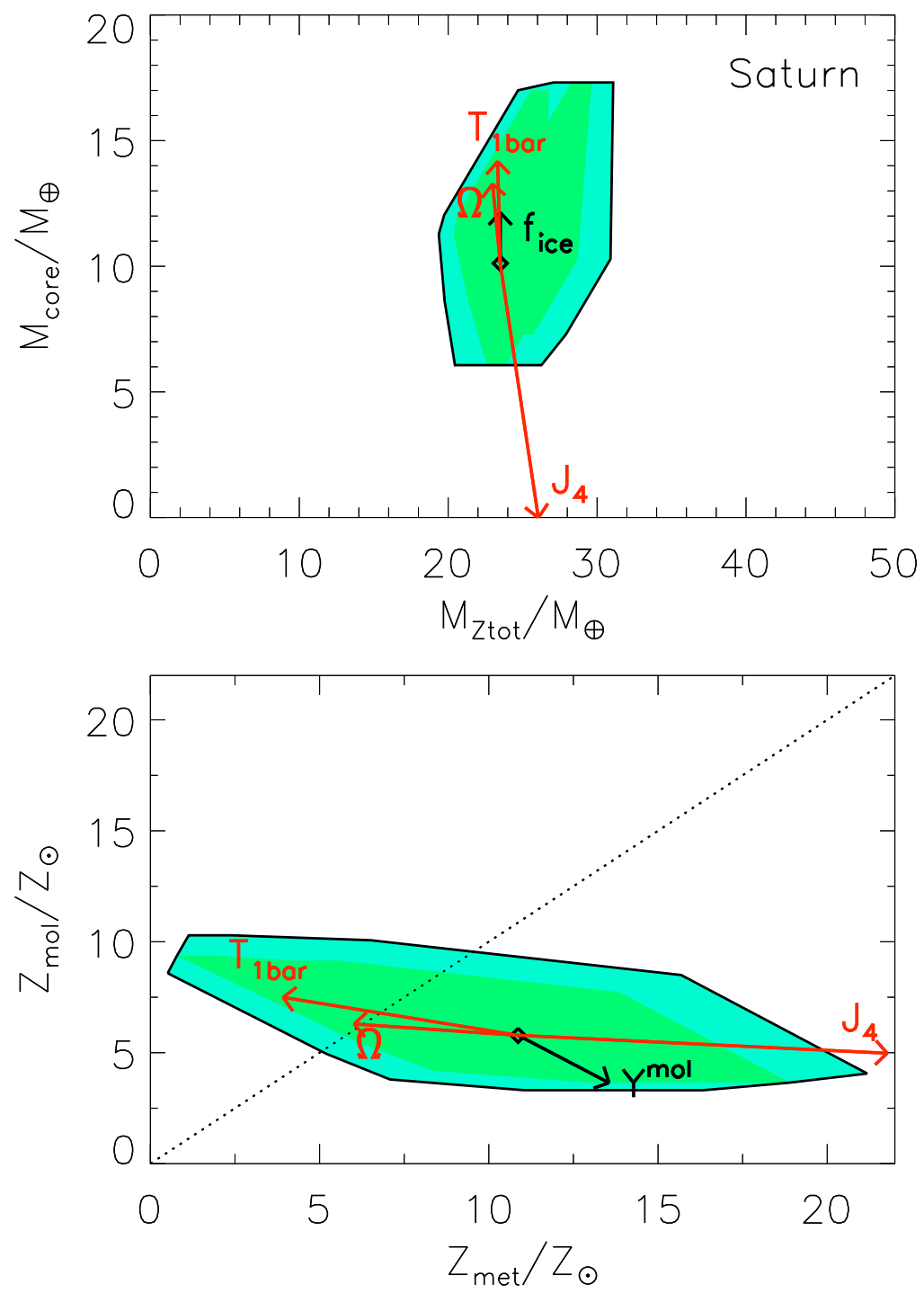

Figure 5.3: Same as fig. 5.2 in the case of Saturn. The solutions for the PPT and interpolated hydrogen EOSs are very similar and are thus not highlighted. The arrow labeled $T_{1}$ bar corresponds to an increase of Saturn's 1 bar temperature from 135 to $145 \mathrm{~K}$. The arrow labeled $Y^{\mathrm{mol}}$ corresponds to an increase of the helium mass mixing ratio from 0.16 to 0.21 . [Adapted from Guillot 1999a]. 


\subsection{Uranus and Neptune}

Spectroscopic measurements indicate that their hydrogen-helium atmospheres contain a large proportion of heavy elements, mainly $\mathrm{CH}_{4}$, which is enriched by a factor $\sim 30$ compared to solar composition (see table 1.2). The two planets have similar masses $\left(14.53 \mathrm{M}_{\oplus}\right.$ for Uranus, $17.14 \mathrm{M}_{\oplus}$ for Neptune) and radii. Neptune's larger mean density is partly due to greater compression, but could also be the result of a slightly different composition. The gravitational moments impose that the density profiles lie close to that of "ices" (a mixture initially composed of $\mathrm{H}_{2} \mathrm{O}, \mathrm{CH}_{4}$ and $\mathrm{NH}_{3}$, but whose composition most probably does not consist of intact molecules in the planetary interior), except in the outermost layers, which have a density closer to that of hydrogen and helium (Marley et al. 1995; Podolak et al. 2000). Three-layer models of Uranus and Neptune consisting of a central "rocks" core (magnesium-silicate and iron material), an ice layer and a hydrogen-helium gas envelope have been calculated (Podolak et al. 1991; Hubbard et al. 1995).

The fact that models of Uranus assuming homogeneity of each layer and adiabatic temperature profiles fail in reproducing its gravitational moments seem to imply that substantial parts of the planetary interior are not homogeneously mixed (Podolak et al. 1995). This could explain the fact that Uranus' heat flux is so small: its heat would not be allowed to escape to space by convection, but through a much slower diffusive process in the regions of high molecular weight gradient. Such regions would also be present in Neptune, but much deeper, thus allowing more heat to be transported outward. The existence of these non-homogeneous, partially mixed regions are further confirmed by the fact that if hydrogen is supposed to be confined solely to the hydrogen-helium envelope, models predict ice/rock ratios of the order of 10 or more, much larger than the protosolar value of $\sim 2.5$. On the other hand, if we impose the constraint that the ice/rock ratio is protosolar, the overall composition of both Uranus and Neptune is, by mass, about $25 \%$ rocks, $60-70 \%$ ices, and $5-15 \%$ hydrogen and helium (Podolak et al. 1991, 1995; Hubbard et al. 1995). An upper limit to the total amount of hydrogen and helium present in these planets is $3 \mathrm{M}_{\oplus}$ for Uranus and $5 \mathrm{M}_{\oplus}$ for Neptune (Podolak et al. 2000).

The characteristics of typical models of the four giant planets are summarized in fig. 5.4, including corresponding uncertainties in the temperature profiles. The distinction between the "gas giants" Jupiter and Saturn and the smaller "ice giants" Uranus and Neptune is evident. 


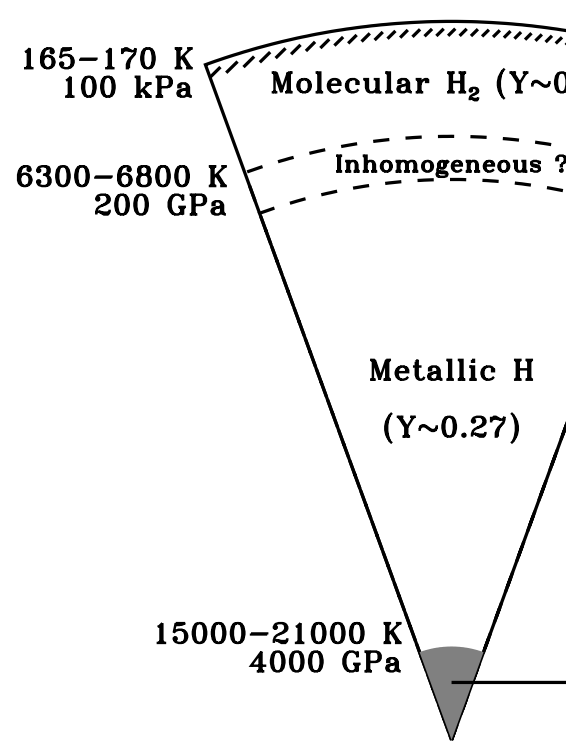

Jupiter

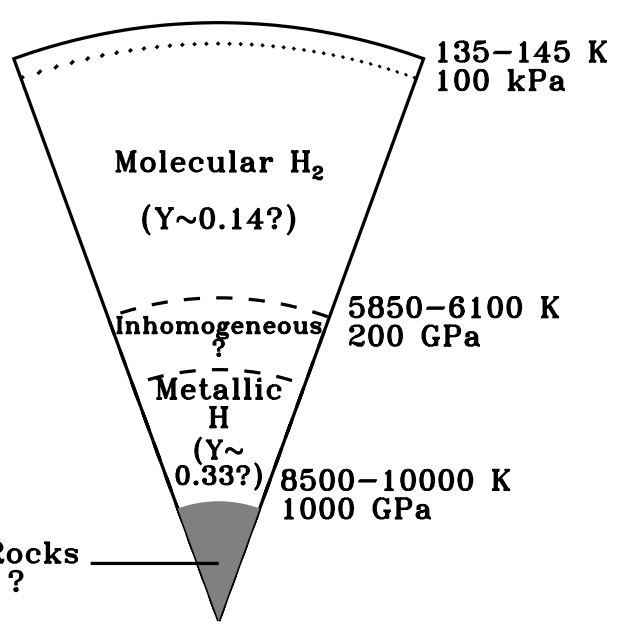

Saturn

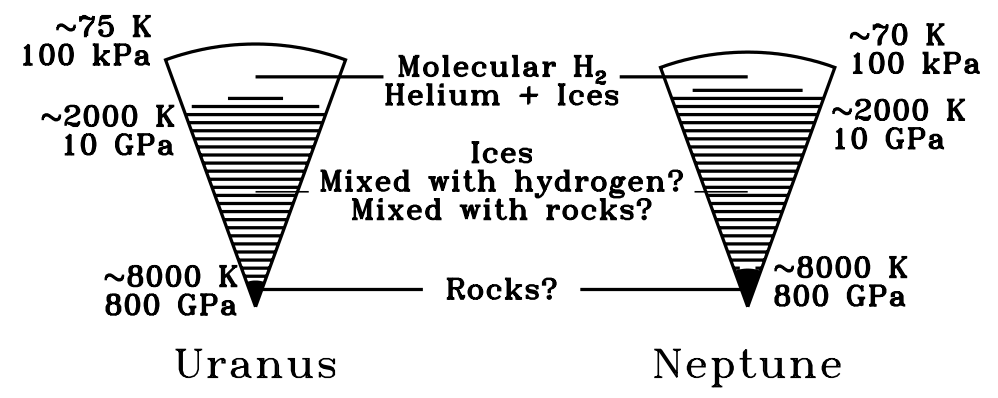

Figure 5.4: Schematic representation of the interiors of Jupiter, Saturn, Uranus and Neptune. The hashed region indicate a possible radiative zone (in Jupiter, it corresponds to $P \sim 0.15$ to $0.6 \mathrm{GPa}$, $T \sim 1450$ to $1900 \mathrm{~K}$, and $R \sim 0.990$ to $0.984 \mathrm{R}_{\mathrm{J}}$; in Saturn, it is located around $P \sim 0.5 \mathrm{GPa}$, $\left.T \sim 1700 \mathrm{~K}, R \sim 0.965 \mathrm{R}_{\mathrm{S}}\right)$. The radiative zone are expected to disappear in the presence of alkali metals. The range of temperatures for Jupiter and Saturn is for models neglecting the presence of the inhomogeneous region. Helium mass mixing ratios $Y$ are indicated. In the case of Saturn, it is assumed that $Y /(X+Y)=0.16$ in the molecular region. The size of the central rock and ice cores of Jupiter and Saturn is very uncertain. Two representative models of Uranus and Neptune are shown, but their actual interior structure may be significantly different (see text). [From Guillot $1999 \mathrm{~b}]$. 


\subsection{Consequences for formation models}

\subsubsection{The minimum mass solar nebula}

The composition of the giant planets provides crucial information to understand the formation of planets in general. A useful first indication of the structure of the early protosolar nebula comes from the estimation of the minimum amount of gas that initially had to be present in the disk in order to form the planets that we see today. The result is commonly called the minimum mass nebula (see Weidenschilling 1977; Hayashi 1981). Fig. 5.5 shows the minimum surface density $\left(\mathrm{g} / \mathrm{cm}^{2}\right.$ projected onto the plane of the Solar System) of hydrogen and helium, as a function of the distance to the Sun, assuming that the planets were formed at their present locations, and using the most recent interior models for Jupiter, Saturn, Uranus and Neptune. The gas to solids ratio was assumed to lie between 55 and 90; these two extremes correspond to (1) the condensation of all species except $\mathrm{H}, \mathrm{He}$, and noble gases, and (2) the condensation of only water and metals and only small amounts of condensed $\mathrm{C}$ (which is then assumed to mostly remains in the form the gaseous $\mathrm{CO}$ ) and $\mathrm{N}$ (remaining mostly bound up as $\mathrm{N}_{2}$ ), respectively.

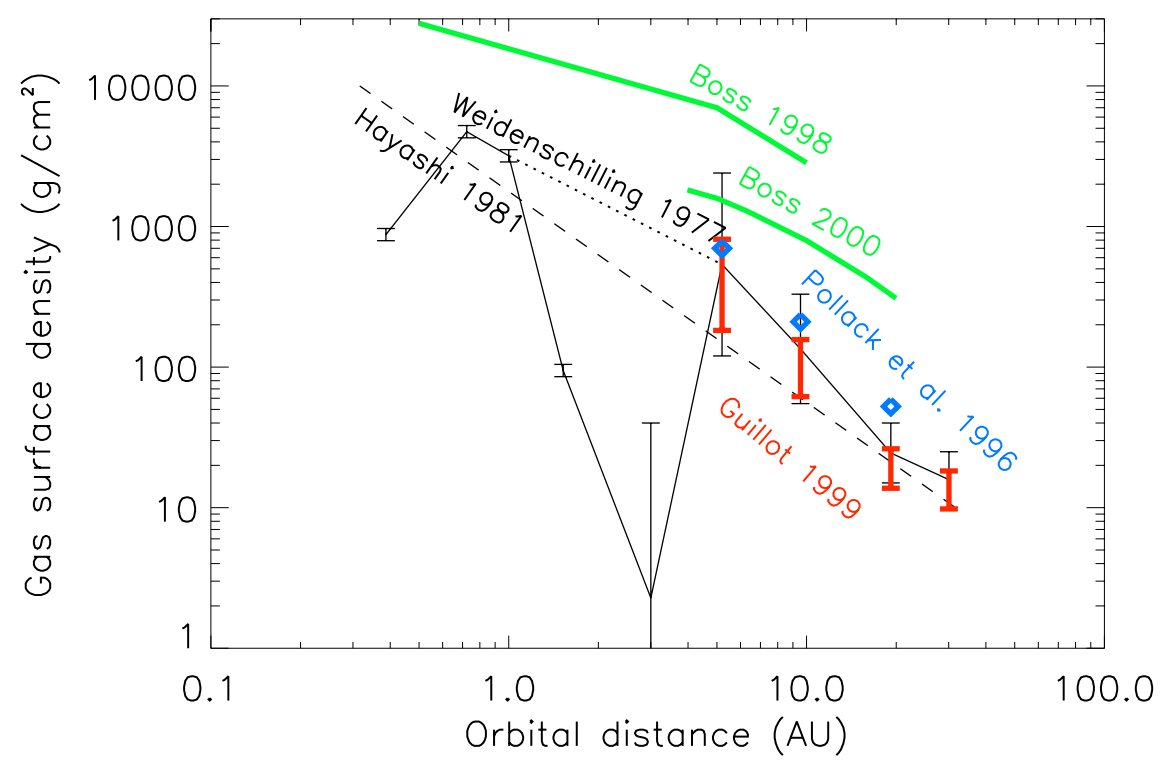

Figure 5.5: Surface density of hydrogen and helium as a function of distance to the Sun, as estimated by various workers. The dashed and plain black lines correspond to the minimum mass protosolar nebula as derived by Hayashi (1981) and Weidenschilling (1977) respectively. The thicker vertical error bars outside $5 \mathrm{AU}$ are updates of the Weidenshilling values using interior models of the outer planets from Guillot (1999a). The diamonds are the optimal surface densities for giant planet formation in a core-accretion scenario, assuming a gas to solids ratio of 70 (Pollack et al. 1996). The two upper lines (Boss 1998, 2000) correspond to a scenario of formation of Jupiter and possibly Saturn by direct gravitational instability in the gas. (From Guillot \& Gladman 2000). 
In Fig. 5.5, the surface density required by two models of formation of the giant planets are indicated. The most "standard" model of formation of the giant planets is based on the formation of a solid protoplanetary embryo followed by the capture of the surrounding hydrogen and helium, on a few million year time scale (Pollack et al. 1996). The density required to form Jupiter in less than 10 million years is just slightly over the the new median estimate for the minimum mass nebula. Neglecting any migration process, this implies that between 20 and $75 \%$ of the solids in that region have been lost, probably due to dynamical evolution. (These numbers account for the fact that the gas to solids ratio has to be the same when comparing different results). The density increases over the minimum one required to form Saturn, Uranus and Neptune is higher, implying an even larger ejection efficiency (or other loss mechanism) for solids in those regions.

An alternative model that might explain the formation of Jupiter is by direct gravitational instability of the gas itself, on much shorter time scales (Boss 1998, 2000). This requires even larger densities than in the core-accretion scenario, but it could be advocated that part of the gas present in the disk at that early times has been accreted onto the star. The subsequent formation of central cores in these models would require an early settling of heavy elements.

\subsubsection{Delivering planetesimals to the giant planets}

To study the delivery of heavy-element rich planetesimals to the forming giant planets, Guillot \& Gladman (2000) performed extensive numerical dynamical simulations of the fate of 10,000 massless particles distributed between 4 and 35 AU. In a baseline model, the masses and radii of the giant planets were set to their present-day values, exploring a scenario in which the planets "suddenly" reach nearly their current masses by a rapid gas accretion onto a much smaller core. After $100 \mathrm{Myr}, 61 \%$ of the initial particles had been ejected out of the system, $23 \%$ had been sent to the Oort cloud (aphelia larger than 10,000 AU), with only $13 \%$ remaining in the system. Only $4 \%$ of the particles impacted one of the four giant planets. In this physical scenario, the probability of impact is low compared to that of ejection, mainly due to the presence of Jupiter, to which the other giant planets efficiently 'pass' their planetesimals. However, this inefficiency of planetesimal accretion poses grave problems when we consider the known mass of heavy elements in the giant planets.

Focusing on the core-accretion scenario, fig. 5.6 shows the accretion efficiency, defined as the ratio between the inferred amount of heavy elements in the giant planets (Guillot 1999a) and the amount of solids required for their formation (Pollack et al. 1996). Planets with the present characteristics are found to be too efficient at ejecting material from the system compared to accreting it, as indicated by the diamonds on fig. 5.6.

Guillot \& Gladman (2000) therefore propose that the heavy elements present in the giant planets today were captured first during a runaway growth phase, probably yielding the cores that are observed today and second during an extended phase during which the planets had large effective capture radii $\left(\sim 3 \overline{\mathrm{R}}_{\mathrm{J}}\right)$ but relatively small masses $\left(\sim 20 \mathrm{M}_{\oplus}\right)$. This case corresponds to triangles in fig. 5.6 and agrees with the accretion efficiencies needed for the giant planets' envelopes (lower thick error bars). 


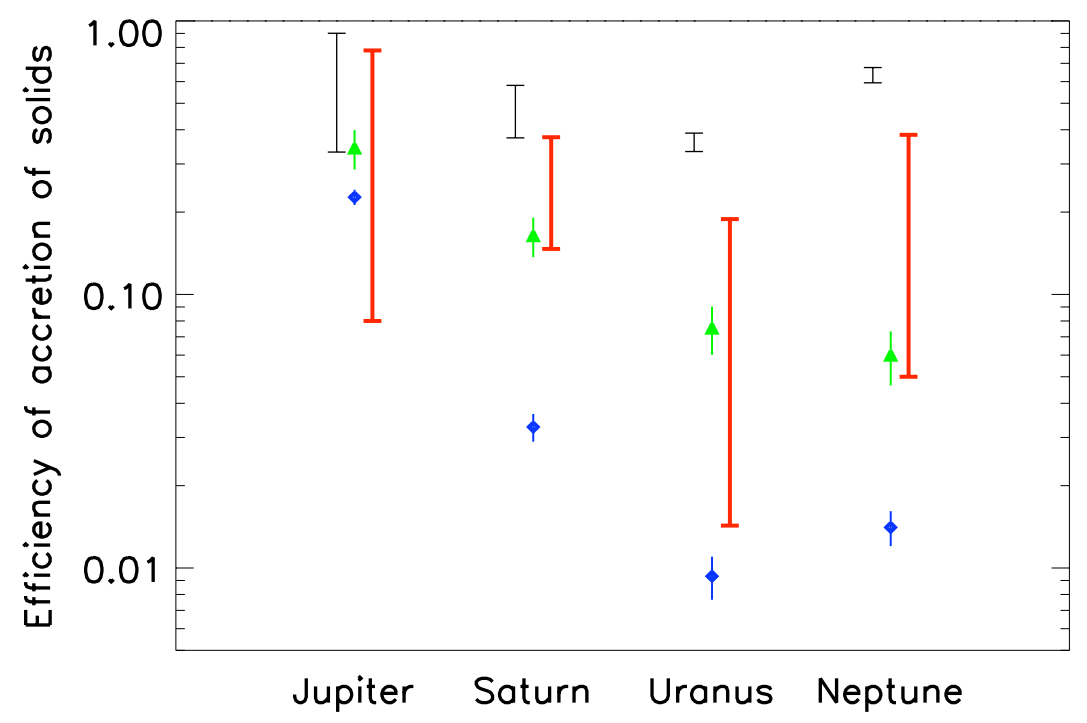

Figure 5.6: Accretion efficiencies required to form the giant planets in the core accretion scenario. The accretion efficiency for a given planet is defined as the quantity of heavy elements that had to impact the planet divided by the total amount of heavy elements within the annulus that extends halfway to the next planet. The upper left error bar for each planets corresponds to the ratio between the total mass of heavy elements and the quantity of solids required by Pollack et al. (1996). The lower thick error bars account for core formation by runaway growth, and include only heavy elements in the envelopes. Diamonds are accretion probabilities in the Jupiter, Saturn, Uranus and Neptune regions, respectively, as calculated in the standard case. Triangles correspond to the reduced-mass case (see text). [From Guillot \& Gladman 2000].

\subsubsection{Possible formation scenarii}

Three possible scenarii may explain Jupiter and Saturn's core mass and total mass of heavy elements:

1. Rapid formation with a small core or no core (Gas instability scenario). The formation of giant planets by gas instability is very fast, i.e. $10^{4}$ to $10^{5}$ years at the most (e.g. Boss 2000). Because the final mass of the planet is rapidly reached, the hydrostatic equilibrium imposes a fast contraction of the planet. Although this would have to be quantified, it appears that this planet would have a very low accretion efficiency. In order to explain the structure of Jupiter and Saturn, one needs to invoke a very large mass of solids. This scenario also would predict that Saturn has a smaller core than Jupiter, which isn't implied by the interior models. A possibility would be the capture of very small particles by the planet during their fast migration toward the Sun. All in all, this scenario cannot be ruled out but seems to be unlikely. 
2. Slow formation with extended-phase. In the model of Pollack et al. (1996), the growth of a giant planet by capture of a hydrogen-helium envelope onto a core cannot proceed extremely fast due to the feedback mechanism produced by the release of gravitational energy heating the envelope. A phase in which the planet has 20 to $30 \mathrm{M}_{\oplus}$ and its effective capture radius can be of the order of 2 to 3 times the present radius of Jupiter can persist for millions of years. This scenario is found to be consistent with the dynamical calculations. Most of the heavy elements are hence captured during the first Myr. An important consequence is that in order to explain the enrichment observed in the atmosphere, an efficient upward mixing is required.

3. Formation from an initially massive core. Another alternative could be to form a giant planet from an initially very massive core (this happens e.g. if the surface density of solids is higher than the values indicated in fig. 5.5). Part of the core would then have to be mixed upward. The advantage is that the phase of extended capture radius is not required anymore and that the formation can be considerably faster.

As discussed in section 4, the problem of mixing is a difficult one. It has been advocated by Stevenson (1982) that the energy available in Jupiter is barely sufficient to mix the any significant fraction of the core, owing to the strongly stabilizing compositional gradient. This would imply that the cores of the giant planets are primordial. Due to the properties of runaway growth that I will not discuss here (see e.g. Wuchterl et al. 2000), one would then expect the giant planets to have relatively similar cores.

However, an analysis of the accretion efficiency has shown us that the heavy elements observed in the atmosphere must have been mixed upward. If we report the 3 times solar value measured in Jupiter's atmosphere in the entire planet, this means that $18 \mathrm{M}_{\oplus}$ had to be transported upward. Efficient mixing mechanisms had therefore to exist to be capable of overcoming the gradient of molecular weight. Two possibilities exist: one is the adiabatic compressional heating during the rapid contraction phase. This phenomenon could heat water more than hydrogen and therefore ease the mixing process. Another possibility consist in advocating Kelvin-Helmoltz instability (e.g. Chandrasekhar 1961) during the accretion of the envelope: their is indeed no reason that the primordial core and the gaseous envelope would have the same angular momentum. The powerful shear would be progressively erased by mixing the different layers and transporting part of the heavy elements upward. This scenario would also explain why Jupiter would have a smaller core than Saturn: its envelope being more massive, it would be capable of mixing more of the central core. 


\section{Chapter 6}

\section{Evolution of giant planets \& brown dwarfs}

The problem of the formation of giant planets and brown dwarfs is still obscure. That of their evolution, which, as we will see, is only weakly dependent on the initial conditions, is relatively better understood. The accuracy of these calculations in fact mostly reflects our limited knowledge of their atmospheres as we will see in the next sections. They however allow us to predict probable characteristics of extrasolar giant planets, and appear to describe reasonably well brown dwarfs, based on their measured spectra.

\subsection{The virial theorem}

Integrating the hydrostatic equation leads to a well known relation linking the internal energy to the potential gravitational energy. It is very basic to stellar evolution, but I choose to rederive it here, following the formulation of Kippenhahn \& Weigert (1991), because it is fundamental to understand the evolution of substellar objects. For simplicity, rotation or the presence of magnetic fields is neglected. Starting from Eq. (2.8), we write

$$
\int_{0}^{M} 4 \pi r^{3} \frac{\partial P}{\partial m} d m=\int_{0}^{M} 4 \pi r^{3} \frac{G m}{4 \pi r^{4}} d m
$$

The left hand side can be integrated by part to yield:

$$
3 \int_{0}^{M} \frac{P}{\rho} d m=\int_{0}^{M} \frac{G m}{r} .
$$

The right hand of Eq. (6.2) corresponds to the gravitational energy with a minus sign:

$$
E_{\mathrm{g}} \equiv-\int_{0}^{M} \frac{G m}{r} d m
$$

and $-E_{\mathrm{g}}$ is the energy required to bring all the mass to infinity. The left hand side of Eq. (6.2) is related to the internal energy

$$
E_{\mathrm{i}} \equiv \int_{0}^{M} u d m=\int_{0}^{M} \frac{3}{\xi} \frac{P}{\rho} d m
$$


where $\xi \equiv 3 P / u \rho$ and $u$ is the internal specific energy. In the case of a perfect gas, $\xi=3(\gamma-1)$ where $\gamma=c_{P} / c_{V}$. In the case of a monoatomic perfect gas, $\xi=2$. If we furthermore assume that $\xi$ is uniformly constant throughout the star/planet considered, Eq. (6.2) takes the following form:

$$
\xi E_{\mathrm{i}}+E_{\mathrm{g}}=0,
$$

known as the virial theorem.

If we furthermore consider the total energy of the system $W=E_{\mathrm{i}}+E_{\mathrm{g}}$ ( $W<0$ for a gravitationally bound system), and assume that the luminosity is entirely due to the loss of energy (i.e. we neglect thermonuclear reactions, radioactivity...etc.),

$$
\frac{d W}{d t}+L=0
$$

and hence

$$
L=(\xi-1) \frac{d E_{\mathrm{i}}}{d t}=-\frac{\xi-1}{\xi} \frac{d E_{\mathrm{g}}}{d t} .
$$

This relation is valid in a variety of cases, including giant planets for their entire life and brown dwarfs for the parts of their life when thermonuclear reactions represent a small fraction of the total luminosity.

Let us consider a contracting brown dwarf or giant planet beginning its life mostly as a perfect $\mathrm{H}_{2}$ gas. In this case $\gamma \approx 7 / 5$, hence $\xi=16 / 5=3.2$. Two third of the energy gained by contraction is therefore radiated away, one third being used to increase the internal energy. This being proportional to the temperature, the effect is to heat the object. This represents the slightly counter-intuitive but well known effect that a star or giant planet initially increases its luminosity while heating up.

Let us now move further in the evolution, when the contraction has proceeded to a point where the electrons have become degenerate. The problem then becomes relatively complex because of the interplay between ions and electrons. It is instructive however to consider the ideal case, formerly valid only in the white dwarf regime, in which most of the pressure is provided by non-relativistic degenerate electrons. In that case, $P / \rho \approx(2 / 3) u$ and therefore $\xi \approx 2$ : Half of the gravitational potential energy is radiated away and half of it goes into internal energy. The problem is to decide how this energy is split into an electronic and an ionic part. The gravitational energy changes with some average value of the interior density as $E_{\mathrm{g}} \propto 1 / R \propto \rho^{1 / 3}$. The energy of the degenerate electrons is essentially the Fermi energy: $E_{\mathrm{i}} \approx E_{\mathrm{F}} \propto \rho^{2 / 3}$. One is therefore led to a simple relation between $E_{\mathrm{g}}$ and $E_{\mathrm{e}}$ :

$$
\dot{E}_{\mathrm{e}} \approx 2 \frac{E_{\mathrm{e}}}{E_{\mathrm{g}}} \dot{E}_{\mathrm{g}}=-\frac{E_{\mathrm{e}}}{E_{\mathrm{i}}} \dot{E}_{\mathrm{g}},
$$

where $E_{\mathrm{i}}$ is introduced via the virial theorem $\left(E_{\mathrm{g}}=-2 E_{\mathrm{i}}\right)$. In the case of white dwarfs, $E_{\text {ion }} \ll E_{\mathrm{e}}$ and therefore $E_{\mathrm{i}}=E_{\mathrm{ion}}+E_{\mathrm{e}} \approx E_{\mathrm{e}}$. This means that $\dot{E}_{\mathrm{e}} \approx-\dot{E}_{\mathrm{g}} \approx 2 L$. The energy balance $L=-\dot{E}_{\text {ion }}-\dot{E}_{\mathrm{e}}-\dot{E}_{\mathrm{g}}$ becomes

$$
L \approx-\dot{E}_{\text {ion }} \propto-\dot{T} .
$$

In this case, the gravitational energy lost is entirely absorbed by the degenerate electrons, and the observed luminosity is due to the thermal cooling of the ions.

For brown dwarfs and giant planets, the problem is more complex because the electrons are only partially degenerate, and the contribution of the ions to the pressure and internal energy cannot be neglected. However this only affects the solution through numerical factors: qualitatively, most of the gravitational energy lost is used up to increase the energy of the degenerate electron gas, while the luminosity is essentially provided by the cooling of the ions. 


\subsection{A semi-analytical model}

\subsubsection{Solution for isolated objects}

It is possible given certain assumptions to solve analytically the evolution problem. A more detailed numerical solution is of course eventually required, but the analytical solution is a tool to comprehend the physical problem. The solution that is presented here is due to Hubbard (1977).

We consider an already dense giant planet or brown dwarf without thermonuclear reactions, and assume that its metallic region provides the essential contribution to its cooling. Second, we will assume that it is adiabatic. One can then show that the internal temperature profile obeys a relation of the form

$$
T \approx C T_{1 \mathrm{bar}} \rho^{\gamma} .
$$

In the case of Jupiter, $C \approx 42.8$ when $\rho$ is expressed in $\mathrm{g} \mathrm{cm}^{-3}$, and $\gamma \approx 0.64$ is the Grüneisen parameter.

In the set of equations (2.41) governing the evolution of substellar objects, only the energy conservation equation involves time, through the $-T \partial S / \partial t$ term. This equation can be rewritten in the form

$$
\frac{\partial L}{\partial m}=-c_{V} \frac{\partial T}{\partial t}+c_{V}\left(\frac{\partial T}{\partial \rho}\right)_{S} \frac{\partial \rho}{\partial t}
$$

The term $(\partial T / \partial \rho)_{S}$ being positive, the luminosity is provided both by the contraction and cooling of the planet.

Let us first neglect insolation. Integrating Eq. (6.11), we obtain

$$
L=4 \pi R^{2} \sigma T_{\text {eff }}^{4}=-\int C_{V}\left(\frac{\partial T}{\partial t}-\gamma \frac{T}{\rho} \frac{\partial \rho}{\partial t}\right) d m .
$$

Furthermore, Eqs. (2.7) and (6.10) imply that

$$
\frac{\partial T}{\partial t}=T\left(-b \frac{\partial \ln g}{\partial t}+a \frac{\partial \ln T_{\mathrm{eff}}}{\partial t}+\gamma \frac{\partial \ln \rho}{\partial t}\right) .
$$

The gravity dependence is weak. The term proportional to $\partial \ln g / \partial t$ can hence be neglected. Reporting Eq. (6.13) into Eq. (6.10), one finds

$$
\begin{aligned}
d t & =-\alpha\left(T_{\mathrm{eff}}\right) T_{\mathrm{eff}}^{a-5} d T_{\mathrm{eff}}, \\
\alpha\left(T_{\mathrm{eff}}\right) & =\frac{a C K}{4 \pi R^{2} \sigma g^{b}} \int C_{V} \rho^{\gamma} d m .
\end{aligned}
$$

In the case of Jupiter, $C_{V} \approx 1.66 k_{\mathrm{B}} / m_{\mathrm{H}}$ yielding $\alpha\left(T_{\mathrm{eff}}=124.4 \mathrm{~K}\right) \approx 2.8 \times 10^{23} \mathrm{cgs}$.

Let us assume $\alpha$ constant (i.e. we neglect the evolution of the planet's structure during the contraction). The time necessary to cool from an effective temperature $T_{\text {eff }, 0}$ to $T_{\text {eff, } 1}$ is therefore

$$
\Delta t=\frac{\alpha}{4-a}\left(T_{\mathrm{eff}, 1}^{a-4}-T_{\mathrm{eff}, 0}^{a-4}\right) .
$$

Using $a \approx 1.24$, one can see that the time for the planet to cool from an infinite temperature to $T_{\text {eff }, 1}$ is approximately 50 times smaller than that required for cooling from $T_{\text {eff }, 1}$ to $T_{\text {eff, } 1} / 4$. The evolution problem is very weakly dependent on initial conditions.

Jupiter's cooling time from an initially infinite effective temperature to its present value $T_{\text {eff }}=$ $124.4 \mathrm{~K}$ is found, using eq. (6.16) to take about 5.4 Gyr. Saturn's cooling time is much shorter, i.e. about $2 \mathrm{Gyr}$. 


\subsubsection{Correction due to irradiation}

Let us now include the absorbed stellar luminosity. The total luminosity of the planet (or irradiated brown dwarf) has then three components: a directly reflected stellar part which does not contribute to the heating of the planet and is hence often not mentioned when studying the evolution; a part corresponding to the absorbed stellar luminosity, that I choose to note $L_{\mathbf{a}}$; the intrinsic luminosity $L_{\text {int }}$. The effective temperature now has to be redefined. The definition tying most closely the effective temperature to the temperature at the photosphere is

$$
4 \pi R^{2} \sigma T_{\mathrm{eff}}^{4} \equiv L_{\mathbf{o}}+L_{\mathrm{int}} .
$$

In the interior of the planet, the only relevant quantity is the intrinsic luminosity (and it thus convenient to forget the int suffix when considering the internal structure). The stellar flux is generally very rapidly absorbed and contributes in fact only to heating the outer boundary. The problem is therefore to derive the new boundary temperature. A simple approach is to use the same boundary condition [Eqs. $(2.6,2.7)]$ but with the new definition of $T_{\text {eff }}$. We will come back on that assumption when discussing the case of Pegasi planets (section 8).

We therefore rewrite Eq. (6.12) by taking account of the absorbed stellar luminosity:

$$
4 \pi R^{2} \sigma\left(T_{\text {eff }}^{4}-T_{\mathbf{\sigma}}^{4}\right)=-\int C_{V}\left(\frac{\partial T}{\partial t}-\gamma \frac{T}{\rho} \frac{\partial \rho}{\partial t}\right) d m,
$$

where $T_{\mathbf{\sigma}}$ is the effective temperature that the planet would have if its intrinsic luminosity would drop to zero while conserving the same atmosphere and overall structure. It is defined by $L_{\mathbf{o}}=$ $4 \pi R^{2} \sigma T_{\mathbf{0}}^{4}$.

It is easy to show that Eq. (6.14) is now replaced by

$$
d t=-\alpha\left(T_{\text {eff }}\right) \frac{T_{\text {eff }}^{a-5}}{1-\left(T_{\mathbf{o}} / T_{\text {eff }}\right)^{4}} d T_{\text {eff }}
$$

An expansion in powers of $\left(T_{\mathbf{\sigma}} / T_{\text {eff }}\right)^{4}$ leads to

$$
t=\frac{\alpha}{4-a} T_{\mathrm{eff}}^{a-4}\left[1+\frac{4-a}{8-a}\left(\frac{T_{\mathbf{0}}}{T_{\mathrm{eff}}}\right)^{4}+\frac{4-a}{12-a}\left(\frac{T_{\mathbf{0}}}{T_{\mathrm{eff}}}\right)^{8}+\ldots\right] .
$$

The value of $\left(T_{\mathbf{\sigma}} / T_{\text {eff }}\right)^{4}$ is 0.60 for Jupiter and 0.56 for Saturn. Using $a=1.243$, the term between the square brackets is therefore of the order of 1.3 for both Jupiter and Saturn. The contribution to the evolution of Solar radiation is hence far from negligible. Eq. (6.20) furthermore demonstrates that the evolution is slowed by the stellar radiation.

Numerically, these equations predict that Jupiter should take about 7 Gyr to cool from an infinite effective temperature to today's value. This value is however overestimated due to the fact that $\alpha$ was held constant, i.e. variations in the structure of the planet itself were neglected. Obviously, more sophisticated models have to be developed anyway to go beyond the approximations made in this semi-analytical model.

\subsubsection{Influence of a radiative zone}

The possible presence of a radiative zone can have deep, structural changes on the evolution of a planet. These modifications are not always easy to intuit. Of course, the most fundamental 
question is to know whether a radiative zone leads to a quicker cooling, or if on the contrary it slows the evolution of the planet. In fact, we shall see that the answer depends on the evolution of the radiative zone itself.

Let us consider two adiabatic models separated in time by an unknown interval $\Delta t_{\mathrm{ad}}$. Let us also consider two non-adiabatic models (possessing an internal radiative zone), which have the same external conditions as the adiabatic ones (i.e. same surface temperature, intrinsic luminosity...etc.), but separated by in time by an unknown amount $\Delta t_{\text {nad }}$. The energy conservation equation tells us that

$$
\begin{aligned}
\Delta t_{\mathrm{ad}} & \approx-\frac{M}{L} T_{\mathrm{ad}} \Delta S_{\mathrm{ad}} \\
\Delta t_{\mathrm{nad}} & \approx-\frac{M}{L} T_{\mathrm{nad}} \Delta S_{\mathrm{nad}}
\end{aligned}
$$

where $T_{\text {ad }}, T_{\text {nad }}, S_{\text {ad }}$ and $S_{\text {nad }}$ are characteristic values of temperature and specific entropy of adiabatic and non-adiabatic models, respectively.
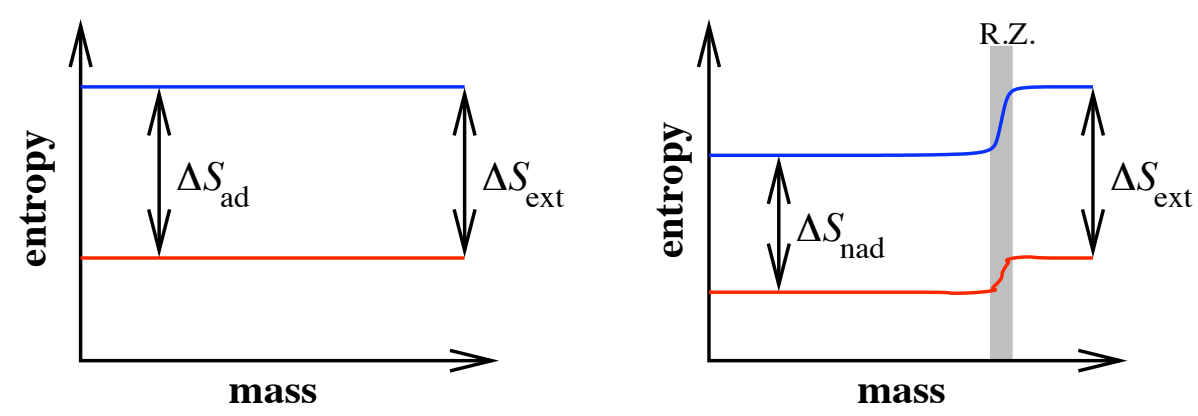

Figure 6.1: Example of evolution of the specific entropy profile in the purely adiabatic case (left) and in the presence of a radiative zone (gray area in the right hand side figure). In each case, the entropy is represented in function of the mass variable at two different times. Here the entropy jump is chosen to decrease with time. In spite of the fact that the initial surface conditions are the same in the two cases for the initial and final models, the mean entropy difference is smaller in the non-adiabatic case than in the adiabatic one $\left(\Delta S_{\text {nad }}<\Delta S_{\text {ad }}\right)$. In this example, the cooling of the non-adiabatic model is faster (see text). 
The external boundary conditions being identical, the condition of convective instability necessarily implies (neglecting small compositional differences between the two models) $T_{\mathrm{ad}}>T_{\text {nad }}$ : the radiative model is always cooler than the fully convective model. However the difference in entropy variation between the two kind of models will depend on the evolution of the characteristics of the radiative zone during the planet's cooling.

In the entirely adiabatic case, Fig. 6.1 shows that the entropy variation $\Delta S_{\text {ad }}$ between the models at ages $t_{0}$ and $t_{0}+\Delta t_{\mathrm{ad}}$ is equal to the entropy variation imposed by the external conditions $\Delta S_{\text {ext }}$. In the non-adiabatic case, the presence of a radiative zone induces a decrease of the entropy in the planet's interior. The evolution of this entropy decrease (shaded area in Fig. 6.1) is crucial. In the case of the giant planets and opacities with no alkali metals, the entropy variation in the radiative zone is greater when the planet is hotter. This implies that $\Delta S_{\text {nad }}<\Delta S_{\text {ext }}$. Consequently, for the case illustrated by Fig. 6.1, one can see that

$$
\Delta t_{\mathrm{ad}}>\Delta t_{\text {nad }}
$$

In other terms, the presence of a radiative zone tends, in this case, to accelerate the evolution.

\subsection{Evolution of Jupiter and Saturn}

\subsubsection{Results of numerical simulations}

The evolution of Jupiter and Saturn to their present state is represented on Fig. 6.2. As indicated by the analytic calculation, the initial contraction is very fast, and the initial conditions are forgotten after a few million years or less. The ages of the models which reproduce the observed radii, and effective temperature are for Jupiter 3.7 to 4.5 Gyr for models with a radiative zone, and 4.5 to $5.2 \mathrm{Gyr}$ for fully-convective homogeneous models. In the case of Saturn, these values are 2.0 to 2.4 and 2.2 to 2.6 Gyr, respectively (Guillot et al. 1995; Guillot 1999). Because, as discussed in section 4, the opacities do not account for the presence of alkali metals, one would expect values for fully-convective models to be closer to reality. These corresponds to the largest ages (and indeed, one can verify that the alkali-free opacities lead to a faster cooling, as discussed previously).

The "real" age of Jupiter and Saturn should be relatively well constrained, unless our understanding of planet formation is utterly wrong. Isotopic dating of meteorites shows that the first condensates appeared in the Solar System 4.56 Gyr ago. Jupiter and Saturn are mostly made with hydrogen and helium, and had to be formed when these elements were still present in the nebula. Observation of forming stars as well as models of circumstellar disks (see Chapter by Pat Cassen) show that hydrogen and helium should have been present for at most $\sim 10 \mathrm{Myr}$. The age of both Jupiter and Saturn must be about 4.55 Gyr.

There is therefore a problem both for Jupiter and Saturn. In the case of Jupiter, the morerealistic adiabatic models seem too old. It can be argued however that the atmospheric model is crude and introduces an uncertainty on the final age of at least $10 \%$. Imprecisions in the equations of state also introduce a probably significant uncertainty. In the case of Saturn, the discrepancy with the age of the Solar System is large and cannot be explained by inaccuracies in the calculation. Another source of energy has to be invoked: that due to the slow release of gravitational energy thus appears as the most likely one (Salpeter 1973; Stevenson \& Salpeter 1977b).

It has been suggested that deuterium-deuterium reaction in a deuterium-enhanced shell around the central core may explain "Jupiter's excess heat" (Ouyed et al. 1998). The models that I have presented explain Jupiter's luminosity naturally by the slow release of gravitational energy through the planet's contraction and cooling. The corollary is that any additional source of energy, and in 

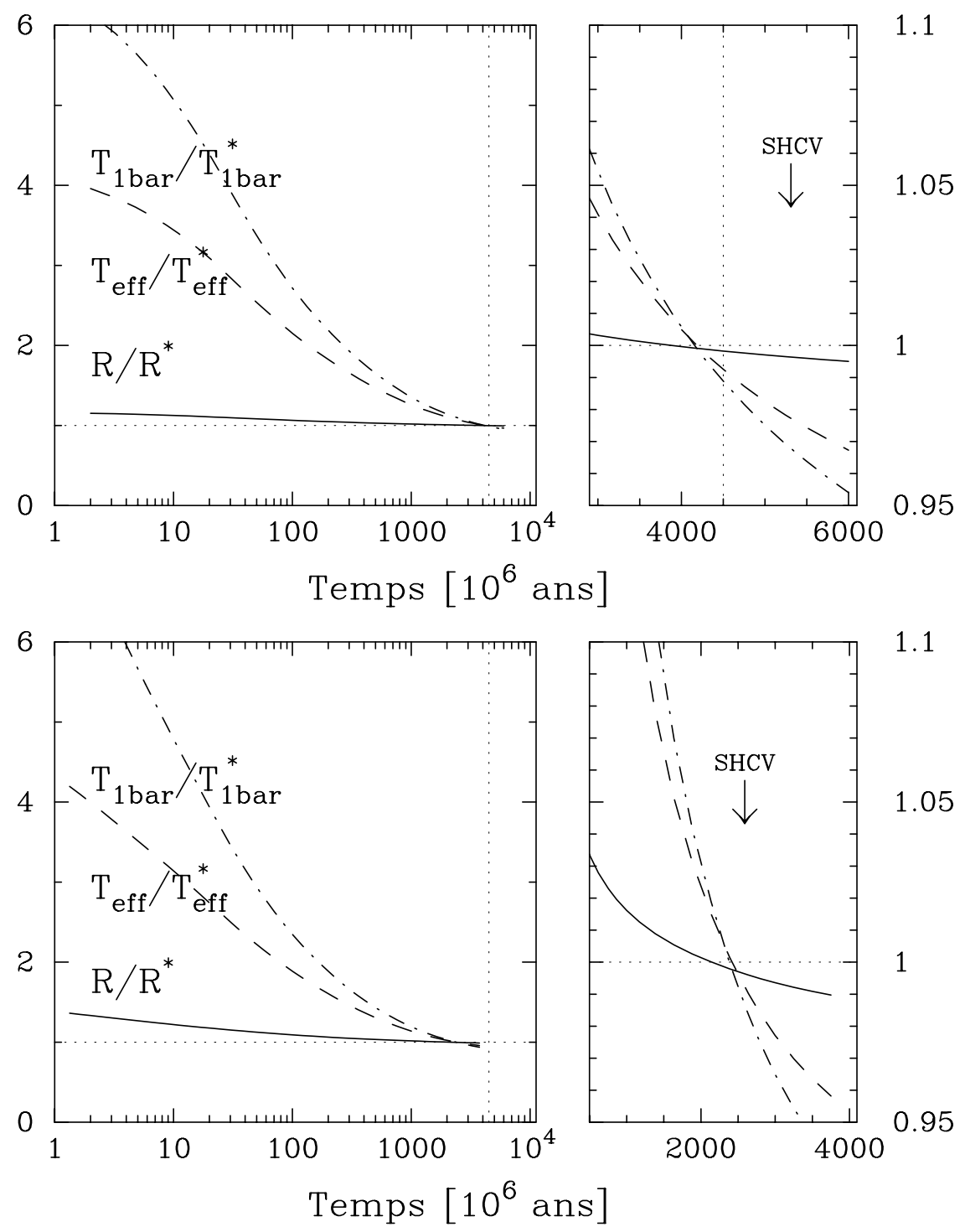

Figure 6.2: Contraction and cooling a non-adiabatic models of Jupiter (top) and Saturn (bottom) (opacities not including alkali metals). The 1 bar temperature, effective temperature and mean radius are represented as a function of time. All these quantities are normalized to their present value, $T_{1 \mathrm{bar}}^{\star}, T_{\mathrm{eff}}^{\star}$, and $R^{\star}$. The right figure is an enlargement of the left one. Note that time is then represented linearly. The vertical dotted line indicates the age of the solar system. The arrow labeled SHCV corresponds to the age obtained for a fully convective model by Saumon et al. (1992). [From Guillot et al. (1995)]. 
particular the putative D-D thermonuclear reactions would pose a very difficult problem to relate Jupiter's evolution to that of the Solar System. Furthermore, the model of Ouyed et al. requires the presence of an ad hoc deuterium shell and is therefore extremely unlikely.

\subsubsection{Including a hydrogen/helium phase separation}

The energy released by helium sedimentation per unit time can be very significant. It is of the order of

$$
L_{\text {grav }} \sim\left(\frac{d M}{d t}\right)_{\mathrm{He}} g H,
$$

where $H$ is the mean distance over which the helium has fallen. In the case of Saturn, if $10 \%$ of the helium atoms were to be transported on a distance equal to half the planet radius in $1 \mathrm{Gyr}$ then $L_{\text {grav }} \approx 4 \times 10^{24} \mathrm{erg} \mathrm{s}^{-1}$, to be compared with Saturn's present intrinsic luminosity $8.6 \times 10^{23} \mathrm{erg} \mathrm{s}^{-1}$. This process indeed provides the right amount of energy. Note that the energy is essentially proportional to the distance $H$. If the hydrogen/helium phase separation is tied to the molecular/metallic transition (see discussion in section 3), this could explain relatively naturally why this effect is more important in Saturn than in Jupiter since Saturn's metallic zone is much deeper than that of Jupiter $\left(R / R_{\text {tot }} \sim 0.43\right.$ for Saturn compared to 0.80 for Jupiter).

However, the fact that a phase separation occurs is not sufficient in itself to explain helium sedimentation. Since the planets are also convecting, the helium drops also have to grow fast enough before they are carried away by convective cells. The problem has been estimated by Stevenson \& Salpeter (1977b). First, one has to estimate the size above which the free-fall velocity of drops is larger than the convective speed. In the Stokes limit, they show that this speed is, as a function of the drop size $b$ :

$$
v_{b} \approx \frac{b^{2} g}{24 \nu}
$$

The convective velocity being of order $10 \mathrm{~cm} \mathrm{~s}^{-1}$, the free-fall time becomes larger for sizes $b \gtrsim 1 \mathrm{~cm}$.

Furthermore, the molecular diffusivity of helium in hydrogen being estimated to be $D \sim$ $10^{-3} \mathrm{~cm}^{2} \mathrm{~s}^{-1}$, the time scale for the drops to grow to $\sim 1 \mathrm{~cm}$ is found to be of order of $10^{3} \mathrm{~s}$, i.e. much faster than convective time scales.

Let us now estimate analytically how helium sedimentation affects the evolution, using an analytic model inspired by the one of section 6.2 . We assume that at a mass $m_{t}$, which can vary in time, an evolving jump of the helium mass mixing ratio $\Delta Y$ occurs. $\Delta Y$ is chosen to be positive when more helium is present at deeper levels (small values of $m$ ).

An integration the energy equation (6.12), but splitting the entropy derivative in a homogeneous and an inhomogeneous part yields

$$
L=\int-T\left[\left(\frac{\partial S}{\partial t}\right)_{Y}+\frac{d Y}{d t}\left(\frac{\partial S}{\partial Y}\right)_{t}\right] d m
$$

Neglecting the entropy of mixing and the presence of species other than hydrogen and helium implies that

$$
\left(\frac{\partial S}{\partial Y}\right)_{t}=S_{\mathrm{He}}-S_{\mathrm{H}} \equiv-\delta_{Y} S
$$

Note that the larger mass of the helium atom implies that $\delta_{Y} S$ is positive. 
Let us now assume that both $\Delta Y$ and $m_{t}$ vary with time. Mass conservation implies that

$$
\frac{d X}{d t}= \begin{cases}\Delta Y \frac{d m_{t}}{d t}+m_{t} \frac{d \Delta Y}{d t} & \text { if } m>m_{t}(t), \\ \Delta Y \frac{d m_{t}}{d t}-\left(1-m_{t}\right) \frac{d \Delta Y}{d t} & \text { if } m<m_{t}(t+d t) .\end{cases}
$$

$d X / d t$ is infinite between $m_{t}(t)$ and $m_{t}(t+d t)$ but its integral is finite:

$$
\int_{m_{t}(t)}^{m_{t}(t+d t)}-\frac{d Y}{d t} T \delta_{Y} S d m=T\left(m_{t}\right) \delta_{Y} S\left(m_{t}\right) \Delta Y \frac{d m_{t}}{d t} .
$$

We therefore obtain that the luminosity of the model at a time $t$ is the sum of three contributions:

$$
\begin{aligned}
L & =\int_{0}^{M}-T\left(\frac{\partial S}{\partial t}\right)_{Y} d m \\
& +\frac{d \Delta Y}{d t}\left\{\int_{0}^{m_{t}} T \delta_{Y} S d m-\frac{m_{t}}{M} \int_{0}^{M} T \delta_{Y} S d m\right\} \\
& -\Delta Y \frac{d m_{t}}{d t}\left\{\int_{0}^{M} T \delta_{Y} S d m-T\left(m_{t}\right) \delta_{Y} S\left(m_{t}\right)\right\}
\end{aligned}
$$

The first part is the contribution from the homogeneous evolution assumed not to change as a result of the helium sedimentation (obviously a zero-order assumption). The second part is proportional to $d \Delta Y / d t$ and is always positive if $\Delta Y$ increases with time (more helium is bought to deeper levels). The third part can be either positive or negative depending on the displacement of the transition region. The term between curved brackets is usually positive for large enough values of $m_{t}$ ( $m_{t} / M \gtrsim 0.45$ for models of Jupiter and Saturn). In this case, the contribution of this third part is thus to add to the luminosity if the transition region moves to deeper levels (small $\left.m_{t}\right)$.

Numerically one finds that

$$
\frac{1}{M}\left\{\int_{0}^{m_{t}} T \delta_{Y} S d m-\frac{m_{t}}{M} \int_{0}^{M} T \delta_{Y} S d m\right\} \approx 5 \times 10^{11} \mathrm{erg} \mathrm{g}^{-1}
$$

for both Jupiter and Saturn and

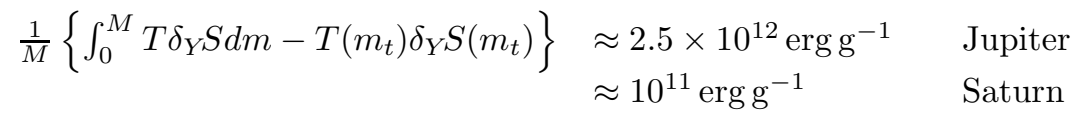

If the transition follows the PPT, then

$$
\frac{1}{M}\left|\frac{d m_{t}}{d t}\right| \lesssim 2 \times 10^{-2} \mathrm{Gyr}^{-1},
$$

and the contribution of the displacement of the transition region is negligible.

One therefore finds that the lifetime added to the planet through a phase transition from an initially homogeneous planet to one that has a helium jump $\Delta Y$ is approximately

$$
\Delta t \approx \frac{\Delta Y}{L}\left\{\int_{0}^{m_{t}} T \delta_{Y} S d m-\frac{m_{t}}{M} \int_{0}^{M} T \delta_{Y} S d m\right\},
$$


where $L$ is the planet's present intrinsic luminosity. Numerical applications indicate that

$$
\Delta t \approx 9 \Delta Y \mathrm{Gyr},
$$

for both Jupiter and Saturn. This is consistent with more detailed numerical calculations (Hubbard et al. 1999). From our evolution models, we can infer that $\Delta Y \lesssim 0.01$ for Jupiter, and that $0.2 \lesssim \Delta Y \lesssim 0.3$ in Saturn. Interior models of Jupiter are in contradiction with that upper limit since they lead to $\Delta Y \sim 0.04$ (Guillot 1999b). This problem is still not resolved. In the case of Saturn, this implies that the Voyager value for the atmospheric helium mixing ratio is too low, something recognized independently from interior models (Guillot 1999a), evolutionary models (Hubbard et al. 1999) and a reanalysis of Voyager IRIS data (Conrath \& Gautier 2000).

\subsection{From giant planets to brown dwarfs}

Giant planets and brown dwarfs share the same physics. It is so much the case that it is difficult to make the distinction between the two classes of objects. Presumably they should be formed by different mechanisms (see e.g. Wuchterl, Guillot \& Lissauer 2000). However, I will here arbitrarily define giant planets as substellar objects in which thermonuclear reactions do not occur, and brown dwarfs as objects which burn some deuterium during their life, but which never attain the equilibrium phase (main sequence) in which most of their energy is provided by hydrogen burning.

\subsubsection{Nuclear reactions}

For brown dwarfs, the occurrence of thermonuclear reactions is almost entirely due to a truncated PPI cycle (e.g. Burrows \& Liebert 1993):

$$
\begin{aligned}
& \mathrm{p}+\mathrm{p} \rightarrow d+e^{+}+\nu_{e} \\
& \mathrm{p}+\mathrm{d} \rightarrow{ }^{3} H e+\gamma
\end{aligned}
$$

Note that because of the relatively low central temperatures, only ${ }^{3} \mathrm{He}$, not ${ }^{4} \mathrm{He}$ is formed through these reactions. The $p p$ and $p d$ reactions release 1.442 and $5.494 \mathrm{MeV}$, respectively.

The energy released through these reactions is, assuming no screening (Fowler et al. 1975):

$$
\dot{\epsilon}_{p p}=2.5 \times 10^{6} \frac{\rho X^{2}}{T_{6}^{2 / 3}} e^{-33.8 / T_{6}^{1 / 3}} \operatorname{erg~g}^{-1} \mathrm{~s}^{-1}
$$

and

$$
\dot{\epsilon}_{p d}=1.4 \times 10^{24} \frac{\rho X Y_{d}}{T_{6}^{2 / 3}} e^{-37.2 / T_{6}^{1 / 3}} \operatorname{erg~g}^{-1} \mathrm{~s}^{-1},
$$

where $X$ and $Y_{d}$ are the mass mixing ratios of hydrogen and deuterium, respectively, and $T_{6}=$ $T / 10^{6} \mathrm{~K}$. Note that the primordial deuterium abundance is of the order of $Y_{d}=2 \times 10^{-5}$.

However, Eqs. $(6.36,6.37)$ are underestimates because the fact that the plasma is strongly coupled significantly softens the repulsive potential of the nuclei. A detailed analysis of this is discussed by Saumon et al. 1996 and Chabrier \& Baraffe (1997). In the case of brown dwarfs, the enhancement factor is of the order of $\sim 2$ for both reactions. An estimate of the final sensitivity of the reactions to temperature and density variations is provided by Burrows \& Liebert (1993):

$$
\dot{\epsilon}_{n} \approx 5.9 \times 10^{10}\left(\frac{T}{3 \times 10^{6} \mathrm{~K}}\right)^{6.3}\left(\frac{\rho}{10^{3} \mathrm{~g} \mathrm{~cm}^{-3}}\right)^{1.28} \mathrm{erg} \mathrm{g}^{-1} \mathrm{~s}^{-1} .
$$


This expansion around $T=3 \times 10^{6} \mathrm{~K}$ and $\rho=10^{3} \mathrm{~g} \mathrm{~cm}^{-3}$ shows the strong sensitivity of the energy production to the temperature. Note however that other thermonuclear reactions can have much steeper temperature dependences in the case of more massive stars (e.g. Clayton 1963).

\subsubsection{Brown dwarf models and results}

The two most cited evolution models of giant planets and brown dwarfs to date are those of the "Tucson group" (e.g. Burrows et al. 1997) and of the "Lyon group" (e.g. Chabrier \& Baraffe 1997). These models share many similarities, and in particular have the same equation of state and same nuclear burning rates. They however differ on a few points:

- On the atmospheric model: The 'Tucson' model uses the k-coefficient approach from Marley et al. (1996) and an approximate treatment of clouds using Lunine et al. (1989). The 'Lyon' model is based on a detailed line by line approach (e.g. Allard \& Hauschild 1995).

- On the treatment of convection: The 'Tucson' model is essentially adiabatic, whereas the 'Lyon' one uses the mixing length theory.

- On conduction: implemented in the 'Lyon' model only, using conductive opacities from Potekhin et al. (1999). Figure 6.3 shows the evolution of the conductive core of a $0.06 \mathrm{M}_{\odot}$ brown dwarf (from Chabrier et al. 2000b).

Other models by D'Antona \& Mazitelli (see Montalbán et al. 2000) are available but will not be discussed since they have generally concerned objects of larger masses.

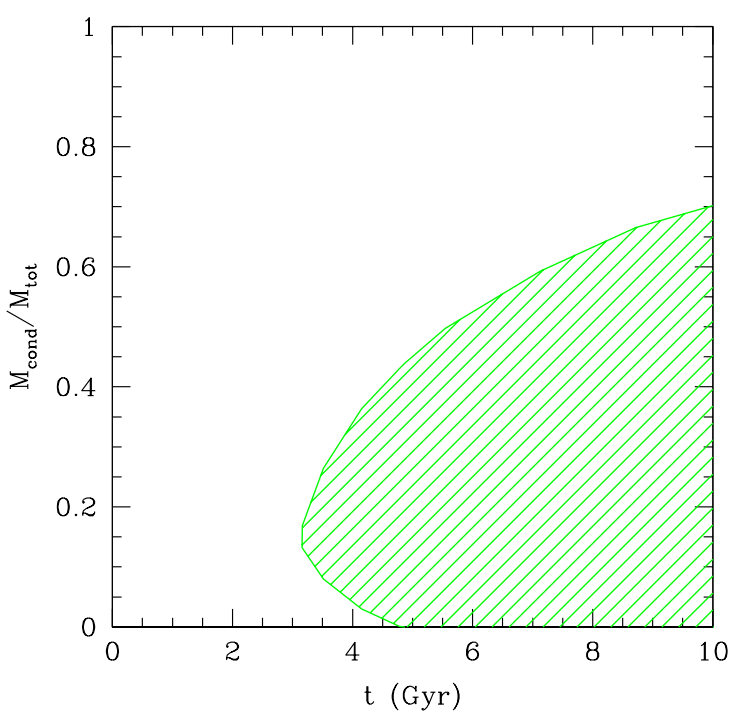

Figure 6.3: Evolution of the conductive core Mcond/Mtot (shaded area) as a function of time for a $0.06 \mathrm{M}_{\odot}$ brown dwarf. [From Chabrier et al. (2000b)]. 
Figures 6.4 and 6.5 show the evolution of isolated giant planets, brown dwarfs and stars. The distinction between stars and brown dwarfs can be readily seen from the fact that stars reach a long equilibrium period during which the tendency of the star to contract under the action of gravity is balanced by thermonuclear hydrogen fusion. For brown dwarfs, even if thermonuclear reactions are indeed possible (even of hydrogen for the most massive ones), they are never energetic enough to reach this balance: brown dwarfs and giant planets contract inexorably. Note that the crossing of evolution lines in fig. 6.4 is due to deuterium burning. It occurs later for brown dwarfs of small masses $\left(\sim 15 \mathrm{M}_{\mathrm{J}}\right)$, and those can hence be, for a small period of time, more luminous than slightly more massive brown dwarfs that have already consumed all their deuterium.

Figure 6.5 shows the relative constancy of the radius both as a function of time and mass, as well as the range of effective temperatures spanned by brown dwarfs and isolated giant planets. After 0.1 Gyr of evolution, it is found that all isolated brown dwarfs and giant planets should have a radius ranging between $10^{10}$ and $5 \times 10^{9} \mathrm{~cm}$. For comparison, Jupiter's mean radius is $7 \times 10^{9} \mathrm{~cm}$. The effective temperatures can range from about $3000 \mathrm{~K}$ for a young $(\sim 10 \mathrm{Myr})$ massive brown dwarf to only $\sim 100 \mathrm{~K}$ for a 5 Gyr isolated Jupiter-mass planet.

Figure 6.6 is a theorist's H-R diagram for the "brown dwarfs" and giant "planets." The inset is a continuation of the figure down to low luminosities and $\mathrm{T}_{\text {eff }} \mathrm{s}$. The current Jupiter and Saturn are superposed for comparison (Pearl \& Conrath 1991). Importantly, constant mass trajectories never cross and it is only for objects below $25 \mathrm{M}_{\mathrm{J}}$ that temperatures below $400 \mathrm{~K}$ are reached within $10^{10}$ years. All substellar objects decrease in luminosity monotonically, though during the early phases deuterium burning slows the evolution. As the "brown dwarfs" and "planets" cool to their cold radii, their tracks in the lower right of the H-R diagram correspond closely to curves of constant radius.

The consequences of a different helium to hydrogen ratio, of rotation or of the presence of a central dense core on the final luminosity and radius of 1 and $5 \mathrm{M}_{\mathrm{J}}$ planets are indicated by Saumon et al. (1996). They show that a 10\% variation of $Y$ generally translate into a $5 \%$ variation of $R$ and $L$ ( $Y$ and $R$ being always anti-correlated, while $Y$ and $L$ are generally correlated). The presence of rapid rotation (with rotational speeds similar to that of Jupiter) can also significantly affect both the radius, increasing it by up to $20 \%$, and the luminosity, which can be decreased by the same amount (but complex behavior can be found). 


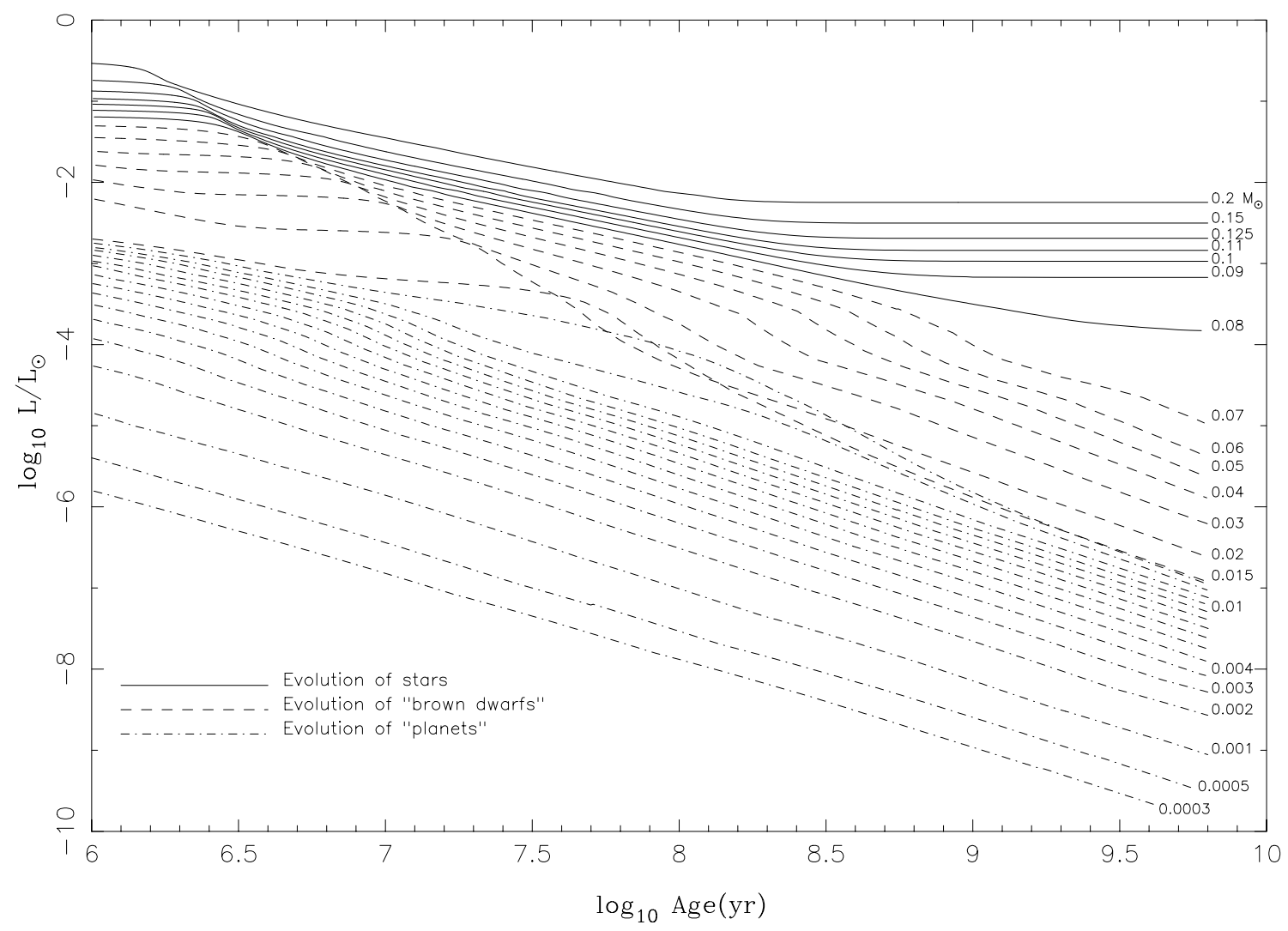

Figure 6.4: Evolution of the luminosity (in $\mathrm{L}_{\odot}$ ) of solar-metallicity $\mathrm{M}$ dwarfs and substellar objects vs. time (in yr) after formation. The stars, "brown dwarfs" and "planets" are shown as solid, dashed, and dot-dashed curves, respectively. In this figure, we arbitrarily designate as "brown dwarfs" those objects that burn deuterium, while we designate those that do not as "planets." The masses (in $\mathrm{M}_{\odot}$ ) label most of the curves, with the lowest three corresponding to the mass of Saturn, half the mass of Jupiter, and the mass of Jupiter. [From Burrows et al. (1997)]. 


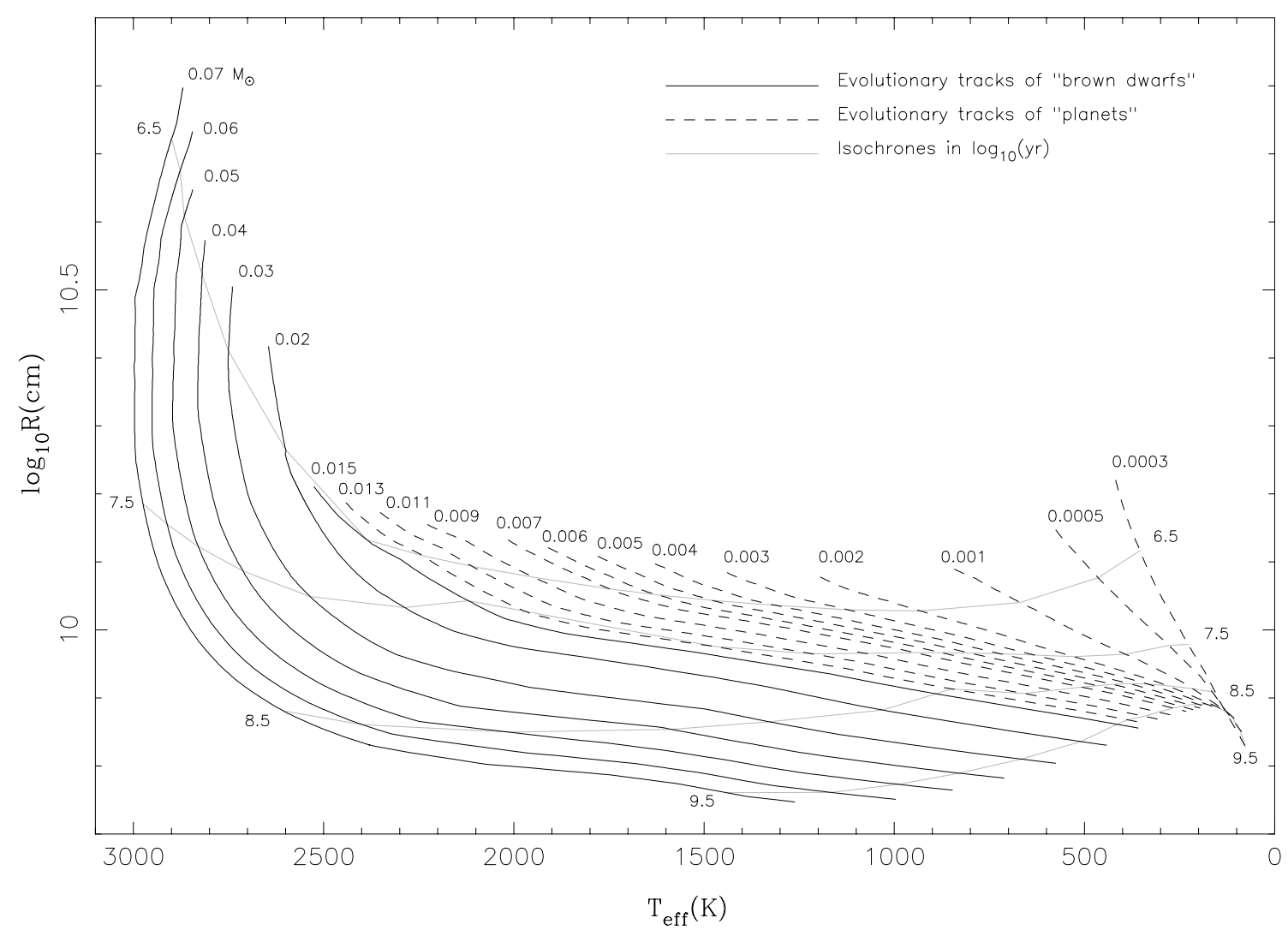

Figure 6.5: $\log 10$ radius (in $\mathrm{cm}$ ) vs. effective temperature (Teff, in K), with Teff decreasing to the right. The isochrones are the almost horizontal lines and are labeled in $\log 10 \mathrm{yr}$. In all cases, the radius decreases with time. Initially, for the more massive brown dwarfs, the effective temperature is roughly constant, or slightly increasing, before decreasing inexorably at later times. [From Burrows et al. (1997)]. 


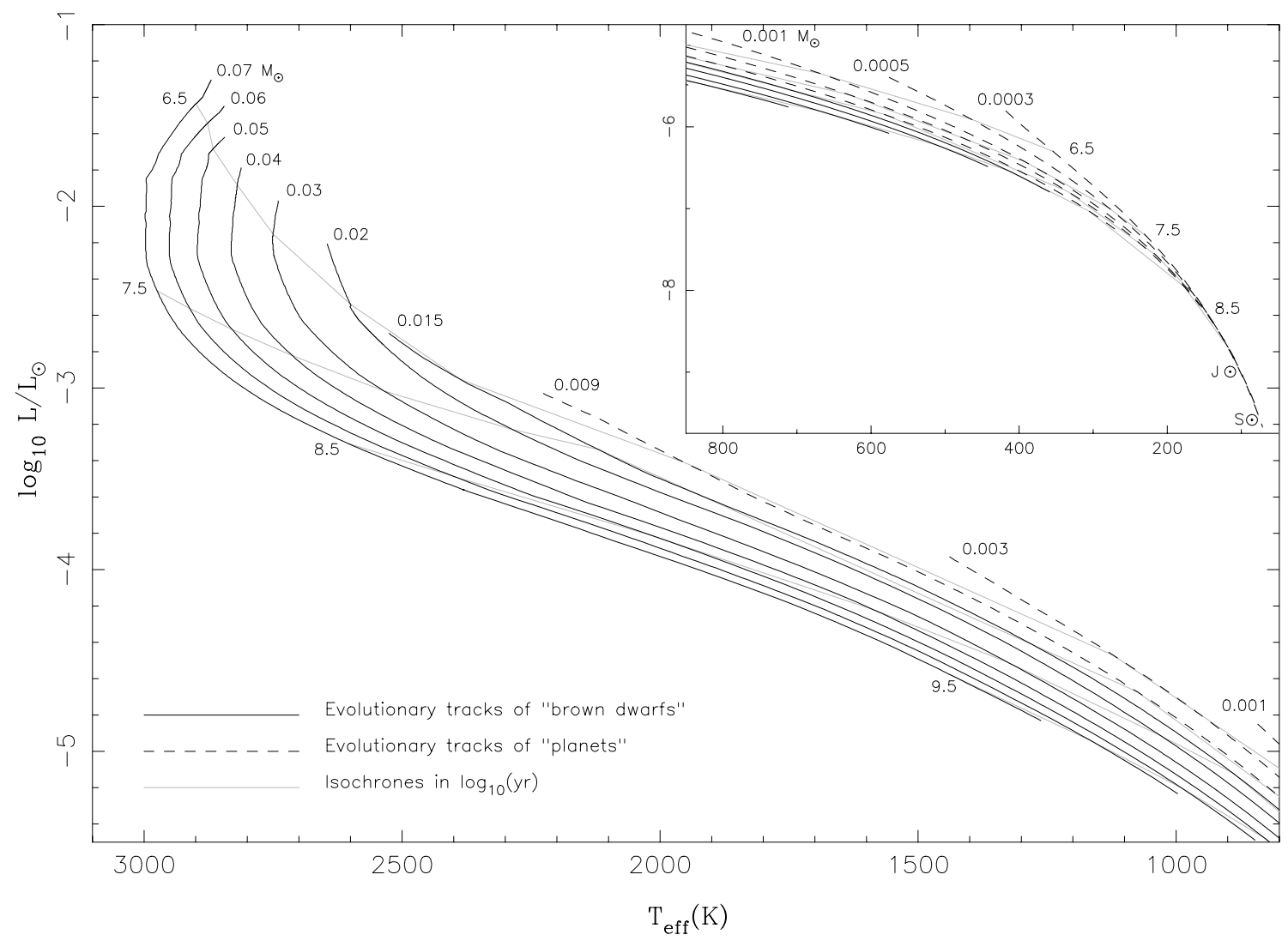

Figure 6.6: $\mathrm{H}-\mathrm{R}$ diagram: luminosity (in $L_{\odot}$ ) versus $\mathrm{T}_{\text {eff }}$ (in $\mathrm{K}$ ) for various masses labeled on the figure in $\mathrm{M}_{\odot}$. Due to the large range in luminosity and the near degeneracy of the tracks of substellar objects at late stages of evolution, it is not possible to represent with adequate detail the whole H-R diagram as one figure. Accordingly, the low-temperature and low-luminosity tail of the H-R diagram is shown in the inset. The observed positions of Jupiter and Saturn are labeled as points "J" and "S," respectively. [From Burrows et al. (1997)]. 


\subsubsection{Deuterium, lithium and hydrogen burning}

The calculation of central temperatures shows at what masses and when various key-species are burned in brown dwarfs and stars. Because thermonuclear reactions are mostly temperature dependent, fig. 6.7 shows as horizontal lines the burning temperatures of deuterium, lithium and hydrogen. It can be seen that objects of $0.012 \mathrm{M}_{\odot}\left(\sim 12 \mathrm{M}_{\mathrm{J}}\right)$ fail to reach the deuterium-burning limit. Using this property to distinguish brown dwarfs and planets sets the realm of brown dwarfs beyond $13 \mathrm{M}_{\mathrm{J}}$ (see Burrows et al. 1997; Chabrier et al. 2000a).

In objects of about $0.06 \mathrm{M}_{\odot}$, lithium starts burning. The signature of lithium in the spectrum of an object is thus an important sign to prove its substellar nature. There are various caveats however: at low temperatures, lithium pairs with hydrogen to form the mostly undetectable $\mathrm{LiH}$ (Lodders 1999). Furthermore, stars retain some of their primordial lithium for a few million years for the less massive of them. Therefore, lithium can be observed in young objects without these being brown dwarfs.

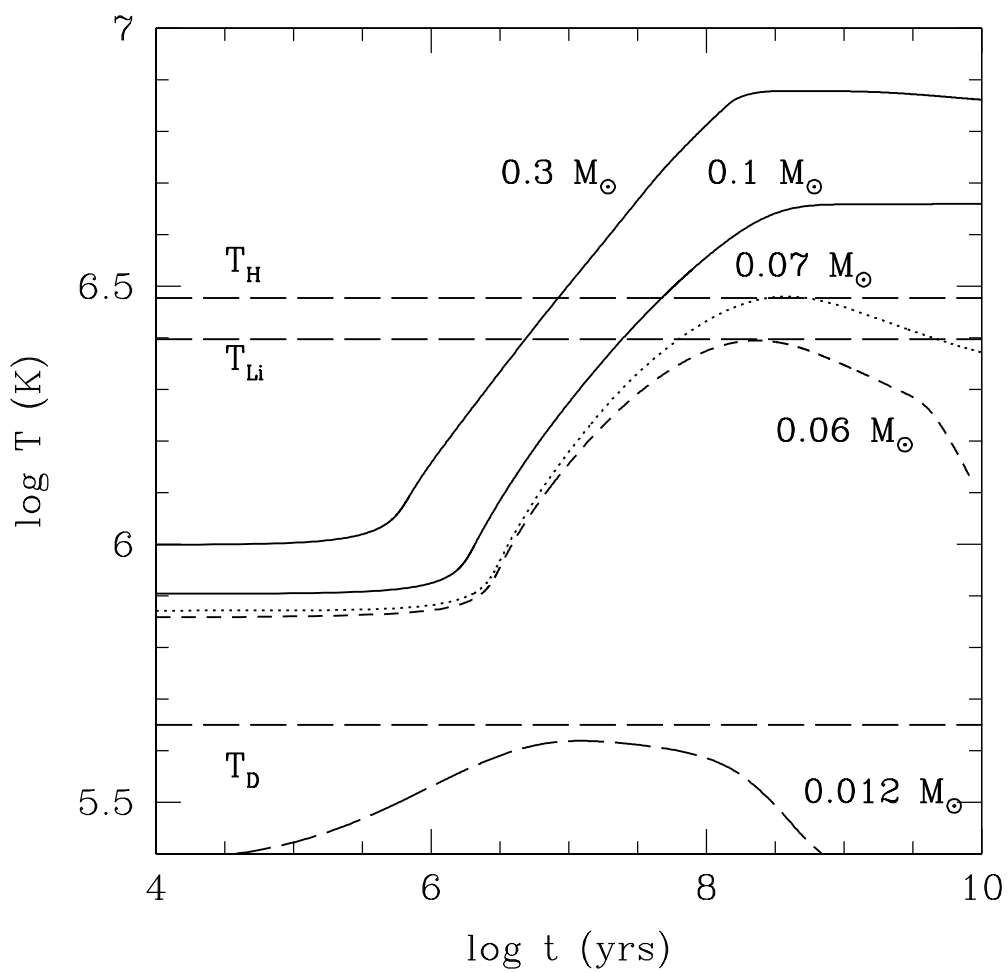

Figure 6.7: Central temperature as a function of age for different masses. $T_{\mathrm{H}}, T_{\mathrm{Li}}$ and $T_{\mathrm{D}}$ indicate the hydrogen, lithium and deuterium burning temperatures, respectively. [From Chabrier \& Baraffe 2000]. 
Hydrogen starts burning for masses higher than $0.7 \mathrm{M}_{\odot}$. This however depends on the metallicity of the object: this limit is valid for solar-metallicity, but goes up to $0.8 \mathrm{M}_{\odot}$ for objects with $[\mathrm{Fe} / \mathrm{H}]=-2$ (Chabrier \& Baraffe 2000). This can be understood as follows. We have seen that the photospheric pressure is proportional to $g / \kappa$. Objects with a smaller metallicity, hence smaller atmospheric $\kappa$ have a larger photospheric pressure, for a given gravity. They hence have a colder interior at a given pressure. One therefore needs to go to higher gravities, hence higher masses to reach a central temperature above the deuterium fusion point.

\subsection{Extrasolar giant planets}

The extrasolar planets that have been discovered so far by the radial velocimetry technique orbit all relatively close to a star; the amount of radiation that they receive has to be taken into account. I present here results obtained in the weak-irradiation approximation discussed in section 6.2.2: these results are valid when most of the stellar flux is absorbed in a convective, adiabatic zone. We will see however that this is not true of strongly irradiated planets: in this case specific calculations have to be performed (section 8 )

Figure 6.8 gives examples of effective temperatures and radii predicted for some of the recently found extrasolar giant planets and brown dwarfs, assuming solar composition, a factor 2 uncertainty on the mass (due to the fact that radial velocity measurements only yield $M \sin i$, where $i$ is the inclination of the orbital plane), and including uncertainties on the ages and albedo (between 0.1 and 0.5). It illustrates the diversity of planets detected so far. Because of the range of temperatures, many different condensates (from ammonia to silicates) are expected in planetary atmospheres. However, the calculated radii are always close to that of Jupiter, until the mass is large enough to sustain hydrogen fusion, at about $75 \mathrm{M}_{\mathrm{J}}$. A local maximum of the radius at a mass of $\sim 4 M_{J}$ for isolated planets is due to the competition between additional volume and increased gravity. (This is because, when considering planets of larger masses, the degenerate metallic hydrogen region grows at the expense of the molecular region.) Planets that are significantly heated by their star have larger radii for smaller masses because their cooling is strongly suppressed. This case will be discussed in more detailed in section 8, in connection with the constraints obtained for HD209458b. 

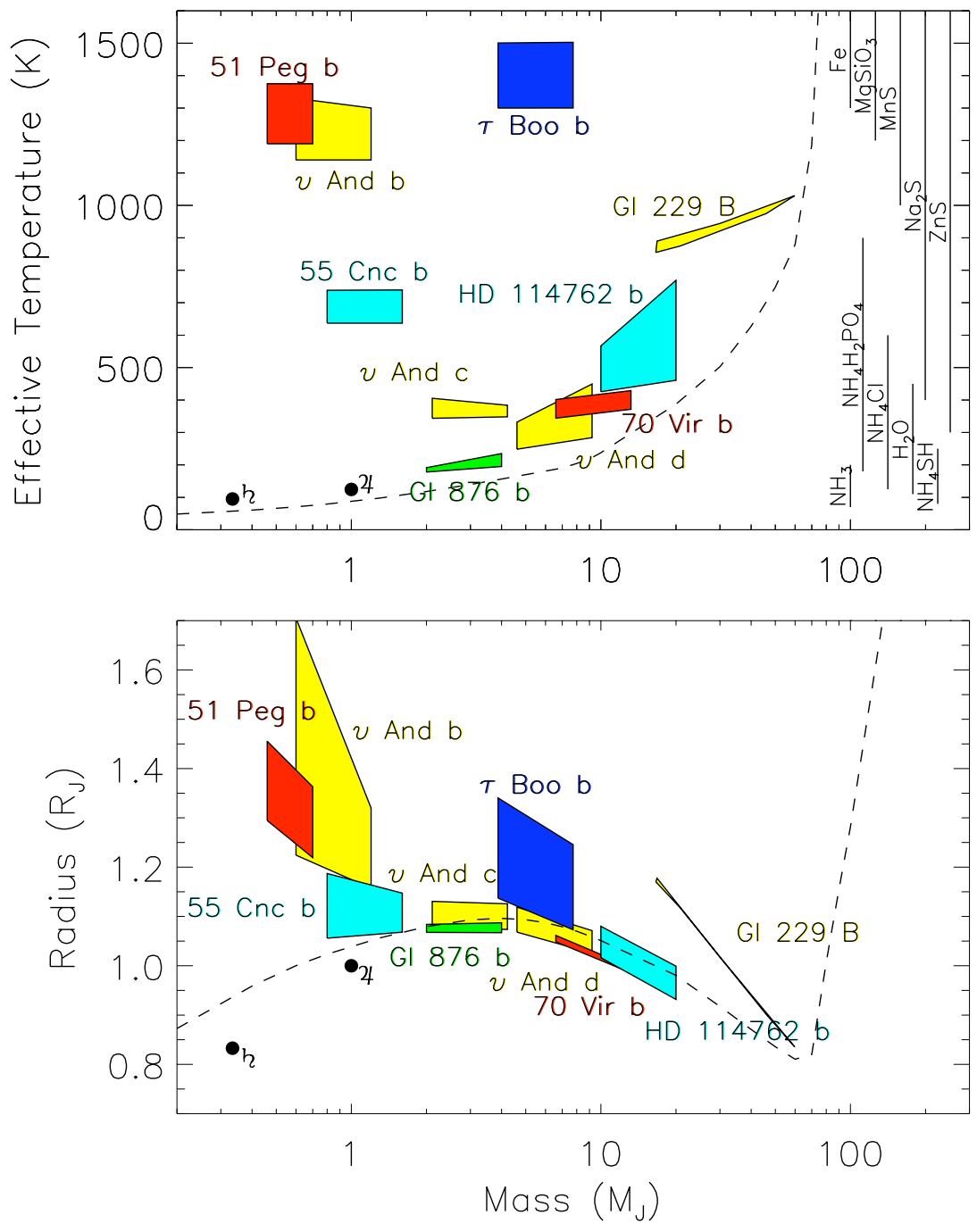

Figure 6.8: Predicted effective temperatures and radii (in units of Jupiter radii, $R_{\mathrm{J}} \sim 70,000 \mathrm{~km}$ ) of some extrasolar planets and brown dwarfs, including reasonable uncertainties for their mass, albedo and age (see text). The dashed line is for isolated $\mathrm{H}-\mathrm{He}(Y=0.25)$ objects after $10 \mathrm{Ga}$ of evolution. The upper panel also shows potentially important chemical species expected to condense near the photosphere in the indicated range of effective temperatures. [From Guillot (1999b)] 


\section{Chapter 7}

\section{Spectra \& Atmospheres}

\subsection{Direct Observations of Substellar Objects}

\subsubsection{Gliese 229 B}

Numerous observations of brown dwarfs are now available, but the first object whose substellar nature has been recognized as such beyond any doubt is Gliese $229 \mathrm{~B}$. That object was discovered in 1995 (Nakajima et al. 1995; Oppenheimer et al. 1995), but it is still one of the most studied object of the field. It has the particularity of being companion to a cool M1-dwarf star, only 5.77 parsecs away. Its projected separation is only $45 \mathrm{AU}$, i.e. about the distance between Pluto and the Sun. (Note that its real mean orbital distance is still unknown.) One of the key features of the spectrum of Gl229B was the presence of methane absorption. Because this molecule turns into $\mathrm{CO}$ at temperatures above 1000 to $1500 \mathrm{~K}$ for realistic photospheric pressures (see section 7.2.3 hereafter), this implied that Gl229B was a genuine brown dwarf.

Two theoretical attempts to model the brown dwarf's spectrum were performed independently by Allard et al. (1996) and Marley et al. (1996). These works concluded to an effective temperature $T_{\text {eff }} \sim 900 \pm 100 \mathrm{~K}$ and a gravity $\log g \sim 5 \pm 0.5$ with $g$ in $\mathrm{cm} \mathrm{s}^{-2}$. They identified several water and methane bands, and were able to correctly reproduce most of the spectrum. Another work from Tsuji et al. (1996), also coincided with the discovery. The comparison of this work with the observations showed that clouds were not present, in Gl229B, or that they were patchy. A few problems were however found at the time:

- The fit to the observed spectrum were relatively poor in the region of methane absorption. This problem is still present, in the lack of reliable opacity data for that molecule at temperatures of $\sim 1000 \mathrm{~K}$ and more.

- The predicted fluxes were much too large, by several orders of magnitude, for wavelength $\lambda \lesssim 1 \mu \mathrm{m}$. The works of Tsuji et al. and Allard et al. did not include the condensation and settling of the very efficient absorber TiO: their fluxes were coincidentally similar to the observed ones, but for the wrong reasons. None of the distinctive features of $\mathrm{TiO}$ absorption have indeed been found in Gl229B's spectrum (Oppenheimer et al. 1998). Marley et al. used an arbitrary cutoff of the absorption for $\lambda<1 \mu \mathrm{m}$. It was later advocated that photochemical processes due to the irradiation of the weak Gl229A could create large-enough particles to explain that absorption but no component could be found to provide the right slope (Griffith 

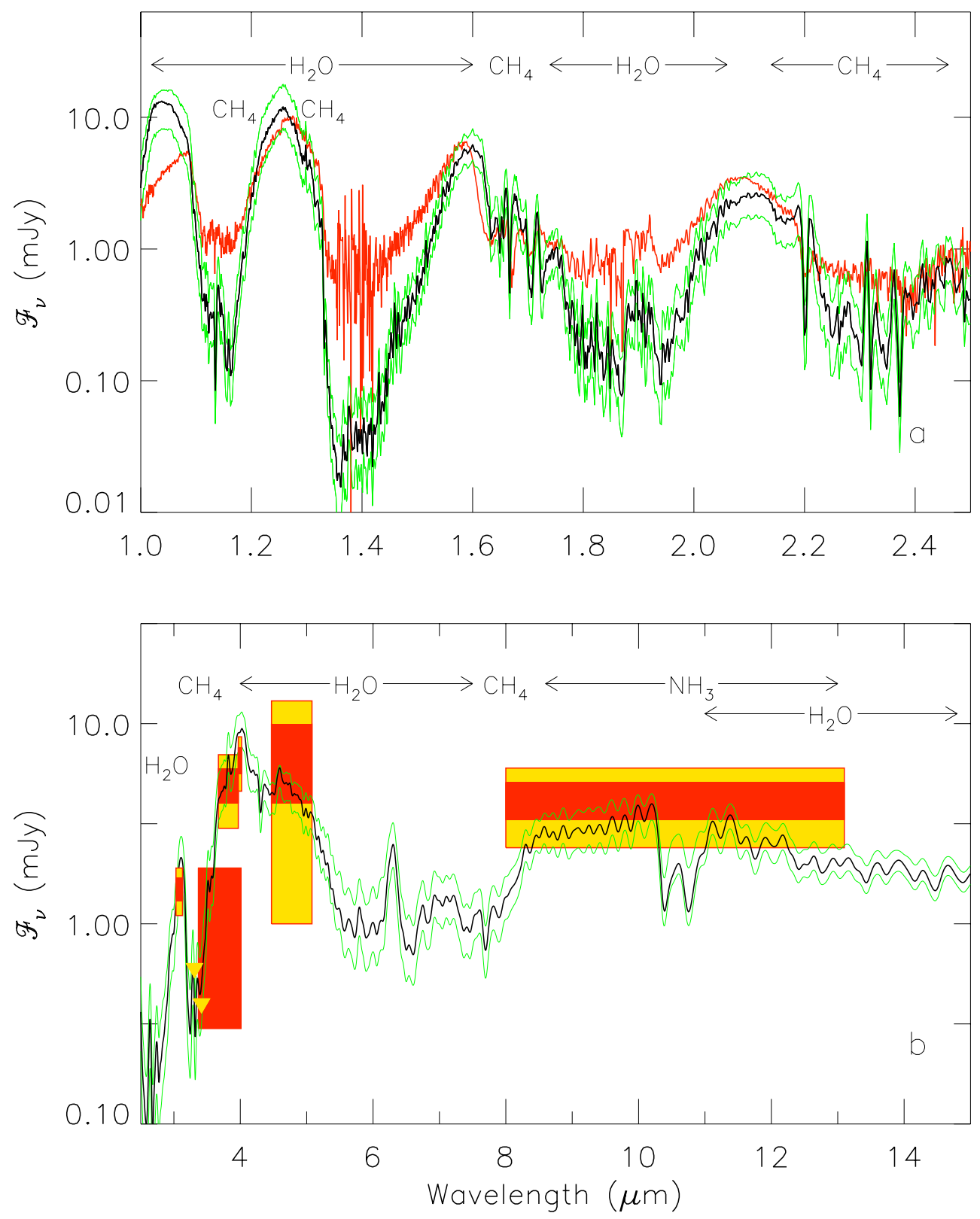

Figure 7.1: Synthetic spectra for (bottom to top) $T_{\text {eff }}=890 \mathrm{~K}, 960 \mathrm{~K}, 1030 \mathrm{~K}$ and $g=10^{5} \mathrm{~cm} \mathrm{~s}^{-2}$ together with observed data from Geballe et al. (1996) (top panel) and photometric broadband measurements also from Geballe et al. (bottom panel). In both panels, spectral intervals are labeled with the molecules primarily responsible for the opacity in that interval. [From Marley et al. 1996]. 
et al. 1998). The problem was later shown quite convincingly to be due to the absorption of alkali metals, and in particular to the potassium doublet (Burrows et al. 2000a). Although this theory depends on an ad hoc parameter, the slope of the non-lorentzian line profile (see section 4.2.3), it has been shown to properly reproduce the spectrum of another cool brown dwarf (Liebert et al. 2000).

- The theoretical spectra predict absorption minima that are much deeper (by one to two orders of magnitude) than the observed one. Although one possible explanation could be the contamination of the observations by light scattered from Gl229A, a careful analysis of the measured spectrum (e.g. Oppenheimer et al. 1998) shows that this is unlikely. No convincing explanation has yet been proposed to explain the discrepancy.

\subsubsection{The new spectral classes}

As discussed in the introduction, the technological progresses made possible the detection of hundreds of brown dwarfs in only a few years. This led to the definition of a new spectral type, the first one in more than a hundred years. This spectral type, "L" was first proposed by Martín et al. (1997), and later worked out by Kirkpatrick et al. (1999) and Martín et al. (1999). I will not discuss in detail the classification itself (or rather the classifications, there being some divergences between the two groups). In a nutshell, M-type stars are identified by distinctive signatures of molecules, especially $\mathrm{TiO}$ and VO. The spectra of L-type objects see the progressive disappearing of $\mathrm{TiO}$ and $\mathrm{VO}$ lines and the advent of $\mathrm{K}, \mathrm{H}_{2} \mathrm{O}$, Cs...etc. Cooler than the L-dwarfs, one then finds the T-dwarfs, whose detailed classification scheme has obviously to be worked out, but which are characterized by the presence of $\mathrm{CH}_{4}$ absorption. Finally, it has been proposed that even cooler objects similar to Jupiter and Saturn (who mainly show features of $\mathrm{CH}_{4}$ and $\mathrm{NH}_{3}$ ) be termed "Y-dwarfs" (see Basri 2000 for a review).

The correspondence between hydrogen-burning and spectral-type is not a simple one because it depends on factors such as the gravity and metallicity of the object. For solar composition, the limit between stars and brown dwarfs is at M10 around $1 \mathrm{Gyr}, \mathrm{M} 7$ around $100 \mathrm{Myr}$, and M6 around $10 \mathrm{Myr}$.

\subsubsection{The colors of brown dwarfs}

The abundance of brown dwarfs now discovered makes us almost forget how faint these objects are, and how difficult it is to find them. They are indeed 10,000 to 100,000 times fainter than our Sun. Since high-resolution spectra imply long exposures, programs aiming at the detection of brown dwarfs have relied on color information, i.e. images taken with several broadband filters that combined together provide information on the effective temperature of the targets. Because of the coolness of the sources, brown-dwarf surveys (e.g. DENIS, 2MASS) have generally been done using infrared I, J and K bands (see Basri 2000).

It is a well known property of blackbodies that they get redder and redder as one looks at objects that are cooler and cooler: the peak of the Planck function is then displaced to longer wavelengths. This implies that, e.g. relative to the $\mathrm{K}$ band, less flux is emitted in the $\mathrm{J}$ band. Because astronomers are nostalgic of outdated conventions, this implies an increasing $\mathrm{J}-\mathrm{K}$ value $(\mathrm{J}$ and $\mathrm{K}$ being the magnitudes in the $\mathrm{J}$ and $\mathrm{K}$ bands, respectively).

Figure 7.2 shows a color-magnitude diagram in which theoretical calculations are compared to observations. Main sequence stars are easily spotted by their low, relatively uniform J-K value. This is due to the fact that at high temperatures, the $\mathrm{J}$ and $\mathrm{K}$ bands are in the Wien tail of the 
Planck function and their difference is independent of temperature. Objects of smaller absolute magnitude $M_{\mathrm{K}}$ then progressively move to the red-part (right) of the diagram. This tendency is well reproduced by a "dusty" atmospheric model, i.e. one in which all the condensed particles are assumed to remain in the atmosphere. The effect of the presence of dust is effectively to reduce spectral variations so that the spectrum is more similar to that of a black body (Allard et al. 2001).

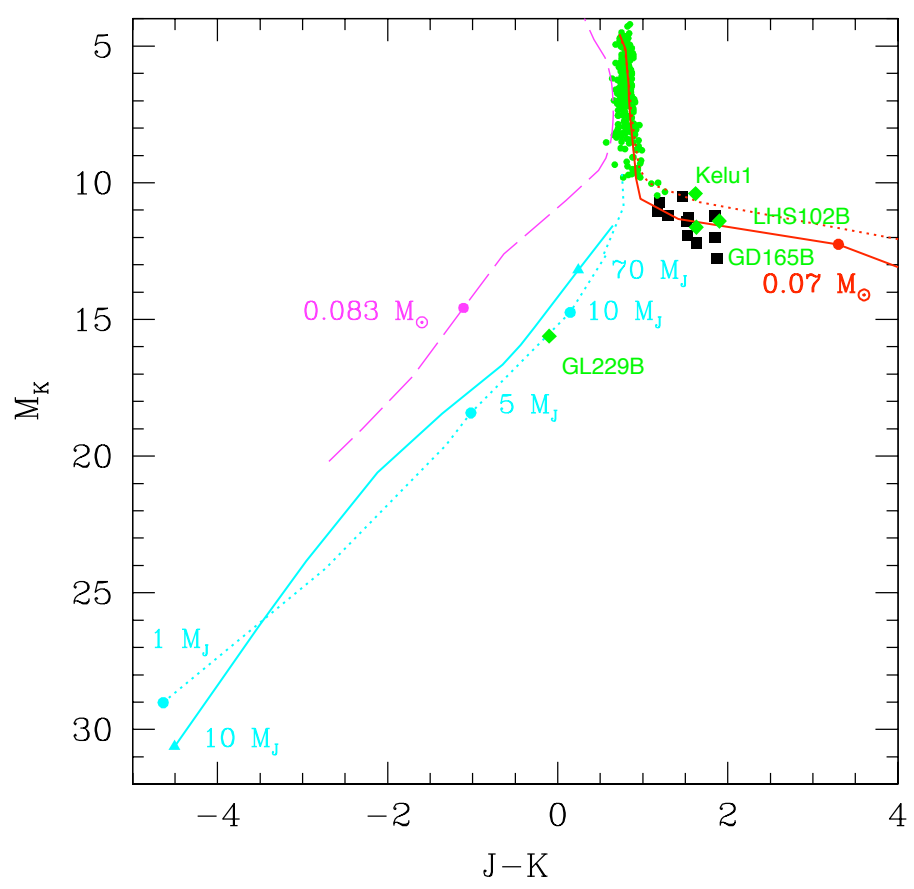

Figure 7.2: $\mathrm{M}_{K}$ vs $(J-K)$ diagram for different ages and metallicities: $[\mathrm{Fe} / \mathrm{H}]=0$ for $10^{8} \mathrm{yr}$ (dotted lines) and $5 \times 10^{9} \mathrm{yr}$ (solid lines); $[\mathrm{Fe} / \mathrm{H}]=-2, \mathrm{t}=10 \mathrm{Gyr}$ (dashed line). The red curves on the right correspond to the DUSTY models for $[\mathrm{Fe} / \mathrm{H}]=0$. The blue curves on the left correspond to the COND models for $[\mathrm{Fe} / \mathrm{H}]=0$. Filled circles and triangles on the isochrones indicate masses either in $\mathrm{M}_{\odot}$ or $M_{\mathrm{J}}\left(1 M_{\mathrm{J}} \approx 10^{-3} \mathrm{M}_{\odot}\right)$.[From Chabrier \& Baraffe 2000].

However, at still lower temperatures, Gl229B sticks clearly out of this tendency, and is in fact significantly bluer in J and $\mathrm{K}$ than main sequence stars! Furthermore, it is not an isolated case: several other cool brown dwarfs have now been detected to have the same characteristics (e.g. Burgasser et al. 1999; Strauss et al. 1999). As we will see, this rapid variation in color is indicative of a transition from "dusty" to "clear" atmospheres, probably sharpened by the additional cooling provided by the apparition of methane at low temperatures.

\subsubsection{Detection of very young substellar objects}

Brown dwarfs can be discovered in the field, as for the DENIS and 2MASS surveys. They can also be discovered in known star-forming regions: because they are young, they can be considerably 
hotter than the average field objects. However, most of these regions are relatively far away (100's of parsecs), and extinction then becomes a problem.

A very interesting region is the $\sigma$-Orionis cluster, which is only a few Myr old. Very faint, low-mass objects have been successfully discovered (see Martín et al. 2001 and references therein). These authors have shown that a continuous sequence of brown dwarfs is present, down to very low masses (perhaps $\sim 8 \mathrm{M}_{\mathrm{J}}$ ). Similar results have been obtained for other clusters, were numerous brown dwarfs were detected down to and beyond the deuterium burning limit. Mass functions down to these low masses have been derived for the IC348 cluster (Najita, Tiede, \& Carr 2000) and the Trapezium (Hillenbrand \& Carpenter 2000; Luhman et al. 2000). The mass functions thus obtained are extremely interesting as they bear on formation theories, but will not be discussed in this course.

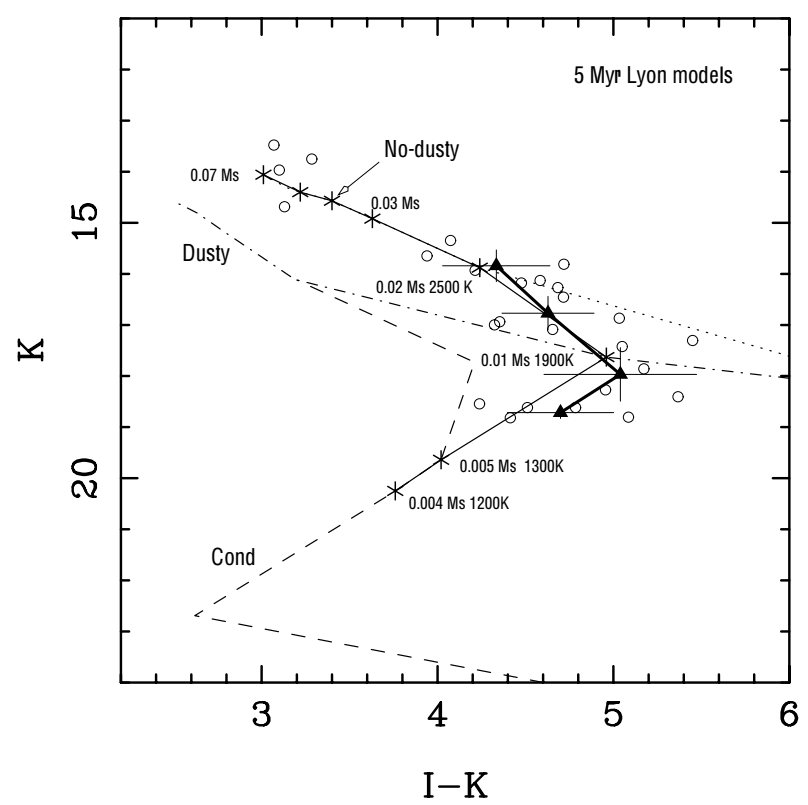

Figure 7.3: $K$ vs $I-K$ color-magnitude diagram. Empty circles are substellar members in $\sigma$ Orionis. The thick solid line and filled triangles represent the mean locus of the $\sigma$ Orionis objects. The 5 Myr isochrones from Chabrier et al. (2000b) are displayed (Nextgen no-dusty models - dotted line, Dusty models - dot-dashed line, and Cond models - dashed line). The thin solid line with asterisks is a best-guess isochrone that combines the models. [From Martín et al. 2001].

Figure 7.3 shows the observations of Martín et al. (2001) in $\sigma$-Orionis as a color-magnitude diagram. The theoretical interpretation using the Lyon model is shown by the dotted, dash-dotted and dashed lines. (The models computed by the Lyon and Tucson groups yield very similar results). As previously, a tendency arise with hotter, more massive objects being closer to a theoretical sequence that accounts for the presence of dust in the atmosphere, and one assuming a clear atmosphere for cooler objects. We will come back to that transition in the following sections. The very low masses obtained for the fainter objects are interesting and one has to consider the uncertainties that are associated with them.

Because the age of the cluster is not precisely known (note that all stars do not form exactly 
at the same time), the intrinsic uncertainty of $1 \sim 5 \mathrm{Myr}$ leads to an uncertainty on the predicted masses. It is interesting to see however that the uncertainty of the models themselves, as computed by two groups and with several assumptions relative to atmospheric composition give strikingly similar results.

First, the age of the cluster is not known. In the case of $\sigma$ Orionis, it is $1 \sim 5 \mathrm{Myr}$. This translates into a factor $\sim 2$ uncertainty on the mass, the young ages corresponding to the lowest values of the masses. Another important source of uncertainty is due to the relative closeness to unknown initial conditions. If in the process of formation the proto-brown dwarfs are able to loose some of their entropy, their interior entropy will be lower than the external one, until cooling (possibly over millions of years) readjusts the profile to a uniform one. For some time the brown dwarf or planet will therefore have a cooler interior, for the same external boundary conditions. (See the entropy profiles and discussion in section 6.2.3). The objects could then be found for some time to the left of the Hayashi track (see fig. 6.6), implying for already compact objects that they could be interpreted to be less massive than they really are. However, the magnitude of this effect has to be investigated.

\subsubsection{Rotation, magnetic activity and variability}

Rotation is a particularly important astrophysical parameter to understand formation processes. In the case of stars, most of the angular momentum present in the molecular cloud had to be lost. Two physical processes can be advocated: the formation of a circumstellar disk and angular momentum transport within that disk, and magnetic braking. The situation is similar for substellar objects.

The rotation rates of brown dwarfs can be inferred through radial-velocity measurements. Contrary to the case of the detection of companions however, one then seeks the intrinsic line broadening due to the rotation. The measured parameter is $v \sin i$, the rotation speed at the equator multiplied by the sine of the inclination. Observations of type M and L stars and brown dwarfs (see e.g. Basri 2000 for a review) indicates that objects with types earlier than $\sim \mathrm{M} 10$ have very widespread values of $v \sin i$, and show intense chromospheric heating, as characterized by their $\mathrm{H} \alpha$ emission. On the other hands, the observed L-type objects are all very fast rotators $(v \sin i=20$ to $80 \mathrm{~km} \mathrm{~s}^{-1}$ ), and have a weak $\mathrm{H} \alpha$ emission. This probably indicates that low-mass objects have a weaker magnetic braking. Several interpretations are possible (see Basri 2000).

Photometric variations of up to $5 \%$ in fluxes have also been detected in $\mathrm{M}$ and $\mathrm{L}$ dwarfs (e.g. Bailer-Jones \& Mundt 2001). In a few cases, these variations are periodic with a period comparable to that of the dwarfs' rotation. They can then be attributed to surface features. Non-periodic variability is also observed, indicating a more complex, time-variable activity. Interestingly, a greater occurrence of variability is found in objects later than M9 indicating that it is not correlated with chromospheric activity. We will see hereafter that in atmospheres at low temperatures (corresponding to dwarfs of later types), condensation sets in. The observed variability could thus well be due to the presence of non-homogeneously distributed, time-variable, photospheric dust clouds.

\subsection{Atmospheric models: importance of condensation}

Modeling the atmospheres of substellar objects is a complex subject that would require several courses. I will focus on a problem which is particular to cool atmospheres: that of condensation. Although condensation has long ago been recognized as an important astrophysical phenomenon, it has been mostly developed in a low- or no-gravity framework. In planetary and substellar 
atmospheres, condensates are expected to be formed, transported, and vaporized continuously, as is the case on Earth. Their study consequently requires new tools, with a particular emphasis on problems related to the transport of material in these atmospheres.

\subsubsection{Basics of condensation}

Let us consider the equilibrium between a condensed phase and a vapor phase of a given chemical species. The thermodynamical condition for equilibrium is that the pressures, temperatures and Gibbs free energies of the two phases should be equal. The last condition implies that:

$$
v^{(\mathrm{v})} d P_{\mathrm{s}}-S^{(\mathrm{v})} d T=v^{(\mathrm{c})} d P_{\mathrm{s}}-S^{(\mathrm{c})} d T,
$$

where the (v) and (c) superscripts indicate the vapor and condensed phases, respectively. $v$ and $S$ are the volume and entropy per unit mass. Eq (7.1) implicitly neglects any surface tension that would appear on a finite size drop formed of condensed material. It is hence valid for equilibrium of the vapor over an infinitely long surface of condensed material. Strictly, the formation of droplets will involve a slightly larger saturation pressure, but this effect will be neglected.

The latent heat is defined as the difference in enthalpies of the two species, hence

$$
L=\left(S^{(\mathrm{v})}-S^{(\mathrm{c})}\right) T .
$$

One therefore obtains from Eq. (7.1) the Clausius-Clapeyron equation:

$$
\frac{d P_{\mathrm{s}}}{d T}=\frac{L}{T\left(v^{(\mathrm{v})}-v^{(\mathrm{c})}\right)} .
$$

Using the perfect gas equation and neglecting the specific volume of the condensed phase over that of the vapor leads to the following equation:

$$
\frac{d \ln P_{\mathrm{s}}}{d \ln T}=\frac{L}{k T} .
$$

This equation allows one, from a known condensation temperature at a given pressure to derive the condensation temperatures at any other pressure. Note that $P_{\mathrm{s}}$ is the saturation pressure, i.e. the pressure corresponding to an equilibrium between the condensate and vapor phases.

The saturation pressure obtained from Eq. (7.4) will be useful in the following for a derivation of the composition gradient, but is only correct to first order. In the case of giant planets and brown dwarfs, the following relations have an accuracy of order $10 \%$ :

$$
\begin{aligned}
\mathrm{H}_{2} \mathrm{O}: & \log _{10} p_{\mathrm{s}}=5.0587-\frac{1630.91}{T-50.396} \\
\mathrm{CH}_{4}: & \log _{10} p_{\mathrm{s}}=4.3180-\frac{451.64}{T-4.66} \quad \text { if } T \leq 91 \mathrm{~K} \\
& \log _{10} p_{\mathrm{s}}=3.8205-\frac{405.42}{T-5.37} \quad \text { if } T \geq 91 \mathrm{~K} \\
\mathrm{NH}_{3}: & \log _{10} p_{\mathrm{s}}=7.0887-\frac{1617.91}{T-0.60} \\
\mathrm{MgSiO}_{3}: & p_{\mathrm{s}}=\exp \left(-\frac{58663}{T}+25.37\right)
\end{aligned}
$$


The pressures are expressed in bars and the temperatures in kelvins. In the case of water, the following approximation for the equilibrium of the vapor with liquid water and ice, respectively, give still better accuracies (better than $0.3 \%$ ) in the temperature interval $-30^{\circ} \mathrm{C} \leq T \leq 40^{\circ} \mathrm{C}$ for liquid water, and $-80^{\circ} \mathrm{C} \leq T \leq 0^{\circ} \mathrm{C}$ for ice:

$$
\begin{aligned}
\text { liquid } \mathrm{H}_{2} \mathrm{O}: & \ln p_{\mathrm{s}}=46.77181-\frac{6743.769}{T}-4.8451 \ln T \\
\text { ice } \mathrm{H}_{2} \mathrm{O}: & \ln p_{\mathrm{s}}=16.42311-\frac{6111.72784}{T}+0.15215 \ln T,
\end{aligned}
$$

where pressures are still in bars and temperatures in kelvin.

\subsubsection{Abundance of condensing species in an atmosphere}

Let us consider an atmosphere in which the condensing species is not the dominant one. The saturation abundance of the condensing species is determined by the ratio of the saturation partial pressure to the total pressure: $x_{\mathrm{s}}=P_{\mathrm{s}} / P$. We introduce the following adimensional quantity:

$$
\beta=\frac{L}{k T} .
$$

For most species of interest in substellar atmospheres, $\beta \approx 10-20$. Assuming that $\beta$ is constant, one can derive the compositional gradient in the atmosphere:

$$
\frac{d \ln x_{\mathrm{s}}}{d \ln P}=\beta \nabla_{T}-1 .
$$

One can thus see that in most cases (e.g. convective tropospheres), $\nabla_{T} \approx 0.3$ and $d \ln x_{\mathrm{s}} / d \ln P \approx$ $2-5$. The abundance at saturation is decreasing with altitude faster than the pressure itself. To the contrary, in nearly isothermal regions, and in stratospheres $\left(\nabla_{T}<0\right)$, the abundance at saturation increases with altitude.

Possible abundance profiles are depicted in Fig. 7.4. In all cases the abundance $x$ has its maximal value (the bulk abundance) and is constant at large depths. However, the composition at upper levels strongly depends on physical mechanisms. In one extreme case (labeled (a) in Fig. 7.4), solid species are immediately removed by gravity and atmospheric circulation is not fast enough to oppose the effect of upward diffusion of the condensing species. The other extreme (b) corresponds to a situation in which solids are transported by convection so rapidly throughout the atmosphere that they can never grow to a size at which they would fall. The total (vapor + solid) abundance is then constant.

In reality, a third situation (c) is more likely. In the presence of advection and sedimentation of part of the condensed material, downward motions will tend to produce an undersaturated mixture, while upward motions will lead to the formation of clouds. This necessarily leads to a non-homogeneous atmosphere, where clouds of the same condensing species appear at various altitudes and do not cover the entire atmosphere. This patchiness is observed in the four giant planets, but is maximal when clouds occur in a convective and not radiative region.

Note that downward motions can lead to a lower-than expected abundance at great depths. This has been observed in Jupiter by the Galileo probe for $\mathrm{NH}_{3}, \mathrm{H}_{2} \mathrm{~S}$ and $\mathrm{H}_{2} \mathrm{O}$ (Niemann et al. 1998), but is also evident in Voyager 2 radio-occultation data for Neptune, in the case of $\mathrm{CH}_{4}$ (Lindal 1992; see also Guillot 1995). 

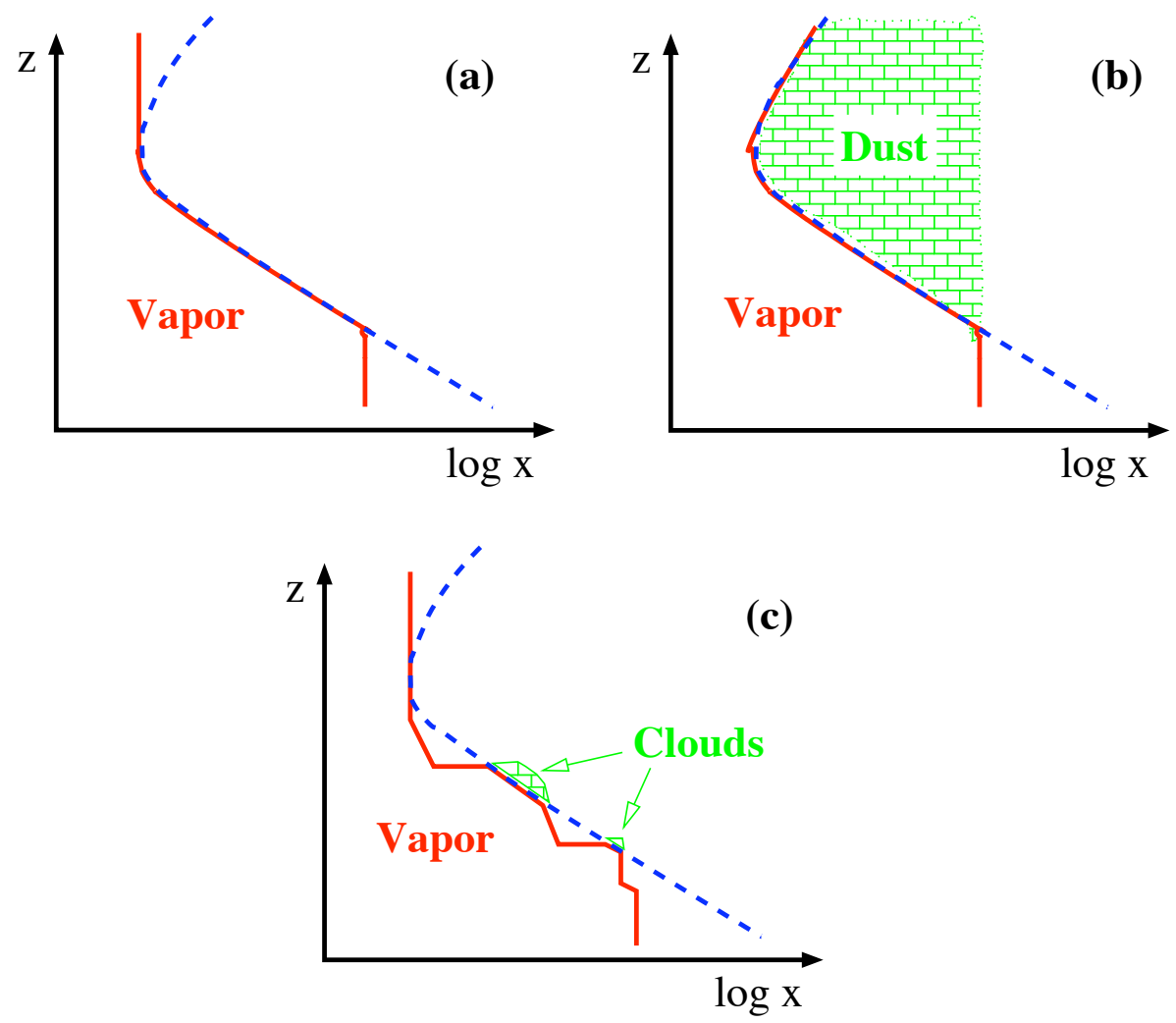

Figure 7.4: Possible abundance profiles $x$ of a condensing species in a substellar or planetary atmosphere (plain lines). The dashed lines corresponds to the saturation profiles. Three cases are: (a) clear atmosphere (immediate "rainout" of condensates); (b) dusty atmosphere (no "rainout"); (c) a possibly more realistic situation (see text).

It is important to stress that depending on the condensation model ( $\mathrm{a}$, b or $\mathrm{c}$ ), the chemistry of the atmosphere will be very different. In model (b), condensed particles are still present in the atmosphere and can react with other species. In model (a) they are completely removed. Therefore, model (a) cannot be consistently calculated by simply using model (b) for the chemistry and removing the opacity arising from condensed particles. In both models (a) and (c), the chemical equilibrium must be consistently calculated, accounting for the full or partial removal of the elements that have condensed.

An important example is titanium: this atom is expected to form $\mathrm{CaTiO}_{3}$ and thus become solid at temperatures $\sim 2000 \mathrm{~K}$. However, chemical equilibrium calculations predict that the abundance of the solid is very small, and that most of the titanium is in form of the strong absorber TiO. If solid $\mathrm{CaTiO}_{3}$ particles are kept in the atmosphere and allowed to react with the environment, TiO will remain as the most important absorber. It is however not observed in Jupiter and Saturn, a sign that condensation, grain growth and subsequent sedimentation have occurred and removed Ti from the upper levels (see e.g. Fegley \& Lodders 1994, 1996). This is also observed in the case of Gl229B, which shows no sign of TiO absorption (Oppenheimer et al. 1998; Marley et al. 1996).

This problem would therefore require to consistently calculate atmospheric models using a 
microphysical description of the clouds and including a description of vertical mixing and a fully self-consistent chemical equilibrium model.

\subsubsection{Temperature profiles}

An important consequence of condensation is to modify heat transport by providing latent heat. The adiabatic temperature gradient is thus modified. Neglecting the heat capacity of condensed species (or equivalently, assuming any condensed material to be left behind during an ascending motion), one can derive the moist pseudo-adiabatic temperature gradient. Using the formulation of Emanuel (1994), but simplified notations:

$$
\nabla_{\text {pseudo }}=\nabla_{\mathrm{ad}}\left[\frac{1+\beta \frac{f}{\epsilon}}{1+\frac{\beta^{2}}{\tilde{c}_{P}} \frac{f}{\epsilon}}\right],
$$

where $\nabla_{\text {ad }}$ is the adiabatic gradient when neglecting latent heat effects (dry adiabatic gradient), $\epsilon=m_{\mathrm{v}} / m_{\mathrm{d}}$ is the ratio between the molecular mass of the condensable species over that of dry air, $f=\rho_{\mathrm{v}} / \rho_{\mathrm{d}}=\epsilon x /(1-x)$ is the mass mixing ratio of the vapor over dry air, and $\tilde{c}_{P}$ is the adimensional mean specific heat per molecule (including dry air and vapor).

In the cases that are of interest to us, $f / \epsilon \ll 1$ so that

$$
\nabla_{\text {pseudo }} \approx \nabla_{\text {ad }}\left[1-\left(\frac{\beta}{\tilde{c}_{P}}-1\right) \beta \frac{f}{\epsilon}\right] .
$$

Because $\beta \approx 10-20$ and $\tilde{c}_{P} \approx 3.5$, we obtain that $f / \epsilon \approx x \gtrsim 2 \times 10^{-4}$ in order to change the adiabatic gradient by $\sim 1 \%$ or more. In objects of approximately solar composition, the only potentially condensable species that are abundant enough are $\mathrm{H}_{2} \mathrm{O}$ and $\mathrm{CH}_{4}$. Note that $\mathrm{NH}_{3}$ can induce a change of $\sim 0.6 \%$, and compounds formed from $\mathrm{Mg}, \mathrm{Si}$ and Fe a change of $\sim 0.2 \%$.

The phenomenon of moist convection, i.e. of convection powered by latent heat release, such as that observed in cumulus clouds on Earth is therefore likely to be limited to atmospheres in which water and methane can condense, i.e. to relatively cold atmospheres. In the case of condensation of more refractory species, the limited effect of latent heat release on the temperature gradient is likely to be outweighted by the strongly inhibiting condensate loading.

Another consequence of condensation in substellar hydrogen atmospheres is that it yields a stable molecular weight gradient. Assuming that the atmosphere is saturated and using Eq. (7.13),

$$
\nabla_{\mu}=\varpi f\left(\beta \nabla_{T}-1\right),
$$

where $\varpi=(1-1 / \epsilon) /(1+f)$. In the case of hydrogen atmospheres, $\varpi \sim 1, \beta \sim 20, \nabla_{T} \sim 0.3$ so that $\nabla_{\mu}>0$. In the case of the Earth, $\varpi \sim-0.5$ due to the smaller weight of the water molecule than of $\mathrm{N}_{2}$ : in that case, moist air tends to rise, thereby favoring the occurrence of moist convection.

In the ideal case of a saturated atmosphere in which condensed species are removed instantaneously by gravity, the criterion for convective is slightly modified compared to Eq. (4.19). Because of condensation occurring both in the environment and the upwelling parcel, the local criterion becomes (Guillot 1995):

$$
(1-\varpi \beta f)\left(\nabla_{T}-\nabla_{\text {pseudo }}\right)>0 .
$$

Convection is thus inhibited when the abundance of the condensable is such that

$$
f>(\varpi \beta)^{-1} \text {. }
$$


Physically, this condition results from the fact that the abundance of condensable species drops faster in the environment than in the rising parcel. In spite of its higher temperature, the parcel thus becomes negatively buoyant. This occurs however only for condensing species whose mass mixing ratio can raise above $\sim 0.03$, i.e. enrichment over the solar value of $\sim 5$ for $\mathrm{H}_{2} \mathrm{O}, \sim 15$ for $\mathrm{CH}_{4}$ and $\sim 10-20$ in silicates and iron. This is potentially interesting in the case of water condensation in Jupiter and Saturn and of methane condensation in Uranus and Neptune. In the case of the two latter planets, it could explain the superadiabatic gradients obtained from the Voyager radio-occultation in the region of methane condensation (Guillot 1995).

For objects of solar composition however, the molecular weight gradient effect is limited, i.e. $\nabla_{\mu} \lesssim 10^{-2}$ as long as water condensation is not involved. To first order, the effect of condensation on the temperature gradient can be neglected.

Figure 7.5 shows several atmospheric temperature profiles calculated by Burrows et al. (1997) for isolated substellar objects. The figure shows as dashed lines the limits for condensation of water, ammonia, $\mathrm{MgSiO}_{3}$ and iron, assuming solar composition. The lines where $\mathrm{CO}$ and $\mathrm{CH}_{4}$ and where $\mathrm{N}_{2}$ and $\mathrm{NH}_{3}$ have the same abundances are also indicated. As can be intuited, depending on the effective temperatures and gravity, various elements are expected to condense near the photospheres of substellar objects having great effects on their spectra: Particularly important are the condensation of methane, ammonia and water for low effective temperatures, and iron and silicates in relatively warm atmospheres. Note that these are only the most abundant species to be formed: other potentially condensing species include numerous sulfides and chlorides (e.g. $\mathrm{K}_{2} \mathrm{~S}$, $\mathrm{Na}_{2} \mathrm{~S}$...etc.)

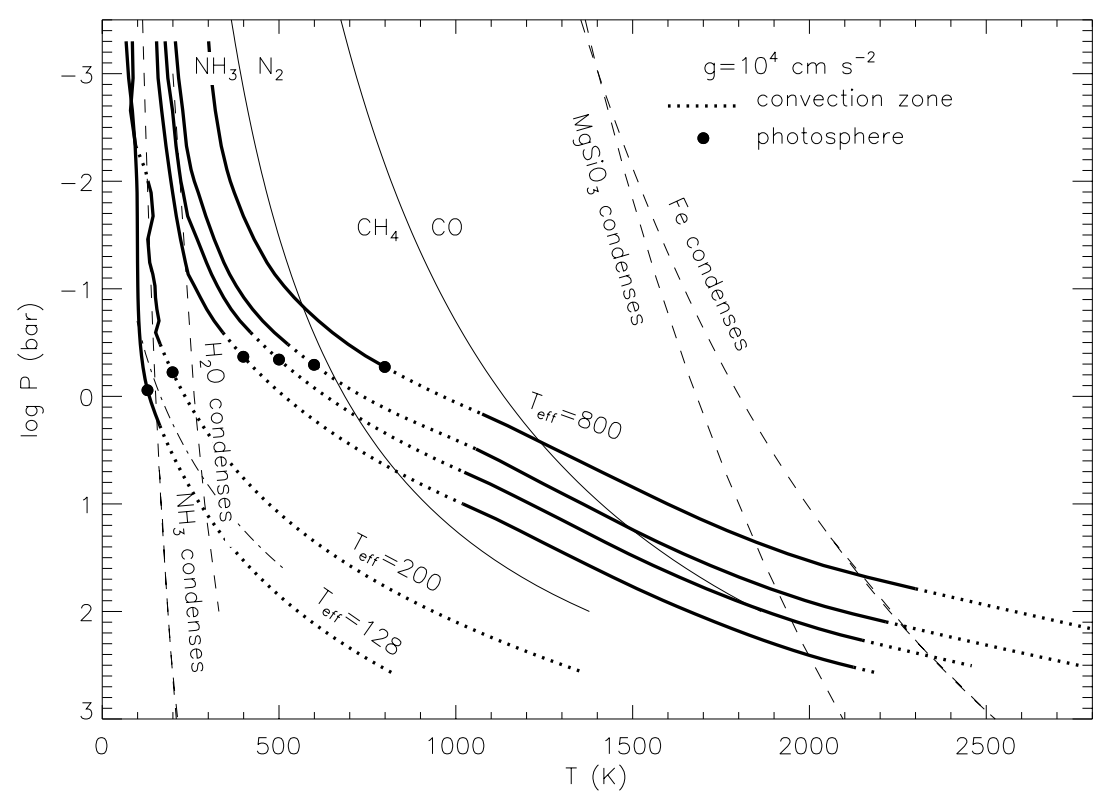

Figure 7.5: Atmospheric pressure-temperature profiles for non-irradiated substellar atmospheres with surface gravity fixed at $10^{4} \mathrm{~cm} \mathrm{~s}^{-2}$ and $\mathrm{T}_{\text {eff }}=800,600,500,400,200$, and $128 \mathrm{~K}$. Note that the inner radiative zones disappear in the presence of alkali metals (not accounted for in the calculation). [From Burrows et al. (1997)] 
The temperatures profiles of Fig. 7.5 do not account for the presence of any clouds. When present, these would lead to a significant warming of the atmosphere (see Allard et al. 2001). Note that the inner radiative zones shown in Fig. 7.5 disappear when alkali metals are included in the calculation.

\subsubsection{Dust and clouds: relevant physical processes}

The formation of dust and clouds in substellar atmospheres is governed by several processes:

1. Chemistry: given the composition, temperatures and pressures of a substellar atmosphere, a set of chemical species is predicted to undergo vapor/liquid or vapor/solid phase changes. The deepest level at which that occurs is the condensation level, sometime improperly called cloud base (as we can see from model (c) in Fig. 7.4, clouds do not necessarily originate from that level). Among the different kinds of condensation, one might distinguish the condensation of a minor species, as it involves a chemical reaction (e.g. $\mathrm{CaTiO}_{3}$ with $\mathrm{TiO}$ remaining in gaseous form), and the condensation of a major species (e.g. $\mathrm{H}_{2} \mathrm{O}$ ).

2. Grain growth: this groups all the mechanisms that affect the size of the condensed grains or droplets. Those include condensation (vapor molecules/atoms sticking upon an already condensed site), coagulation (due to Brownian motion) and coalescence (merging of big droplets with slightly different vertical velocities). It also includes evaporation which occurs in an undersaturated environment (i.e. when the partial pressure of the condensing species is smaller than its saturation pressure).

3. Sedimentation: condensed particles are affected by gravity forces, the more massive ones falling more rapidly than the lighter ones.

4. Mixing: the advection of saturated/undersaturated gas and small particles due to various effects (convective instability, meridional circulation, waves...etc.) inevitably influences grain growth and sedimentation. As we have seen, in the case of water and methane, this is complicated by the significant latent heat effect that tends to favor updrafts in which condensation occurs.

5. Radiative heating/cooling: the presence of solid/liquid particles modifies the radiative properties of the medium, which can in turn affect mixing (by creating small-scale or large-scale instabilities) and condensation (by modifying the temperature profiles).

The processes of grain growth and sedimentation can be approximated using the timescales provided by Rossow (1978). These estimates are applied to the case of an iron cloud in a typical $2000 \mathrm{~K}$ brown dwarf and shown in fig. 7.6 (see also Lunine et al. 1989). Grain growth is dominated by condensation for sizes larger than several microns. However, before they reach those sizes, they are expected to be removed efficiently by sedimentation. Let us define a time $\tau^{\star}$ and size $a^{\star}$ as satisfying the following condition:

$$
\tau_{\text {sedimentation }}\left(a^{\star}\right)=\tau_{\text {growth }}\left(a^{\star}\right) \equiv \tau^{\star} .
$$

These two quantities are defined by the point at which the sedimentation and condensation lines cross in fig. 7.6.

The amount of condensed particles and their sizes will then depend on the mixing of vapor and small particles from levels above and below the one considered. In terrestrial clouds, this is 


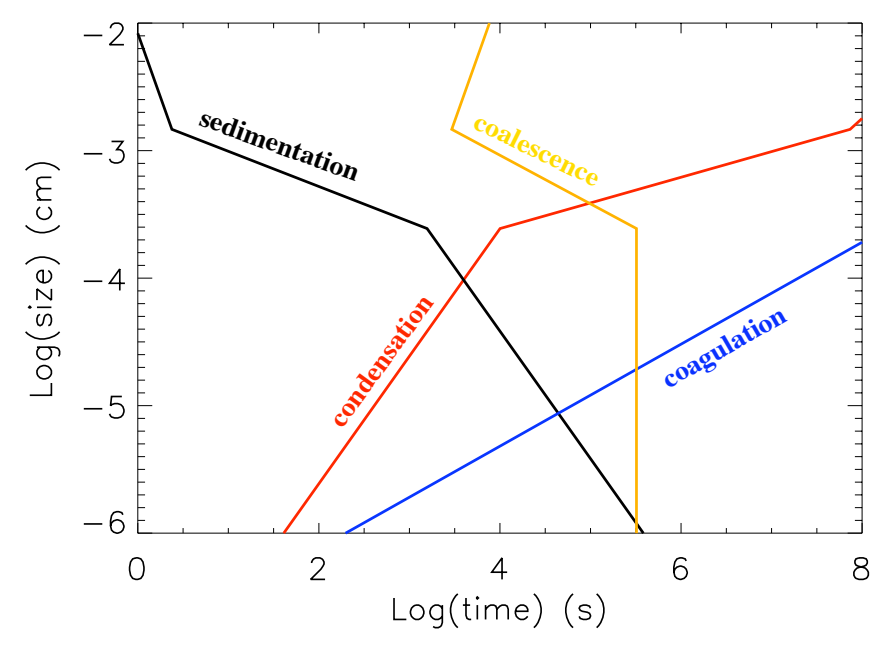

Figure 7.6: Time scales for grain growth and sedimentation at the basis of an "iron-cloud" in a brown dwarf of $T_{\text {eff }}=2000 \mathrm{~K}, g=3 \times 10^{5} \mathrm{~cm} \mathrm{~s}^{-2}$. [Brown dwarf model: courtesy of F. Allard; timescales from Rossow 1978].

modeled through the solution of a complex set of differential equations (see e.g. Cotton \& Anthes 1989). In giant planets and substellar objects this problem is far from being well understood, and a much simpler approach is generally sufficient. One thus generally defines a turbulent diffusivity as relevant of mixing processes in the atmosphere. In our very simple case, we will define $\tau_{\text {mix }}$ as the characteristic time scale for mixing over one pressure scale height $H_{P}$.

Two cases occur:

1. $\tau_{\text {mix }} \lesssim \tau^{\star}$ : This is the case of efficient mixing (i.e. case (b) in fig. 7.4). Fresh particles and vapor are constantly supplied by the mixing before they can fall under the action of gravity. In the limit when mixing does not affect the largest grains, the final mean size is expected to be of the order of $a^{\star}$. If the largest grains are also transported, then the final mean radius will depend mostly on the time during which they remain above the condensation level. The situation can become complex: in the Earth's clouds, a bi-modal distribution of water droplets is generally observed (e.g. Cotton \& Anthes 1989).

2. $\tau_{\text {mix }} \gtrsim \tau^{\star}$ : Here, the relatively sluggish mixing prevents the formation of particles as large as $a^{\star}$ because they are removed by sedimentation. The mean size is expected to be largely set by the equilibrium between mixing and sedimentation, i.e. $\tau_{\text {mix }}=\tau_{\text {sedimentation }}$. This yields of course particles of sizes smaller than $a^{\star}$. It also yields a lower abundance of particles (i.e. case (a) or (c) in fig. 7.4).

\subsubsection{A transition from dusty to clear atmospheres}

As shown in fig. 7.2, there is a transition from high effective temperature, red, and dusty brown dwarfs, to brown dwarfs of lower effective temperatures that are much bluer and appear to have clear atmospheres (or more accurately, to deviate from case (b) of fig. 7.4). This transition is 
thought to be due to the sedimentation of dust in brown dwarfs of low temperatures and also to the additional cooling due to the $\mathrm{CO}$ to $\mathrm{CH}_{4}$ transition.

Let us focus first on the high temperature, dusty brown dwarfs. In the case of our $T_{\text {eff }}=2000 \mathrm{~K}$ brown dwarf, one finds that $a^{\star} \approx 1 \mu \mathrm{m}$, and $\tau^{\star} \approx 3 \times 10^{3} \mathrm{~s}$. The time scale for mixing in the convective zone can be estimated from $\tau_{\text {mix }} \approx H_{P} / v$ and $v$ is the convective velocity from eq. (4.34). At the basis of the iron cloud, we have $T \approx 1700 \mathrm{~K}$ and $P \approx 0.4$ bar. The convective velocity would then be $v \approx 2 \times 10^{4} \mathrm{~cm} \mathrm{~s}^{-1}, H_{P} \approx 2 \times 10^{5} \mathrm{~cm}$ and hence $\tau_{\operatorname{mix}} \approx 10 \mathrm{~s}$. However, convection is found to start at much deeper levels, i.e. around 10 bar (F. Allard, pers. communication). The good results obtained from stellar models using an ad hoc interstellar grain size distribution (Allard et al. 2001) indicates that the mean particle size is indeed probably between 0.1 and $1 \mu \mathrm{m}$. Therefore, a mixing process is needed to explain the presence of these particles in the brown dwarfs upper photosphere. Several possibilities exist, and the fact that these objects are generally fast rotators (e.g. Basri 2000) is interesting because it could yield enough meridional circulation to provide the right amount of mixing.

The transition to brown dwarfs of lower temperatures ("T-dwarfs") is still unclear. A possible model based on the timescales discussed here is provided by Ackerman \& Marley (2001), and with a free parameter reproduces relatively well the observations. However, the model assumes an eddy mixing time scale that even in radiative regions is arbitrarily large. One may therefore wonder whether the real problem to solve may instead be "why are grains present in brown dwarfs of high effective temperatures?".

Observational constraints on the amount of atmospheric mixing exist at least for one wellstudied brown dwarf: Gl229B. The detection of chemical species that are out of thermochemical equilibrium informs us on how fast these species are transported throughout the atmosphere. This is in particular the case of $\mathrm{CO}$ which partially escapes a transformation into $\mathrm{CH}_{4}$ as it is transported upward in Gl229B's atmosphere (roughly from levels of $\sim 10$ bar to $\sim 1$ bar where it is detected). Griffith \& Yelle (1999) estimate that the diffusion coefficient of mixing is $K_{\operatorname{mix}} \sim 3 \times 10^{2}$ to $10^{4} \mathrm{~cm}^{2} \mathrm{~s}-1$. The mixing time scale is (very imprecisely!) $\tau_{\operatorname{mix}} \approx H_{P}^{2} / K_{\operatorname{mix}} \approx 1.5 \times 10^{6}$ to $5 \times 10^{9} \mathrm{~s}$. This is to be compared to the mixing times of $10-10^{3} \mathrm{~s}$ required to keep grains up in the atmosphere.

\subsubsection{Dust and variability}

The presence of dust opens new possibilities for atmospheric variability. As discussed previously, moist convection is not a likely possibility in the case of brown dwarfs and hot giant planets. The situation is therefore different than for our giant planets. However, variability is linked to the spatial heterogeneities. The fact that in Jupiter, small regions of the planet can emit much more than others because of a lack of clouds there has to be kept in mind.

One possibility for the presence of Jupiter's hot spots is the presence of a planetary wave (Showman \& Dowling 1998). In the same frame of mind, waves could well affect the distribution of dust in the atmosphere of brown dwarfs. A potential interesting source of waves is again in the rapid rotation of these objects and the possibility of Rossby and Kelvin waves. Baroclinic instabilities linked to the rotation and the presence of meridional circulation are also a possibility. Finally, a coupling between dust formation and heat transfer may be envisioned: we have seen that the presence of dust indeed greatly increases the opacity. 


\section{Chapter 8}

\section{Pegasi Planets ("51 Peg b-like" Planets)}

\subsection{Introduction}

The detection of planetary-mass companions in small orbits around solar-type stars has been a major discovery of the past decade. To date, 73 extrasolar giant planets (with masses $M \sin i<$ $13 \mathrm{M}_{\mathrm{J}}, i$ being the inclination of the system) have been detected by radial velocimetry. Fifteen of these $(21 \%)$ have distances less than $0.1 \mathrm{AU}$, and ten (14\%) have distances less than $0.06 \mathrm{AU}$ (see Marcy et al. 2000 and the discoverers' web pages). This is for example the case with the first extrasolar giant planet to have been discovered, 51 Peg b (Mayor \& Queloz 1995). These close-in planets form a statistically distinct population: all planets with semi-major axis smaller than 0.06 AU have near-circular orbits while the mean eccentricity of the global population is $\langle e\rangle \approx 0.27$. This is explained by the circularization by tides raised on the star by the planet (Marcy et al. 1997). One exception to this rule, HD83443b $(e=0.079 \pm 0.033)$, can be attributed to the presence of another eccentric planet in the system (Mayor et al. 2001). As we shall see, the planets inside $\sim 0.1$ $\mathrm{AU}$ also have very specific properties due to the closeness to their star and the intense radiation they receive. For this reason, following astronomical conventions, I choose to name them after the first object of this class to have been discovered: "51 Peg b-like" planets, or in short "Pegasi planets".

Such planets provide an unprecedented opportunity to study how intense stellar irradiation affects the evolution and atmospheric circulation of a giant planet. Roughly $1 \%$ of stars surveyed so far bear Pegasi planets in orbit, suggesting that they are not a rare phenomenon. Their proximity to their stars increases the likelihood that they will transit their stars as viewed from Earth, allowing a precise determination of their radii. (The probability varies inversely with the planet's orbital radius, reaching $\sim 10 \%$ for a planet at $0.05 \mathrm{AU}$ around a solar-type star.) One planet, HD209458b, has already been observed to transit its star every 3.524 days (Charbonneau et al. 2000; Henry et al. 2000). The object's mass is $0.69 \pm 0.05 \mathrm{M}_{\mathrm{J}}$. Hubble Space Telescope measurements of the transit (Brown et al. 2001a) imply that the planet's radius is $96300 \pm 4000 \mathrm{~km}$ (see light curve on fig. 8.1). An analysis of the lightcurve combined with atmospheric models shows that this should correspond to a radius of $94430 \mathrm{~km}$ at the 1 bar level (Hubbard et al. 2001). This last estimate corresponds to $1.349 \overline{\mathrm{R}}_{\mathrm{J}}$, where $\overline{\mathrm{R}}_{\mathrm{J}} \equiv 70,000 \mathrm{~km}$ is a characteristic radius of Jupiter. This large radius, in fair agreement with theoretical predictions (Guillot et al. 1996), shows unambiguously 


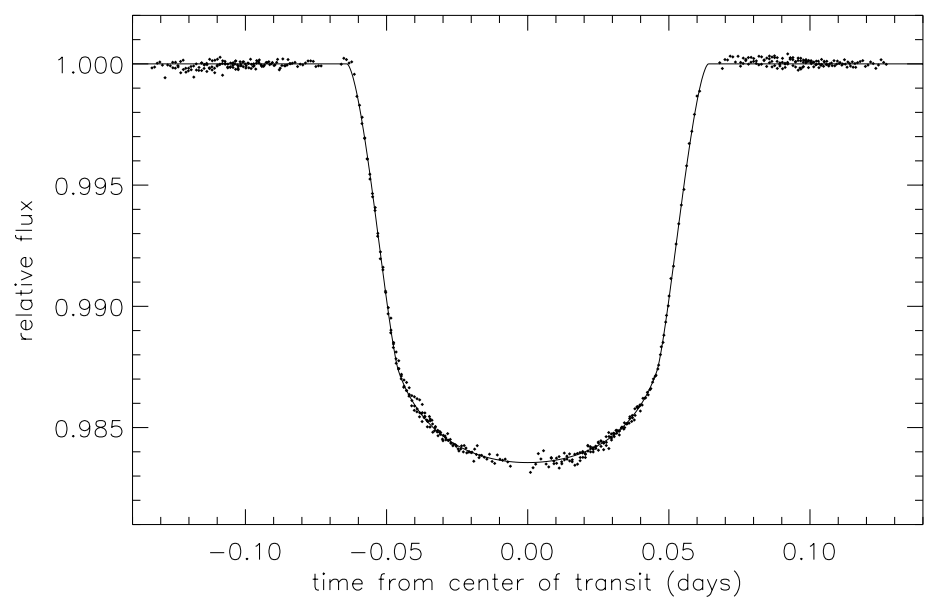

Figure 8.1: Phased light curve of four planetary transits across the star HD209458 observed with the HST. The orbital period of the planet is 3.52474 days. [Figure from Brown et al. (2001a)]

that HD209458b is a gas giant.

One expects that the evolution of Pegasi planets depends more on the stellar irradiation than is the case with Jupiter. HD209458b and other Pegasi planets differ qualitatively from Jupiter because the globally-averaged stellar flux they absorb is $\sim 10^{8} \mathrm{erg} \mathrm{cm}^{-2}\left(10^{5} \mathrm{~W} \mathrm{~m}^{-2}\right)$, which is $\sim 10^{4}$ times greater than the predicted intrinsic flux of $\sim 10^{4} \mathrm{erg} \mathrm{cm}^{-2}$. (In contrast, Jupiter's absorbed and intrinsic fluxes are the same within a factor of two.)

\subsection{Evolution of strongly irradiated giant planets}

\subsubsection{Including stellar heating: definitions}

As discussed in section 6.2.2, the total luminosity of an irradiated planet or brown dwarf consists of three parts: (i) the part of the stellar flux which is directly reflected and does not contribute to the atmosphere's energy budget; (ii) $L_{\mathbf{o}}$, the part which is absorbed, thermalized and reemitted (we assume that no seasonal effects take place and that the system is in equilibrium); (iii) $L$, the intrinsic luminosity due to the object's contraction and cooling.

First, the effective temperature of any irradiated planet is defined by:

$$
4 \pi R^{2} \sigma T_{\text {eff }}^{4}=L+L_{\mathbf{o}}
$$

and the equilibrium effective temperature $T_{\mathbf{0}}$ by

$$
4 \pi R^{2} \sigma T_{\mathbf{\bullet}}^{4}=L_{\mathbf{o}}
$$

$T_{\mathbf{\sigma}}$ is the effective temperature toward which the planet tends as it cools and $L \rightarrow 0$. It is a function of the Bond albedo $A$ (i.e. the ratio of the luminosity directly reflected to the total luminosity intercepted by the planet):

$$
T_{\mathbf{\sigma}}=T_{\star}\left(\frac{R_{\star}}{2 D}\right)^{1 / 2}(1-A)^{1 / 4}
$$


where $T_{\star}$ and $R_{\star}$ are respectively the star's effective temperature and radius, and $D$ is the starplanet distance.

It is important to stress that these definitions are valid independently of heat absorption and heat transport in the atmospheres of these objects. Most of the physics is hidden in the Bond albedo $A$. The values for our giant planets are listed in table 1.3. They all lie between 0.29 (Neptune) and 0.35 (Jupiter). In the case of extrasolar giant planets, simulations indicate similar values of the albedo when alkali metals are not present (Marley et al. 1999), but very low values $A \sim 0.05$ when alkali metals contribute to the absorption in the optical (Sudarsky et al. 2000). This would imply that extrasolar planets are very difficult to detect in the optical, since they reflect little of the incoming flux. However, the albedo can be significantly modified by the presence of grains in the atmosphere (see Marley et al. 1999). Our understanding of grain and cloud formation being far from complete, these estimates of $A$ have to be taken with caution.

Finally, in the case of Pegasi planets we will see that the stellar heat is absorbed very inhomogeneously and is not necessarily well redistributed over the entire atmosphere. This does not affect the above definitions, but it strongly modifies any calculation of the atmospheric structure. The bond albedo, and surface boundary conditions then have to account for this. I will focus on cases in which this effect is neglected. However, the effect of day/night temperatures variations both on the evolution and on chemistry in Pegasi-planet atmospheres will be discussed.

\subsubsection{Temperature of irradiated atmospheres}

Most of the course has been concerned with mostly-convective objects. We have seen in section 6.2.2 that in this case, which corresponds to weak irradiation, a relatively trivial modification of the external boundary condition was sufficient to obtain a relatively good estimate of the evolution. We derived (Eq. (6.20)) an evolution time scale for weakly irradiated object that was equal to the time scale in isolation plus an expansion in powers of $\left(T_{\mathbf{o}} / T_{\text {eff }}\right)^{4}$. In the case of Pegasi planets however, the strong stellar irradiation leads to $T_{\text {eff }} \approx T_{\mathbf{o}}$ (the absorbed stellar flux is typically about $10^{4}$ times stronger than the intrinsic heat flux). With the assumptions of section 6.2 .2 , one would find a cooling time scale tending to infinity. This is because when $T_{\text {eff }} \rightarrow T_{\mathbf{0}}, L \rightarrow 0$, and the planetary interior necessarily becomes partly radiative.

The strong irradiation thus not only significantly slows the cooling of the planet, it also profoundly modifies its very structure. The growth of a radiative zone located just below the "atmosphere" (defined as the region which is penetrated by the stellar photons) implies that standard boundary conditions cannot be used. The problem hence becomes relatively complex, and requires a detailed treatment of the radiative transfer equations in the atmosphere.

In the absence of adequate atmospheric models, Guillot et al. (1996) however derived evolution models for Pegasi planets using the approximation of section 6.2.2. This was also later used by Burrows et al. (2000b) for the evolution of HD209458b. In these papers, the atmospheric boundary condition is at the same pressure and temperature than that of an isolated object of the same effective temperature:

$$
T(P=10 \text { bars })=T_{\text {isolated }}\left(T_{\text {eff }}, g\right)
$$

This approximation is exact in the limit when the stellar luminosity is entirely absorbed at the 10 bar level, or if the region of absorption is connected to the 10 bar level by an isentrope (i.e. the 10 bar level is in a nearly-adiabatic convective zone).

Unfortunately, the approximation becomes incorrect in the case of strongly irradiated planets because of the growth of a thick external radiative zone. Another boundary condition has therefore to be sought: either part of the stellar flux is able to penetrate to deeper levels $\left(P_{0}>10\right.$ bar $)$ 
and lead to a boundary condition defined by $T\left(P_{0}\right)>T_{\text {isolated }}$, or most of the stellar flux is absorbed at $P_{0}<10$ bar, yielding $T\left(P_{0}\right)<T_{\text {isolated }}$. (This is due to the fact that in the radiative zone $d T / d P \propto F$, where $F$ is the flux to be transported). Indeed, more detailed models of the atmospheres of Pegasi planets have shown that most of the starlight is absorbed at pressures less than 10 bar, and that Eq. (8.4) overestimates the atmospheric temperatures by as much as 300 to 1000 K (Seager \& Sasselov 1998, 2000; Goukenleuque et al. 2000; Barman et al. 2001).

A similarly incorrect approach has been used by Lin, Bodenheimer \& Richardson (1996) and Bodenheimer et al. (2001): they also use the same approximation as described in section 6.2 .2 , but instead of Eq. 8.4, they use the Eddington approximation (Eq. (2.5)). Their boundary pressure $P_{0}$ is much smaller, i.e. $P_{0} \approx 1 \mathrm{mbar}$, a consequence of $g \approx 10^{3} \mathrm{~cm} \mathrm{~s}^{-2}$ and $\kappa \approx 1 \mathrm{~cm}^{2} \mathrm{~g}^{-1}$. Their temperatures in the deep atmospheres (around 10 bar) are much smaller than calculated by detailed atmospheric models. This is mostly due to the fact that the opacities used (Pollack et al. 1994) are very high because they include the presence of grains but no sedimentation. We have seen in section 7.2.2 that the true abundance of grains should be much lower. With $\kappa \approx 10^{-3} \mathrm{~cm}^{2} \mathrm{~g}^{-1}$, a more realistic value, the photospheric pressure becomes $P_{0} \approx 1$ bar. However, a more serious problem is that the Eddington approximation has no theoretical validity for irradiated atmospheres.

In order to get around that problem Guillot \& Showman (2002) use an ad hoc atmospheric boundary temperature which is lower than predicted by Eq. (8.4) by $1000 \mathrm{~K}$. According to the detailed atmospheric models, this "cold" boundary condition is supposed to be more realistic.

\subsubsection{Hertzprung-Russell diagram}

On a Hertzprung-Russell diagram ( $L$ vs. $T_{\text {eff }}$ ), isolated brown dwarfs and planets essentially follow the Hayashi evolution track, which is defined by the trajectory of a fully convective object of given mass and composition (Hayashi 1961). Figure 6.6 shows a set of Hayashi lines for various masses. Massive brown dwarfs can only slightly deviate from these tracks in the case of the growth of an inner conductive region. This is however a relatively marginal effect. In the case of strongly irradiated planets, the deviation from the Hayashi track can be much more pronounced.

Figure 8.2 is a H-R diagram for irradiated $1-\mathrm{Mj}$ planets. The dark region at low effective temperatures (to the right-hand side of the graph) corresponds to the Hayashi forbidden region: no object in hydrostatic equilibrium can be found there because it would violate the Schwarzschild instability criterion. (The situation is more complex in the presence of stabilizing compositional gradients, but this effect will be ignored). The forbidden region is bounded to the left by the fully-convective Hayashi line.

Jupiter is found to follow the Hayashi line (even when a radiative zone such as that found by Guillot et al. (1994a) is included, the departure is small). The evolution starts with an extended planet, to the top right of the diagram. In a first phase, the contraction yields higher values of $T_{\text {eff }}$. When the degeneracy becomes important, the virial theorem shows that the luminosity is then essentially provided by the planet's cooling and the effective temperature decreases (see section 6.1).

In the case of irradiated planets, the evolution cannot proceed to low values of $T_{\text {eff }}$ due to eq. (8.1). It is easy to see that the planet then cannot stay on the Hayashi line. Not only would it imply an nonphysical singularity in the diagram, it would also violate thermodynamics principles: the planet would then stop cooling, but its interior would remain much hotter than its atmosphere $(\sim 30,000 \mathrm{~K}$ vs. $\sim 1500 \mathrm{~K})$.

The solution to this paradox is, as discussed previously, the growth of an inner radiative zone. The evolution tracks are then observed to deviate substantially from the Hayashi line, as shown by 


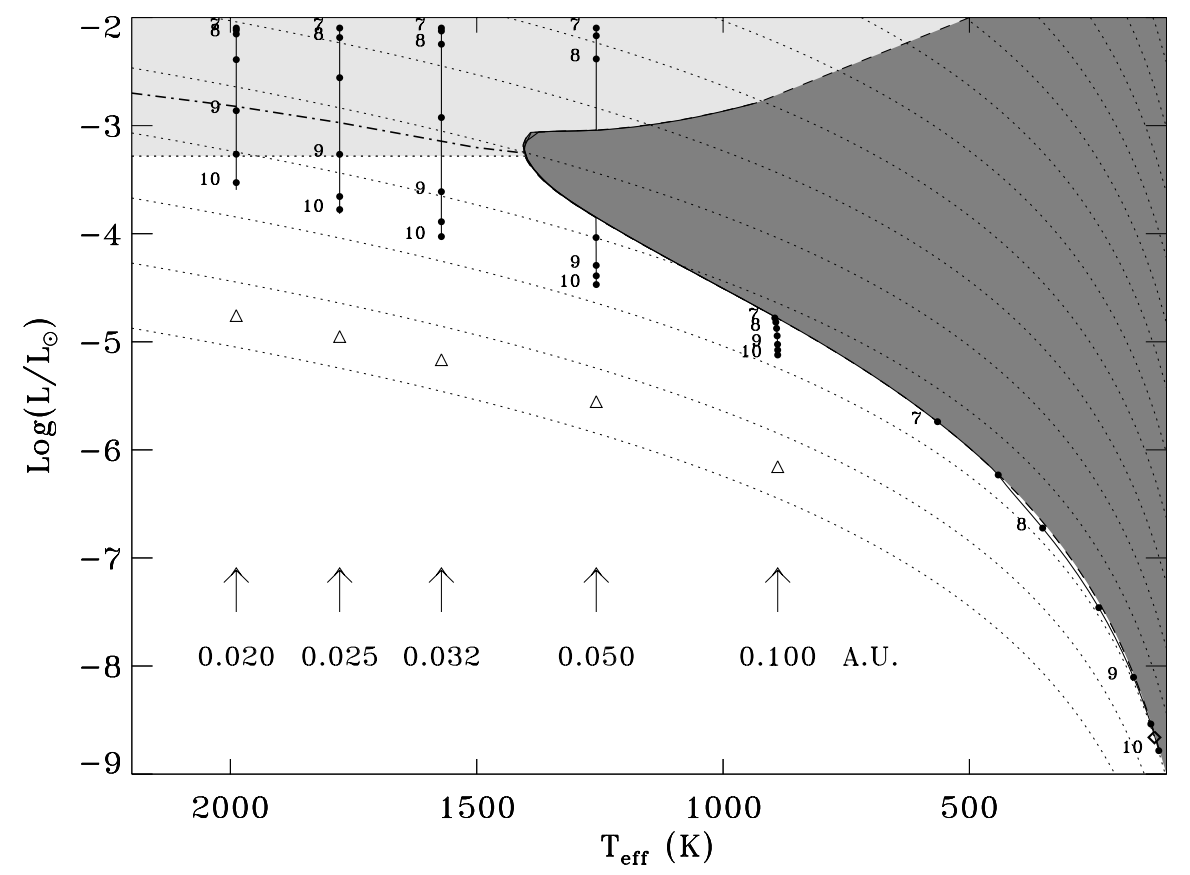

Figure 8.2: Hertzprung-Russell diagram for $1 \mathrm{M}_{\mathrm{J}}$ planets orbiting at $0.02,0.025,0.032,0.05$ and $0.1 \mathrm{AU}$ from a solar-type star, assuming a Bond albedo of 0.35. Arrows indicate the corresponding equilibrium effective temperature $\left(T_{\mathbf{o}}\right)$. A Jupiter model is also shown, the diamond in the bottom right-hand corner corresponding to the present-day effective temperature and luminosity of the planet. Evolutionary tracks for planets of solar composition are indicated by lines connecting dots which are equally spaced in $\log$ (time). The numbers $7,8,9$ and 10 are the common logarithms of the planet's age. Zero-temperature models for $1 \mathrm{M}_{\mathrm{J}}$ planets made of olivine $\left(\mathrm{Mg}_{2} \mathrm{SiO}_{4}\right)$ are indicated by triangles. The Hayashi forbidden region, which is enclosed by the evolutionary track of the fully convective model, is shown in dark gray (see text). Models in the light gray region have radii above the Roche limit (and therefore are tidally disrupted by the star). The region where classical Jeans escape becomes significant is bounded by the dash-dotted line. Lines of constant radius are indicated by dotted curves. These correspond, from bottom to top, to radii (in units of $\overline{\mathrm{R}}_{\mathrm{J}}$ ) in multiples of 2, starting at $1 / 4$. [From Guillot et al. (1996)] 
the almost vertical lines in fig. 8.2. A very important consequence of the growth of the radiative zone is that the planets continue to shrink, and their radii after a few billions of years of evolution are close to that of Jupiter, assuming a solar composition. (In the case of planets made entirely out of rocks, the radii obtained are about $1 / 3 \overline{\mathrm{R}}_{\mathrm{J}}$, as indicated by triangles in fig. 8.2.)

The evolution is found to be relatively fast on the Hayashi line, but slow away from it. This is due to the fact that when a radiative zone develops, due to irradiation, the ability of the planet to cool is not governed by the heat leaking from the atmosphere but instead by the slow shrinking or growth of the radiative zone. In the early phases, when the irradiated planet is very extended, the radiative zone shrinks as the object contracts. This slow evolution is effectively a problem for the formation of the planet at this distance. One can get around this problem by increasing the abundance of solids in the nebula and progressively capturing the gaseous envelope (e.g. Bodenheimer et al. 2000). However, a more simple (probable?) solution is that the planets were not formed very close to their stars but were formed at greater distances and then migrated inward (Lin et al. 1996; see also Trilling et al. 1998 and the chapter by Pat Cassen).

\subsubsection{Growth of a radiative zone}

The evolution models applied to HD209458b $\left(M=0.69 \mathrm{M}_{\mathrm{J}} ; T_{\mathbf{a}}=1400 \mathrm{~K}\right)$ are shown in fig. 8.3. Initially ( $t \lesssim 10^{7}$ years in this case), as predicted by the virial theorem, the interior heats up. When the central pressure rises above several Mbar, the gravitational energy lost due to the contraction is mostly used to increase the pressure of the degenerate electrons. The luminosity is then mostly due to the planet's cooling.

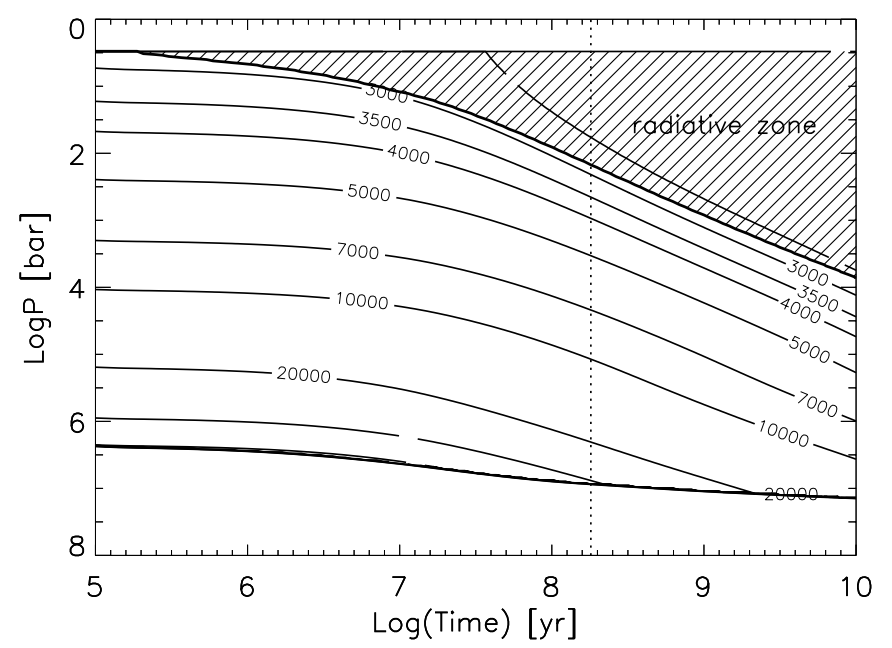

Figure 8.3: Evolution of HD209458b using a "cold" atmospheric boundary condition (see text). The evolution of the central pressure with time is shown as the bottom thick line. The planet is convective except for an upper radiative zone indicated by a hashed area. Isotherms from 4000 to $20000 \mathrm{~K}$ are indicated. The isotherms not labeled correspond to 3500,30000 and $40000 \mathrm{~K}$. The dashed line indicates the time necessary to contract the planet to a radius of $1.35 \overline{\mathrm{R}}_{\mathrm{J}}$. [From Guillot \& Showman (2002)] 
As discussed previously, due to the strong stellar irradiation, the only possibility for the interior to cool is through the growth of a radiative zone, as evidenced by the dashed region in fig. 8.3. This region is almost isothermal. In these calculations, Guillot \& Showman (2002) used opacities from Alexander \& Ferguson (1994). These opacities do not include the presence of alkali metals, but they include the presence of grains. Other opacity tables including alkali metals and grain settling should be used, but are expected not to significantly alter the results.

\subsubsection{Reproducing the radius of HD209458b}

Of course, an evident constraint to the model calculations is the photometrically-measured radius of HD209458b. Figure 8.4 shows how the radius of a $0.69 \mathrm{M}_{\mathrm{J}}$ solar-composition object with $T_{\mathbf{\sigma}}=$ $1400 \mathrm{~K}$ is found to vary with time, depending on several assumptions. The plain curve indicating the largest radii corresponds to a model calculation with the standard ("hot") atmospheric boundary condition (Burrows et al. 2000b). This "hot" model thus appears to reproduce satisfactorily the measured radius.

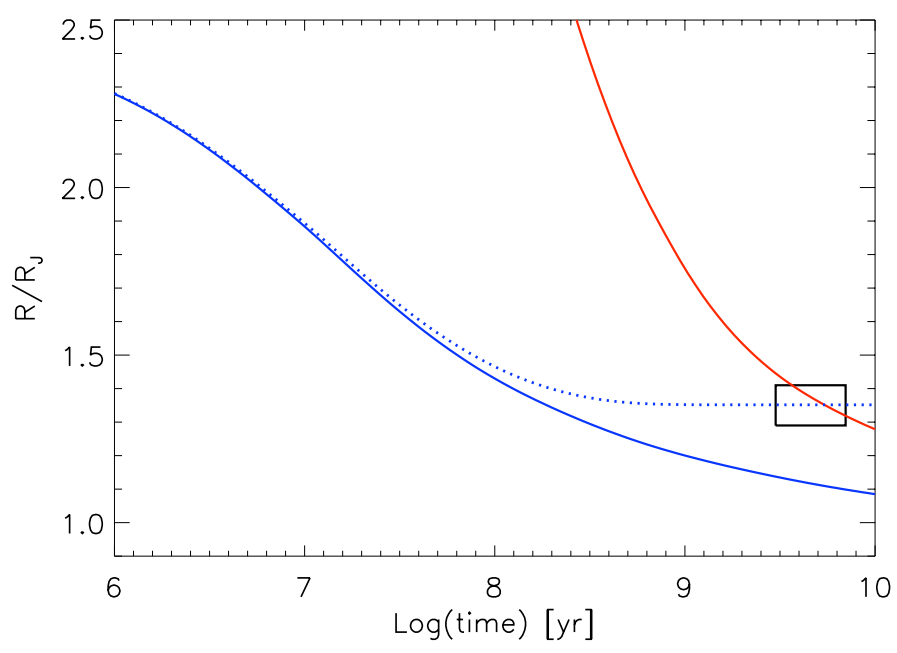

Figure 8.4: Evolution of the radius of a solar composition $0.69 \mathrm{M}_{\mathrm{J}}$ planet with $T_{\mathbf{\sigma}}=1400 \mathrm{~K}$. The plain lines correspond to standard evolution models with the "hot" (larger radii) and "cold" (smaller radii) atmospheric boundary condition. The dotted curve corresponds to an evolution calculation with dissipation, and in the "cold" case. The box indicates inferred radii and ages of HD209458b. [Adapted from Guillot \& Showman (2002)]

However, fig. 8.4 shows that a model calculated with a more realistic ("cold") atmospheric boundary condition fails to reproduce the observations ${ }^{1}$. The problem becomes even more severe if one accounts for the presence of a dense core (Bodenheimer et al. 2001). Of course, a slower

\footnotetext{
${ }^{1}$ Note that, as can be seen from fig. 8.2, the characteristic time of contraction of an irradiated planet cannot be made arbitrarily small by an increase of the initial radius. One therefore has to start the calculation from a finite initial time step, which strongly depends on the surface boundary temperature. In the hot case, this initial time step is larger than in the cold case. This is why the two evolution curves do not appear to have started from the same initial condition.
} 
evolution can be found if one assumes a larger mass, or higher interior opacities, but it appears very difficult to obtain a fair match to the observations that way.

Instead, Bodenheimer et al. (2001) and Guillot \& Showman (2002) propose that energy is dissipated by stellar tides and contributes to the energy budget in the atmosphere. In this case the equation of energy conservation in the planetary interior (eq. (2.4)) is calculated using $\dot{\epsilon}>0$. The dotted line in fig. 8.4 corresponds to the dissipation of $\dot{E} \equiv \int \dot{\epsilon} d m=1.8 \times 10^{26} \mathrm{erg} \mathrm{s}^{-1}$ (which corresponds to less than $0.1 \%$ of the globally-averaged absorbed stellar heat flux) at the planet's center. In that case, one finds that the evolution is very similar to that of stars: an equilibrium is found, in which the planet's evolution is effectively halted, as long as the star dissipates its energy into the planet.

Other cases corresponding to dissipation in external regions are also calculated by Guillot \& Showman (2002) but are not shown here. It is sufficient to mention that a dissipation of a small fraction ( $1 \%$ or less) of the stellar photons to deeper levels than by radiative transfer is sufficient to explain the measured radius.

\subsubsection{Evaporation/survival of Pegasi planets}

The radii predicted by models (Guillot et al. 1996) and the one measured for HD209458b (Brown et al. 2001) both point to a relatively modest inflation $(\sim 50 \%)$ of Pegasi planets as compared to Jupiter (see fig. 6.8). This point is crucial for the survival of these objects so close to their star.

A first important consequence is that they do not suffer from Roche lobe overflows (see fig. 8.2). Were it the case, the planets would be very rapidly lost due to the mass-radius relation implying an increase of radius for decreasing mass.

However, mass loss is expected to proceed simply through the escape of chemical species from the planets' exospheres. This happens when a particle acquires a velocity larger than the planet's escape speed. This can occur either by the escape of particles in the tail of the Maxwell-Boltzmann velocity distribution (thermal escape) or because of the production of hot ions by stellar ultraviolet radiation (non-thermal escape).

Thermal escape is difficult to estimate because the temperature of the exosphere is unknown. One possibility is that it could be limited by the ionization of $\mathrm{H}$ atoms near $10,000 \mathrm{~K}$. Even in this relatively hot case, it is found to yield a relatively small evaporation of the planet. Non-thermal escape is generally found to be more significant. Extrapolating results for Jupiter for a $10^{4}$ higher flux of photons, Guillot et al. (1996) find that a gas giant at 0.05 AU from a solar-type star with a mass of a $1 \mathrm{M}_{\mathrm{J}}$ would loose $\sim 10^{34}$ hydrogen atoms per second. Only $\sim 0.5 \%$ of its mass would be lost over the main sequence lifetime of the star. Lin et al. (1996) also reach a similar conclusion.

Very recent observations by Vidal-Madjar et al. (2003) tend to confirm these estimates. Using HST observations, these authors observe that HD209458b appears much bigger when observed in Lyman $\alpha$ than at other wavelengths. They interpret this finding by the presence of escaping hydrogen atoms, and infer a minimum escape flux of around $10^{10} \mathrm{~g} \mathrm{~s}^{-1}$. This is, coincidentally or not, almost exactly what was predicted in $1996\left(\sim 1.7 \times 10^{10} \mathrm{~g} \mathrm{~s}^{-1}\right)$.

Pegasi planets thus appear to be able to survive their proximity to their parent star, even if they are made of hydrogen and helium. In the case of planets made of denser material, the situation would be of course more comfortable. However, I stress that only rough estimates of this important problem have been made so far. Our best argument to decide that gas giant survive very close to their star is that they are observed to be there! 


\subsection{Tidal effects}

One of the specificity of Pegasi planets is that most of them have circular orbits. This indicates that tides raised by the planet on the star acted to strongly damp the planets' orbital eccentricities. The other specificity is that the tides raised by the star on the planet are expected to rapidly drive them into synchronous rotation (Guillot et al. 1996; Marcy et al. 1997; Lubow et al. 1997). This can be shown by considering the time scale to tidally despin the planet (Goldreich \& Soter 1966; Hubbard 1984):

$$
\tau_{\text {syn }} \approx Q\left(\frac{R^{3}}{G M}\right)\left(\omega-\omega_{\mathrm{s}}\right)\left(\frac{M}{M_{\star}}\right)^{2}\left(\frac{a}{R}\right)^{6},
$$

where $Q, R, M, a, \omega$ and $\omega_{\mathrm{s}}$ are the planet's tidal dissipation factor, radius, mass, orbital semimajor axis, rotational angular velocity, and synchronous (or orbital) angular velocity. $M_{*}$ is the star's mass. Factors of order unity have been omitted. A numerical estimate for HD209458b (with $\omega$ equal to the current Jovian rotation rate) yields a spindown time $\tau_{\mathrm{syn}} \sim 3 Q$ years. Any reasonable dissipation factor $Q$ (see Marcy et al. 1997; Lubow et al. 1997) shows that HD209458b should be led to synchronous rotation in less than a few million years, i.e. on a time scale much shorter than the evolution timescale. Like other Pegasi planets, HD209458b is therefore expected to be in synchronous rotation with its 3.5-day orbital period.

Nevertheless, stellar heating drives the atmosphere away from synchronous rotation, raising the possibility that the interior's rotation state is not fully synchronous. Here, I discuss (1) the energies associated with the planet's initial transient spindown, and (2) the possible equilibrium states that could exist at present.

\subsubsection{Spindown energies}

Angular momentum conservation requires that as the planet spins down, the orbit expands. The energy dissipated during the spindown process is the difference between the loss in spin kinetic energy and the gain in orbital energy:

$$
\dot{E}=-\frac{d}{d t}\left(\frac{1}{2} k^{2} M R^{2} \omega^{2}-\frac{1}{2} M a^{2} \omega_{\mathrm{s}}^{2}\right),
$$

where $k$ is the dimensionless radius of gyration $\left(k^{2}=I / M R^{2}, I\right.$ being the planet's moment of inertia). The orbital energy is the sum of the potential gravitational energy and of the kinetic energy of the planet on its orbit and is hence negative by convention. The conservation of angular momentum implies that the rate of change of $\omega_{\mathrm{s}}$ is constrained by that on $\omega$ :

$$
\frac{d}{d t}\left(M a^{2} \omega_{\mathrm{s}}+k^{2} M R^{2} \omega\right)=0 .
$$

The fact that the planetary radius changes with time may slightly affect the quantitative results. However, since $\tau_{\text {syn }}$ appears to be so short, it can be safely neglected in this first order estimate. $R$ being held constant, it is straightforward to show, using Kepler's third law, that:

$$
\dot{E}=-k^{2} M R^{2}\left(\omega-\omega_{\mathrm{s}}\right) \dot{\omega} .
$$

The total energy dissipated is $E \approx k^{2} M R^{2}\left(\omega_{\mathrm{s}}-\omega\right)^{2} / 2$, neglecting variation of the orbital distance. Using the moment of inertia and initial rotation rate of Jupiter $\left(k^{2}=0.26\right.$ and $\omega=$ 
$1.74 \times 10^{-4} \mathrm{~s}^{-1}$ ), we obtain for HD209458b $E \approx 4 \times 10^{41} \mathrm{erg}$. If this energy was dissipated evenly in the planet, this would correspond to a global increase of the temperature of the planet of $1400 \mathrm{~K}$.

By definition of the synchronization timescale, the dissipation rate can be written:

$$
\dot{E}=\frac{k^{2} M R^{2}\left(\omega-\omega_{\mathrm{s}}\right)^{2}}{\tau_{\mathrm{syn}}} .
$$

With $Q=10^{5}$, a value commonly used for Jupiter, $\tau_{\text {syn }} \sim 3 \times 10^{5}$ years and the dissipation rate is then $\sim 10^{29} \mathrm{erg} \mathrm{s}^{-1}$, or 35,000 times Jupiter's intrinsic luminosity. Lubow et al. (1997) have suggested that dissipation in the radiative zone could yield lower values of $Q$ before spindown has occurred; if so, the initial energy deposition rate could be as large as $10^{-2} \mathrm{~L}_{\odot}=2 \times 10^{31} \mathrm{erg} \mathrm{s}^{-1}$, but this would last for only $\sim 100$ years.

The thermal pulse associated with the initial spindown is large enough that, if the energy is dissipated in the planet's interior, it may affect the planet's radius. It has previously been argued (Burrows et al. 2000b) that Pegasi planets must have migrated inward during their first $10^{7}$ years of evolution; otherwise, they would have contracted too much to explain the observed radius of HD209458b. But the thermal pulse associated with spindown was not included in the calculation, and this extra energy source may allow later migration to be consistent with HD209458b's radius. Unfortunately, therefore, it may be difficult to derive constraints on the migration time scale from radii measurements.

On the other hand, it seems difficult to invoke tidal synchronization as the missing heat source necessary to explain HD209458b's present radius. High dissipation rates are possible if $\tau_{\text {syn }}$ is small, but $\dot{E}$ would drop as soon as $t>\tau_{\text {syn }}$ in the absence of a mechanism to prevent synchronization. The most efficient way of slowing the contraction of Pegasi planets would then be to invoke $\tau_{\text {syn }} \sim$ $10^{10}$ years. In that case, the energy dissipated becomes $\dot{E} \sim 10^{24} \mathrm{erg} \mathrm{s}^{-1}$, i.e. at least two orders of magnitude smaller than that necessary to significantly affect the planet's evolution. Dissipation of the energy due to transient loss of the planet's initial spin energy is therefore unlikely to be an substantial-enough source of present-day heating to explain the radius of HD209458b.

Another possible source of energy is through circularization of the orbit. Bodenheimer et al. (2001) show that the resulting energy dissipation could reach $10^{26} \mathrm{erg} \mathrm{s}^{-1}$ if the planet's tidal $Q$ is $10^{6}$ and if a hypothetical companion planet pumps HD209458b's eccentricity to values near its current observational upper limit of 0.04. If such a companion is absent, however, the orbital circularization time is $\sim 10^{8}$ years, so this source of heating would be negligible at present. Longer circularization times of $10^{9}-10^{10}$ years would allow the heating to occur until the present-day, but its magnitude is then reduced to $10^{25} \mathrm{erg} \mathrm{s}^{-1}$ or lower, which is an order of magnitude smaller than the dissipation required.

\subsubsection{The equilibrium state}

The existence of atmospheric winds implies that the atmosphere is not synchronously rotating. Because dynamics can transport angular momentum vertically and horizontally (including the possibility of downward transport into the interior), the interior may evolve to an equilibrium rotation state that is asynchronous.

Let us split the planet into an "atmosphere", a part of small mass for which thermal effects are significant, and an "interior" encompassing most of the mass which has minimal horizontal thermal contrasts. Suppose (since $\tau_{\text {syn }}$ is short) that the system has reached steady state. The two possible cases are illustrated by fig. 8.5 and depend on the physical mechanisms that determine the 
gravitational torque caused on the atmosphere (see Showman \& Guillot 2002 for a more detailed discussion).

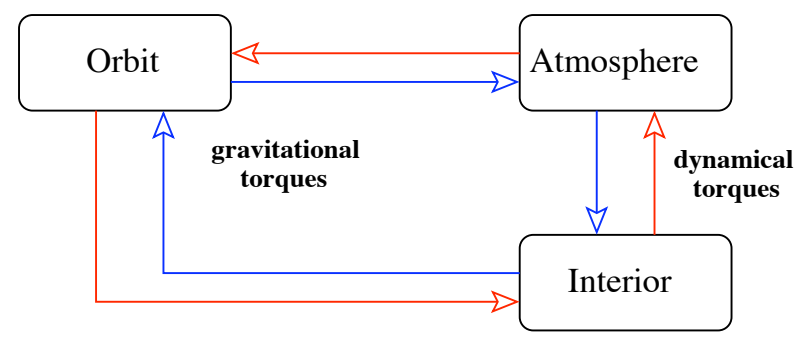

Figure 8.5: Angular momentum flow between orbit, interior, and atmosphere for a Pegasi planet in steady state. Arrows indicate flow of prograde angular momentum (i.e., that with the same sign as the orbital angular momentum) for two cases: Anticlockwise: Gravitational torque on atmosphere is retrograde (i.e., adds westward angular momentum to atmosphere). For torque balance, the gravitational torque on the interior must be prograde (i.e., eastward). These gravitational torques must be balanced by fluid-dynamical torques that transport retrograde angular momentum from atmosphere to interior. Clockwise: Gravitational torque on atmosphere is prograde, implying a retrograde torque on the interior and downward transport of prograde angular momentum from atmosphere to interior. Atmosphere will superrotate if gravitational torques push atmosphere away from synchronous (as on Venus). It will subrotate if gravitational torques synchronize the atmosphere (e.g., gravity-wave resonance).

A simple estimate illustrates the extent of nonsynchronous rotation possible in the interior. Suppose that the globally-averaged flux of absorbed starlight is $F_{\mathbf{a}}$, which is of order $10^{8} \mathrm{erg} \mathrm{s}^{-1} \mathrm{~cm}^{-2}$ for Pegasi planets near $0.05 \mathrm{AU}$, and that the globally-averaged flux of kinetic energy transported from the atmosphere to the interior is $\eta F_{\mathbf{a}}$, where $\eta$ is small and dimensionless. If this kinetic energy flux is balanced by dissipation in the interior with a spindown timescale of $\tau_{\mathrm{syn}}$, then the deviation of the rotation frequency from synchronous is

$$
\omega-\omega_{s}=\left(\frac{4 \pi \eta F_{\mathbf{\sigma}} \tau_{\mathrm{syn}}}{k^{2} M}\right)^{1 / 2}
$$

Experience with planets in our solar system suggests that atmospheric kinetic energy is generated at a flux of $10^{-2} F_{\text {a }}$, and if all of this energy enters the interior, then $\eta \sim 10^{-2}$. Using a spindown time of $3 \times 10^{5}$ years then implies $\omega-\omega_{s} \sim 2 \times 10^{-5} \mathrm{~s}^{-1}$, which is comparable to the synchronous rotation frequency. The implied winds in the interior are then of order $\sim 2000 \mathrm{~m} \mathrm{~s}^{-1}$. Even if $\eta$ is only $10^{-4}$, the interior's winds would be $200 \mathrm{~m} \mathrm{~s}^{-1}$. The implication is that the interior's spin could be asynchronous by up to a factor of two, depending on the efficiency of energy and momentum transport into the interior.

Any scenario involving different rotation rates of the atmosphere and interior inevitably leads to significant energy dissipation. Since we have considered situations for which the system is in gravitational equilibrium, the energy associated with the flow is provided by the stellar photons. A fraction of the absorbed stellar flux is therefore dissipated at levels other than what would be predicted from radiative transfer. Depending on whether the energy is dissipated at low pressures or deep in the interior, the consequences for the planet's evolution are very different. If energy is dissipated in the high atmosphere, as may be the case for gravity waves, the effect on the evolution 
will be small. If it is dissipated in the interior, as in the case of a Kelvin-Helmoltz instability, this could potentially be the dominant process governing the planet's evolution.

\subsection{Atmospheric dynamics}

I have so far implicitly assumed that Pegasi planets have uniform atmospheres. Because of the strong inhomogeneous stellar irradiation, and the near-synchronous rotation, this hypothesis is in fact probably very far from reality. The consequences of the presence of day/night temperature variations for the evolutions are found to affect only weakly the planet's contraction (Guillot \& Showman 2002). Here, I analyse the consequences for the atmosphere of Pegasi planets, on the basis of the articles by Guillot (2001) and Showman \& Guillot (2002).

\subsubsection{Timescales}

Temperature variations across planetary atmospheres are governed by the time required for the atmosphere to absorb the stellar heat, to radiate its heat to space, and by the characteristic advective time scales.

The radiative heating/cooling timescale can be estimated by a ratio between the thermal energy within a given layer and the layer's net radiated flux. In the absence of dynamics, absorbed solar fluxes balance the radiated flux, but dynamics perturbs the temperature profile away from radiative equilibrium. Suppose the radiative equilibrium temperature at a particular location is $T_{\text {rad }}$ and the actual temperature is $T_{\mathrm{rad}}+\Delta T$. The net flux radiated toward outer space is then $4 \sigma T_{\mathrm{rad}}^{3} \Delta T$ and the radiative timescale is

$$
\tau_{\mathrm{rad}} \sim \frac{P}{g} \frac{c_{p}}{4 \sigma T^{3}} .
$$

This timescale is thus particularly dependent on the characteristic temperature of the atmosphere. For our giant planets, $T \sim 200 \mathrm{~K}$, so that the radiative timescale is long, i.e. about a year at 1 bar. This is to be compared, e.g. to the rotation period, which is of the order of 10 hours for Jupiter and Saturn. Their atmospheres are thus found to be relatively uniform. However, as shown in fig. 8.6, Pegasi planets have ten times hotter atmospheres, so that $\tau_{\text {rad }}$ is of the order of 1 day at photospheric levels, to be compared with their rotation period of $\sim 4$ days.

The timescale for advection by winds is more difficult to estimate. Guillot (2001) and Showman \& Guillot (2002) use a shear instability criterion: assuming that the convective core is locked into synchronous rotation, they assume that at upper levels winds build up with increasing altitude only if they do not exceed the Kelvin-Helmholtz instability criterion (Chandrasekhar 1961), i.e. if:

$$
R i=\frac{N^{2}}{(d u / d z)^{2}}>\frac{1}{4},
$$

where $R i$ is the Richardson number, and $N^{2}=\left(g / H_{P}\right)\left(\nabla_{\mathrm{ad}}-\nabla_{T}\right)$ ( $N$ is the Brünt-Vaisala frequency). This thus implies a constraint on the wind shear $d u / d z$. The resulting timescale is shown as dashed curves in fig. 8.6, for both the "cold" and "hot" cases.

At pressures exceeding 0.1 bar, radiation is slower than the maximal advection by zonal winds, but by less than one order of magnitude. The consequent day/night temperature difference $\Delta T_{\text {day-night }}$ to be expected is:

$$
\frac{\Delta T_{\text {day }- \text { night }}}{\Delta T_{\text {rad }}} \sim 1-e^{-\tau_{\text {zonal }} / \tau_{\text {rad }}} .
$$




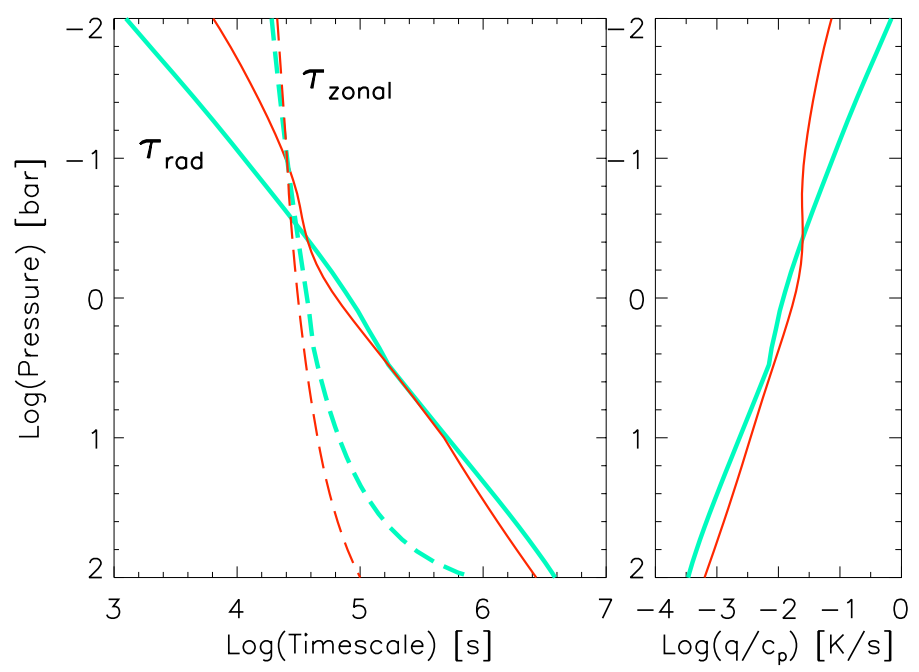

Figure 8.6: Left: Characteristic time scales as a function of pressure level. $\tau_{\text {zonal }}$ is the minimal horizontal advection time (dashed). $\tau_{\text {rad }}$ is the timescale necessary to cool/heat a layer of pressure $P$ and temperature $T$ by radiation alone (solid). For each case, the thin black and thick gray lines correspond to the "hot" and "cold" models. Right: Approximate cooling/heating rate as a function of pressure. [From Showman \& Guillot (2002)]

where $\Delta T_{\text {rad }}$ is the day-night difference in radiative equilibrium temperatures. Rough estimates from Fig. 8.6 suggest that $\tau_{\text {zonal }} / \tau_{\text {rad }} \sim 0.3$ at 1 bar, implying that $\Delta T_{\text {day }- \text { night }} / \Delta T_{\text {rad }} \sim 0.3$. If $\Delta T_{\text {rad }}=1000 \mathrm{~K}$, this would imply day-night temperature differences of $300 \mathrm{~K}$ at 1 bar. Values of $\Delta T_{\text {day-night }}$ even closer to $\Delta T_{\text {rad }}$ are likely given the fact that slower winds will lead to an even more effective cooling on the night side and heating on the day side.

The small radiative time scale implies that, for the day-night temperature difference to be negligible near the planet's photosphere, atmospheric winds would have to be larger than the maximum winds for the onset of shear instabilities.

\subsubsection{Possible circulation and atmospheric chemistry}

As discussed by Showman \& Guillot (2002), the intense stellar radiation is expected to drive both zonal and meridional winds, but the atmospheric circulation is unknown. However, they note that even if locked into synchronous rotation, the atmospheres of Pegasi planets are characterized by relatively low $(\sim 0.1)$ Rossby numbers. This implies that the Coriolis force plays a very important role and that zonal circulation is favored over meridional circulation.

A preliminary numerical simulation with a global circulation model by Showman \& Guillot (2002) indicates that a fast superrotating equatorial jet develops, and that the atmosphere is globally superrotating, a situation very similar to that of Venus. This situation is depicted in fig. 8.7. It is interesting to notice that this kind of circulation pattern implies that the equator to pole temperature variation is even more pronounced than the day to night one.

However, the consequences for cloud formation and chemistry in the atmospheres of Pegasi planets are still unclear. The solution to that problem depends in fact on whether the heat- 


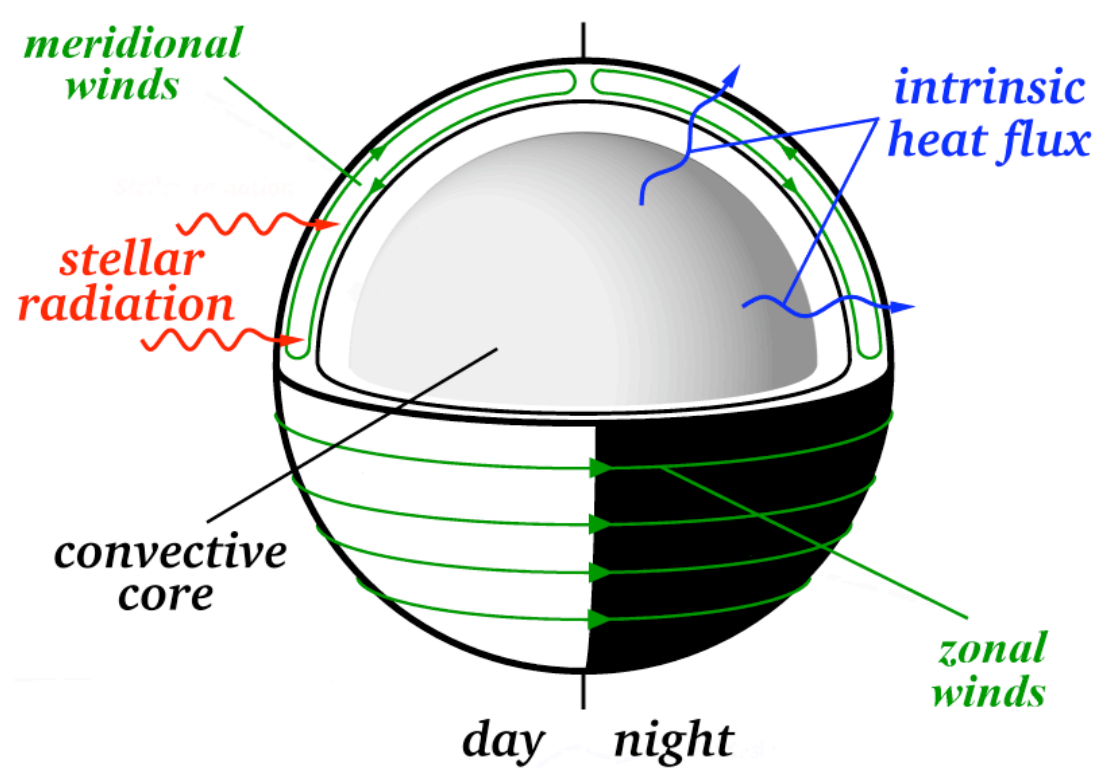

Figure 8.7: Conjectured dynamical structure of Pegasi planets: At pressures larger than 100800 bar, the intrinsic heat flux must be transported by convection. The convective core is at or near synchronous rotation with the star and has small latitudinal and longitudinal temperature variations. At lower pressures a radiative envelope is present. The top part of the atmosphere is penetrated by the stellar light on the day side. The spatial variation in insolation should drive winds that transport heat from the day side to the night side. [From Guillot (2001); Showman \& Guillot (2002)].

ing/cooling is mostly balanced by vertical motions (in which case clouds would tend to form at the substellar point, on the day side), or by horizontal advection. The latter seems to be favored by the simulations and it is instructive to discuss it further.

Let us assume that a superrotating wind advects air roughly on constant pressure levels (negligible vertical advection). In that case, air is cooled on the night side, then it is intensely heated on the day side. As a consequence, any chemical species that condenses on the night side and forms clouds there will evaporate on the day side. The night side should then be relatively cloudy, while the day side would be clear (low albedo). But this circulation has another very important consequence for atmospheric chemistry: most abundant species that condense on the night side are, according to the estimates from Rossow (1978), expected to settle down on short timescales (Guillot 2001). Because, according to our hypothesis, the air is transported on isobars, when it reaches the day side, the condensing species are undersaturated everywhere down to the condensation level on the night side.

The magnitude of this effect can be estimated as follows: Let us assume that on the day side, the saturation abundance of the condensing species, $x=p / P$ is maximal and equal to $x^{\star}$ at $P=P_{\text {day }}^{\star}$ (the condensation level on the day side). On the night side, the temperature is lower. Equation (7.4) and (7.12) can be used to show that the abundance at saturation on the night side 
becomes:

$$
\ln x\left(P_{\text {day }}^{\star}\right)=\ln x^{\star}-\beta \ln \left(T_{\text {day }} / T_{\text {night }}\right) .
$$

In order to reach condensation, i.e. $x=x^{\star}$, one has to penetrate deeper into the atmosphere. Eq. (7.13) implies that on the night side,

$$
\ln x(P)=\ln x\left(P_{\text {day }}^{\star}\right)+\left(\beta \nabla_{T}-1\right) \ln \left(P / P_{\text {day }}^{\star}\right),
$$

assuming that $\nabla_{T}$ is constant. Using eq. (8.14), one obtains the condensation pressure on the night side:

$$
\frac{P_{\text {night }}^{\star}}{P_{\text {day }}^{\star}}=\left(\frac{T_{\text {day }}}{T_{\text {night }}}\right)^{\beta /\left(\beta \nabla_{T}-1\right)} .
$$

Using $\beta \sim 10, \nabla_{T} \sim 0.15$ and $T_{\text {day }} / T_{\text {night }} \sim 1.2$, one finds $P_{\text {night }}^{\star} \sim 38 P_{\text {day }}^{\star}$, a very significant variation of the condensation pressure. This implies that air flowing on constant pressure levels around the planet would lead to a rapid depletion of any condensing species on the day side, compared to what would be predicted from chemical equilibrium calculations. This can potentially also remove important absorbing gases from the day side, as in the case of $\mathrm{TiO}$, which can be removed by $\mathrm{CaTiO}_{3}$ condensation, or Na, removed by $\mathrm{Na}_{2} \mathrm{~S}$ condensation (Lodders 1999). Of course, most of the variation depends on the exponential factor $\beta /\left(\beta \nabla_{T}-1\right)$, which is infinite in the limit when the atmospheric temperature profile and the condensation profile are parallel to each other. In the discussion, we implicitly assumed $\beta \nabla_{T}-1>0$; however, when the atmosphere is close to an isotherm, this factor can become negative. In this case the day/night effect is even more severe, as the condensing species is entirely removed from this quasi-isothermal region.

The rapid circulation from the night side to the day side can also lead to a disequilibrium chemistry for non-condensing species when the reaction timescales are longer than the advection timescale $\left(\sim\right.$ a day). This is for example the case of the $\mathrm{N}_{2}$ to $\mathrm{NH}_{3}$ reaction in Gl229B (Saumon et al. 2000b), but many other chemical species should be affected.

\subsubsection{Observational consequences}

The structure and evolution of Pegasi planets is much more complex than envisioned when these planets were first discovered. The possibility of dissipation by stellar tides is interesting because it will be directly tested by observations and because this phenomenon is poorly understood even for the planets of our solar system. However, the drawback is that it should be more difficult to infer the planets' global compositions from radii measurements, as first suggested (Guillot 1999b).

However, our understanding of these objects should be greatly increased by the numerous direct or indirect observations that are now possible. With several ground programs (STARE, VULCAN), accepted space missions (COROT, MONS, MOST) and proposed ones (KEPLER, EDDINGTON) aiming at detecting photometric transits of Pegasi planets, there is indeed a good chance that statistically significant information on e.g. the mass radius relationship of Pegasi planets can be gathered.

Measurement of starlight reflected from these planets may allow the albedo to be estimated. Because the star-planet-Earth angle changes throughout the planet's orbit, crude information on the scattering properties of the atmosphere (e.g., isotropic versus forward scattering) may be obtainable. Asymmetries in the reflected flux as the planet approaches and recedes from the transit could give information on the differences of albedo near the leading and trailing terminators, which would help constrain the dynamics. Finally, transit observations of Pegasi planets using high resolution spectroscopy should in the near future yield constraints on the atmospheric temperature, 
cloud/haze abundance, and winds (Seager \& Sasselov 2000; Brown 2001; Hubbard et al. 2001). If these measurements are possible during the ingress and egress, i.e., the phases during which the planets enters and leaves the stellar limb, respectively, asymmetries of the planetary signal should be expected and would indicate zonal heat advection at the terminator.

\section{Remerciements}

Merci à toute l'organisation de l'école, et à tout ceux qui m'ont fourni du matériel pour ce cours, ecrit difficilement, en pensant à toi petite sœur. 


\section{References}

Ackerman A.S. \& Marley M.S., ApJ 556, 872 (2001).

Acuña M.H., Connerney J.E.P. et Ness N.F., J. Geophys. Res. 88, 8771 (1983).

Alexander D.R. \& Ferguson J.W., ApJ 437, 879 (1994).

Allard F. \& Hauschildt P.H., ApJ 445, 433 (1995).

Allard F., Hauschildt P.H., Baraffe I. \& Chabrier G., ApJL 465, L123 (1996).

Allard F., Hauschildt P.H., Alexander D.R., Tamanai A., \& Schweitzer A., ApJ 556, 357 (2001).

Anderson J.D., Campbell J.K., Jacobson R.A., et al., J. Geophys. Res. 92, 14877 (1987).

Bahcall J.N., Pinsonneault M.H., Rev. Mod. Phys. 67, 781 (1995).

Bailer-Jones, C.A.L. \& Mundt R., A $\& A$ 374, 1071 (2001).

Basri G., Ann. Rev. Aston. Astrophys. 38, 485 (2000).

Barman T., Hauschildt P.H., Allard F., ApJ 556, 885 (2001).

Beule D., Ebeling W., Förster A., Juranek H., Nagel S., Redmer R. \& Röpke G., Phys. Rev. B 59, 4177 (1999).

Birnbaum G., J. Quant. Spectrosc. Radiat. Transfer 21, 597 (1979).

Bodenheimer P., Hubickyj O. \& Lissauer J.J., Icarus 143, 2 (2000).

Bodenheimer P., Lin D.N.C., Mardling R., ApJ 548, 466 (2001).

Borysow A., Borysow J., \& Fu Y., Icarus 145, 601 (2000).

Borysow A., Icarus 96, 169 (1992).

Boss A.P., ApJ 503, 923 (1998).

Boss A.P., ApJL 536, L101 (2000).

Brown T.M., ApJ 553, 1006 (2001).

Brown T.M., Charbonneau D., Gilliland R.L., Noyes R.W., Burrows A., ApJ 552, 699 (2001).

Burgasser A.J. et al., ApJL 522, L65 (1999).

Burrows A. \& Liebert J., Rev. Mod. Phys. 65, 301 (1993).

Burrows A., Marley M., Hubbard W.B., Lunine J.I., Guillot T., Saumon D., Freedman R., Sudarsky D., Sharp C., ApJ 491, 856 (1997).

Burrows A., Marley M.S. \& Sharp, C.M., ApJ 531, 438 (2000a).

Burrows A., Guillot T., Hubbard W.B., Marley M.S., Saumon D., Lunine J.I. \& Sudarsky D., ApJL 534, L97 (2000b).

Busse F.H., Ann. Rev. Fluid. Mech 10, 435 (1978).

Campbell J.K. \& Synnott S.P., Astron. J. 90, 364 (1985).

Campbell J.K. \& Anderson J.D., Astron. J. 97, 1485 (1989).

Carlson B.E., Lacis A.A., Rossow W.B., ApJ 388, 648 (1992).

Carlson R. et al., Science 274, 385 (1996).

Cavazzoni C., Chiarotti G.L., Scandolo S., Tosatti E., Bernasconi M., \& Parrinello M., Science 283, 44 (1999).

Celliers et al., Phys. Rev. Let. 84, 5564 (2000).

Chabrier G., Saumon D., Hubbard W.B., Lunine J.I., ApJ 391, 817 (1992).

Chabrier G. \& Baraffe I., A\& A 327, 1039 (1997).

Chabrier G. \& Baraffe I., Ann. Rev. Aston. Astrophys. 38, 337 (2000).

Chabrier G., Baraffe I., Allard F. \& Hauschildt P., ApJL 542, L119 (2000a).

Chabrier G., Baraffe I., Allard F. \& Hauschildt P., ApJ 542, 464 (2000b).

Chandrasekhar S., "Stellar Structure and Evolution", The University of Chicago press, Chicago (1939).

Chandrasekhar S., "Hydrodynamic and hydromagnetic stability", Dover, New York (1961). 
Charbonneau D., Brown T.M., Latham D.W., Mayor, M., ApJ 529, L45 (2000).

Clayton D.D., "Principles of stellar evolution and nucleosynthesis", McGraw-Hill Book Company, New-York (1968).

Cohen E.R., Taylor B.N., Rev. Mod. Phys. 59, 1121 (1986).

Collins G.W. et al., Science 281, 1179 (1998).

Connerney J.P., Acuña M.H. \& Ness N.F., J. Geophys. Res. 86, 3623 (1982).

Conrath B.J., Gautier D., Hanel R., Lindal G., Marten A., ApJ 282, 807 (1984).

Conrath B.J., Hanel R.A. \& Samuelson R.E. In Origin and Evolution of Planetary and Satellite Atmospheres (eds. S.K. Atreya, J.B. Pollack, and M.S. Matthews), Univ. of Arizona Press, Tucson, pp. 513-538 (1989)

Conrath B.J. \& Gautier D., Icarus 144, 124 (2000).

Cotton W.R. \& Anthes R.A., "Storm and cloud dynamics", Academic Press, Inc., San Diego (1989).

Da Silva L.B. et al., Phys. Rev. Let. 78, 483 (1997).

Davies M.E., Abalakin V.K., Lieske J.H., Seidelmann P.K., Sinclair A.T., Sinzi A.M., Smith B.A. \& Tjuflin Y.S., Celestial Mech. 29, 309 (1986).

Emanuel K.A., "Atmospheric Convection", Oxford Univ. Press, New-York (1994).

Fegley B.J. \& Lodders K., Icarus 110, 117 (1994).

Fegley B.J. \& Lodders K., ApJL 472, L37 (1996).

Folkner W.M., Woo R., Nandi S., J. Geophys. Res. 103, 22831 (1998).

Fontaine G., Graboske H.C. \& van Horn H.M., ApJS 35, 293 (1977).

Fowler W.A., Caughlan G.R. \& Zimmerman B.A., Ann. Rev. Aston. Astrophys. 13, 69 (1975).

Galli G. et al., Phys. Rev. B 61, 909 (2000).

Gautier D., Owen, T. In Origin and Evolution of Planetary and Satellite Atmospheres (eds. S.K. Atreya, J.B. Pollack, and M.S. Matthews), University of Arizona Press, Tucson, pp. 487-512 (1989)

Gautier D., Hersant F., Mousis O. \& Lunine J.I., ApJL 559, L183 (2001).

Geballe T.R., Kulkarni S.R., Woodward C.E. \& Sloan G.C., ApJL 467, L101 (1996).

Gierasch P.J. \& Conrath B.J., J. Geophys. Res. 98, 5459 (1993).

Gladman B. et al., Nature 412, 163 (2001).

Goldreich P. \& Soter S., Icarus 5, 375 (1966).

Goody R.M. \& Yung Y.L., "Atmospheric radiation", Oxford University Press, New York (1989).

Goukenleuque C., Bézard B., Joguet B., Lellouch E., Freedman R., Icarus 143, 308 (2000).

Graboske H.C., Pollack J.B., Grossman A.S. \& Olness R.J., ApJ 199, 265 (1975).

Griffith C.A., Yelle R.V. \& Marley M.S., Science 282, 2063 (1998).

Griffith C.A. \& Yelle R.V., ApJL 519, L85 (1999).

Gudkova T.V. \& Zharkov V.N., Plan. Space. Sci. 47, 1201 (1999).

Guillot T., Gautier D., Chabrier G., Mosser B., Icarus 112, 337 (1994a).

Guillot T., Chabrier G., Morel P. \& Gautier D., Icarus 112, 354 (1994b).

Guillot T. \& Morel P., A\&AS 109, 109 (1995).

Guillot T., Science 269, 1697 (1995).

Guillot T., Burrows A., Hubbard W.B., Lunine J.I. \& Saumon D., ApJ 459, L35 (1996).

Guillot T., Gautier D. \& Hubbard W.B., Icarus 130, 534 (1997).

Guillot T., Plan. Space. Sci. 47, 1183 (1999a).

Guillot T., Science 286, 72 (1999b).

Guillot T. Atmospheric circulation of hot Jupiters. In Planetary Systems in the Universe: Observation, Formation and Evolution, ASP Conf. series, A. Penny et al. eds., in press (2001) 
Guillot T. \& Showman A., A\&SA 385, 156 (2002).

Hayashi C., Prog. Theo. Phys. Suppl. 70, 35 (1981).

Henry G.W., Marcy G.W., Butler R.P., Vogt, S.S., ApJ 529, L41-L44 (2000).

Hillenbrand L.A. \& Carpenter J.M., ApJ 540, 236 (2000).

Holmes N.C., Ross M. \& Nellis W.J., Phys. Rev. B 52, 15835 (1995).

Hubbard W.B., ApJ 152, 745 (1968).

Hubbard W.B. \& Lampe M., ApJS 18, 297 (1969).

Hubbard W.B., Icarus 30, 305 (1977).

Hubbard W.B., Icarus 52, 509 (1982).

Hubbard W.B., "Planetary Interiors", Van Nostrand Reinhold Co., Inc., New York (1984).

Hubbard, W.B. In Origin and Evolution of Planetary and Satellite Atmospheres, S. K. Atreya, J. B. Pollack, and M. S. Matthews, eds., University of Arizona Press, Tucson, pp. 539-563 (1989)

Hubbard W.B. \& Marley M.S., Icarus 78, 102 (1989).

Hubbard W.B., Pearl J.C., Podolak M. \& Stevenson D.J., in Neptune and Triton, ed. D. P. Cruikshank (Tucson: Univ. of Arizona Press), pp. 109-138 (1995)

Hubbard W.B. et al., Physics of Plasmas 4, 2011 (1997).

Hubbard W.B., Guillot T., Marley M.S., Burrows A., Lunine J.I. \& Saumon D.S., Plan. Space. Sci. 47, 1175 (1999).

Hubbard W.B., Icarus 137, 196 (1999).

Hubbard W.B., Fortney J.J., Lunine J.I., Burrows A., Sudarsky D. \& Pinto P., ApJ 560, 413 (2001).

Ingersoll A.P., Kanamori H. \& Dowling T.E., Geophys. Res. Lett. 21, 1083 (1994).

Ingersoll A.P., Barnet C.D., Beebe R.F. et al., in Neptune and Triton, ed. D.P. Cruikshank, University of Arizona Press, Tucson, 613 (1995)

Jeffreys H., M.N.R.A.S. 83, 350 (1923).

Kerley G.I., Phys. Earth Planet. Interiors 6, 78 (1972).

Kippenhahn R. \& Weigert A., "Stellar structure and evolution", Springer-Verlag, Berlin (1991).

Kirkpatrick J.D. et al., ApJ 519, 802 (1999).

Klepeis J.E., Schafer K.J., Barbee T.W.III, Ross M., Science 254, 986 (1991).

Knudson M.D., Hanson D.L., Bailey J.E., Hall C.A., Asay J.R. \& Anderson W.W., Phys. Rev. Let. 87, 5501 (2001).

Kunde V., Hanel R., Maguire W., Gautier D., Baluteau J.P., Marten A., Chedin A., Husson N. et Scott N., ApJ 263, 443 (1982).

Landau L., Lifschitz E., "Physique Statistique", Editions Mir, Moscow (1976).

Lenosky T.J., Bickham S.R., Kress J.D. \& Collins L.A., Phys. Rev. B 61, 1 (2000).

Lenzuni P., Chernoff D.F. \& Salpeter E.E., ApJ Suppl. 76, 759 (1991).

Liebert J., Reid I.N., Burrows A., Burgasser A.J., Kirkpatrick J.D. \& Gizis J.E., ApJL 533, L155 (2000).

Lin D.N.C., Bodenheimer P., \& Richardson D.C., Nature 380, 606 (1996).

Lindal G.F., Wood G.E., Levy G.S., Anderson J.D., Sweetnam D.N., Hotz H.B., Buckles B.J., Holmes D.P., Doms P.E., Eshleman V.R., Tyler G.L., \& Croft T.A., J. Geophys. Res. 86, 8721 (1981).

Lindal G.F., Sweetnam D.N., Eshleman V.R., J. Geophys. Res. 92, 14987 (1985).

Lindal G.F., Astron. J. 103, 967 (1992).

Lodders K., ApJ 519, 793 (1999).

Lubow S.H., Tout C.A. \& Livio M., ApJ 484, 866 (1997). 
Luhman K.L., Rieke G.H., Young E.T., Cotera A.S., Chen H., Rieke M.J., Schneider G. \& Thompson R.I., ApJ 540, 1016 (2000).

Lunine J.I., Hubbard W.B., Burrows A., Wang Y.-P. \& Garlow K., ApJ 338, 314 (1989).

Marcy G.W., Butler R.P., Williams E., Bildsten L., Graham J.R., Ghez A.M., Jernigan J.G., ApJ 481, 926 (1997).

Marcy G.W., Cochran W.D. \& Mayor M. In Protostars and Planets IV (Tucson: University of Arizona Press; eds Mannings V., Boss A.P., Russell S.S.), p. 1285 (2000)

Marley M.S., Gomez P. \& Podolak P., J. Geophys. Res. 100, 23349 (1995).

Marley M.S., Saumon D., Guillot T., Freedman R.S., Hubbard W.B., Burrows A. \& Lunine J.I., Science 272, 1919 (1996).

Marley M.S. \& McKay C.P., Icarus 138, 268 (1999).

Marley M.S., Gelino C., Stephens D., Lunine J.I. \& Freedman R., ApJ 513, 879 (1999).

Martín E.L., Basri G., Delfosse X. \& Forveille T., A $\& A$ 327, L29 (1997).

Martín E.L., Delfosse X., Basri G., Goldman B., Forveille T. \& Zapatero Osorio M.R., AJ 118, $2466(1999)$

Martín E.L., Zapatero Osorio M. R., Barrado y Navascués D., Béjar V.J.S. \& Rebolo R., ApJL 558, L117 (2001).

Mayor M. \& Queloz D., Nature 378, 355 (1995).

Mayor M., Naef D., Pepe F., Queloz D., Santos N., Udry S., Burnet M. In Planetary Systems in the Universe: Observation, Formation and Evolution, IAU Symp. 202, Eds. A. Penny, P. Artymowicz, A.-M. Lagrange and S. Russel ASP Conf. Ser, in press (2001)

Militzer B. \& Ceperley D.M., Phys. Rev. Lett. 85, 1890 (2000).

Militzer B. \& Ceperley D.M., Phys. Rev. E 63, 6404 (2001).

Mostovych A.N., Chan Y., Lehecha T., Schmitt A. \& Sethian J.D., Phys. Rev. Let. 85, 3870 (2000).

Montalbán J., D’Antona F., \& Mazzitelli I., A\&A 360, 935 (2000).

Mosser B., Maillard J.P. \& Mékarnia D., Icarus 144, 104 (2000).

Najita J.R., Tiede G.P. \& Carr J.S., ApJ 541, 977 (2000).

Nakajima T., Oppenheimer B.R., Kulkarni S.R., Golimowski D.A., Matthews K. \& Durrance S. T., Nature 378, 463 (1995).

Nefedov A.P., Sinel'shchikov V.A. \& Usachev A.D., Physica Scripta 59, 432 (1999).

Nellis W.J., Weir S.T., Mitchell A.C., Phys. Rev. B 59, 3434 (1999).

Nellis W.J., Mitchell A.C., van Thiel M., Devine G.J., Trainor R.J., Brown N., Journal Chemical Physics 79, 1480 (1983).

Ness N.F. et al., Science 233, 85 (1986).

Ness N.F. et al., Science 246, 1473 (1989).

Niemann H.B., Atreya S.K., Carignan G.R., Donahue T.M., Haberman J.A., Harpold D.N., Hartle R.E., Hunten D.M., Kaspzrak W.T., Mahaffy P.R., Owen T.C. \& Way S.H., J. Geophys. Res. 103, 22831 (1998)

Oppenheimer B.R., Kulkarni S.R., Matthews K. \& Nakajima T., Science 270, 1478 (1995).

Oppenheimer B.R., Kulkarni S.R., Matthews K. \& van Kerkwijk M.H., ApJ 502, 932 (1998).

Ouyed R., Fundamenski W.R., Cripps G.R., \& Sutherland P.G., ApJ 501, 367 (1998).

Owen T., Mahaffy P., Niemann H.B., Atreya S., Donahue T., Bar-Nun A. \& de Pater I., Nature 402, 269 (1999).

de Pater I., Massie, S.T., Icarus 62, 143 (1985).

Pearl J.C. \& Conrath B.J., J. Geophys. Res. Suppl. 96, 18921 (1991).

Pedlosky J., "Geophysical fluid dynamics", Springer-Verlag, New-York (1979). 
Pfaffenzeller O., Hohl D. \& Ballone P., Phys. Rev. Lett. 74, 2599 (1995).

Podolak M., Hubbard W.B. \& Stevenson D.J., in Uranus, eds. J. T. Bergstralh, E. D. Miner, M. S. Matthews (Tucson: Univ. of Arizona Press), pp. 29-61. (1991)

Podolak M., Weizman A. \& Marley M.S., Plan. Space. Sci. 43, 1517 (1995).

Podolak M., Podolak J.I. \& Marley M.S., Plan. Space. Sci. 48, 143 (2000).

Pollack J.B., McKay C.P. \& Christofferson B.M., Icarus 64, 471 (1985).

Pollack J.B., Hollenbach D., Beckwith S., Simonelli D.P., Roush T. \& Fong W., ApJ 421, 615 (1994).

Pollack J.B., Hubickyj O., Bodenheimer P., Lissauer J.J., Podolak M. \& Greenzweig Y., Icarus 124, $62(1996)$.

Potekhin A.Y., Baiko D.A., Haensel P. \& Yakovlev D.G., A $\& A$ 346, 345 (1999).

Prandtl L., Z. Angew. Math. Mech. 5, 136 (1925).

Rogers F.J., Physics of Plasmas 7, 51 (2000).

Ross M., Phys. Rev. B 58, 669 (1998).

Ross M. \& Yang L.H., Phys. Rev. B 64, 134210 (2001).

Rossow W.B., Icarus 36, 1 (1978).

Roulston, M.S., Stevenson, D.J. Prediction of neon depletion in Jupiter's atmosphere EOS 76, 343 (1995) [abstract].

Salpeter E.E., ApJ 181, L83 (1973).

Sanchez-Lavega A., Colas F., Lecacheux J., Laques P., Parker D., \& Miyazaki I., Nature 353, 397 (1991).

Saumon D., Hubbard W.B., Chabrier G. \& Van Horn H.M., ApJ 391, 827 (1992).

Saumon D., Chabrier G. \& Van Horn H.M., ApJS 99, 713 (1995).

Saumon D., Hubbard W.B., Burrows A., Guillot T., Lunine J.I. \& Chabrier G., ApJ 460, 993 (1992).

Saumon D., Chabrier G., Wagner D.J., \& Xie X., High Pressure Research 16, 331 (2000a).

Saumon D., Geballe T.R., Leggett S.K., Marley M.S., Freedman R.S., Lodders K., Fegley B. \& Sengupta S.K., ApJ 541, 374 (2000b).

Schmider F.X., Mosser B. \& Fossat E., A $\mathcal{E} A$ 248, 281 (1991).

Seager S. \& Sasselov D.D., ApJ 502, L157 (1998).

Seager S. \& Sasselov D.D., ApJ 537, 916 (2000).

Seiff A., Kirk D.B., Knight T.C.D., Young R.E., Mihalov J.D., Young L.A., Milos F.S., Schubert G., Blanchard R.C \& Atkinson D., J. Geophys. Res. 103, 22857 (1998).

Showman A.P. \& Dowling T.E., Science 289, 1737 (2000).

Showman A.P. \& Guillot T., A\&A 385, 165 (2002).

Smoluchowski R., Nature 215, 691 (1967).

Stevenson D.J. Ph.D. Thesis, Cornell University (1976a)

Stevenson D.J., Phys. Lett. A 58, 282 (1976b).

Stevenson D.J. \& Salpeter E.E., ApJ Suppl. 35, 221 (1977a).

Stevenson D.J. \& Salpeter E.E., ApJ Suppl. 35, 239 (1977b).

Stevenson D.J., Ann. Rev. Earth Planet. Sci. 10, 257 (1982).

Stevenson D.J., Rep. Prog. Phys. 46, 555 (1983).

Stevenson D.J., Ann. Rev. Aston. Astrophys. 29, 163 (1991).

Strauss M.A. et al., ApJL 522, L61 (1999).

Sudarsky D., Burrows A. \& Pinto P., ApJ 538, 885 (2000).

Trilling D.E., Benz W., Guillot T., Lunine J.I., Hubbard W.B., \& Burrows A., ApJ 500, 428 (1998). 
Tsuji T., Ohnaka K., Aoki W. \& Nakajima T., A $\& A$ 308, L29 (1996).

Tyler G.L., Sweetnam D.N., Anderson J.D. et al., Science 246, 1466 (1989).

Vidal-Madjar A., Lecavelier des Etangs A.L., Désert J.-M., Ballester G.E., Ferlet R., Hébrard G.

\& Mayor M., Nature 422, 143 (2003).

Warwick J.W., Evans D.R., Roming J.H., et al., Science 223, 102 (1986).

Weidenschilling S.J., Astrophys. \& Sp. Sci. 51, 153 (1977).

Weir S.T, Mitchell A.C. \& Nellis W.J., Phys. Rev. Lett. 76, 1860 (1996).

Wigner E. \& Huntington H.B., Phys. Rev. B 3, 764 (1935).

Wuchterl G., Guillot T., \& Lissauer J.J. In Protostars 8 Planets IV, eds. V. Mannings et al., (Tucson: Univ. of Arizona Press), 1181 (2000)

von Zahn U., Hunten D.M., Lehmacher G., J. Geophys. Res. 103, 22815 (1998).

Zharkov V.N. \& Trubitsyn V.P., "Physics of Planetary Interiors", W.B. Hubbard ed., Pachart, Tucson (1978).

Zharkov V.N., "Interior structure of the Earth and planets", Harwood academic pubishers (1986).

Zharkov V.N. et Gudkova T.V., in "High pressure research: application to Earth and planetary sciences", (eds. Y. Syono et M.H. Manghnani), TERRAPUB, Tokyo (1992). 